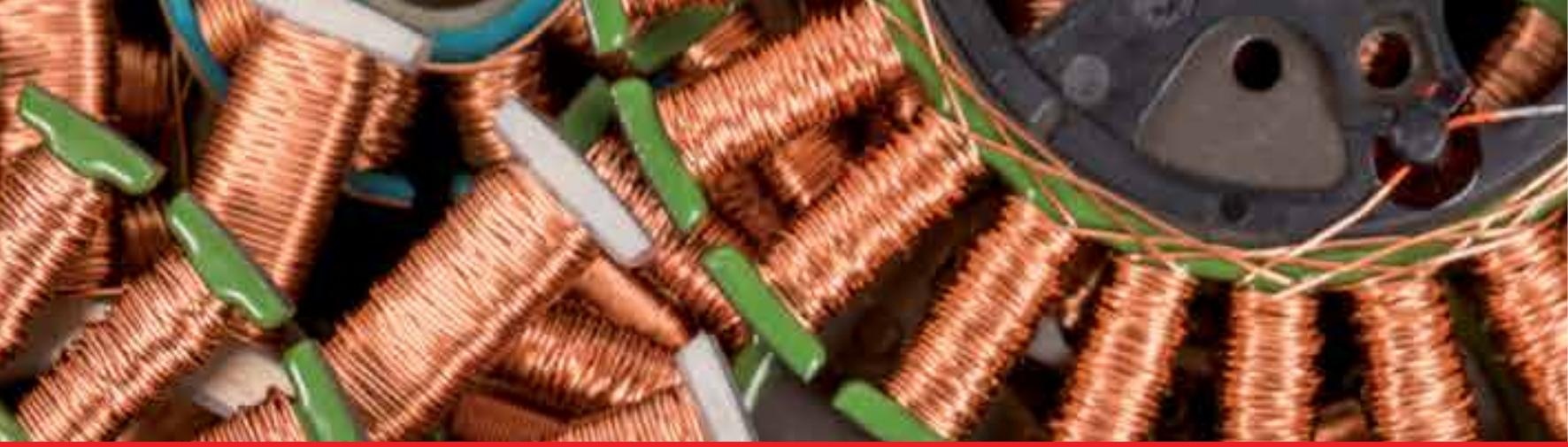

\title{
IntechOpen
}

\section{Applied Electromechanical Devices and Machines for Electric Mobility Solutions}

Edited by Adel El-Shahat and Mircea Ruba
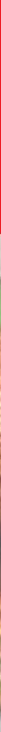



\section{Applied Electromechanical Devices and Machines for Electric Mobility Solutions}

Edited by Adel El-Shahat and Mircea Ruba 

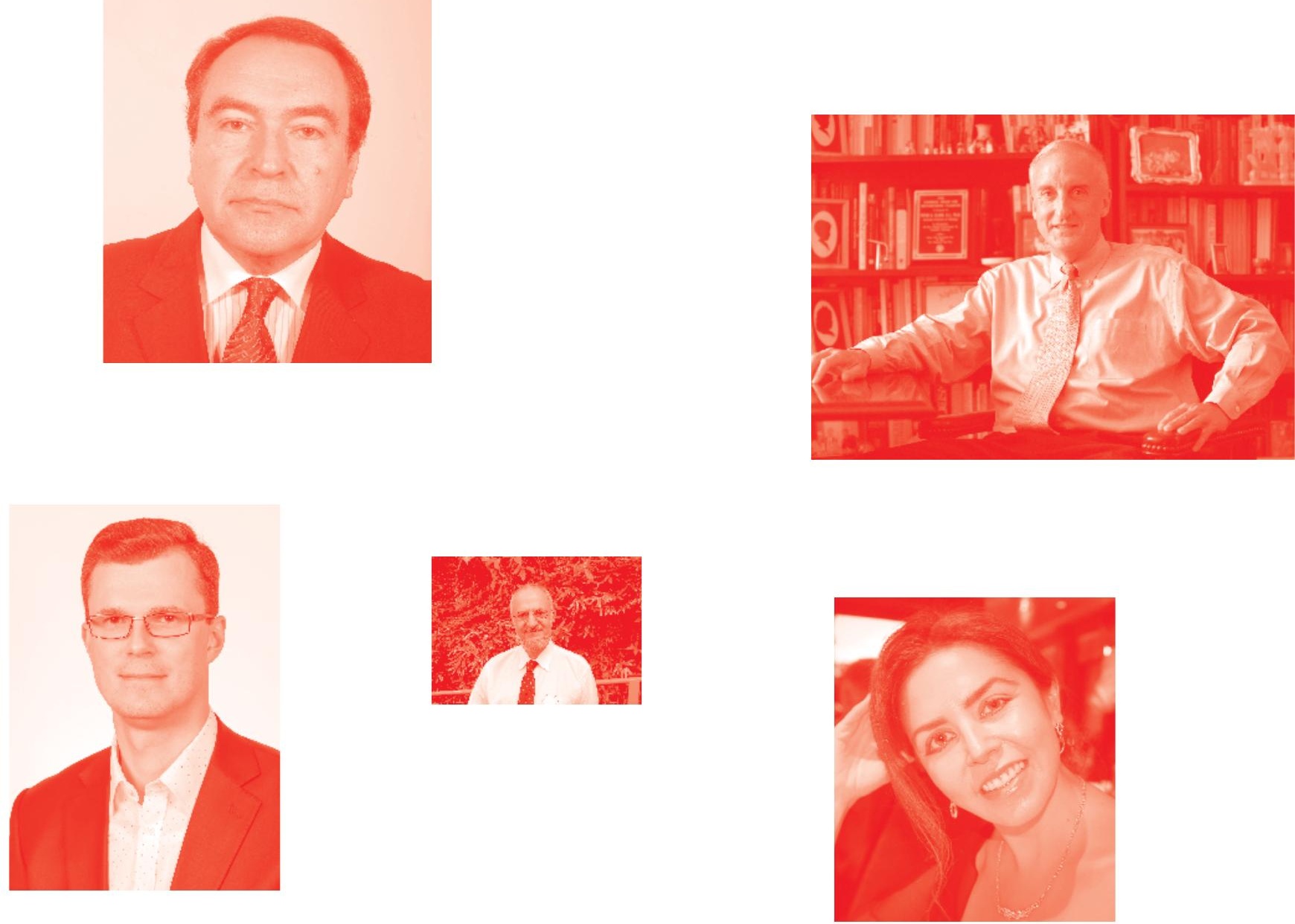

Supporting open minds since 2005
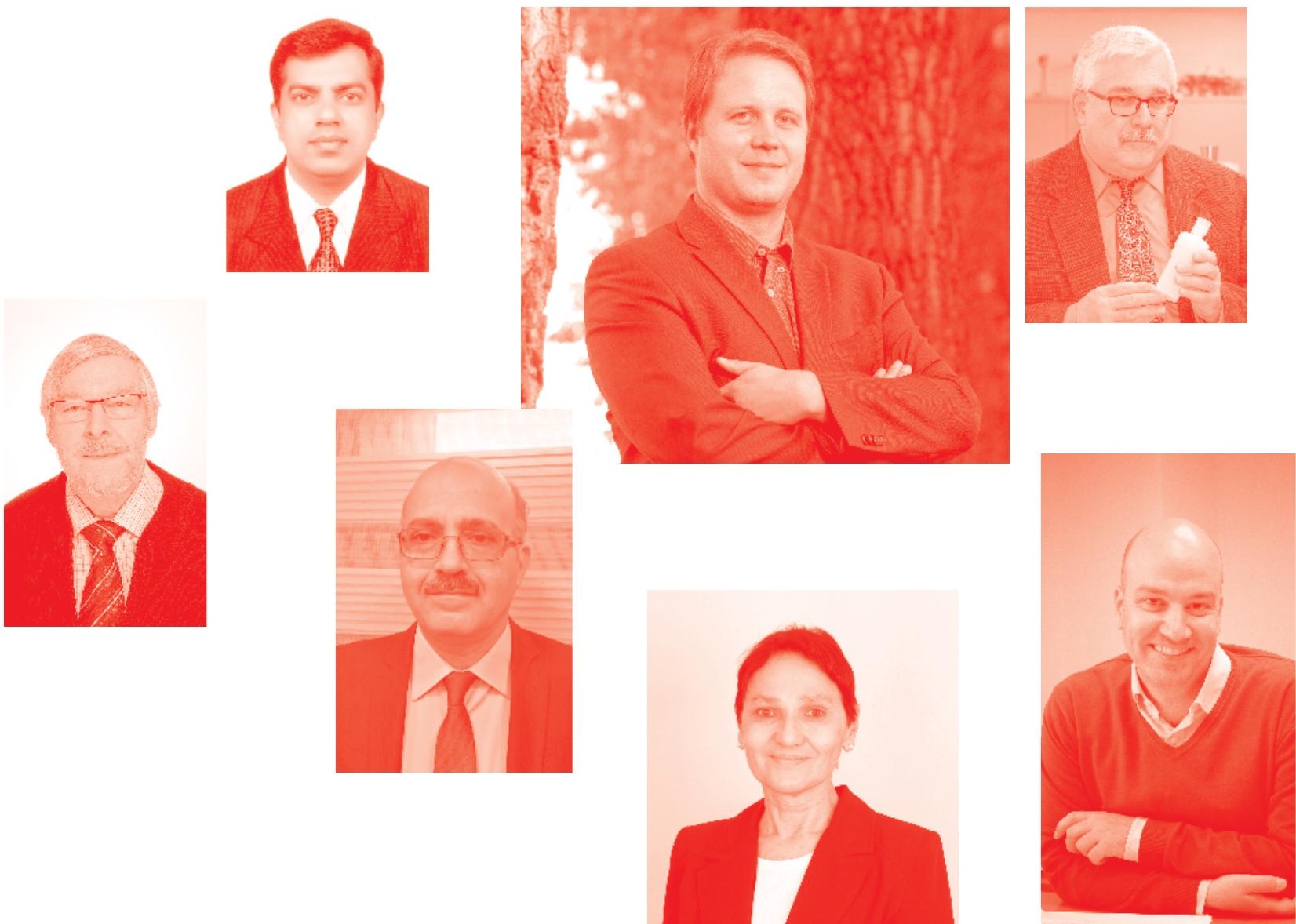
Applied Electromechanical Devices and Machines for Electric Mobility Solutions

http : //dx . doi.org/10.5772/intechopen. 83292

Edited by Adel El-Shahat and Mircea Ruba

\section{Contributors}

Mikhail Pustovetov, Chuan-Pei Lee, Po-Kang Yang, Dhara Patel, Leonimer Melo, Alex Archela, Dario Toginho, Khalid G Mohammed, Chang-Ming Liaw, Jia-Hsiang Zhuang, Shih-Wei Su, Kai-Wei Hu, Sorin Ioan Deaconu, Lucian Nicolae Tutelea, Nicolae Muntean, Feifei Bu, Marcel Topor, Adhavan Balashanmugham, Maheswaran Mockaisam, Hesham Rakha, Kyoungho Ahn, Enhua Wang, Xu Zhang, Yue Pan, Minggao Ouyang, Languang Lu, Xuebing Han, Guoqing Jin, Anjian Zhou, Huiqian Yang, Mircea Ruba, Raul-Octavian Nemes, Sorina-Maria Ciornei, Claudia Martis

( ) The Editor(s) and the Author(s) 2020

The rights of the editor(s) and the author(s) have been asserted in accordance with the Copyright, Designs and Patents Act 1988. All rights to the book as a whole are reserved by INTECHOPEN LIMITED . The book as a whole (compilation) cannot be reproduced, distributed or used for commercial or non-commercial purposes without INTECHOPEN LIMITED's written permission. Enquiries concerning the use of the book should be directed to INTECHOPEN LIMITED rights and permissions department (permissions@intechopen.com).

Violations are liable to prosecution under the governing Copyright Law .

\section{(cc) BY}

Individual chapters of this publication are distributed under the terms of the Creative Commons Attribution 3.๑ Unported License which permits commercial use, distribution and reproduction of the individual chapters, provided the original author(s) and source publication are appropriately acknowledged. If so indicated, certain images may not be included under the Creative Commons license. In such cases users will need to obtain permission from the license holder to reproduce the material. More details and guidelines concerning content reuse and adaptation can be found at http : //www . intechopen . com/copyright-policy . html .

\section{Notice}

Statements and opinions expressed in the chapters are these of the individual contributors and not necessarily those of the editors or publisher. No responsibility is accepted for the accuracy of information contained in the published chapters. The publisher assumes no responsibility for any damage or injury to persons or property arising out of the use of any materials, instructions, methods or ideas contained in the book.

First published in London, United Kingdom, 2020 by IntechOpen

IntechOpen is the global imprint of INTECHOPEN LIMITED, registered in England and Wales, registration number: 11086078 , 7th floor, 10 Lower Thames Street, London,

EC3R 6AF, United Kingdom

Printed in Croatia

British Library Cataloguing-in-Publication Data

A catalogue record for this book is available from the British Library

Additional hard and PDF copies can be obtained from orders@intechopen.com

Applied Electromechanical Devices and Machines for Electric Mobility Solutions

Edited by Adel El-Shahat and Mircea Ruba

p. $\mathrm{cm}$.

Print ISBN 978-1-78985-727-6

Online ISBN 978-1-78985-728-3

eBook (PDF) ISBN 978-1-83880-016-1 


\section{We are IntechOpen, \\ the world's leading publisher of Open Access books}

Built by scientists, for scientists

\section{$4,700+$}

Open access books available

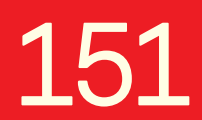

Countries delivered to

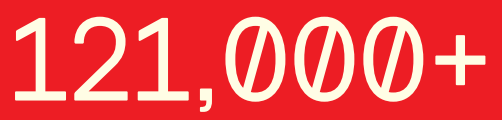

International authors and editors

Our authors are among the

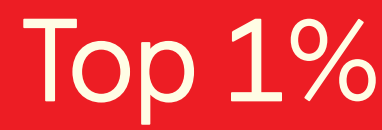

most cited scientists

Contributors from top 500 universities
40010

Downloads

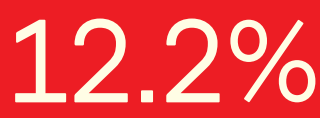

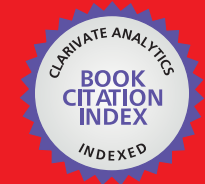

WEB OF SCIENCE ${ }^{\text {MM }}$

Selection of our books indexed in the Book Citation Index in Web of Science ${ }^{\mathrm{TM}}$ Core Collection (BKCI)

Interested in publishing with us?

Contact book.department@intechopen.com

Numbers displayed above are based on latest data collected.

For more information visit www.intechopen.com

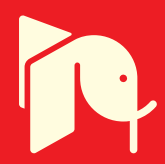





\section{Meet the editors}

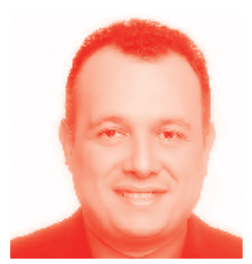

Dr Adel El-Shahat works in the Department of Electrical and Computer Engineering at Georgia Southern University, USA. $\mathrm{He}$ is a Founder and Director of Innovative Power Electronics and Nano-Grids Research Lab (IPENG). His research focuses on neural networks; electric machines; electric vehicles; smart grid systems; power electronics; power systems; energy storage and optimization. He has received several awards, recognitions, and honors due to his work. He is a Senior Member of IEEE, IRED, and a member of 22 professional organizations. He is Editor-in-Chief of the International Journal of Convergence Computing and a reviewer for 60 journals and conferences. He has authored 60 journal papers and 65 refereed conferences' papers plus 8 books and 5 book chapters with 100 other publications.

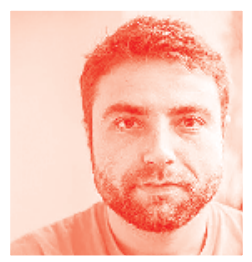

Dr Mircea Ruba is currently a lecturer with the Technical University of Cluj-Napoca (TUCN), Department of Electrical Machines and Drives and is a member of CAREESD, being in charge of the Real-Time System Modelling and Testing Laboratory (RTsym$\mathrm{Lab})$. His scientific interests are focused on electric vehicle (EV) propulsion drives and on their real-time FPGA based simulation and Hardware in the Loop (HiL) testing. He has been a researcher in over 20 projects and in most he worked to develop electrical motors, power inverters, controllers and battery supply units for EVs. His skills in designing, simulation and controlling propulsion motors for EVs have been proved in a large number of publications, creating real-time (RT) FPGA based simulators for their realistic analysis and control. Investigation of Lilon battery identification, simulation and testing was also a consistent research direction for Dr Ruba including patenting a novel, ultra-fast and efficient BMS. He invested several years and his $\mathrm{PhD}$ thesis in the study of fault tolerant machines, converters, and controllers outcome in many publications patenting a novel fault detection and compensation strategy and a modular fault tolerant motor design. His patents were awarded in 13 international exhibitions, his publications were cited in hundreds of papers, especially in 97 ISI journals and since 2018 he was invited to be an editor for "Devices and machines applied for electric mobility solutions" published by IntechOpen. He has published over 80 conference papers, 6 ISI journal papers, 4 books, and 6 patents ( 3 still pending). 



\section{Contents}

Chapter 1

Parameter Identification, Modeling and Testing of Li-Ion Batteries Used in Electric Vehicles

by Mircea Ruba, Raul Nemeș, Sorina Ciornei and Claudia Marțis,

Chapter 2

Permanent-Magnet Synchronous Machine Drives

by Adhavan Balashanmugham and Mockaisamy Maheswaran

Chapter 3

Investigation on Internal Short Circuit Identification of

Lithium-Ion Battery Based on Mean-Difference Model and

Recursive Least Square Algorithm

by Xu Zhang, Yue Pan, Enhua Wang, Minggao Ouyang, Languang Lu,

Xuebing Han, Guoqing Jin, Anjian Zhou and Huiqian Yang

Chapter 4

Induction Electrical Machine Simulation at Three-Phase Stator

Reference Frame: Approach and Results

by Mikhail Pustovetov

Chapter 5

CMOS Active Inductor and Its Applications

by Dhara Pinkesh Patel

Chapter 6

Low-Cost (PM Less) Wide-Speed-Range-Operation Generators by Sorin Ioan Deaconu, Feifei Bu, Marcel Topor,

Lucian Nicolae Tutelea and Nicolae Muntean

Chapter 7

A Simple Hybrid Electric Vehicle Fuel Consumption Model for Transportation Applications

by Kyoungho Ahn and Hesham A. Rakha

Chapter 8

Driving Control Technologies of New High-Efficient Motors by Chang-Ming Liaw, Jia-Hsiang Zhuang, Shih-Wei Su, Kai-Wei Hu and G. Vijay Kumar 
Chapter 9

Mechanical and Electrical Design Calculations of Hybrid Vehicles by Khalid G. Mohammed

Chapter 10

Torque Control of a DC Motor with a State Space Estimator and Kalman Filter Applied in Electrical Vehicles by Alex Archela, Dario Guilherme Toginho and Leonimer Flávio de Melo

Chapter 11

2D-Layered Nanomaterials for Energy Harvesting and Sensing Applications by Po-Kang Yang and Chuan-Pei Lee 


\section{Preface}

This book addresses recent advances and multidisciplinary advanced topics of applied electromechanical devices and machines for electric mobility solutions. The presented research will help to shape the future of this important area and help researchers to solve many problems and challenges. This book is a unique introduction to applied electromechanical devices and machines for electric mobility solutions. It examines the current state and future in this field and unpacks the complicated relationships between its theoretical, experimental, and technical variables. It supplies the reader with an integrated view of the latest research to support graduates, senior-level undergraduate students, professionals, and researchers to learn basic and advanced topics of the proposed techniques. It presents both theoretical knowledge and hands-on work from experts.

It does so by addressing advanced applications and innovative case studies for electromechanical parameter identification, modeling, and testing of; permanentmagnet synchronous machine drives; investigation on internal short circuit identifications; induction machine simulation; CMOS active inductor applications; low-cost wide-speed operation generators; hybrid electric vehicle fuel consumption; control technologies for high-efficient applications; mechanical and electrical design calculations; torque control of a DC motor with a state-space estimation; and 2D-layered nanomaterials for energy harvesting. This book is essential reading for students, researchers, and professionals interested in applied electromechanical devices and machines for electric mobility solutions.

The proposed book topics are well prepared and presented in the form of eleven chapters as follows:

Chapter (1): Parameter Identification, Modelling, and Testing

Chapter (2): Permanent-Magnet Synchronous Machine Drives

Chapter (3): Investigation on Internal Short Circuit Identification of Lithium-Ion Battery

Chapter (4): Induction Electrical Machines Simulation at 3-phases Reference-Frame

Chapter (5): CMOS Active Inductor and its Applications

Chapter (6): Low Cost Wide-Speed-Range Operation Generators

Chapter (7): A Simple Hybrid Electric Vehicle Fuel Consumption Model for Transportation Applications

Chapter (8): Driving Control Technologies

Chapter (9): Mechanical and Electrical Design Calculations of Hybrid Vehicles 
Chapter (10): Torque Control of a DC Motor with a State Space Estimator and Kalman-Filter

Chapter (11): 2D-Layered Nanomaterials for Energy Harvesting and Sensing Applications

\section{Dr. Adel El-Shahat}

Senior IEEE Member,

Assistant Professor,

Department of Electrical and Computer Engineering, Founder \& Director of Innovative Power Electronics \&

Nano-Grids Research Lab. (IPENG),

Georgia Southern University, Statesboro, Georgia, USA

Dr. Mircea Ruba

Lecturer,

Electrical Engineering Faculty,

Technical University of Cluj-Napoca,

Romania 


\title{
Parameter Identification, Modeling and Testing of Li-Ion Batteries Used in Electric Vehicles
}

\author{
Mircea Ruba, Raul Nemeș, Sorina Ciornei and Claudia Marțis
}

\begin{abstract}
The chapter focuses on presenting a detailed step-by-step workflow for theoretical and practical approach of Li-ion battery electric parameter identification. Correct and precise information about the electric parameters of the batteries allows defining several types of simulation approaches. Increasing the complexity of these approaches requires more and more identified parameters and by this more complicated hardware to fulfill the identification process itself. However, the level of complexity must be justified by the need of accuracy as well as the compromise of labor, simulation power and time. In this chapter, several simulation complexity levels are presented via theory and then tested via simulations compared with actual measurements. A proper and well-done analysis of these models helps the future reader to decide whether he will use a complex model, function of the need of accuracy, simulation power and time. The compromise will be highlighted by comparing the error of different approaches compared to actual laboratory measurements. Over all, the chapter will be a gathered guideline for identification, modeling and testing of batteries, ready to be implemented both in simulation and in practical experiments.
\end{abstract}

Keywords: battery, parameter identification, simulation and modeling, model complexity benchmark

\section{Introduction}

Nowadays, the interest of research in the field of batteries, both from the electrical and chemical perspectives, gained a lot of field. Many R\&Ds from both industry and academia are engaged in developing design, simulation, hardware testing and performance analysis solutions to better the existing batteries. Electrification of transport, both in the area of heavy-duty and light solutions, requires higher and higher performances of the batteries. This influences directly the autonomy of the vehicle reflecting directly the owner's comfort and trust in investing in such new vehicles. The research invested in batteries for increasing more power density into each cell comes with a lot of effort, it is time consuming and returns in higher costs. Nevertheless, thermal stability of the batteries is a very delicate issue, knowing unfortunate events that occurred when batteries caused fires and human injury as well. 
The evolution of technology both in engineering and software, reduced the time to market and proper evolution of the batteries, increasing performances whilst diminishing the development costs. Solutions to study batteries are included in a large variety of software; models are already preprogramed into hardware power emulators, ready for use. However, those are often closed-source tools with access only to replace the battery parameters, many times with linear ones. It was proven in many studies that the main electrical parameters of the batteries are far from being linear. Even more, it is known that aging, cell temperature and ambient temperature are extremely aggressive in changing the battery parameters.

Hence, many times custom-made battery electric models are more than a good solution to run studies where the designer can add or dismiss many factors and parameters that are varying with several external influences. In the same time, designing such models simplifies the transition from off-line analysis to real-time applications. The latter tool is not always at the disposal of the designer due to platforms that are dedicated exclusively to computer-based simulators.

On the other hand, performing proper parameter identification of existing cell, to validate their theoretical design, can be quite challenging. There are many published papers that reflect these methods that some are quite simple and lucrative solutions, while others are complex and require expensive setup and a lot of data post-processing.

The present chapter will engage this issue of parameter identification for Li-ion battery cells and present the main used simulation models, and benchmarking of these models will give the reader a certain definition of the advantages and drawbacks of some models versus others. The chapter does not include information regarding the battery control strategies or battery management topics, remaining focused only on the above-mentioned subjects to be presented in detail. Also parameter variations due to large temperature variations or other stress factors were not taken into discussion as these variations can be recorded using the presented methods while imposing such external factors to the subjected battery cells.

In the authors' opinion, there are two main directions of judging the complexity of a model. If considering industrial work, engaging models with lower complexity and good accuracy, is a lucrative solution to reach the desired target not forgetting to decrease the time to market. On the other hand, in academia-based research, more complex models with very high accuracy is the key to prove the designer's skill, to prove the model's benefit and to push to reach a used solution on the large scale. The latter comes with the drawback of high complexity that demands large time consumption and a lot of involved labor.

In the present chapter, such a comparison will prove the above-mentioned aspects while comparing two types of different simulation models for the same type of battery.

\section{Battery analysis models}

As the batteries are electrochemical entities, there are several directions when approaching and building a simulation model to perform its behavioral analysis. The model can be designed from the chemical or electrical point of view or can be a hybrid mixture between them. Moreover, the battery temperature, as critical parameter, is often analyzed using a thermal model as add-on to the above-mentioned ones.

In the literature, an electrochemical approach is the pseudo-two-dimensional model developed by Doyle [1], which proved to be able to predict quite well the 
dynamics of Li-ion batteries. The main disadvantage of such a model is the high computational required time.

The authors will approach only the electrical simulation models for batteries due to their electrical engineering education. However, research on batteries should always be considered as a multidisciplinary domain, gathering researchers educated in chemical, electrical and thermal sciences in order to be able to perform a complete and realistic battery cell model.

In the literature, there are several approaches for the electrical simulation of battery cells [2-6]; mainly all are based on electric circuit models (ECM). Function of the battery chemistry, of the required model accuracy and of the designer, there are available simple circuits such as the Thevenin approach [7], complex ones, such as the impedance based spectroscopy approach [8] and middle range circuits such as the first and second order electric circuits [9-12]. The above-mentioned categories range their accuracy from satisfactory to highly precise.

The present chapter will approach the first- and second-order electric circuitbased modeling, detailing aspects such as model design, parameter identification and accuracy testing.

When using the expression that classifies these models into first and second order, these are referred to the number of parallel resistance-capacitance groups used to describe the battery.

In Figure 1 (left and right), the generic electric circuits for the first- and secondorder models, respectively, are depicted. Both have the first resistance in common
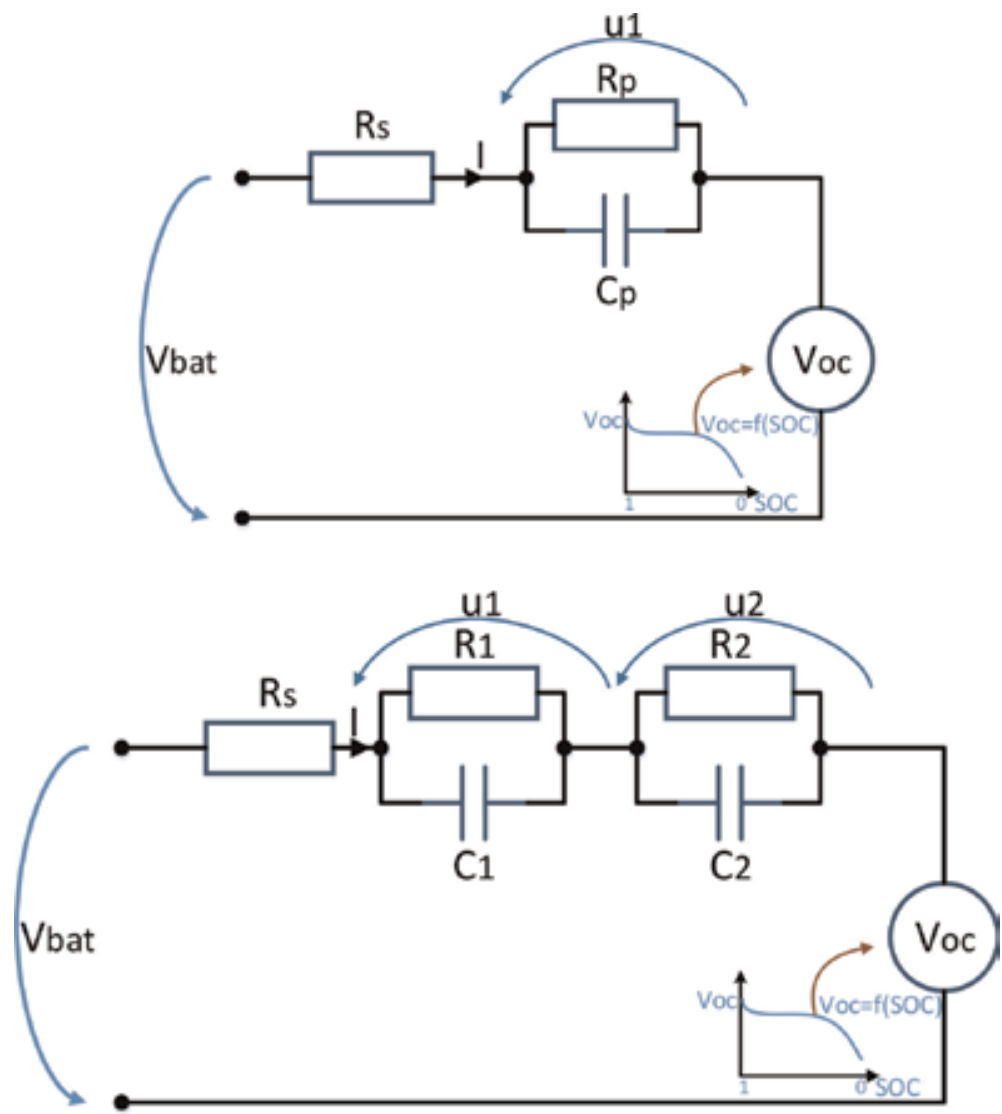

Figure 1.

The ECM for the first-order (top) and second-order (bottom) approaches. 
$\left(R_{s}\right)$, this being referred to as the internal series resistor that is responsible with the ohmic considered resistance of the battery cell. In Figure 1 (left), in the first-order approach, the $\mathrm{RC}$ parallel group has the role of replicating the dynamic transient of the voltage inside the cell. Its components stand for the polarization resistance $\left(R_{p}\right)$ and the polarization capacitance $\left(C_{p}\right)$. The internal voltage source, denoted with $\left(\mathrm{V}_{\mathrm{oc}}\right)$, represents the open circuit voltage of the Li-ion cell function of its state of charge (SOC).

Now in order to model the first-order ECM, first one has to apply Kirchhoff's law on the circuit, composed of tree voltage drops: the open circuit one, the RC parallel group and the series resistance one, explained in Eq. (1).

$$
V_{b a t}=V_{o c}-u_{1}-R_{s} \cdot I_{b a t}
$$

In Eq. (1), the current drained from the battery (or supplied to it, in case of charging) is denoted with ( $\left.\mathrm{I}_{\mathrm{bat}}\right)$. The voltage drop of the parallel RC connection is simple to be expressed like a derivative:

$$
\frac{d u_{1}}{d t}=-\frac{1}{R_{p} \cdot C_{p}} u_{1}+\frac{1}{C_{p}} \cdot I_{b a t}
$$

The open circuit voltage $\left(\mathrm{V}_{\mathrm{oc}}\right)$ represented function of the SOC of the cell is in fact a recorder data from actual cells; however, details about that will be explained later on in the following pages.

Comparing the two circuits depicted in Figure 1, one can conclude that in fact, the second-order model includes the first-order one having in addition a second RC parallel group. However, the concept is not straightforward. In the first-order model, the entire polarization dynamics are handled using one RC group. For the second-order model, this dynamical process is replicated using two such RC groups as it can be seen in Figure 1. The series resistance and the open circuit voltage components are the same as for the first-order model, while the $\left(\mathrm{R}_{1} \mathrm{C}_{1}\right)$ and $\left(\mathrm{R}_{2} \mathrm{C}_{2}\right)$ groups denote the activation polarization and the concentration polarization, respectively.

Hence, based on this circuit, the second-order model based again on Kirchhoff's law can be described using Eq. (3).

$$
\begin{aligned}
& V_{b a t}=V_{o c}-u_{1}-u_{2}-R_{s} \cdot I_{b a t} \\
& \frac{d u_{1}}{d t}=-\frac{1}{R_{1} \cdot C_{1}} u_{1}+\frac{1}{C_{1}} \cdot I_{b a t} \\
& \frac{d u_{2}}{d t}=-\frac{1}{R_{2} \cdot C_{2}} u_{1}+\frac{1}{C_{2}} \cdot I_{b a t}
\end{aligned}
$$

The analytical approach for the first- and second-order models described in Eqs. (1)-(3) are exposed as pure electric equations. However, it is known that the parameters used in this model, such as resistance, capacitances, open circuit voltage, or state of charge, must be identified from actual battery cells. Hence, in order to create a link between the analytical battery models and the identification process, both for the first- and second-order models, another approach can be engaged. This drives more forward the mathematical perspective of modeling dynamic signal variations. Using the exponential mathematical function, the models from the above-mentioned equations can be reorganized as follows:

- For the first-order model,

$$
V_{b a t}=k_{0}-k_{1} \cdot \exp (-a \cdot t)
$$


where the terms $\left(k_{0}\right),\left(k_{1}\right)$ and (a) can be identified from the above equations as coefficients

$$
\begin{aligned}
& k_{0}=V_{o c}-R_{s} \cdot I_{b a t} \\
& k_{1}=\frac{R_{1}}{I_{b a t}} \quad a=\frac{1}{R_{1} \cdot C_{1}}
\end{aligned}
$$

- For the second-order model

$$
V_{b a t}=k_{0}-k_{1} \cdot \exp (-a \cdot t)-k_{2} \cdot \exp (-b \cdot t)
$$

where again by the same identification result the coefficients

$$
\begin{array}{ll}
k_{0}=V_{o c}-R_{s} \cdot I_{b a t} & k_{2}=\frac{R_{2}}{I_{b a t}} \\
k_{1}=\frac{R_{1}}{I_{b a t}} & b=\frac{1}{R_{2} \cdot C_{2}}
\end{array}
$$

It is important to understand that using this second approach for both the models, one can simply link the battery characteristics to its parameters in order to be able to describe the complete model judging the correct interpretation of the measured values during the parameter identification process.

\section{Battery parameter identification}

The process of identifying the parameters that are then able to cope with the analytical model to describe the cell's behavior requires a preliminary hardware setup dedicated for such applications. There are several possibilities to build such a test bench. For the present study, the authors built a test bench based on a programmable electronic load and a programmable electronic supply, as depicted in Figure 2.

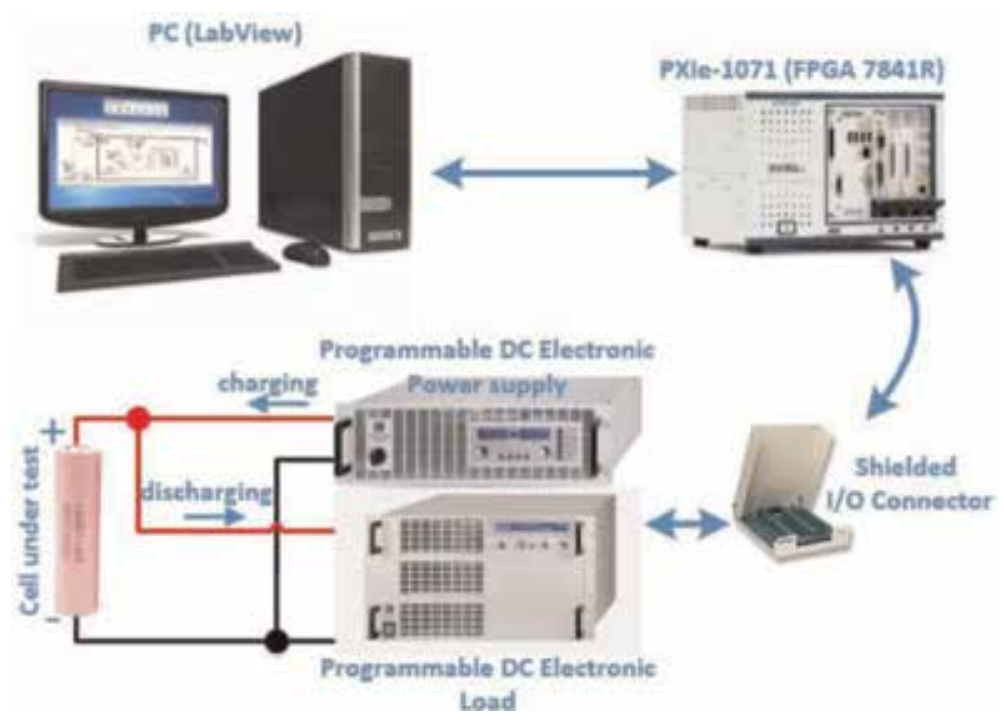

Figure 2.

The test bench for battery parameter identification process and testing. 
The used battery to model is a LG (LGABD11865) battery with a rated capacity of $3000 \mathrm{mAh}, 3.75 \mathrm{~V}$ rated, $4.2 \mathrm{~V}$ maximum over charge voltage, $2.7 \mathrm{~V}$ minimum discharge voltage, 0.5-1 A charging current and 0.2-0.5 A discharging current.

For the identification process, the battery was connected to a programmable load (EA-EL 9400-150 0-400 V 0-150A $7200 \mathrm{~W}$ ). From a host computer, the battery was discharged at $1 \mathrm{C}$ from $100 \%$ state of charge (SOC) till it reached the cut-off voltage. The flowchart of the identification process is depicted in Figure 3. Preliminarily, the battery is charged to $100 \%$ SOC using a commercial charger. It is left then to relax for $24 \mathrm{~h}$. Afterwards, it is connected to the programmable electronic load that is controlled by the host computer, used also to stream the $\mathrm{V}_{\mathrm{bat}}$ and $\mathrm{I}_{\mathrm{bat}}$ to data files, all versus the elapsed time.

The process starts by applying a 1C negative current pulse for a period of $60 \mathrm{~s}$, by this starting the decay of the battery voltage as it is discharged. After the $60 \mathrm{~s}$ pulse, the cell is left in relaxation for $180 \mathrm{~s}$. Before performing a new current pulse of $60 \mathrm{~s}$, the battery voltage is compared to the lowest threshold $\left(\mathrm{V}_{\mathrm{trs}}\right)$ of $2.7 \mathrm{~V}$. If the voltage is larger than $2.7 \mathrm{~V}$, the pulse is applied for another $60 \mathrm{~s}$ followed by $180 \mathrm{~s}$ relaxation period. If the voltage is less than $2.7 \mathrm{~V}$, the process stops as the battery is considered fully discharged.

Through the entire process, while $V_{\text {bat }}$ is larger than $V_{\text {trs }}$, the data is recorded and streamed to external files, with a sampling of 10 sample/s.

The resulted variation of the voltage function of the discharge current is depicted in Figure 4, where the $60 \mathrm{~s}$ length pulses of $-3 \mathrm{~A}$ were followed by a relaxation period of $180 \mathrm{~s}$. With such recorded data, one can proceed with the identification process for both first- and second-order model parameters.

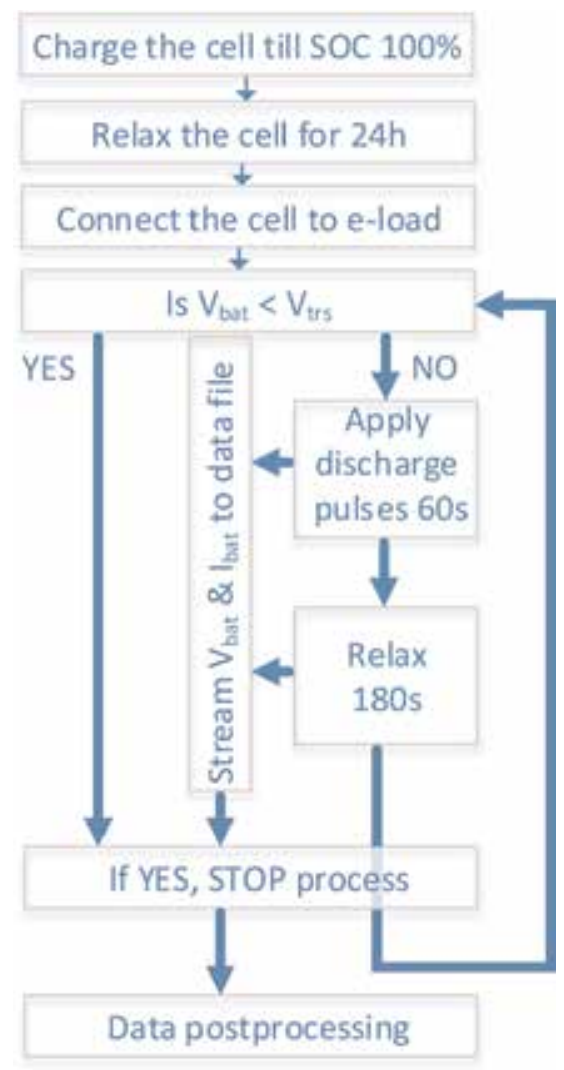

Figure 3.

The flowchart for the battery identification process data recording. 

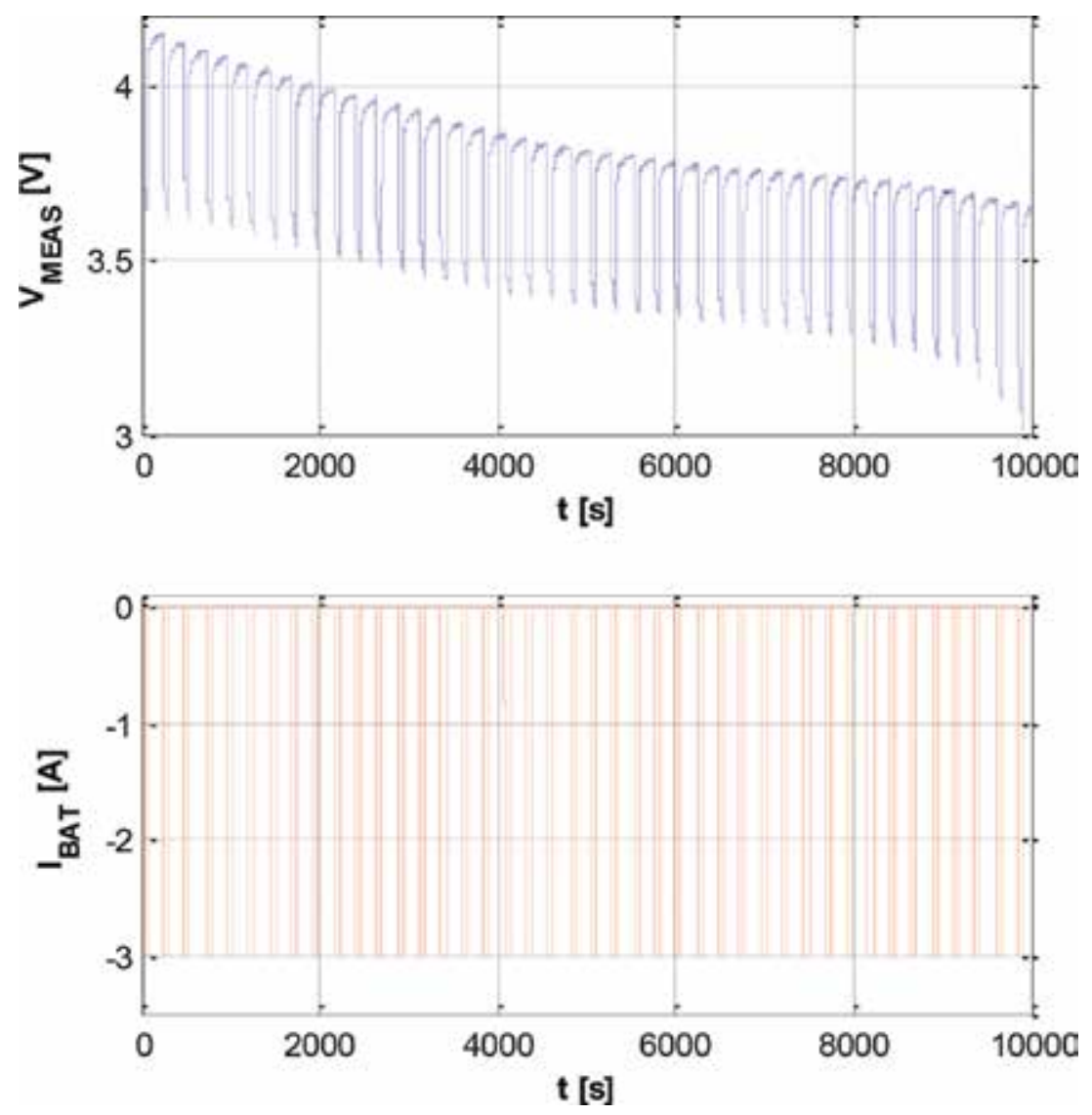

Figure 4.

The battery voltage (top) and the discharge current (bottom).

The data recorded into the external files is afterwards processed for the actual parameter identification of the Li-ion cell. Section 3.1 details all the necessary information for a clear hands-on methodology of data post-processing for parameter identification.

\subsection{Open circuit voltage and series resistance identification}

Independent for what type of circuit the designer wishes to build, first or second order, there are some parameters that are mandatory to be identified for both approaches. These parameters are the open circuit voltage $\left(\mathrm{V}_{\mathrm{oc}}\right)$ and the series resistance $\left(R_{s}\right)$. To understand their identification and role in the battery behavior, from Figure 4, the period according to one discharge current and one relaxation time is zoomed in Figure 4. All the identification steps will be explained with regard to this figure.

In Figure 4 it can be seen that $\left(\mathrm{V}_{\mathrm{oc}}\right)$, corresponding to each pulse, is in fact the open circuit voltage measured just prior to the current pulse that will discharge the battery. This value is measured from SOC $100 \%$ till $10 \%$ and recorded as $V_{\text {oc }}=f$ (SOC), as depicted in Figure 6.

The second parameter, the series resistance $\left(R_{s}\right)$ that creates the large visible voltage drop when applying the discharge current, can be quite easily computed. Knowing the $\mathrm{V}_{\mathrm{oc}}$ at the start of each discharge pulse and measuring the $\left(\mathrm{V}_{2}\right)$ voltage 


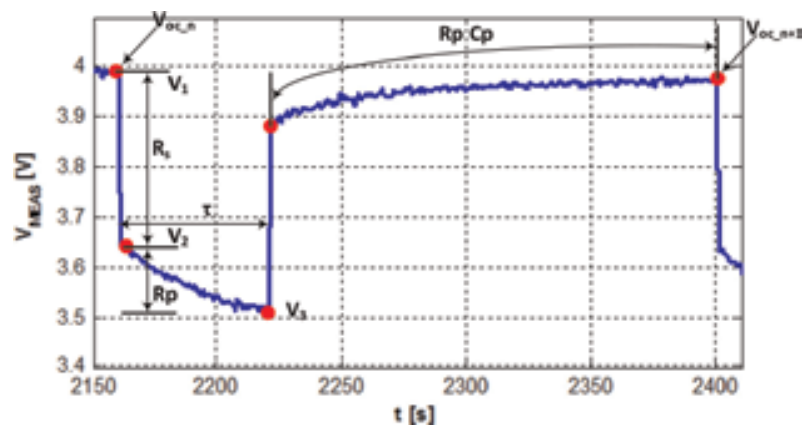

Figure 5 .

The detailed voltage variation for the battery parameter identification (first-order model).

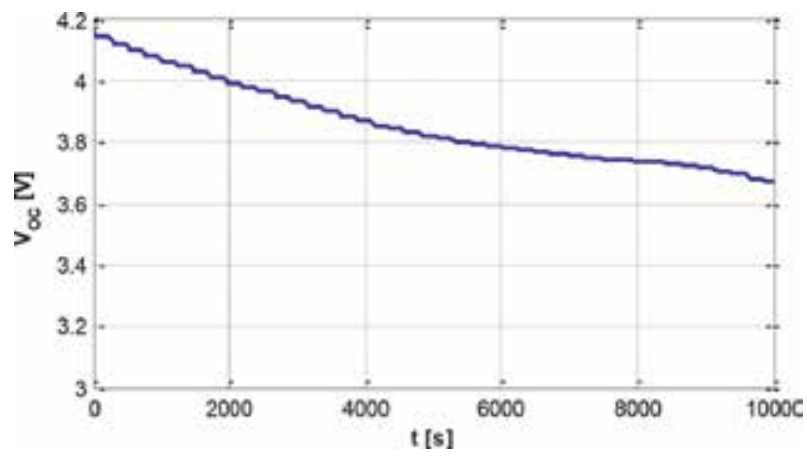

Figure 6.

The open circuit voltage for the entire discharge cycle.

(from Figure 5) and knowing the value as well of the pulsed current applied make it easy to compute the $\left(\mathrm{R}_{\mathrm{s}}\right)$ resistance based on Ohm's law (see Eq. (8)):

$$
R_{s}=\frac{V_{o c}-V_{1}}{I_{b a t}}
$$

From this point on, the post-processing of the acquired data to identify the parameters for the first- and second-order models will by slightly different. To be able to clearly understand each process, these will be treated separately with both equations and explanations, coped with the represented parameters from Figure 5.

\subsubsection{First-order parameter identification}

Going back to Eqs. (1) and (2), one can see that the open circuit voltage together with the series resistances was already identified as a general parameter for all battery models. What remains to be calculated are the polarization resistance $\left(R_{p}\right)$ and polarization capacitance $\left(\mathrm{C}_{\mathrm{p}}\right)$. In Figure 5, it can be seen that for each discharge current pulse, the lowest value of the voltage is marked with $\left(V_{3}\right)$. Based on Ohm's law, coped with the discharge current, one can simply compute the value of the polarization resistance as in Eq. (9).

$$
R_{p}=\frac{V_{2}-V_{3}}{I_{b a t}}
$$

On the other hand, the value of the $\left(\mathrm{V}_{3}\right)$ voltage function of time is influenced by the polarization capacitance of the battery $\left(C_{p}\right)$. Therefore, knowing the value of 
the polarization resistance and the time length of the current pulse $(\tau)$, it is straightforward to compute the capacitance as in Eq. (10).

$$
\tau=R_{p} \cdot C_{p}
$$

Generally, these computations are done during the discharging periods; however, these are valid to be computed for the relaxation time as well. Normally, the voltage variation gradient both in discharge and relaxation periods should be the same, and this justifies the previous explanation. As the entire data recorder when proceeding with the battery identification process is a function of the SOC, the calculated parameters will be as well a plotted function of the SOC. In Figure 6, for the first-order model, the open circuit voltage, the series and polarization resistances and the polarization capacitance for the entire range of battery discharge are plotted. It can be observed that the series resistance has a generally constant value over the entire period, while the polarization one increases when the SOC is over $90 \%$. In the same manner, the polarization capacitance as 3 regions, one between 0 and $40 \%$ SOC where is it quite constant, then between $40 \%$ and $80 \%$, a region with larger values and decreases a lot when the cell's SOC goes for the fully charged value.

\subsubsection{Second-order parameter identification}

In order to quantify the parameters for the second order, the same zoomed plot from Figure 5 is used again, but this time, the approach is slightly different. In Figure 7, the parameters to be identified are detailed in the voltage variation. The open circuit voltage and the series resistance quantification remains the same as detailed in the first paragraphs of Section 3.1. The remaining parameters to be calculated are linked to the model expressed in Eqs. (6) and (7). The reason of using exponential expressions instead of derivative ones is justified by the fact that the two RC groups of the second-order ECM are difficult to be separated. Hence, the shape of the voltage during the relaxation time (or the discharge time) is directly described by these two RC groups connected in series.

Actually, in the exponential voltage variation, one can observe that visually there is a period of fast variation and then a second one, of slow variation. It is impossible to define clearly these periods and to admit that one group is responsible for one period and the other one for the second period. Hence, the identification process includes both groups altogether. The reason of describing the battery model with exponential variations is justified in fact by the method of calculating these four parameters $\left(R_{1}, R_{2}, C_{1}\right.$ and $\left.C_{2}\right)$. This method is handled using a curve fitting procedure, based on the measured voltage shape. Using the fit function programmed in MATLAB Coder and applied for each relaxation period over the entire discharge cycle of the battery will return preliminary values for the four parameters, corresponding to each period. In order to give the reader a clear stepby-step procedure of handling this identification process, the authors considered that it is more lucrative to detail this post-processing phase of the identification:

- First, record all the transient regimes for each relaxation time into a matrix.

- Apply the curve fitting procedure for each of the recorded variations based on Eq. (6).

- Save the resulting constants $(\mathrm{k} 0, \mathrm{k} 1, \mathrm{k} 2, \mathrm{a}, \mathrm{b})$ into another matrix.

- Compute for each set of constants the values for $R_{1}, R_{2}, C_{1}$ and $C_{2}$. 

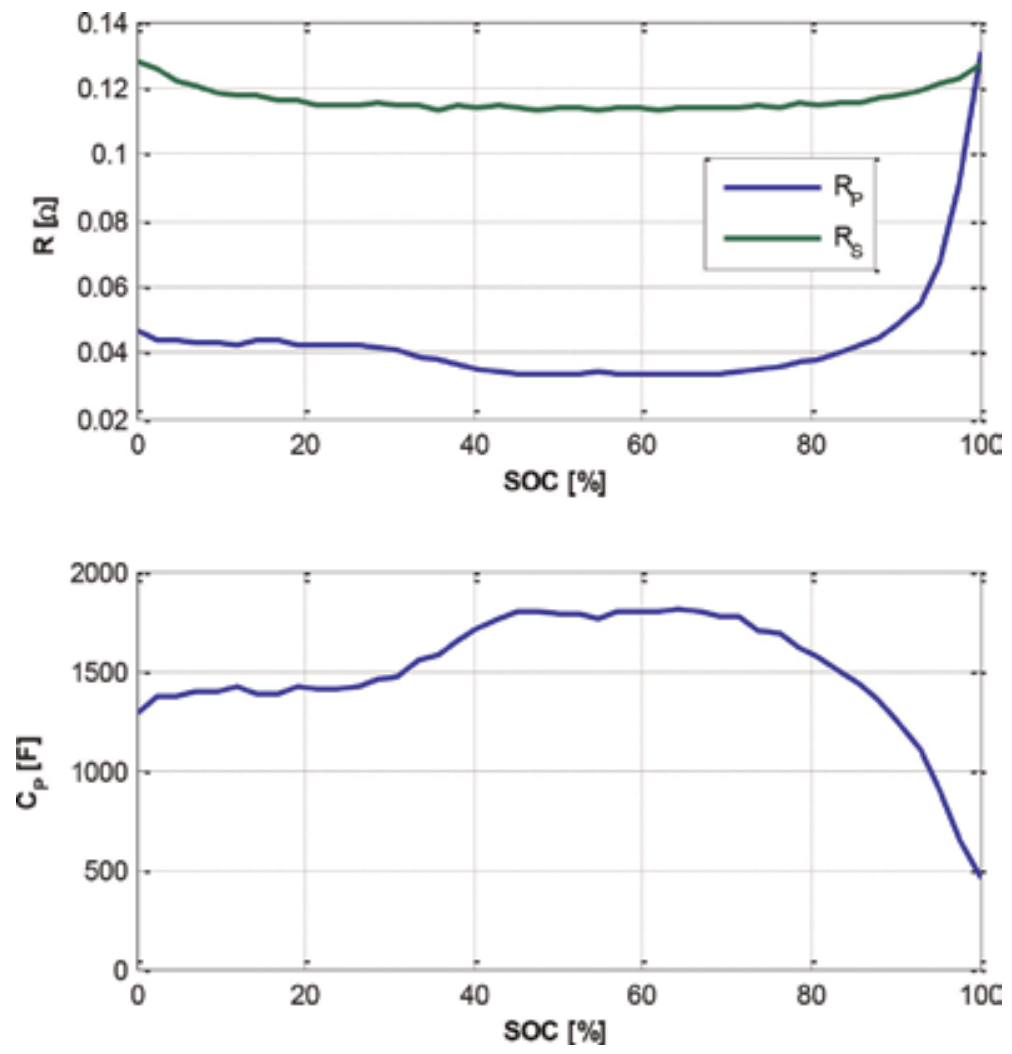

Figure 7.

The detailed voltage variation for the battery parameter identification (second-order model).

As general settings for the fit function, the best approach is to use nonlinear least squares, bounded by upper and lower values that are mandatory to be positive and realistic. The results of the fitting process will return the battery parameters that fit quite well with the measured voltage variation when reconstructing it based on the computed data. However, higher accuracy can be reached if one applies an optimization procedure of the fitted parameters to the actual measured voltage shape.

In Figure 8 (top), the actual measured voltage $\left(\mathrm{V}_{\mathrm{MEAS}}\right)$ with blue and the voltage variation obtained using Eq. (6) with the fitted parameters $\left(\mathrm{V}_{\text {FITT }}\right)$ with black are depicted. It is noticeable that there is some difference between the two characteristics, as well as in Figure 8 (bottom), with black; the difference between the two is highlighted in black, following the variation of $\left(\operatorname{err}_{\text {FITT }}\right)$.

On the other hand, if one applies an optimizer to fit more accurately with the battery parameters, the results reveal a highly precise characteristic that superimposes quite well on the measured one. In Figure 8 (top), with red, the voltage variation using optimized parameters is plotted $\left(\mathrm{V}_{\mathrm{OPT}}\right)$, while in Figure 8 (bottom), the ( $\operatorname{err}_{\mathrm{OPT}}$ ) is much smaller than the one obtained without optimization.

For the optimization process, the authors used a tool offered by MATLAB Simulink, called Control and Estimation Tool Manager. The benefit of using the tool provided by MATLAB Simulink is that the user does not have to have mathematical skills to implement optimization algorithms, investing his time and effort directly in using those existing for optimizing his model in development. This tool uses the model of the battery designed in MATLAB Simulink with the fitted parameters and varies them until the smallest error between the measured voltage variation and the model output is reached. This optimization is carried out for each relaxation time, as 
it was done for the preliminary fitting process. The resulted data are recorded into a matrix and will result in the battery parameter function of the SOC of the battery.

In Figure 9 (top left), the main window of the Control and Estimation Tool Manager is depicted. There are several already implemented optimization algorithms that can be chosen, setting the number of iterations, the tolerance and the search method for each of them. One example is highlighted in Figure 9 (top right) that shows the variations of the parameters carried out by the optimizer to reach the

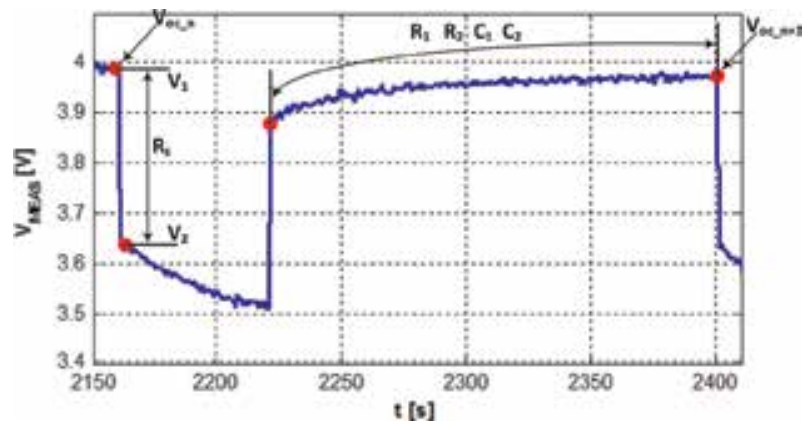

Figure 8.

Comparison of the fitted and optimized parameters influence (second-order model).
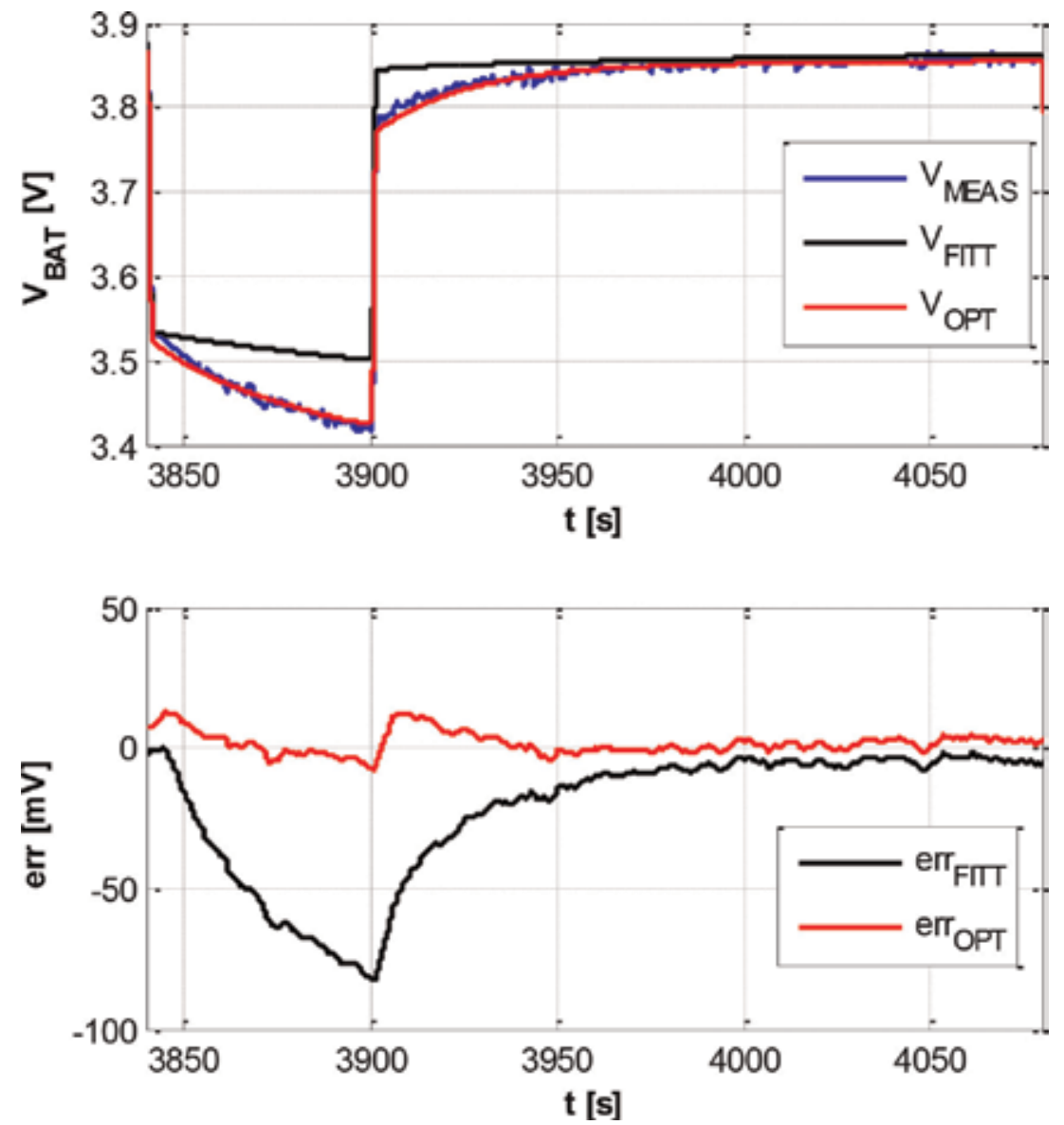

Figure 9.

The MATLAB Simulink Control and Estimation Tool Manager. 
closest battery model output voltage variation compared to the measured one. The final comparative result is depicted in Figure 9 (bottom), proving a closely complete overlap between the measured and the simulated curves, obtained for the new optimized battery parameters.

In Figure 8-bottom, comparing the results of the fitted and optimized models it is clear that in case of the latter, only $10 \mathrm{mV}$ of error is reached while the fitted one yells for maximum of $70 \mathrm{mV}$ error. Indeed, the labor and computation time for obtaining the optimized parameters is larger; however, if one requires high precision, it is the compromise that needs to be considered.

\section{Battery model benchmarking}

In the previous chapters, first- and second-order battery models with their equivalent circuits, parameter identification process and post-processing computations were presented. It is logical that the first-order battery model is the simplest one, yelling for simple parameter identification, simple modeling and simple data post-processing. However, undoubtable the accuracy of the first-order model is comparatively lower than the accuracy of the second-order model, especially by its nature that lacks components to describe the exponential transient of the battery voltage. It's been proved however [10] that such first-order models can be used to create fast, reliable and realistic simulation programs. In order to increase the level of scientific impact of the proposed chapter, the discussion of benchmarking battery models will continue focusing only on second-order models. However, in the literature, the main differences of the first- and second-order battery models were already detailed up to an extent. The added value of this chapter to the actual status of research is a different approach that focuses on the second-order battery model complexity and compromises when building the simulation programs.

It is known that nowadays, the simulators designed to emphasize phenomena especially in electrical engineering are more and more constructed using real-time platforms. By this, simulation sampling and accuracy of the results compared to those measured are mitigated seriously.

However, considering the large amount of data that needs to be recorded into matrixes for all the battery parameters becomes challenging when creating realtime simulation programs. Generally, such data becomes core in lookup tables (LUT). Loading LUT on a real-time processor becomes difficult as it requires space and decreases the sampling speed of the processor. For example, using fieldprogrammable gate arrays (FPGAs) becomes even more complicated in this approach, as those require add-on external flash memory, and by this the system becomes quite complicated as the interaction of the memory and the FPGA needs also to be additionally programmed.

Normally, a battery model architecture for the second-order model would require 6 LUTs as follows: one for the $\left(V_{o c}\right)$, one for the ohmic resistance $\left(R_{s}\right)$ and four others for $\left(R_{1}, R_{2}, C_{1}\right.$ and $\left.C_{2}\right)$.

Using an architecture as the one depicted in Figure 10 leads to high-accuracy, realistic results but also large memory necessity, long simulation time and nonrealistic sampling time. Hence, such design models are not at all justified to be implemented into real-time processors.

However, there is another approach that can be used in the case of real-time applications. Analyzing the parameter variations from Figure 11, although these are for the first-order circuit, the following statement is valid for the second-order circuit as well. It can be seen that around a SOC of $60 \%$, these parameters are quite constant. Sudden variations are recorded only around $10 \%$ and $100 \%$ of SOC. 

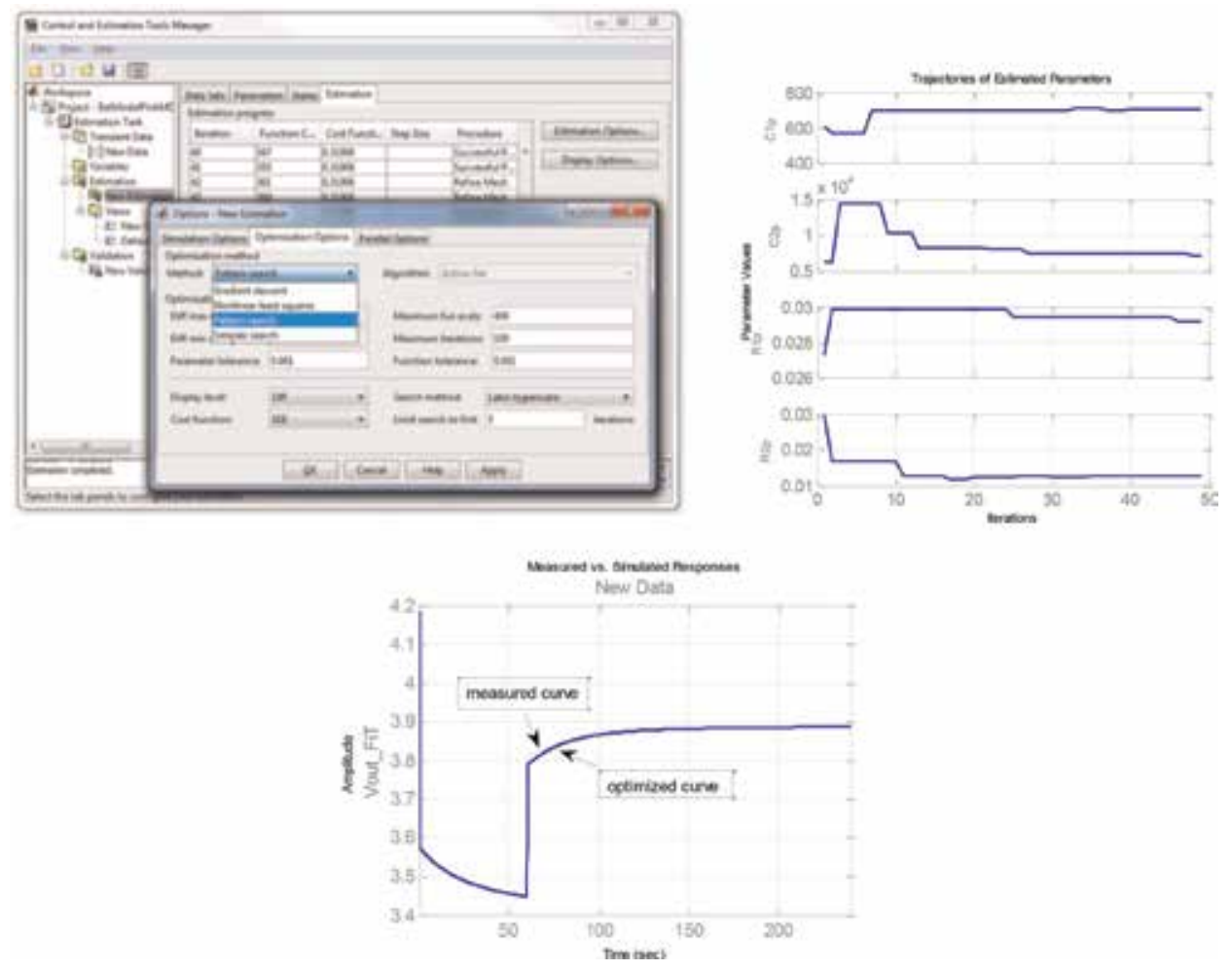

Figure 10.

The second-order battery model based on LUTs.

\section{Battery Data}

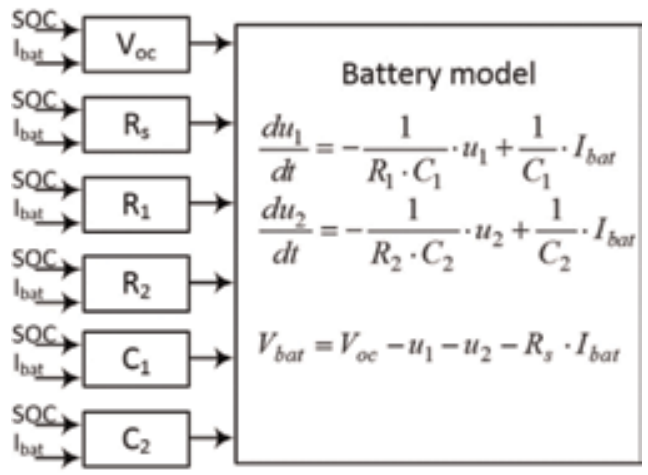

6xLUT

Figure 11.

The $R_{s}$, the $R_{p}$ and the $C_{p}$ functions of the SOC for the first-order model.

Hence, an approach that can simplify the modeling of a second-order circuit is to use instead of LUTs for each parameter constant values for the parameters recorded at a SOC of $60 \%$.

In Figure 12, the reduction of the model complexity of the second-order battery can be observed. Instead of 6 LUTs, only one remains, namely, the $\left(\mathrm{V}_{\mathrm{oc}}\right)$ function of the SOC. The rest of the parameters are constants, as mentioned before.

As it can be seen, the model is highly simple right now, and it contains only one LUT. The variation of the $\left(\mathrm{V}_{\mathrm{oc}}\right)$ depicted in Figure 6 that is identical for both the first- and the second-order models can be in fact described instead of a LUT with a 
polynomial function. With this the model, it becomes even more simple, without any LUT. In Figure 13, the results for the three different approaches, the fitted one, the optimized one and the one based on constants, all versus the measured one, are plotted. For all, the difference between the measured quantity and the one obtained from the three models is depicted in Figure 13 (bottom). Still, the largest error is returned by the fitted values, while the smallest error is given by using LUTs with optimized values. However, it is interesting to observe that when using constant values, fetched at a SOC of $60 \%$, the error is more than satisfactory. It has to be mentioned that the constant values were fetched out of the optimized data at the

\section{Battery Data}

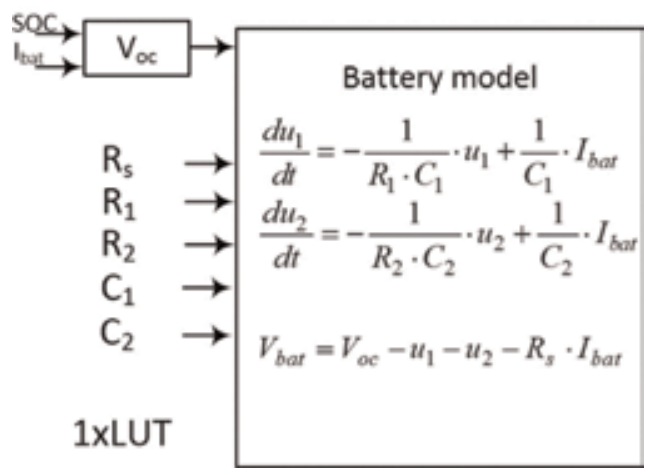

Figure 12.

The second-order battery model based on constants.
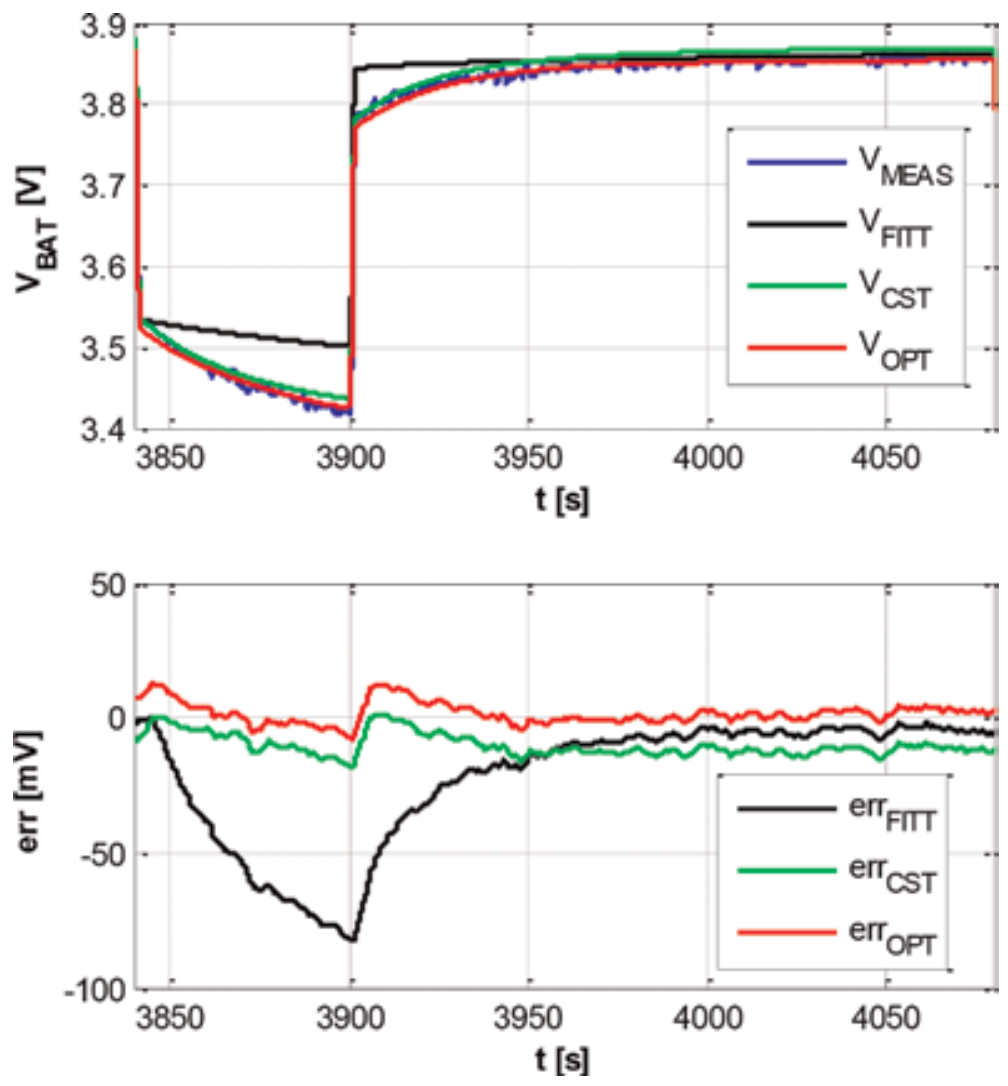

Figure 13.

The comparison of the three different modeling approaches for second-order ECM. 
Parameter Identification, Modeling and Testing of Li-Ion Batteries Used in Electric Vehicles DOI: http://dx.doi.org/10.5772/intechopen.89256

SOC of $60 \%$. The results detailed in Figure 13 and the explanations regarding this approach prove that one can simply build a second-order battery model that can perform simulations on a real-time processor, even a FPGA.

\subsection{Experimental validation of the proposed models}

As already stated, the focus of the chapter is around the second-order Li-ion battery modeling; hence, the proof of correct approach will be engaged still on second-order modeling compared to actual measurements.
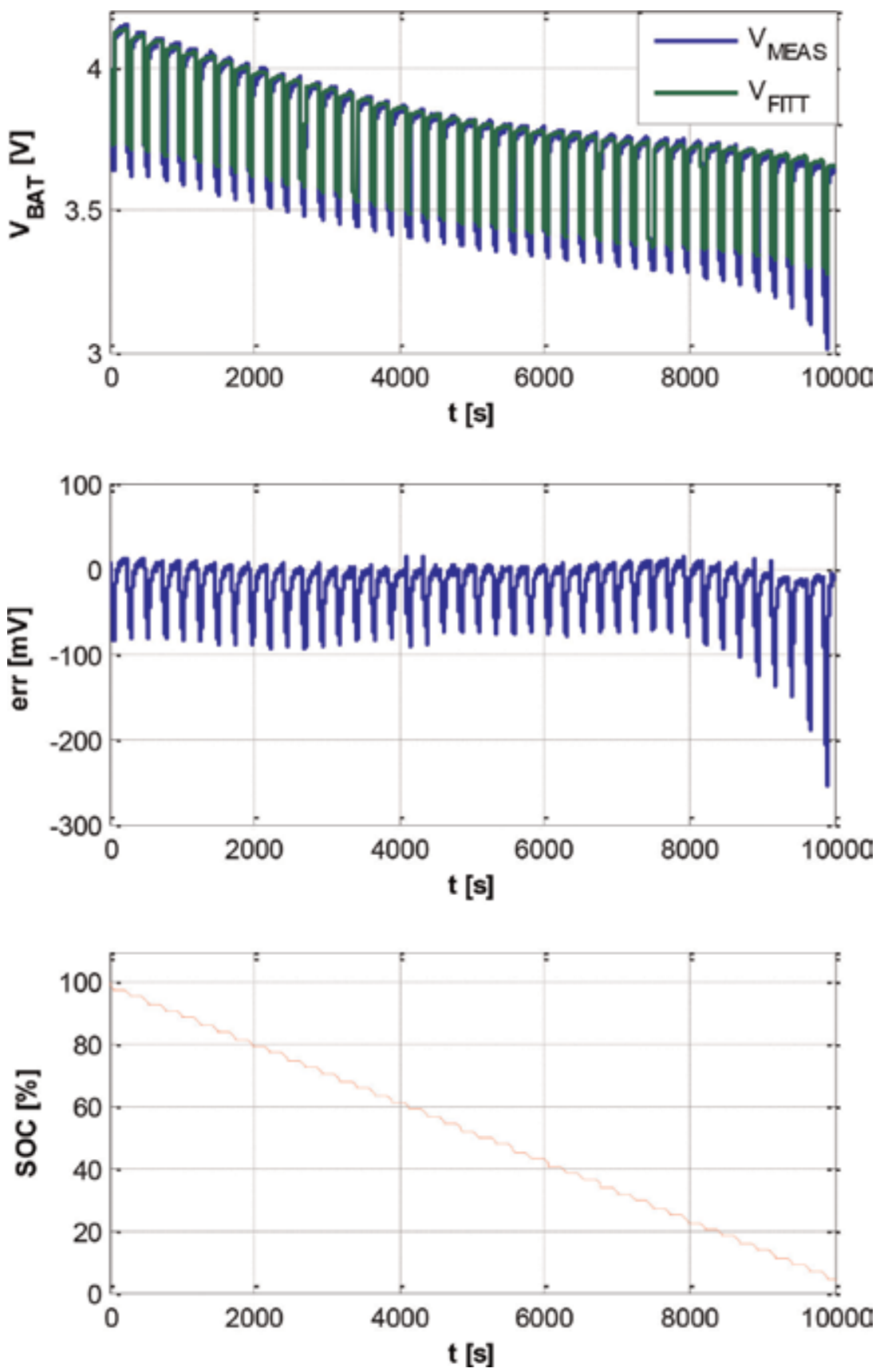

Figure 14 .

The measured vs. fitted parameters voltage (top), their difference (middle) and the SOC (bottom). 

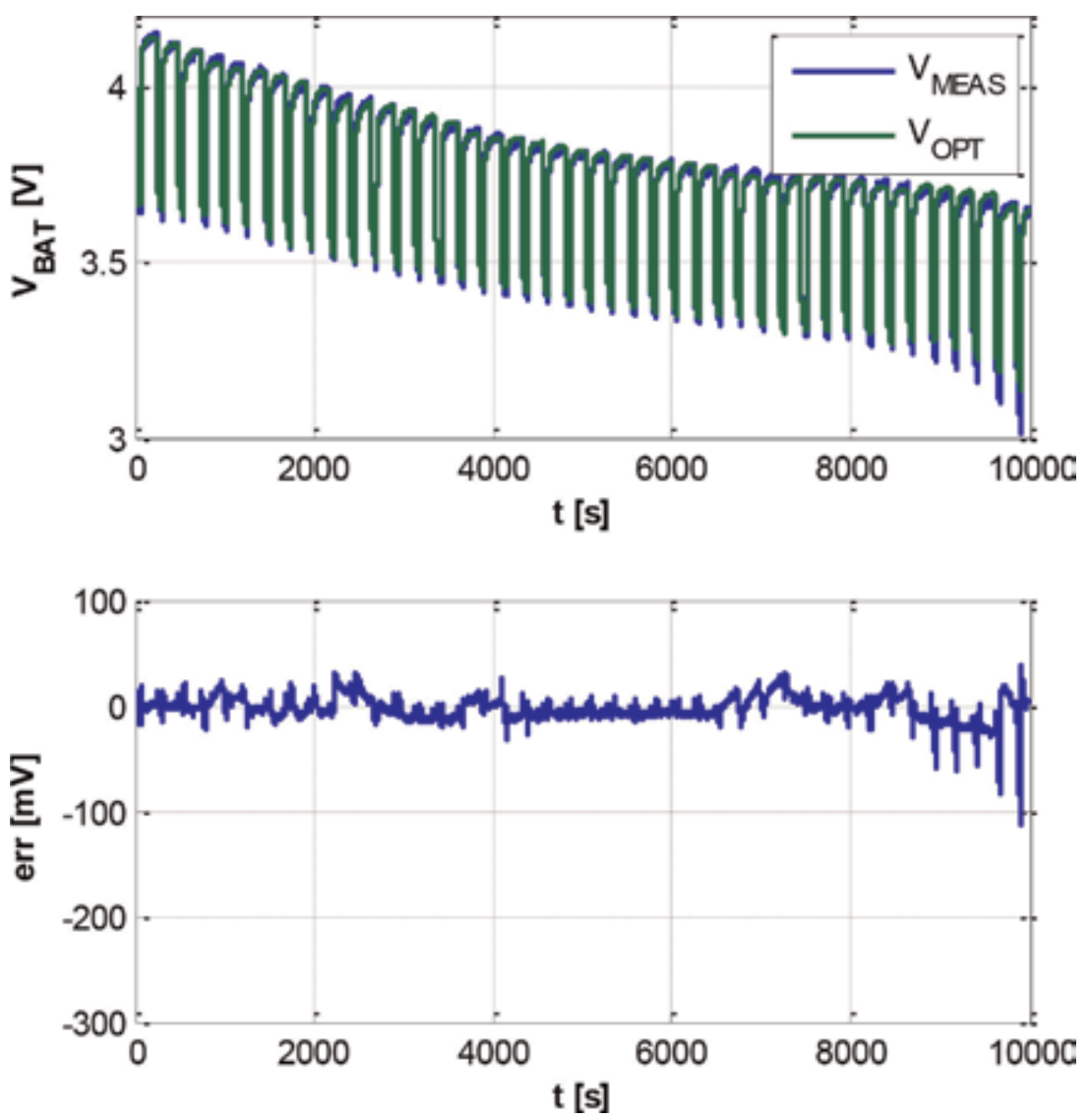

Figure 15.

The measured vs. optimized parameters voltage (top), their difference (bottom).

The simplest method of experimentally validating the analysis is to compare the measured discharge characteristic of the battery for the complete cycle with the results obtained from each modeling method.

In Figure 14, the validation of the model with fitted parameters is accomplished versus the actual measured battery voltage. To have a transparent comparison, the instantaneous error over the entire discharge range is computed. Generally the error is less than $100 \mathrm{mV}$ and increases to larger values especially when the SOC is less than 20\%. In Figure 15, the measured voltage is compared to the one obtained from simulations using the optimized parameters. Here, the error is consistently smaller than in the previous case, reaching $100 \mathrm{mV}$ only when the battery is completely discharged. The SOC depicted in Figure $\mathbf{1 4}$ is valid for Figures $\mathbf{1 5}$ and $\mathbf{1 6}$ as well. The last analyzed model is the one where the LUTs are replaced with constant values fetched at a SOC of 60\% (Figure 16). Analyzing the error one can state that this is larger than for the model with optimized values in LUTs but smaller than for the one with fitted values in LUTs.

The accuracy of the model based on constants is more than satisfactory and proves that designing such model can run both on computer and real-time platform simulators, reaching high accuracy and realistic behavior. 

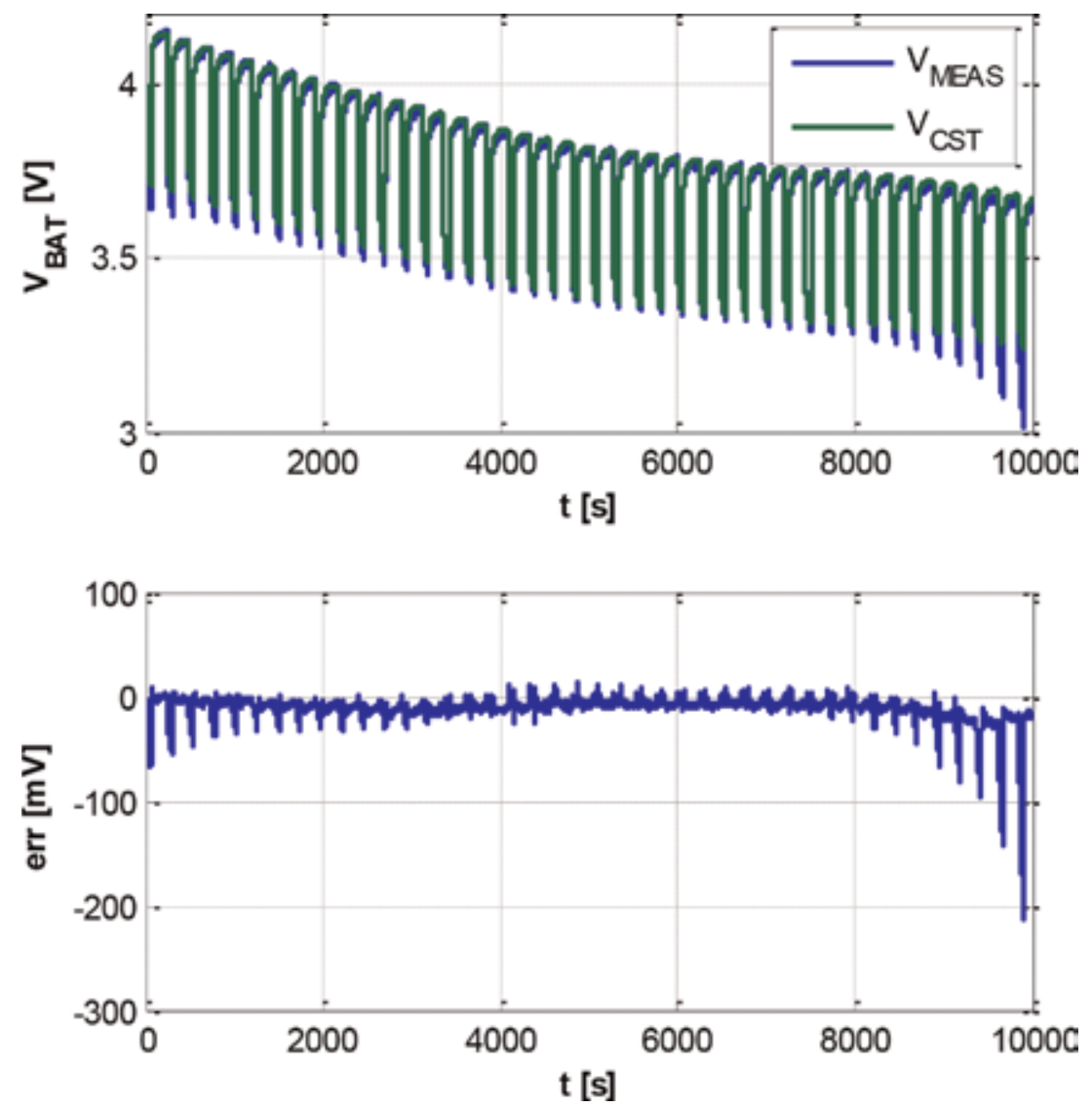

Figure 16.

The measured vs. constant parameters voltage (top), their difference (bottom).

\section{Conclusions}

The research conducted on battery modeling and simulation that is now a hot topic in many research facilities both in academia and industry sectors requires precision, accuracy and transparency but at the same time demands reaching all these with the assumption of simplicity. There are many publications that reach impressive accuracy when modeling battery cells but with the cost of high complexity and large data manipulation inside the model. When speaking about pure scientific impact, such models are more than beneficial to those involved in their development. However, when coping with industry applications, the precision is as important as is the simplicity. Hence, using the general architecture of high precision models and manipulating their parameters to avoid data overflow and keep the accuracy in satisfactory boundaries become a lucrative solution.

The authors proved in this chapter that one can reach with wise interpretation such solutions that are simple to use, are simple to design and offer the possibility to simulate quite close to the real behavior a Li-ion battery cell. 


\section{Author details}

Mircea Ruba*, Raul Nemeș, Sorina Ciornei and Claudia Marțiș Technical University of Cluj Napoca, Romania

*Address all correspondence to: mircea.ruba@emd.utcluj.ro

\section{IntechOpen}

(C) 2020 The Author(s). Licensee IntechOpen. This chapter is distributed under the terms of the Creative Commons Attribution License (http://creativecommons.org/licenses/ by/3.0), which permits unrestricted use, distribution, and reproduction in any medium, provided the original work is properly cited. (c) BY 


\section{References}

[1] DoyleThomas M, Fuller F, Newman J. Modeling of galvanostatic charge and discharge of the lithium/ polymer/insertion cell. Journal of the Electrochemical Society. 2018;140. DOI: 10.1149/2.1181811jes

[2] Wang QK, He y J, Shen JN, Ma ZF, Zhong GB. A unified modeling

framework for lithium-ion batteries: An artificial neural net-work based thermal coupled equivalent circuit model approach. Energy. 2017;138:118-132

[3] Chiang YH, Sean WY, Ke JC. Online estimation of internal resistance and open-circuit voltage of lithium-ion batteries in electric vehicles. Journal of Power Sources. 2011;196(8)

[4] Mathew M, Kong QH, McGrory J, Fowler M. Simulation of lithium ion battery replacement in a battery pack for application in electric vehicles. Journal of Power Sources. 2017;349:94-104

[5] Xia B, Sun Z, Zhang R, Lao Z. A cubature particle filter algorithm to estimate the state of the charge of lithium-ion batteries based on a second-order equivalent circuit model. Energies. 2017;10(4):457. DOI: 10.3390/ en10040457

[6] Zhang L, Peng H, Ning Z, Mu Z, Sun C. Comparative research on RC equivalent circuit models for lithiumion batteries of electric vehicles. Applied Sciences. 2017;7(10):1002. DOI: 10.3390/app7101002

[7] Klass V. Capturing lithium-ion battery dynamics with support vector machine-based battery model. Journal of Power Sources. 2015;298:92-101. DOI: 10.1016/j.jpowsour.2015.08.036

[8] Wang Q-K, He Y-J, Shen J-N, Ma Z-F, Zhong G-B. A unified modeling framework for lithium-ion batteries: An artificial neural network based thermal coupled equivalent circuit model approach. Energy. 2017;138: 118-132. DOI: $10.1016 /$ j. energy.2017.07.035

[9] Liu G, Lu L, Fu H, Hua J, Li J, Ouyang $\mathrm{M}$, et al. A comparative study of equivalent circuit models and enhanced equivalent circuit models of lithium-ion batteries with different model structures. In: 2014 IEEE Conference and Expo Transportation Electrification Asia-Pacific (ITEC Asia-Pacific), 31 Aug.-3 Sept. 2014

[10] Nemes R, Ciornei S, Ruba M, Hedesiu H, Martis C. Modeling and simulation of first-order Li-ion battery cell with experimental validation. In: The 8th International Conference on Modern Power Systems. 2019. in print

[11] Ruba M, Ciornei S, Nemeș R, Martis C. Detailed design of second order model of lithium-ion battery simulator based on experimental measurements. In: 2019 11th International Symposium on Advanced Topics in Electrical Engineering (ATEE), Bucharest, Romania. 2019. DOI: 10.1109/ATEE.2019.8724890

[12] Nemes RO, Ciornei SM, Ruba M, Martis C. Parameters identification using experimental measurements for equivalent circuit Lithium-Ion cell models. In: 2019 11th International Symposium on Advanced Topics in Electrical Engineering (ATEE), Bucharest, Romania. 2019. DOI: 10.1109/ATEE.2019.8724878 



\title{
Permanent-Magnet Synchronous Machine Drives
}

\author{
Adhavan Balashanmugham and Mockaisamy Maheswaran
}

\begin{abstract}
The permanent-magnet synchronous machine (PMSM) drive is one of best choices for a full range of motion control applications. For example, the PMSM is widely used in robotics, machine tools, actuators, and it is being considered in highpower applications such as industrial drives and vehicular propulsion. It is also used for residential/commercial applications. The PMSM is known for having low torque ripple, superior dynamic performance, high efficiency and high power density. Section 1 deals with the introduction of PMSM and how it is evolved from synchronous motors. Section 2 briefly discusses about the types of PMSM. Section 3 tells about the assumptions in PMSM for modeling of PMSM and it derives the equivalent circuit of PMSM. In Section 4, permanent magnet synchronous motor drive system is briefly discussed with explanation of each blocks in the systems. Section 5 reveals about the control techniques of PMSM like scalar control, vector control and simulation of PMSM driven by field-oriented control using fuzzy logic control with space vector modulation for minimizing torque ripples. PMSM control with and without rotor position sensors along with different control techniques for controlling various parameters of PMSM for different applications is presented in Section 6.
\end{abstract}

Keywords: types of PMSM, modelling of PMSM, construction of PMSM drive systems, control techniques of PMSM, advanced topics in PMSM drives sensored control and sensorless control

\section{Introduction}

The electric motors are electromechanical machines, which are used for the conversion of electrical energy into mechanical energy. The foremost categories of AC motors are asynchronous and synchronous motors. The asynchronous motors are called singly excited machines, that is, the stator windings are connected to AC supply whereas the rotor has no connection from the stator or to any other source of supply. The power is transferred from the stator to the rotor only by mutual induction, owing to which the asynchronous motors are called as induction machines.

The synchronous motors require AC supply for the stator windings and DC supply for the rotor windings. The motor speed is determined by the AC supply frequency and the number of poles of the synchronous motor, the rotor rotates at the speed of the stator revolving field at synchronous speed, which is constant. The variations in mechanical load within the machine's rating will not affect the motor's synchronous speed [1]. 
One of the types of synchronous motor is the PMSM. The PMSM consists of conventional three phase windings in the stator and permanent magnets in the rotor. The purpose of the field windings in the conventional synchronous machine is done by permanent magnets in PMSM. The conventional synchronous machine requires AC and DC supply, whereas the PMSM requires only AC supply for its operation. One of the greatest advantages of PMSM over its counterpart is the removal of dc supply for field excitation as discussed in [2].

The development of PMSM has happened due to the invention of novel magnetic materials and rare earth materials. PMSM give numerous advantages in scheming recent motion management systems. Energy efficient PMSM are designed due to the availability of permanent magnet materials of high magnetic flux density.

In synchronous motors the rotor rotates at the speed of stator revolving field. The speed of the revolving stator field is called as synchronous speed. The synchronous speed $\left(\omega_{s}\right)$ can be found by the frequency of the stator input supply $\left(f_{s}\right)$, and the number of stator pole pairs $(p)$. The stator of a three phase synchronous motor consists of distributed sine three phase winding, whereas the rotor consists of the same number of $p$-pole pairs as stator, excited by permanent magnets or a separate DC supply source as given in [3].

When the synchronous machine is excited with a three phase AC supply, a magnetic field rotates at synchronous speed develops in the stator. The synchronous speed of this rotating magnetic field is shown by the Eq. (1).

$$
\mathrm{N}=\left(120 f_{s}\right) / \mathrm{P} \mathrm{rpm}
$$

where $\mathrm{N}$, synchronous speed, $f_{s}$, frequency of AC supply in $\mathrm{Hz} ; P$, number of poles; $p$, pole pairs and it is given by $(P / 2)$.

\section{Types of PMSM}

The PMSM are classified based on the direction of field flux are as follows,

\section{Radial field}

\section{Axial field}

In radial field, the flux direction is along the radius of the machine. The radial field permanent magnet motors are the most commonly used. In axial field, the flux direction is parallel to the rotor shaft. The axial field permanent magnet motors are presently used in a variety of numerous applications because of their higher power density and quick acceleration.

The permanent magnets can be placed in many different ways on the rotor of PMSM as discussed in [3, 4]. Figures 1 and 2 show the permanent magnets mounted on the surface of the outer periphery of rotor laminations. This type of arrangement provides the highest air gap flux density, but it has the drawback of lower structural integrity and mechanical robustness. Machines with this arrangement of magnets are known as Surface mount PMSMs.

One other types of placing the permanent magnets in the rotor, is embedding the permanent magnets inside the rotor laminations. This type of machine construction is generally referred to as Interior PMSM and it is shown in Figures $\mathbf{3}$ and $\mathbf{4}$.

The development of this arrangement is more difficult than the surface mount or inset magnet permanent magnet rotors. The inset permanent magnet rotor construction has the advantages of both the surface and interior permanent magnet 


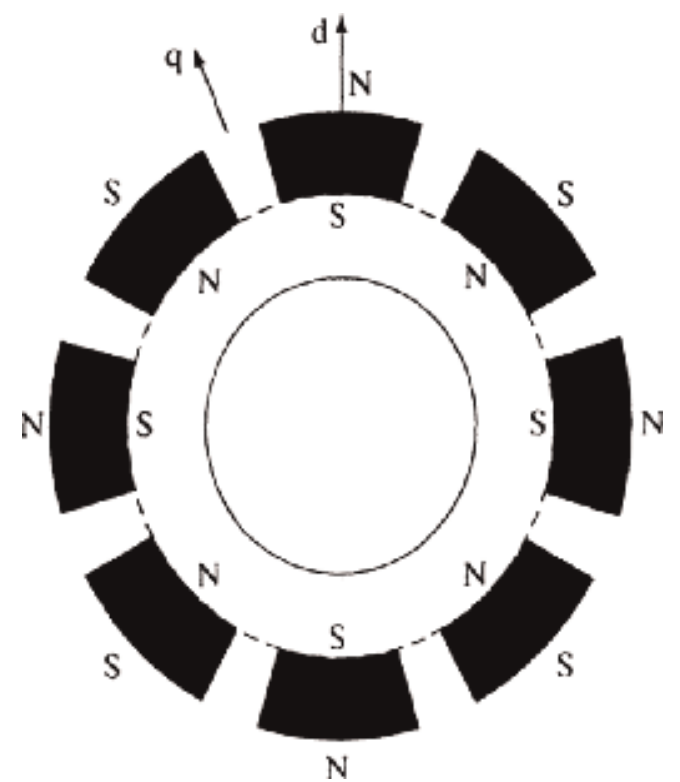

Figure 1.

Surface permanent magnet.

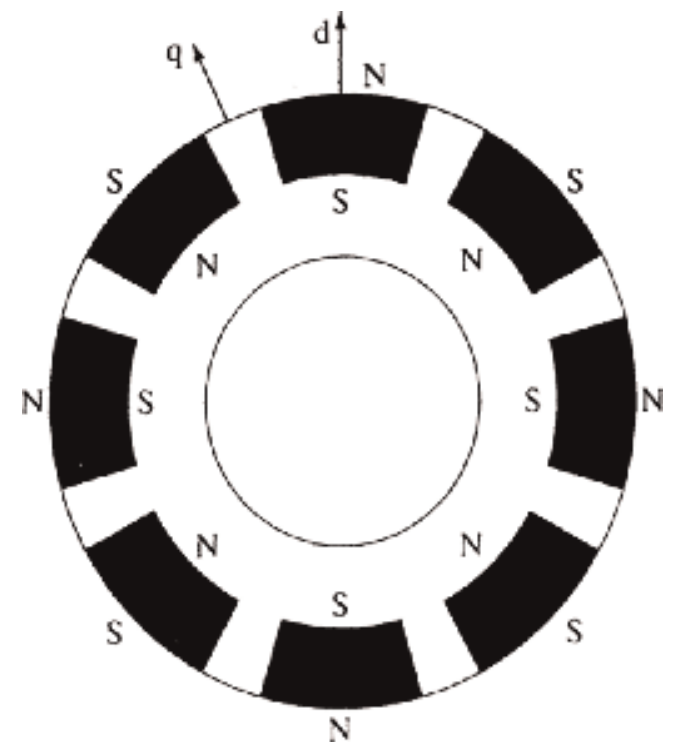

Figure 2.

Surface inset permanent magnet.

rotor arrangements by easier construction and mechanical robustness, with a high ratio between the quadrature and direct-axis inductances, respectively.

The surface PMSM with radial flux are generally applied for applications which require low speed operations. These machines have the advantage of high power density than the other types of PMSM. The interior PMSM are used for applications which require high speed.

The principle of operation is identical for all the types of PMSM, in spite of the types of mounting the permanent magnets in the rotor. 


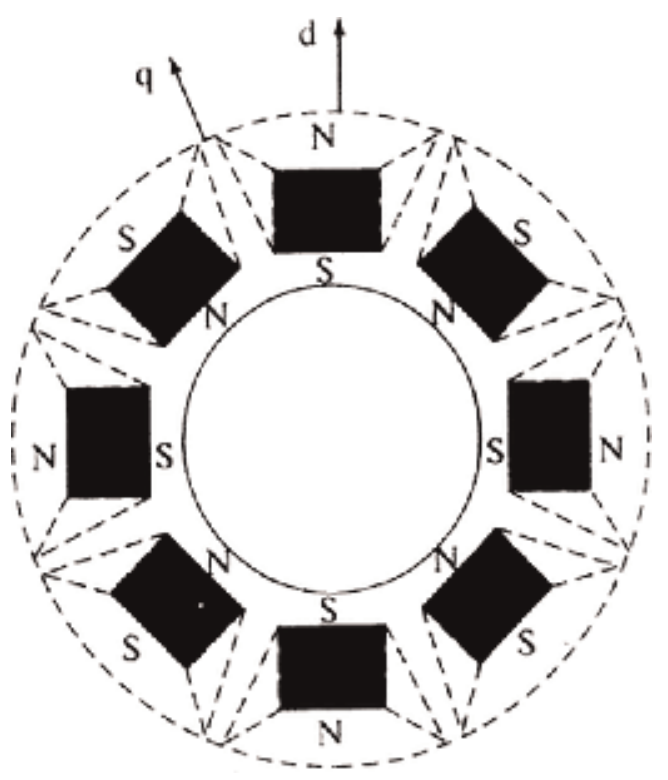

Figure 3.

Interior permanent magnet.

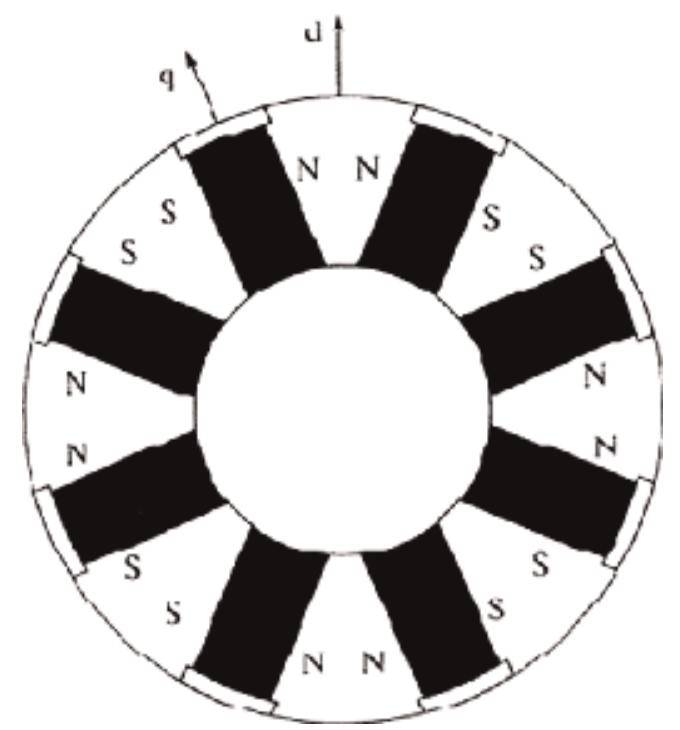

Figure 4.

Interior permanent magnet with circumferential orientation.

The important significance of the type of mounting the permanent magnets on the rotor is the variation in direct axes and quadrature axes inductance values, which is explained below. The primary path of the flux through the permanent magnets rotor is the direct axis. The stator inductance when measured in the position of permanent magnets aligned with stator winding is called as direct axis inductance. The quadrature axis inductance is measured by rotating the magnets from the already aligned position (direct axis) by $90^{\circ}$, in this position the iron (inter polar area of the rotor) sees the stator flux. The flux density of the permanent magnet materials is presently high and its permeability is almost equal to that of the 
air, such that the air gap between the rotor and stator of PMSM can be treated as an extension of permanent magnet thickness. The reluctance of direct axis is always greater than the quadrature axis reluctance, since the effectual air gap of the direct axis is several times that of the real air gap looked by the quadrature axis.

The significance of such an uneven reluctance is that the direct axis inductance is greater than the quadrature axis inductance and it is shown in Eq. (2).

$$
\mathrm{L}_{\mathrm{d}}>\mathrm{L}_{\mathrm{q}}
$$

where $L_{d}$ is the inductance along the direct to the magnet axis and $L_{q}$ is the inductance along the axis in quadrature to the magnet axis.

\section{Modeling of PMSM}

For proper simulation and analysis of the system, a complete modelling of the drive model is essential. The motor axis has been developed using d-q rotor reference frame theory as shown in Figure 5, as given [5]. At any particular time t, the rotor reference axis makes an angle $\theta_{\mathrm{r}}$ with the fixed stator axis and the rotating stator $\mathrm{mmf}$ creates an angle $\alpha$ with the rotor $\mathrm{d}$ axis. It is viewed that at any time t, the stator mmf rotates at the same speed as that of the rotor axis.

The required assumptions are obtained for the modelling of the PMSM without damper windings.

1. Saturation is neglected.

2. Induced EMF is sinusoidal in nature.

3. Hysteresis losses and Eddy current losses are negligible.

4. No field current dynamics.

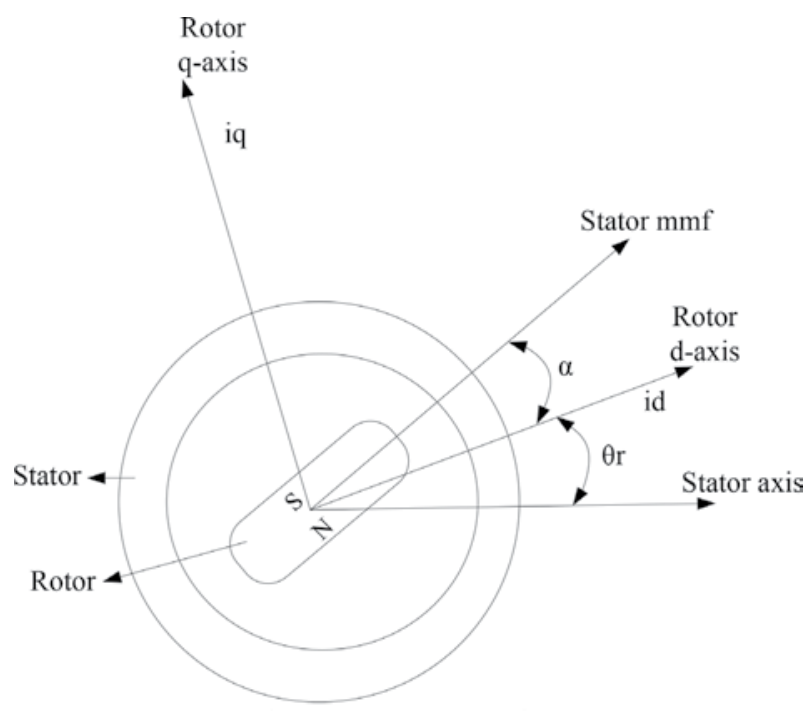

Figure 5 .

Motor axis. 
Voltage equations from the model are given by,

$$
\begin{aligned}
& \mathrm{V}_{\mathrm{q}}=\mathrm{R}_{\mathrm{s}} \mathrm{i}_{\mathrm{q}}+\omega_{\mathrm{r}} \lambda_{\mathrm{d}}+\rho \lambda_{\mathrm{q}} \\
& \mathrm{V}_{\mathrm{d}}=\mathrm{R}_{\mathrm{s}} \mathrm{i}_{\mathrm{d}}-\omega_{\mathrm{r}} \lambda_{\mathrm{q}}+\rho \lambda_{\mathrm{d}}
\end{aligned}
$$

Flux linkages are given by,

$$
\begin{gathered}
\lambda_{\mathrm{q}}=\mathrm{L}_{\mathrm{q}} \mathrm{i}_{\mathrm{q}} \\
\lambda_{\mathrm{q}}=\mathrm{L}_{\mathrm{q}} \mathrm{i}_{\mathrm{q}}+\lambda_{\mathrm{f}}
\end{gathered}
$$

Substituting Eq. (5) and Eq. (6) into Eq. (3) and Eq. (4)

$$
\begin{aligned}
& \mathrm{V}_{\mathrm{q}}=\mathrm{R}_{\mathrm{s}} \mathrm{i}_{\mathrm{q}}+\omega_{\mathrm{r}}\left(\mathrm{L}_{\mathrm{d}} \mathrm{i}_{\mathrm{d}}+\lambda_{\mathrm{f}}\right)+\rho \mathrm{L}_{\mathrm{d}} \mathrm{i}_{\mathrm{d}} \\
& \mathrm{V}_{\mathrm{d}}=\mathrm{R}_{\mathrm{s}} \mathrm{i}_{\mathrm{d}}-\omega_{\mathrm{r}} \mathrm{L}_{\mathrm{q}} \mathrm{i}_{\mathrm{q}}+\rho\left(\mathrm{L}_{\mathrm{d}} \mathrm{i}_{\mathrm{d}}+\lambda_{\mathrm{f}}\right)
\end{aligned}
$$

Arranging Eq. (7) and Eq. (8) in matrix form,

$$
\left(\begin{array}{c}
\mathrm{V}_{\mathrm{q}} \\
\mathrm{V}_{\mathrm{d}}
\end{array}\right)=\left(\begin{array}{cc}
\mathrm{R}_{\mathrm{s}}+\rho \mathrm{L}_{\mathrm{q}} & \omega_{\mathrm{r}} \mathrm{L}_{\mathrm{d}} \\
-\omega_{\mathrm{r}} \mathrm{L}_{\mathrm{q}} & \mathrm{R}_{\mathrm{s}}+\rho \mathrm{L}_{\mathrm{d}}
\end{array}\right)\left(\begin{array}{c}
\mathrm{i}_{\mathrm{q}} \\
\mathrm{i}_{\mathrm{d}}
\end{array}\right)+\left(\begin{array}{c}
\omega_{\mathrm{r}} \lambda_{\mathrm{f}} \\
\rho \lambda_{\mathrm{f}}
\end{array}\right)
$$

The developed torque motor is being given by,

$$
\mathrm{T}_{\mathrm{e}}=\frac{3}{2}\left(\frac{\mathrm{P}}{2}\right)\left(\lambda_{\mathrm{d}} \mathrm{i}_{\mathrm{q}}-\lambda_{\mathrm{q}} \mathrm{i}_{\mathrm{d}}\right)
$$

The mechanical torque equation is,

$$
T_{e}=T_{L}+B \omega_{m}+J \frac{d \omega_{m}}{d t}
$$

Solving for the rotor mechanical speed form Eq. (11)

$$
\omega_{\mathrm{m}}=\int\left(\frac{\mathrm{T}_{\mathrm{e}}-\mathrm{T}_{\mathrm{L}}-\mathrm{B} \omega_{\mathrm{m}}}{\mathrm{J}}\right) \mathrm{dt}
$$

and

$$
\omega_{\mathrm{m}}=\omega_{\mathrm{r}}\left(\frac{2}{\mathrm{P}}\right)
$$

In the above equations $\omega_{\mathrm{r}}$ is the rotor electrical speed, $\omega_{\mathrm{m}}$ is the rotor mechanical speed.

\subsection{Parks transformation and dynamic d-q modeling}

The dynamic d-q modelling of the system is used for the study of motor during transient state and as well as in the steady state conditions. It is achieved by converting the three phase voltages and currents to dqo axis variables by using the Parks transformation [4].

Converting the phase voltages variables $\mathrm{V}_{\mathrm{abc}}$ to $\mathrm{V}_{\mathrm{dqo}}$ variables in rotor reference frame axis are illustrated in the equations, 


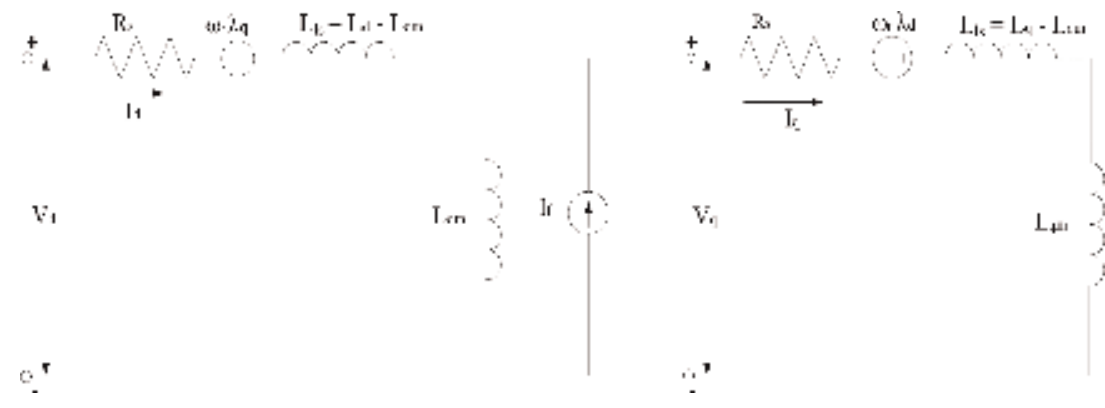

Figure 6.

Equivalent circuit of PMSM without damper windings.

$$
\left[\begin{array}{c}
\mathrm{V}_{\mathrm{q}} \\
\mathrm{V}_{\mathrm{d}} \\
\mathrm{V}_{\mathrm{o}}
\end{array}\right]=\frac{2}{3}\left[\begin{array}{ccc}
\cos \theta_{\mathrm{r}} & \cos \left(\theta_{\mathrm{r}}-120\right) & \cos \left(\theta_{\mathrm{r}}+120\right) \\
\sin \theta_{\mathrm{r}} & \sin \left(\theta_{\mathrm{r}}-120\right) & \sin \left(\theta_{\mathrm{r}}+120\right) \\
1 / 2 & 1 / 2 & 1 / 2
\end{array}\right]\left[\begin{array}{c}
\mathrm{V}_{\mathrm{a}} \\
\mathrm{V}_{\mathrm{b}} \\
\mathrm{V}_{\mathrm{c}}
\end{array}\right]
$$

Convert $\mathrm{V}_{\mathrm{dqo}}$ to $\mathrm{V}_{\mathrm{abc}}$

$$
\left[\begin{array}{c}
\mathrm{V}_{\mathrm{a}} \\
\mathrm{V}_{\mathrm{b}} \\
\mathrm{V}_{\mathrm{c}}
\end{array}\right]=\frac{2}{3}\left[\begin{array}{ccc}
\cos \theta_{\mathrm{r}} & \sin \theta_{\mathrm{r}} & 1 \\
\cos \left(\theta_{\mathrm{r}}-120\right) & \sin \left(\theta_{\mathrm{r}}-120\right) & 1 \\
\cos \left(\theta_{\mathrm{r}}+120\right) & \sin \left(\theta_{\mathrm{r}}+120\right) & 1
\end{array}\right]\left[\begin{array}{c}
\mathrm{V}_{\mathrm{q}} \\
\mathrm{V}_{\mathrm{d}} \\
\mathrm{V}_{\mathrm{o}}
\end{array}\right]
$$

\subsection{Equivalent circuit of PMSM}

Equivalent circuit is essential for the proper simulation and designing of the motor. It is achieved and derived from the d-q modelling of the motor using the voltage equations of the stator. From the assumption, rotor $\mathrm{d}$ axis flux is represented by a constant current source which is described through the following equation,

$$
\lambda_{\mathrm{f}}=\mathrm{L}_{\mathrm{dm}} \mathrm{i}_{\mathrm{f}}
$$

where $\lambda_{\mathrm{f}}$, field flux linkage; $\mathrm{L}_{\mathrm{dm}}$, $\mathrm{d}$-axis magnetizing inductance; $\mathrm{i}_{\mathrm{f}}$, equivalent permanent magnet field current.

Figure 6 shows the equivalent circuit of PMSM without damper windings.

\section{Permanent magnet synchronous motor drive system}

The motor drive essentially consists of four main components such as the PMSM, the inverter, the main control unit and the position sensor. Interconnections of the components are shown in Figure 7.

\subsection{Inverter}

For variable frequency and magnitude, voltage source inverters are devices which convert the constant $\mathrm{DC}$ voltage level to variable $\mathrm{AC}$ voltage. As specified in the function, these inverters are commonly used in adjustable speed drives. 


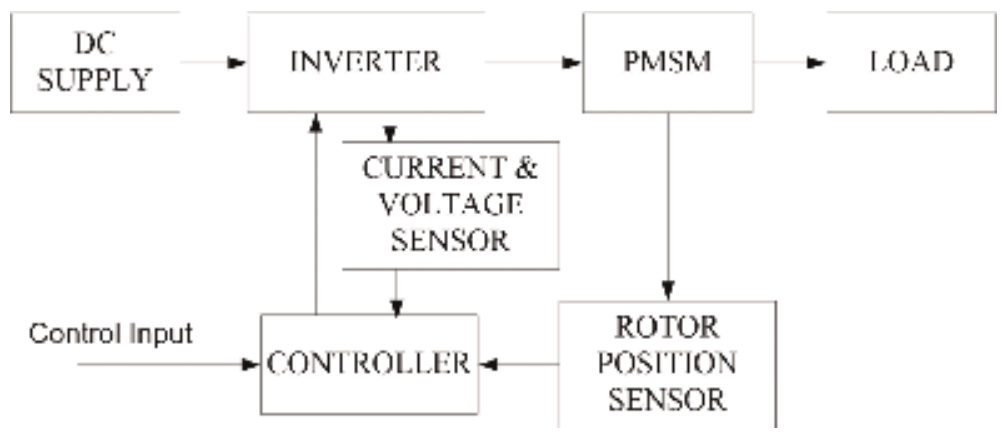

Figure 7.

Components permanent magnet synchronous motor drive.

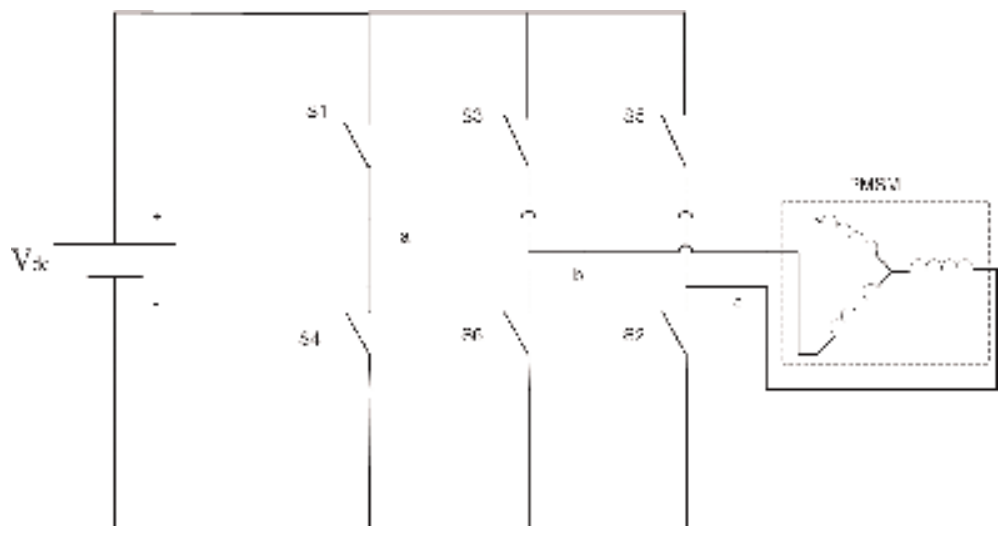

Figure 8.

Voltage source inverter with DC supply and load (PMSM).

Figure 8 shows a voltage source inverter with a supply voltage $V_{d c}$ and with six switches. The frequency of the AC voltage can be variable or constant based on the applications $[2,6]$.

Three phase inverters consist of a DC voltage source and six power ON/OFF switches connected to the PMSM as shown in Figure 8. Selection of the inverter switches must be carefully done based on the necessities of operation, ratings and the application. There are several devices available in the market and these are thyristors, bipolar junction transistors (BJTs), MOS field effect transistors (MOSFETs), insulated gate bipolar transistors (IGBTs) and gate turn off thyristors (GTOs). It has been inferred that MOSFETs and IGBTs are preferred in the industry because of its advantages that the MOS gating permits high power gain and control advantages. MOSFET is considered to be universal power ON/OFF device for low power and low voltage applications, whereas IGBT has wide acceptance in the motor drive applications and other application in the low and medium power range. The power devices when used in motor drives applications require an inductive motor current path provided by antiparallel diodes when the switch is turned off.

\section{Control techniques of PMSM}

Many techniques based on both motor designs and control techniques that have been proposed in literature to diminish the torque ripples in the PMSM (Figure 9). 


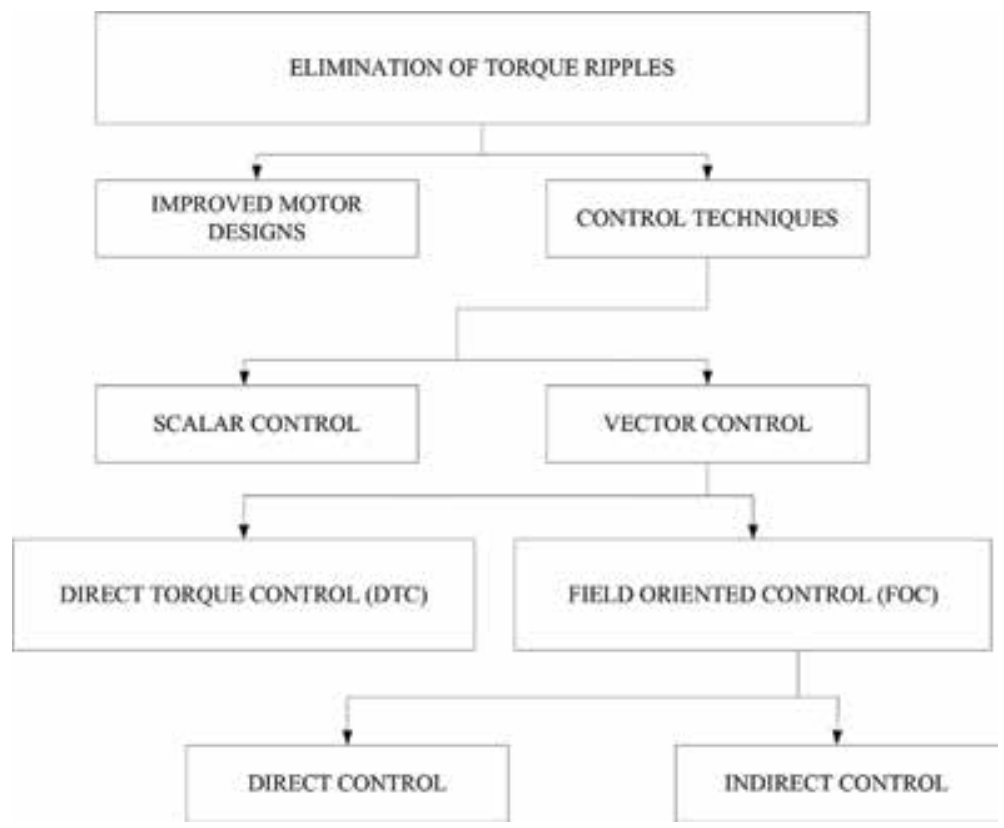

Figure 9.

Classification of the various control techniques.

\subsection{Scalar control}

One way of controlling AC motors for variable speed applications is through the open loop scalar control, which represents the most popular control strategy of squirrel cage AC motors. It is presently used in applications where information about the angular speed need not be known. It is suitable for a wide range of drives as it ensures robustness at the cost of reduced dynamic performance. Typical applications are pump and fan drives and low-cost drives. The main idea of this method is the variation of the supply voltage frequency inattentively from the shaft response (position, angular speed). The magnitude of the supply voltage is changed according to the frequency in a constant ratio. The motor is then in the condition where the magnetic flux represents the nominal value and the motor is neither over excited nor under excited. The major advantage of this simple method is running in a sensorless mode because the control algorithm does not need information about the angular speed or actual rotor position. On the contrary, the significant disadvantages are the speed dependence on the external load torque, mainly for PMSM, and the reduced dynamic performances.

\subsection{Vector control}

The vector control of PMSM allows separate closed loop control of both the flux and torque, thereby achieving a similar control structure to that of a separately excited DC machine, as discussed in [7].

\subsubsection{Direct torque control (DTC)}

The DTC is one of the high performance control strategies for the control of AC machine. In a DTC drive applications, flux linkage and electromagnetic torque are controlled directly and independently by the selection of optimum inverter switching modes of operation. To acquire a faster torque output, low inverter switching frequency and low harmonic losses in the model, the selection is made to restrict the flux 
linkages and electromagnetic torque errors within the respective flux and torque hysteresis bands. The required optimal switching vectors can be selected by using the optimum switching voltage vector look-up table. This can be obtained by simple physical considerations involving the position of the stator-flux linkage space vector, the available switching vectors, and the required torque flux linkage.

\subsubsection{Field oriented control (FOC) of PMSM}

For the control of PM motors, FOC technique is used for synchronous motor to evaluate as a DC motor. The stator windings of the motor are fed by an inverter that generates a variable frequency variable voltage scheme. Instead of controlling the inverter frequency independently, the frequency and phase of the output wave are controlled using a position sensor.

FOC was invented in the beginning of 1970 s and it demonstrates that an induction motor or synchronous motor could be controlled like a separately excited DC motor by the orientation of the stator mmf or current vector in relation to the rotor flux to achieve a desired objective. For the motor to behave like a DC motor, the control needs knowledge of the position of the instantaneous rotor flux or rotor position of permanent magnet motor. This needs a resolver or an absolute optical encoder. Knowing the position, the three phase currents can be calculated. Its calculation using the current matrix depends on the control desired. Some control options are constant torque and flux weakening. These options are based in the physical limitation of the motor and the inverter. The limit is established by the rated speed of the motor, at which speed the constant torque operation finishes and the flux weakening starts as shown in Figure 10 as shown in [7]. From the literature it has been found that the best control for PMSM to make it to behave like a DC motor using decoupling control is known as vector control or field oriented control. The torque components of flux and currents in the motor are separated by the vector control through its stator excitation.

From the dynamic model of the PMSM, the vector control is derived.

Assuming the line currents as input signals,

$$
\begin{gathered}
\mathrm{i}_{\mathrm{a}}=\mathrm{I}_{\mathrm{m}} \sin \left(\omega_{\mathrm{r}} \mathrm{t}+\alpha\right) \\
\mathrm{i}_{\mathrm{b}}=\mathrm{I}_{\mathrm{m}} \sin \left(\omega_{\mathrm{r}} \mathrm{t}+\alpha-\frac{2 \pi}{3}\right) \\
\mathrm{i}_{\mathrm{c}}=\mathrm{I}_{\mathrm{m}} \sin \left(\omega_{\mathrm{r}} \mathrm{t}+\alpha+\frac{2 \pi}{3}\right)
\end{gathered}
$$

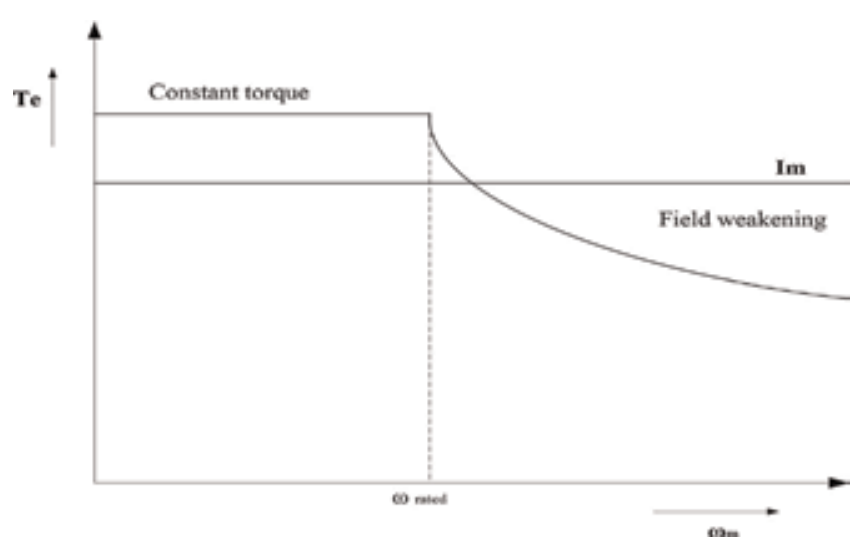

Figure 10.

Steady state torque versus speed. 
Writing the above Eq. (17) to Eq. (19) in the matrix form,

$$
\left(\begin{array}{c}
i_{a} \\
i_{b} \\
i_{c}
\end{array}\right)=\left(\begin{array}{c}
\cos \left(\omega_{r} t+\alpha\right) \\
\cos \left(\omega_{r} t+\alpha-\frac{2 \pi}{3}\right) \\
\cos \left(\omega_{r} t+\alpha+\frac{2 \pi}{3}\right)
\end{array}\right)(I m)
$$

where $\alpha$ is the angle between the rotor field and stator current phasor, $\omega_{r}$ is the electrical rotor speed.

Using the Park's transformation, the currents obtained in the previous cycle are transformed to the rotor reference frame axis with the rotor speed $\omega_{r}$. Since $\alpha$ is fixed for a given load torque, the $\mathrm{q}$ and $\mathrm{d}$ axis currents are fixed in the rotor reference frames. These constant values are made similar to the armature and field currents in the separately excited DC machine. The q axis current is distinctly equivalent to the armature current of the DC machine. The $\mathrm{d}$ axis current is field current, but not in its entirety. It is only a partial field current; the other part is contributed by the equivalent current source representing the permanent magnet field. Thus, the $q$ axis current is known as the torque producing component and the $\mathrm{d}$ axis current is called the flux producing component of the stator currents.

Substituting Eq. (20) in Eq. (14) and obtaining $i_{d}$ and $i_{q}$ in terms of $I_{m}$ as follows,

$$
\left(\begin{array}{c}
i_{\mathrm{q}} \\
i_{d}
\end{array}\right)=I_{m}\left(\begin{array}{c}
\sin \alpha \\
\cos \alpha
\end{array}\right)
$$

Using Eq. (3), Eq. (4), Eq. (10) and Eq. (21) the electromagnetic torque equation is obtained as given below,

$$
\mathrm{T}_{\mathrm{e}}=\frac{3}{2} \cdot \frac{\mathrm{P}}{2}\left[\frac{1}{2}\left(\mathrm{~L}_{\mathrm{d}}-\mathrm{L}_{\mathrm{q}}\right) \mathrm{I}_{\mathrm{m}}^{2} \sin 2 \alpha+\lambda_{\mathrm{f}} \mathrm{I}_{\mathrm{m}} \sin \alpha\right]
$$

where $L_{d}$ and $L_{q}$ are the $d$ and $q$ axis synchronous inductances. Each of the two terms in the equation has a useful physical interpretation. The first "magnet" torque term is independent of $i_{d}$ but is directly proportional to the stator current component $\mathrm{i}_{\mathrm{q}}$. In contrast, the second reluctance torque term is proportional to the $\mathrm{i}_{\mathrm{d}}$ and $\mathrm{i}_{\mathrm{q}}$ current component product and to the difference of the inductance values.

As Eq. (22) shows that the torque depends on the rotor type and its inductances $\mathrm{L}_{\mathrm{d}}, \mathrm{L}_{\mathrm{q}}$ and on permanent magnets mounted on the rotor. The non-salient PMSM have surface mounted magnets on the rotor and the reluctance term disappears since $\mathrm{L}_{\mathrm{q}}$ equals $\mathrm{L}_{\mathrm{d}}$. On the contrary, the electromagnetic torque is more dominated by the reluctance component when permanent magnets are interior mounted and the rotor's saliency causes a difference in $\mathrm{L}_{\mathrm{q}}$ and $\mathrm{L}_{\mathrm{d}}$.

\subsubsection{Simulation of permanent magnet synchronous motor driven by field oriented control using fuzzy logic control with space vector modulation for minimizing torque ripples}

One of the major disadvantages of the PMSM drive is the torque ripple produced which can be attributed to the following sources:

1. mutual torque, due to the interaction of the rotor field and stator currents;

2. reluctance torque, due to rotor saliency;

3. cogging torque, due to the existence of stator slots. 
In this section presents an application of fuzzy logic control to denigrate the torque ripple associated with the field oriented control when used in control of a PMSM. a SVPWM based FOC with fuzzy logic control is proposed to produce an effective selection of the stator voltage vector to obtain smooth torque performance. The significant advantages of space vector modulation are the ease of microprocessor implementation. Also, one advantage of FOC is that it increases efficiency, letting smaller motors replace larger ones without sacrificing torque and speed. Another advantage is that it offers higher, more dynamic performance in the case of speed and torque controlled ac drives.

The block diagram of proposed FOC with the fuzzy logic based controller for the PMSM drive is shown in Figure 11.

The fuzzy logic controller (FLC) executes the rule based taking the inputs and gives the output by defuzzification. The inputs are torque error (e) and change in torque error (ce) and the output is torque limit $\left(\mathrm{T}^{*}\right)$ which is equivalent to $i_{\text {sqref. }}$. The dq projections of the stator currents are then compared to their reference values $i_{\text {sqref }}$ and $i_{\text {sdref }}=0$ (to get maximum torque, set to zero if there is no field weakening) and corrected by means of PI current controllers. The outputs of the current controller are passed through the inverse Park transform and a new stator voltage vector is impressed to the motor using the SVPWM technique.

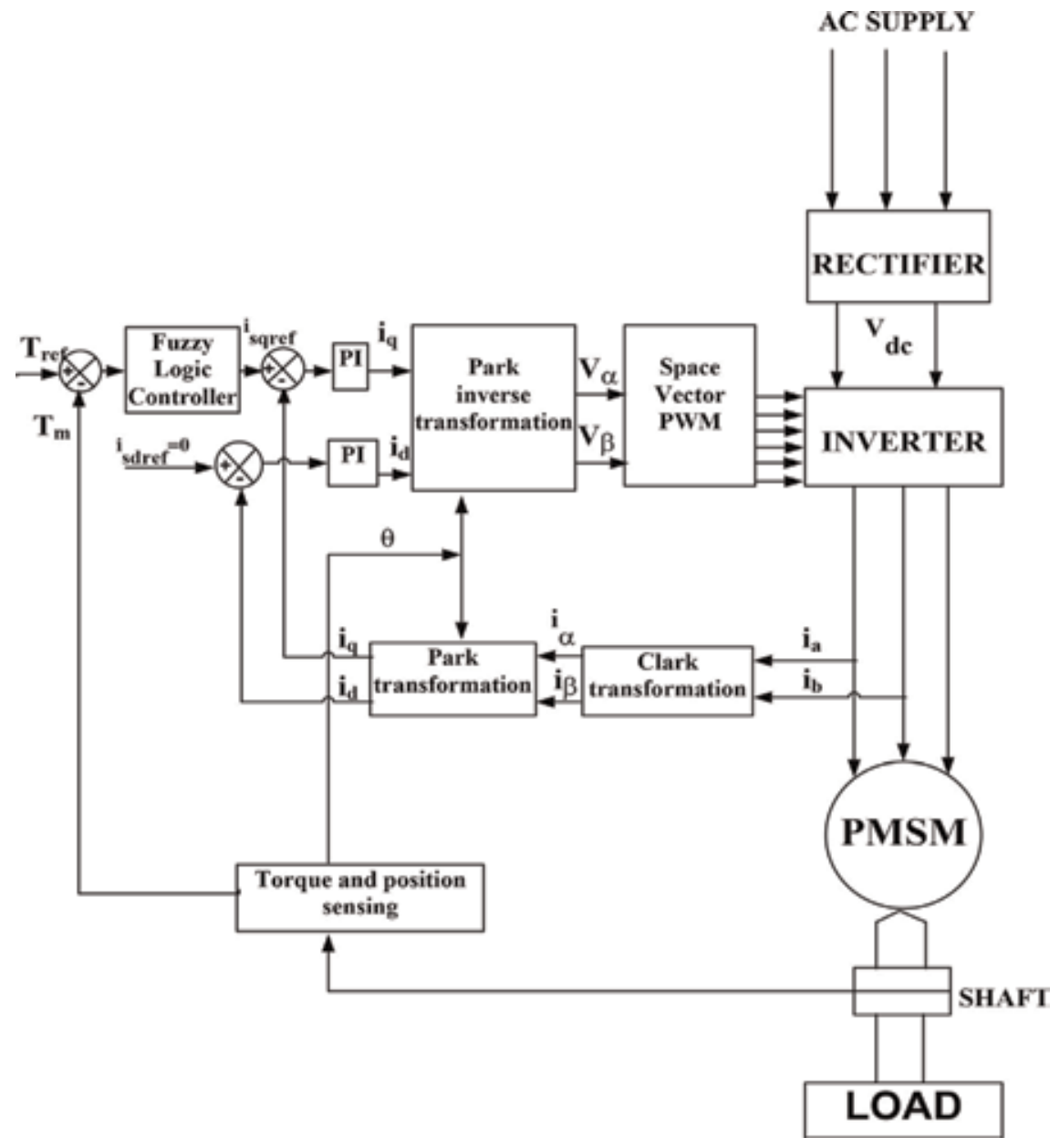

Figure 11.

Block diagram of PMSM driven by FOC using FLC with SVPWM. 


\subsubsection{Space vector pulse width modulation}

Space vector PWM (SVPWM) refers to a special technique of determining the switching sequence of the upper three power transistors of a three-phase voltage source inverter (VSI). There are eight possible combinations of on and off states for the three upper power transistors which determine eight phase voltage configurations. This PWM technique controls the motor based on the switching of space voltage vectors, by which an approximate circular rotary magnetic field is obtained. It approximates the reference voltage Vref by a combination of the eight switching patterns $\left(\mathrm{V}_{0}-\mathrm{V}_{7}\right)$. The vectors $\left(\mathrm{V}_{1}-\mathrm{V}_{6}\right)$ divide the plane into six sectors (each sector: $60^{\circ}$ ). Vref is generated by two adjacent non-zero vectors and two zero vectors. The switching sector is shown in Figure 12 and Table 1 shows the switching vector for inverter.

\subsubsection{PI controller tuning}

The current loop PI controllers compare the actual current with reference current and produce iq and id current, respectively. PI tuning is done by trial and error method.

\subsubsection{Fuzzy logic controller}

The basic concept behind FLC is to utilize the expert knowledge and experience of a human operator for designing a controller an application process whose inputoutput relationship is given by a collection of fuzzy control rules using linguistic variables instead of a complicated dynamic model.

The FLC initially converts the crisp error and change in error variables into fuzzy variables and then are mapped into linguistic labels. Membership functions are associated with each label as shown in which consists of two inputs and one output. The inputs are Torque error and change in torque error and the output is torque limit. Fuzzy Inference System uses "IF... THEN..." statements, and the

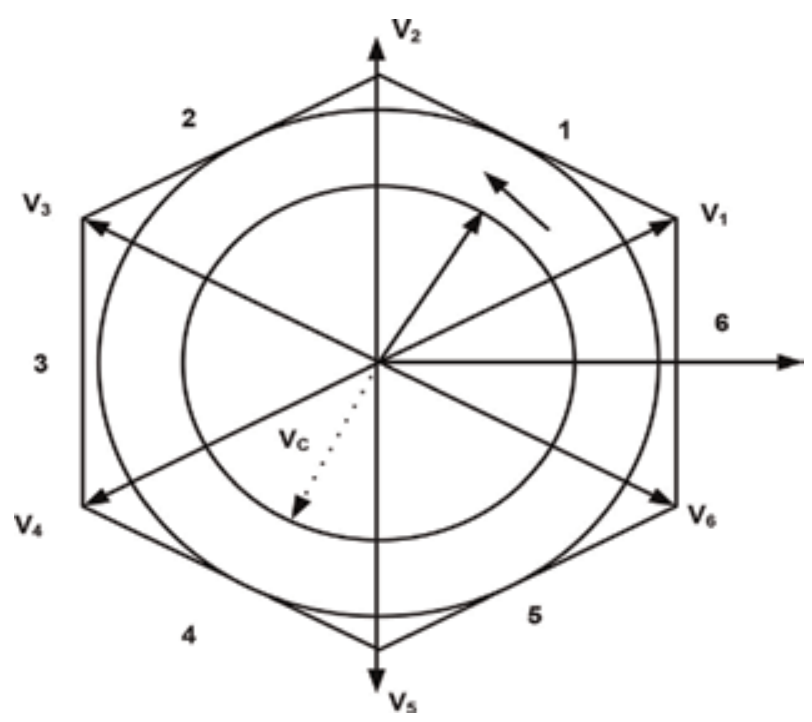

Figure 12.

Switching vectors and sectors. 


\begin{tabular}{lccccccccc}
\hline Vector & A + & B + & C + & A- & B- & C- & VAB & VBC & VCA \\
\hline V0 $=\{000\}$ & OFF & OFF & OFF & ON & ON & ON & 0 & 0 & 0 \\
\hline $\mathrm{V} 1=\{100\}$ & ON & OFF & OFF & OFF & ON & ON & + Vdc & 0 & $-\mathrm{Vdc}$ \\
\hline $\mathrm{V} 2=\{110\}$ & ON & ON & OFF & OFF & OFF & ON & 0 & $+\mathrm{Vdc}$ & $-\mathrm{Vdc}$ \\
\hline $\mathrm{V} 3=\{010\}$ & OFF & ON & OFF & ON & OFF & ON & $-\mathrm{Vdc}$ & $+\mathrm{Vdc}$ & 0 \\
\hline $\mathrm{V} 4=\{011\}$ & OFF & ON & ON & ON & OFF & OFF & $-\mathrm{Vdc}$ & 0 & $+\mathrm{Vdc}$ \\
\hline $\mathrm{V} 5=\{001\}$ & OFF & OFF & ON & ON & ON & OFF & 0 & $-\mathrm{Vdc}$ & $+\mathrm{Vdc}$ \\
\hline $\mathrm{V} 6=\{101\}$ & ON & OFF & ON & OFF & ON & OFF & $+\mathrm{Vdc}$ & $-\mathrm{Vdc}$ & 0 \\
\hline $\mathrm{V} 7=\{111\}$ & ON & ON & ON & OFF & OFF & OFF & 0 & 0 & 0 \\
\hline
\end{tabular}

Table 1.

Switching vectors for inverter.

connectors present in the rule statement are "OR" or "AND" to make the necessary decision rules.

The linguistic labels are divided into seven groups. They are: NB—negative big; NM-negative medium; NS—negative small; Z_zero; PS—positive small; PMpositive medium; PB-positive big. Each of the inputs and the output contain membership functions with all these seven linguistics.

Figure 13 shows the speed error, Figure 14 shows the change in speed error and Figure 15 shows the torque limit.

The mapping of the fuzzy inputs into the required output is derived with the help of a rule base as given in Table 2 .

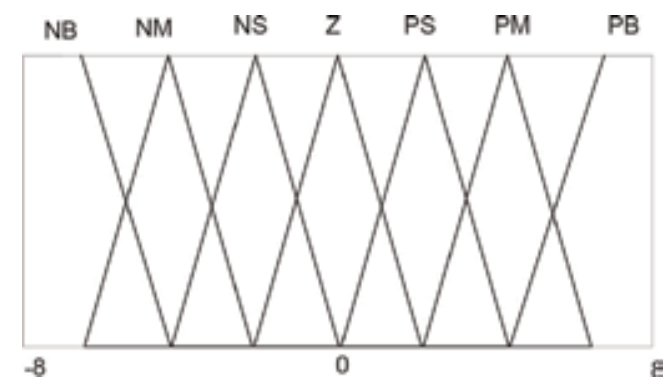

Figure 13.

Torque error input to FLC.

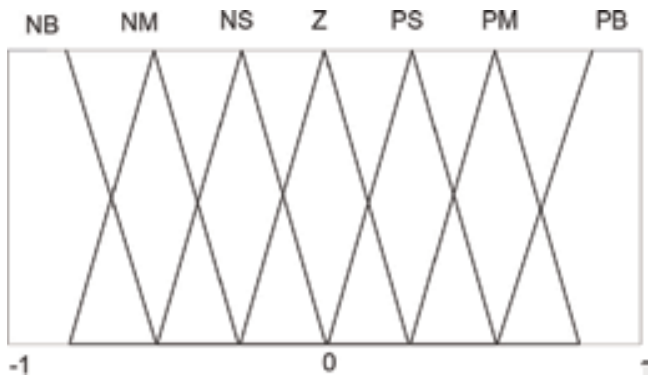

Figure 14.

Change in torque error input to FLC. 


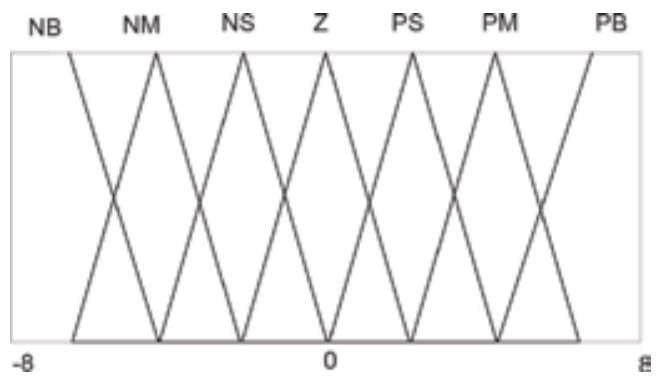

Figure 15.

Torque limit output of FLC.

\begin{tabular}{llllllll}
\hline $\mathbf{e} \boldsymbol{\Delta} \mathbf{e}$ & NB & NM & NS & $\mathbf{Z}$ & PS & PM & PB \\
\hline NB & NB & NB & NB & NM & NM & NS & Z \\
\hline NM & NB & NB & NB & NM & NS & Z & PS \\
\hline NS & NB & NM & NS & NS & Z & PS & PM \\
\hline$Z$ & NM & NM & NS & Z & PS & PM & PM \\
\hline PS & NM & NS & Z & PS & PS & PM & PB \\
\hline PM & NS & Z & PS & PM & PM & PB & PB \\
\hline PB & Z & PS & PM & PM & PB & PB & PB \\
\hline
\end{tabular}

Table 2.

Rules for FLC.

\subsubsection{Simulation results and discussion}

The simulink model of FLC with SVPWM based FOC of PMSM is shown in Figure 16 and that of SVPWM pulse production is shown in Figure 17.

The FOC of PMSM is done using conventional PI controller and FLC using SVPWM techniques using MATLAB version R2009a and the results are compared with other reported results in Table 3. The parameters of PMSM used in the simulation are given in the appendix.

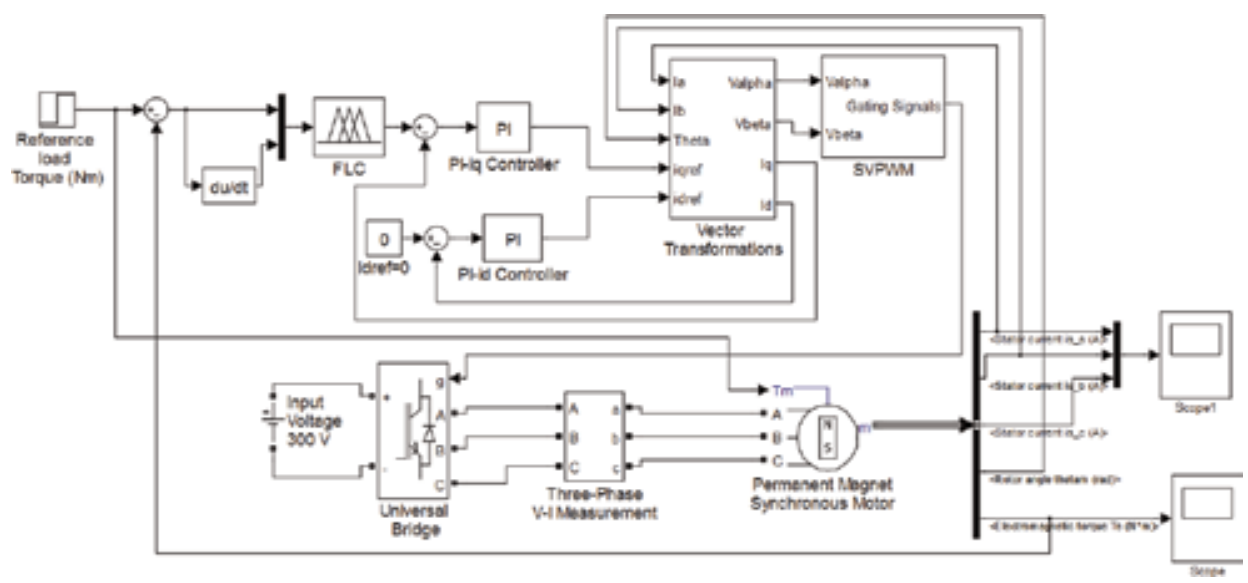

Figure 16.

Simulink model of FLC with SVPWM based FOC of PMSM. 


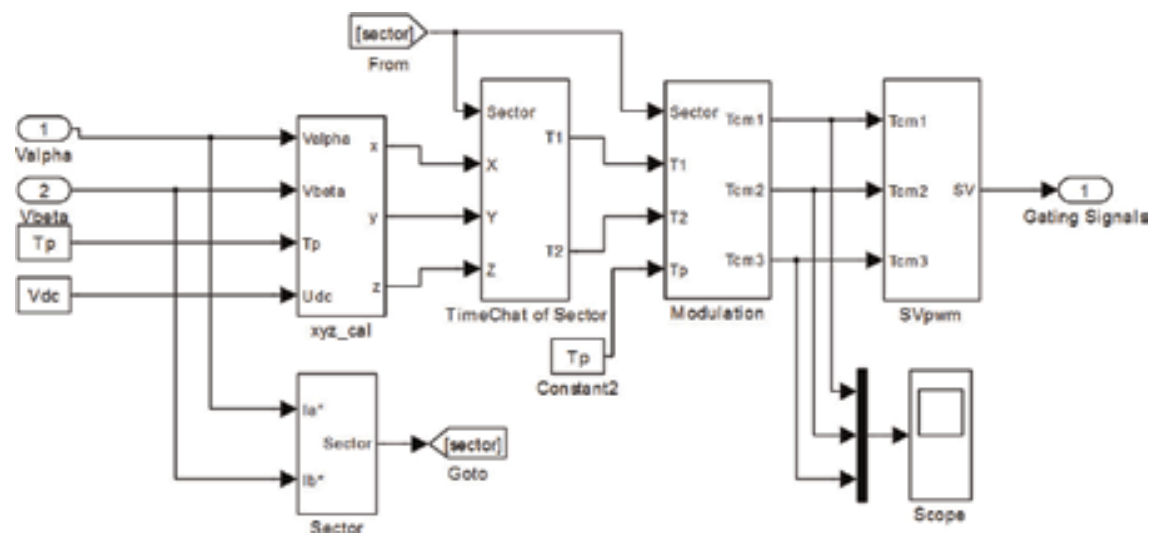

Figure 17.

Simulink model of SVPWM pulse production.

\begin{tabular}{lc}
\hline Control strategies & Torque ripple factor (\%) \\
\hline Proposed FLC with SVPWM & 1.75 \\
\hline Mattavelli et al. [8] & 3.8 \\
\hline Qian et al. [9] & 3.9 \\
\hline Tarnik and Murgas [10] & 4 \\
\hline Hasanien [11] & 12 \\
\hline
\end{tabular}

Table 3.

Comparison of control strategies in PMSM.

The torque ripple can be calculated by using the relation.

$$
\text { Torque ripple factor }=\frac{\text { Peak to peak torque }}{\text { Rated torque }}
$$

The simulation results are shown in Figures 18-20.

From Figure 19 (Torque waveform) it is inferred that the torque ripples oscillates from $7.91 \mathrm{Nm}$ (minimum) to $8.05 \mathrm{Nm}$ (maximum) for the given reference torque of $8 \mathrm{Nm}$.

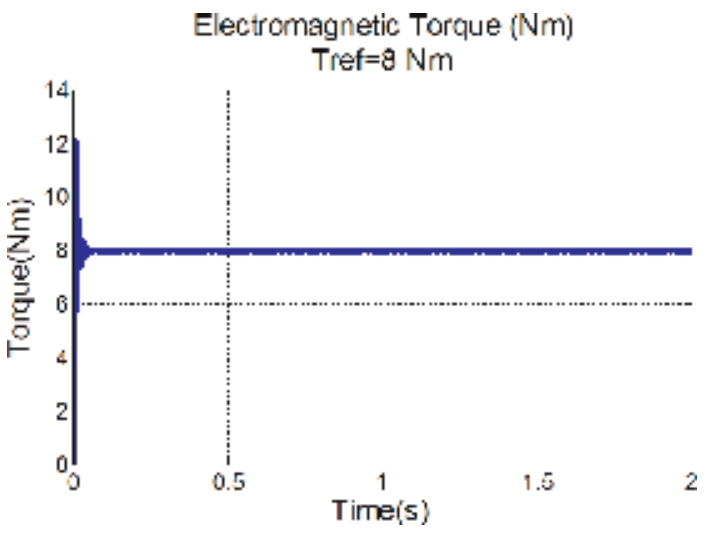

Figure 18.

Torque output for FOC based PMSM using FLC and SVPWM. 


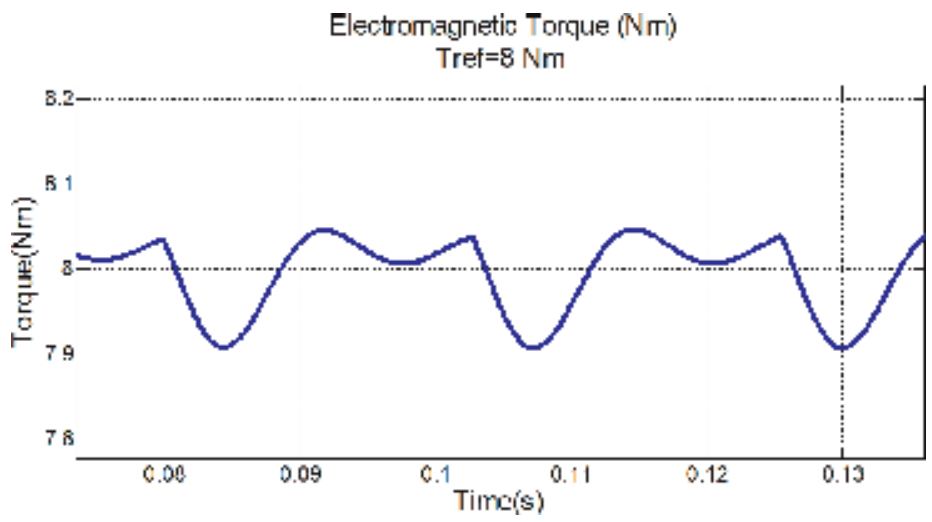

Figure 19.

Torque ripples in FOC based PMSM using FLC and SVPWM.

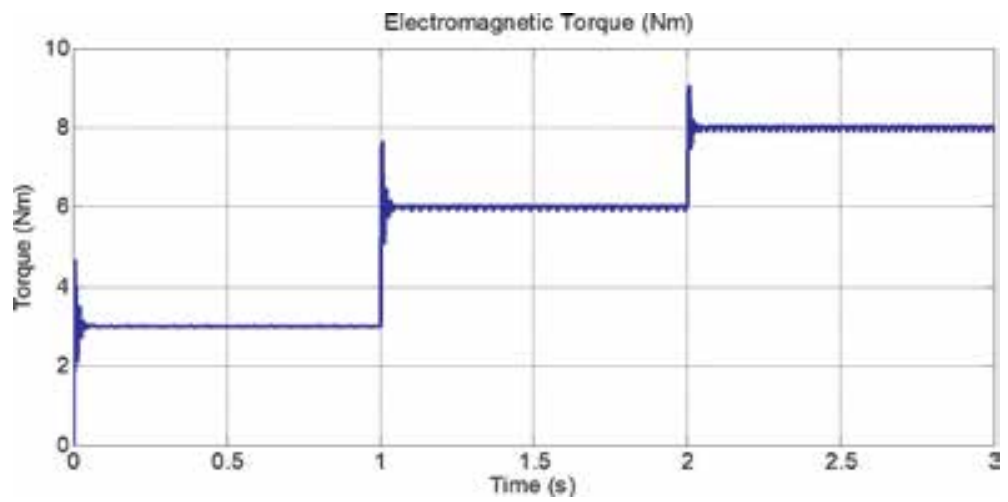

Figure 20.

Dynamic torque using FLC with SVPWM based field controlled PMSM.

Torque ripple factor $(\%)$ as per Eq. 23 is given by $=((8.05-7.91) / 8) \times$ $100=0.14 / 8 \times 100=\mathbf{1} .75$.

It is clear that variation in torque shown in Table 3 is less in case of fuzzy logic controllers and they can achieve a minimum torque ripple than other control techniques. It has been viewed that the discussed control strategy has helped in reducing the torque ripples to $1.75 \%$. Thus by using FLC based controller, ripples are reduced completely.

\section{Advanced topics in PMSM drives}

\subsection{PMSM control with rotor position sensors}

The encoders, resolvers, eddy current sensors are used for rotor position sensing of PMSM control. Absolute encoder has the advantages that it could retain the position information in power outage conditions and for long inactive periods of devices. It is suitable for the applications such as flow control, crane movement, and astronomical telescopes. The position resolvers are the rotary transformers where primary winding is placed on rotor. The induced voltage at secondary winding is shifted by 90 would be different which is based on the rotor shaft angle [12]. 
The PMSM control with speed, torque controller, where rotor angular position sensor is used to get the feedback. The general block diagram is given in Figure 21.

The PMSM control with speed, torque controller, where rotor angular position sensor is used to get the feedback. The general block diagram is given in Figure 22.

\subsection{PMSM control without position sensors}

The control scheme for sensorless PMSM where the rotor position information is used as feedback to controllers is given in Figure 23.

The estimation of stator flux is used to find the stator current at a predicted rotor position. The error in the predicted rotor position is corrected by finding the difference between the estimated stator current and measured current [13].

\subsubsection{Sensorless control schemes for PMSM}

The difficulties in estimating the rotor position due the reasons: 1 . scalar speed estimation and 2. initial position of rotor is not known. The non-measurable variables of PMSM are estimated by the observers. For zero-speed application, salience tracking technique is considered as appropriate, where back-Emf technique fails at low speed. The methods generally used to estimate the rotor position are tracking observer, tracking state filter and arctangent calculation [14].

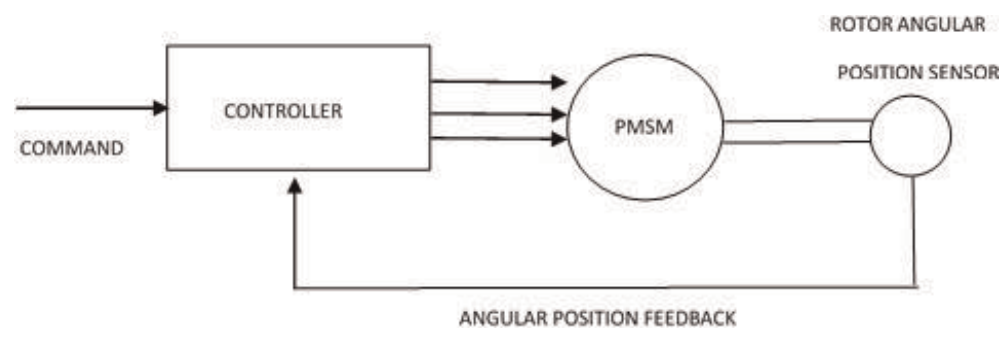

Figure 21.

The general block diagram for PMSM control with rotor position sensor.

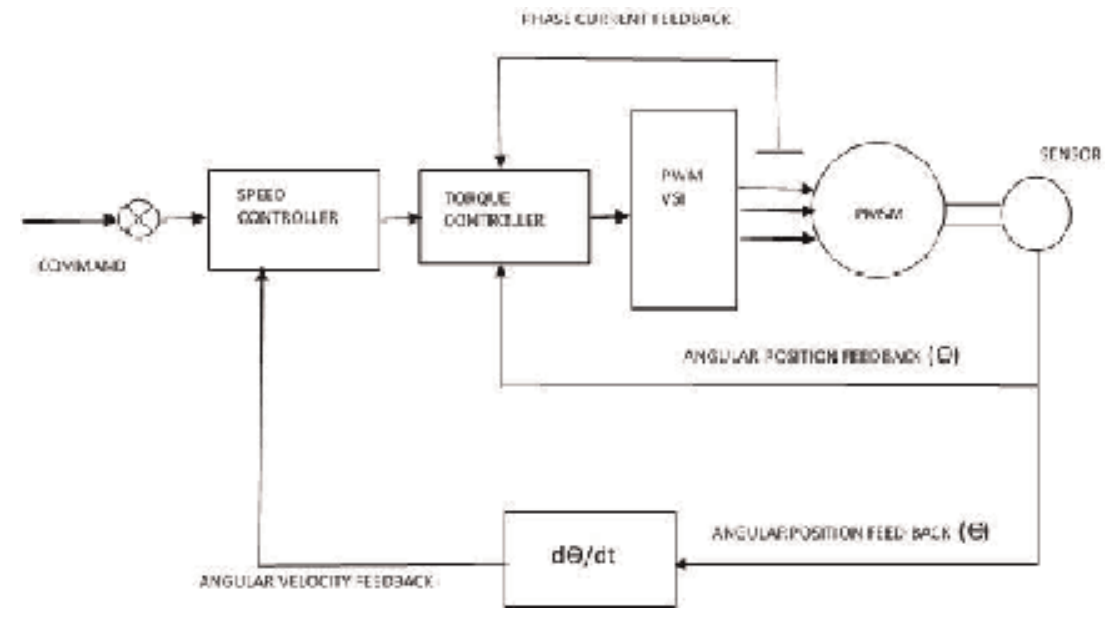

Figure 22.

PMSM control scheme with angular position sensor [13]. 


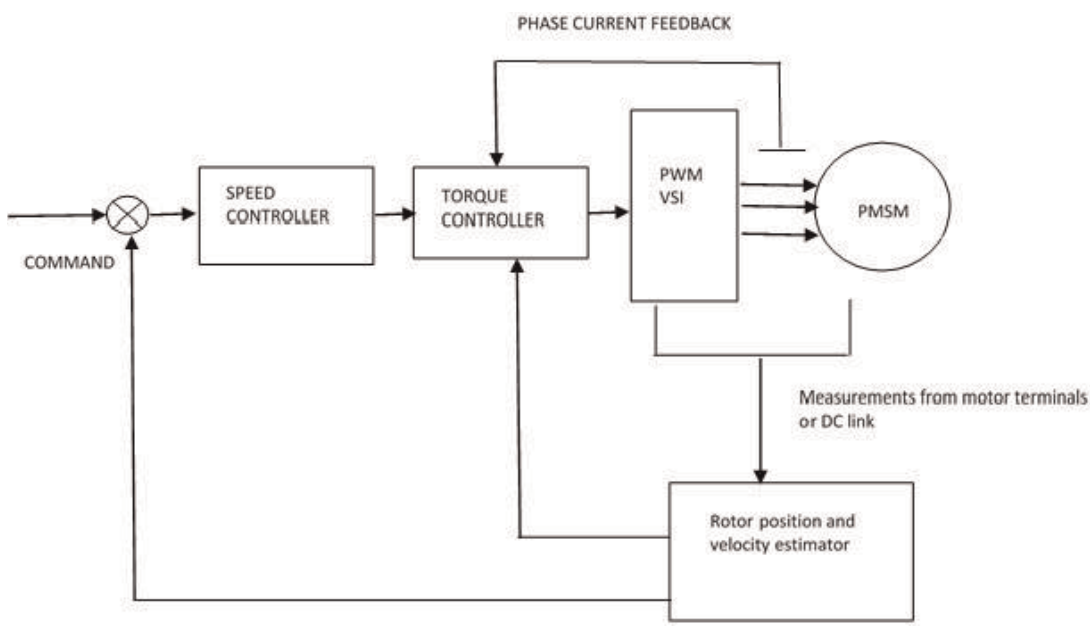

Figure 23.

Sensorless control scheme for PMSM.

The sliding mode observer having sigmoid switching function, effectively suppressed the oscillation of system. The cut-off frequency of LPF is tuned to rotor speed, so that the rotor speed becomes real-time and tunable [15].

In PMSM vector control system with space vector PWM voltage source inverter (SVPVM-VSI), the single shunt current sensing with model reference adaptive system (MRAS) for rotor position sensorless control method is implemented [16].

To estimate the position variable of PMSM: 1. Linear Luenberger observer is constructed 2. PI-type controller which is based on LMI is suggested [17].

To estimate the rotor position: 1 . The delta-sigma modulation 2. CIC extraction filter method are used. It is found that signal-to-noise ratio is high in this technique [18].

The neural network observer used for estimating speed/position of rotor in PMSM, where the observer can do the tracking of the rotor speed at low speed range with greater accuracy [19].

A linearized IPMSM model which is using FOC is created. The rotor speed and phase current of the model is estimated by extended Kalman filter (EKF) [20].

A novel SMO is proposed to achieve greater degree of accuracy in estimating the position and speed of PMSM. Further, the observer reduced the filter links and phase compensation links that achieved a simplification in traditional SMO [21].

A simplified vector control model of PMSM with NNPID controller is created. Automatic tuning of variables could be achieved by using BP NNPID. The overswing phenomenon is eliminated to the maximum extent possible [22].

The feasibility of feedback linearization of PMSM model is shown by MIMO nonlinear system example and then it was combined with vector control method. For handling the nonlinear system, it is showed that feedback linearization is a better method and has better control capability [23].

To estimate the initial position of rotor, a start-up strategy is proposed to overcome the drawback of back emf based control at zero and low speed ranges. The NN based controller is employed to control the current and speed of back emf based FOC of PMSM. The dynamic response of surface mounted type PMSM is improved [24].

A model of IPMSM is developed based on intelligent control method maximum torque per ampere (MTPA) principle in order to achieve the dynamic response characteristics for high precision position servo systems. The shakeless fuzzy controller is proposed with a new control method which is based on single neuron that tunes the output scaling factor of the controller [25]. 
To estimate the rotor position, speed and stator resistance, an interconnected higher order sliding mode observer (HOSMO) for IPMSM is developed [26].

The traditional backstepping control is improved by adding integral control at each step of the tracking and regulation strategies. This permits to reject uncertainties and disturbances.

The stator resistance is influenced by temperature variation that varies randomly. An improved method based on variable parameter PI to compensate the stator resistance is proposed. By constructing the stator flux observer mathematical model of direct torque control (DTC), the problem of error existing in stator flux is solved [27].

\subsubsection{Speed-torque ripple-suppression}

An ILC controller is implemented along with a PI speed controller to minimize the periodic ripples in speed. When the learning algorithm is implemented in frequency domain, it showed better performance in minimizing the ripples in speed than time domain implementation. This is due to the elimination of forgetting factor (FF), in time domain learning method [28].

A new novel method "Intelligent sensor bearing" is implemented by modifying the feedback information that comes from sensor. An Iterative Learning Control (ILC) is used to deal with the periodical errors for reducing ripples in speed [29].

A novel technique "Instantaneous torque control" based on fuzzy logic controller (FLC) with SVPWM is proposed to reduce the torque ripples in PMSM. The simulation results are presented in Section 5.2.3 [30].

To minimize the torque ripples of PMSM, the instantaneous field oriented torque control schemes (a) ILC with hysteresis pulse width modulation (HPWM), (b) ILC with SVPWM are proposed. The simulation results showed that ILC with SVPWM has better control over torque ripples [31].

The speed torque ripple minimization of PMSM is achieved by using neural networks (NN). The conventional PI-controller based vector control method was compared with feed forward NN (FFNN). The simulation showed that NN controller has better control than PI controller [32].

\subsubsection{Application specific PMSM controls}

In high speed traction applications, the motor speed range of IPMSM is extended by single current regulator flux-weakening control strategy which results in steady operation of motor with high speed [29].

\section{Acknowledgements}

We thankful to the management and School of Electrical and Mechanical Engineering of Addiss Ababa Science and Technology University, Addiss Ababa, Ethiopia for their encouragement, support and facilities provided for our book chapter work.

\section{Nomenclature}

$\begin{array}{ll}\mathrm{f}_{\mathrm{c}} & \text { crossover frequency } \\ \mathrm{i}_{\mathrm{d}} & \mathrm{d} \text {-axis current } \\ \mathrm{L}_{\mathrm{dm}} & \mathrm{d} \text {-axis magnetizing inductance }\end{array}$




$\begin{array}{ll}\mathrm{L}_{\mathrm{d}} & \text { d-axis self-inductance } \\ \mathrm{V}_{\mathrm{d}} & \text { d-axis voltage } \\ \rho & \text { derivative operator } \\ \mathrm{T}_{\mathrm{e}} & \text { develop electromagnetic torque } \\ \mathrm{d} & \text { direct or polar axis } \\ \mathrm{DTC} & \text { direct torque control } \\ \omega_{\mathrm{r}} & \text { electrical speed } \\ \mathrm{i}_{\mathrm{f}} & \text { equivalent permanent magnet field current } \\ \mathrm{L}_{\mathrm{s}} & \text { equivalent self-inductance per phase } \\ \lambda_{\mathrm{d}} & \text { flux linkage due d axis } \\ \lambda_{\mathrm{q}} & \text { flux linkage due q axis } \\ \lambda_{\mathrm{dm}} & \text { flux linkage due to rotor magnets linking the stator } \\ \mathrm{B} & \text { friction } \\ \mathrm{FLC} & \text { fuzzy logic controller } \\ \mathrm{J} & \text { inertia } \\ \mathrm{k}_{\mathrm{i}} & \text { integral control gain } \\ \mathrm{T}_{\mathrm{L}} & \text { load torque } \\ \omega_{\mathrm{rated}} & \text { motor rated speed } \\ \mathrm{T}_{\mathrm{m}} & \text { motor torque } \\ \mathrm{P} & \text { number of poles } \\ \mathrm{I}_{\mathrm{m}} & \text { peak value of supply current } \\ \lambda_{\mathrm{f}} & \text { PM flux linkage or field flux linkage } \\ \mathrm{k}_{\mathrm{p}} & \text { proportional control gain } \\ \mathrm{i}_{\mathrm{q}} & \text { q-axis current } \\ \mathrm{L}_{\mathrm{qm}} & \text { q-axis magnetizing inductance } \\ \mathrm{L}_{\mathrm{q}} & \text { q-axis self-inductance } \\ \mathrm{V}_{\mathrm{q}} & \text { q-axis voltage } \\ \mathrm{q} & \text { quadrature or interpolar axis } \\ \mathrm{T}_{\mathrm{ref}} & \text { reference motor torque } \\ \theta_{\mathrm{r}} & \text { rotor position } \\ \omega_{\mathrm{m}} & \text { rotor speed } \\ \mathrm{L}_{\mathrm{m}} & \text { self-inductance } \\ \mathrm{L}_{\mathrm{ls}} & \text { stator leakage inductance } \\ \mathrm{R}_{\mathrm{s}} & \text { stator resistance } \\ \mathrm{i}_{\mathrm{a}}, \mathrm{i}_{\mathrm{b}}, \mathrm{i}_{\mathrm{c}} & \text { three phase currents } \\ \mathrm{V}_{\mathrm{a}}, \mathrm{V}_{\mathrm{b}}, \mathrm{V}_{\mathrm{c}} & \text { three phase voltage } \\ & \end{array}$

\section{Appendix 1: parameters of PMSM}

\begin{tabular}{lc}
\hline Rated power & $1 \mathrm{HP}$ \\
\hline Rated torque & $8 \mathrm{Nm}$ \\
\hline Rated voltage & $300 \mathrm{~V}$ \\
\hline Rated current & $2.5 \mathrm{~A}$ \\
\hline Stator resistance & $0.9585 \Omega$ \\
\hline Inductance $\mathrm{L}_{\mathrm{d}}$ & $0.00525 \mathrm{H}$ \\
\hline Inductance $\mathrm{L}_{\mathrm{q}}$ & $0.00525 \mathrm{H}$ \\
\hline Magnetic flux & 0.1827 weber \\
\hline No. of poles & 8 \\
\hline
\end{tabular}




\begin{tabular}{lc}
\hline Moment of inertia & $0.0006329 \mathrm{~kg} \mathrm{~m}^{2}$ \\
\hline Friction factor & $0.0003035 \mathrm{Nm} /(\mathrm{rad} / \mathrm{s})$ \\
\hline
\end{tabular}

\section{Appendix 2: block parameters}

\begin{tabular}{cc}
\hline $\mathrm{kp}$ & 10 \\
\hline $\mathrm{ki}$ & 1 \\
\hline
\end{tabular}

\section{Author details}

Adhavan Balashanmugham ${ }^{1 *}$ and Mockaisamy Maheswaran ${ }^{2}$

1 Department of Electrical and Computer Engineering, Addis Ababa Science and Technology University, Addis Ababa, Ethiopia

2 Department of Electromechanical Engineering, Addis Ababa Science and Technology University, Addis Ababa, Ethiopia

*Address all correspondence to: adhav14@gmail.com

\section{IntechOpen}

(C) 2019 The Author(s). Licensee IntechOpen. This chapter is distributed under the terms of the Creative Commons Attribution License (http://creativecommons.org/licenses/ by/3.0), which permits unrestricted use, distribution, and reproduction in any medium, provided the original work is properly cited. (cc) BY 


\section{References}

[1] Bimbra PS. Electric Machinery. 7th ed. Delhi, India: Khanna Publishers; 2011. p. 1060. ISBN: 978-8174091734

[2] Bose BK. Power Electronics and Variable Frequency Drives. 1st ed. NJ, USA: Wiley John \& Sons; 1996. p. 660. ISBN: 978-0-780-31084-1

[3] Krishnan R. Electric Motor Drives Modeling, Analysis, and Control. NJ, USA: Prentice Hall; 2011. p. 652. ISBN: 9780130910147

[4] Bose BK. Modern Power Electronics and AC Drives. NJ, USA: Prentice Hall; 2002. p. 738. ISBN: 9780130167436

[5] Pillay P, Krishnan R. Modeling of permanent magnet motor drives. IEEE Transactions on Industrial Electronics. 1988;354:537-541. DOI: 0278-0046/88/ 1100-0537

[6] The MathWorks Incorporation. SimPowerSystems ${ }^{\mathrm{TM}}$ User's Guide [Internet]. 2013. Available from: http:// www.mathworks.in/help/pdf_doc/ph ysmod/powersys/powersys.pdf [Accessed: 10 June 2019]

[7] Novotny DW, Lipo TA. Vector Control and Dynamics of AC Drives. New York, USA: Oxford University Press; 1998. p. 456. ISBN: 9780198564393

[8] Mattavelli P, Tubiana L, Zigliotto M. Torque-ripple reduction in PM synchronous motor drives using repetitive current control. IEEE Transactions on Power Electronics. 2005:1423-1431

[9] Qian W, Panda SK, Xu JX. Torque ripple minimization in PM synchronous motors using iterative learning control. IEEE Transactions on Power Electronics. 2004:272-279

[10] Tarnik M, Murgas J. Additional adaptive controller for mutual torque ripple minimization in PMSM drive systems. In: Ni Y-Q, Ye X-W, editors. Proceedings of 18th IFAC World Congress. Milano, Italy; 2011. pp. $4119-4124$

[11] Hasanien H. Torque ripple minimization of permanent magnet synchronous motor using digital observer controller. Energy Conversion and Management. 2010:98-104

[12] Kiran N, Anitha Nair AS, Sri

Lakshmi D. Permanent magnet synchronous motor control with speed feedback using a resolver. International Journal of Advanced Research in Education and Technology (IJARET). 2016;3(4):74-78

[13] Chandana Perera PD. Sensorless control of permanent-magnet synchronous motor drives [thesis]. Denmark: Aalborg University; 2002

[14] Glumineau A, de León Morales J. Sensorless AC Electric Motor Control Robust Advanced Design Techniques and Applications. Switzerland: Springer International Publishing; 2015. pp. 121-137. DOI: 10.1007/978-3319-14586-0-4

[15] Hu W, editor. Electronics and Signal Processing. Berlin, Heidelberg:

Springer-Verlag; 2011. pp. 533-538. DOI: 10.1007/978-3-642-21697-8

[16] Ma H, Wang W, editors. Mechatronics and Automatic Control Systems. Heidelberg, New York, Dordrecht, London: Springer Cham; 2014. pp. 727-732. DOI: $10.1007 / 978-3-$ 319-01273-5

[17] Sun X, Yi Y, Cao S, Liu H, Zhang T. Robust PI-type position controller design for permanent magnet synchronous motor using LMI techniques. In: Deng Z, Li H, editors. 
Proceedings of the 2015 Chinese Intelligent Automation Conference. Berlin, Heidelberg: Springer-Verlag; 2015. pp. 405-410. DOI: $10.1007 / 978-3-$ 662-46463-2

[18] Zhu B, Zhao H, Zhu J, He Y. Design and implementation of a PMSM rotor position detecting system with high speed and high precision. In: Deng Z, $\mathrm{Li} \mathrm{H}$, editors. Proceedings of the 2015 Chinese Intelligent Automation Conference. Berlin, Heidelberg: Springer-Verlag; 2015. pp. 513-518. DOI: 10.1007/978-3-662-46463-2

[19] Shoeb Hussain, Mohammad Abid Bazaz. Sensorless control of PMSM drive with neural network observer using a modified SVPWM strategy. In: Juan Manuel Corchado Rodriguez, Sushmita Mitra, Sabu M. Thampi, El-Sayed ElAlfy, editors. Intelligent Systems Technologies and Applications. Springer International Publishing AG; 2016. p. 857-862. DOI 10.1007/978-3319-47952-1.

[20] Mathianantham L, Gomathi V, Ramkumar K, Balasubramanian G. State estimation of interior permanent magnet synchronous motor drives using EKF. In: Suresh LP, Panigrahi BK, editors. Proceedings of the International Conference on Soft Computing Systems. India: Springer; 2016. pp. 719-724. DOI: 10.1007/978-81-322-2671-0

[21] Zhu X. Research on sensorless control of PMSM based on a novel sliding mode observer. In: Deng Z, editor. Proceedings of 2017 Chinese Intelligent Automation Conference. Singapore Pte Ltd.: Springer Nature; 2108. pp. 193-198. DOI: $10.1007 /$ 978-981-10-6445-6

[22] Zhang L, Xu B-J, Wen K-L, Li Y-H. The study of permanent magnetic synchronous motor control system through the combination of BP neural network and PID control. In: Juang J, Huang Y-C, editors. Intelligent
Technologies and Engineering Systems. Heidelberg, New York, Dordrecht, London: Springer; 2013. pp. 311-316. DOI: 10.1007/978-1-4614-6747-2

[23] Wang H, Liu X. Permanent magnet synchronous motor, feedback linearization vector control. In: $\mathrm{Ma} \mathrm{H}$, Wang W, editors. Mechatronics and Automatic Control Systems. Cham, Heidelberg, New York, Dordrecht, London: Springer; 2014. pp. 601-606. DOI: 10.1007/978-3-319-01273-5

[24] Nagarajan VS, Subashini TS. Backemf-based sensorless field oriented control of PMSM using neural-networkbased controller with a start-up strategy. In: Suresh LP, Dash SS, Panigrahi BK, editors. Artificial Intelligence and Evolutionary Algorithms in Engineering Systems. India: Springer; 2015. pp. 449-454. DOI: $10.1007 / 978-81-$ 322-2135-7

[25] Shao M, Yu H, Wang Z, Xie H, Zhao S. Position control of interior permanent synchronous motor based on improved shakeless fuzzy controller. In: Deng Z, Li H, editors. Proceedings of the 2015 Chinese Intelligent Automation Conference. Berlin, Heidelberg: Springer-Verlag; 2015. pp. 83-88. DOI: 10.1007/978-3-662-46463-2

[26] Glumineau A, de Leon Morales J. Sensorless AC Electric Motor Control Robust Advanced Design Techniques and Applications. Switzerland: Springer International Publishing; 2015. pp. 112-117. DOI: 10.1007/978-3319-14586-0-4

[27] Zhang S, Cui W, Shen Y, Liu H. Direct torque control of permanent magnet synchronous motor at low speed using a variable PI feedback flux observer. In: Huang B, Yao Y, editors. Proceedings of the 5th International Conference on Electrical Engineering and Automatic Control. Berlin, Heidelberg: Springer-Verlag; 2016. 
pp. 927-932. DOI: 10.1007/978-3-

662-48768-6

[28] Xu J-X, Panda SK, Lee TH, editors.

Real-Time Iterative Learning Control

Design and Applications. London:

Springer-Verlag; 2009. pp. 101-106.

DOI: $10.1007 / 978-1-84882-175-0$.

[29] Hu T, Lin F, Cui L, Yuan Q, Yang Z. The flux-weakening control of interior permanent magnet synchronous traction motors for high-speed train. In: Ni Y-Q, Ye X-W, editors. Proceedings of the 1st International Workshop on High-Speed and Intercity Railways. Berlin, Heidelberg: Springer-Verlag; 2012. pp. 451-461. DOI: $10.1007 / 978-3-$ 642-27960-7

[30] Adhavan B, Birundha MS, Ravichandran CS, Jagannathan V. Torque ripple reduction in PMSM using FLC. Australian Journal of Basic and Applied Sciences. 2013;61(2):61-68. ISSN 1991-8178

[31] Adhavan B, Jagannathan V. Performance comparison of hysteresis pulse width modulation and space vector pulse width modulation technique for torque ripple reduction in permanent magnet synchronous motor using iterative learning control. Journal of Vibration and Control. 2012;20(5):

698-712. DOI: $10.1177 /$

1077546312463748

[32] Adhavan B, Jagannathan V. Torque ripple minimization in permanent synchronous motor using neural networks. Advances in Modelling and Analysis C Automatic Control. 2013;68 (2):65-80. ISSN: 1240-4535 



\title{
Investigation on Internal Short Circuit Identification of Lithium- Ion Battery Based on Mean- Difference Model and Recursive Least Square Algorithm
}

\author{
Xu Zhang, Yue Pan, Enhua Wang, Minggao Ouyang, \\ Languang Lu, Xuebing Han, Guoqing Jin, Anjian Zhou \\ and Huiqian Yang
}

\begin{abstract}
Electric vehicles powered by lithium-ion batteries take advantages for urban transportation. However, the safety of lithium-ion battery needs to be improved. Self-induced internal short circuit of lithium-ion batteries is a serious problem which may cause battery thermal runaway. Accurate and fast identification of internal short circuit is critical, while difficult for lithium-ion battery management system. In this study, the influences of the parameters of significance test on the performance of an algorithm for internal short circuit identification are evaluated experimentally. The designed identification is based on the mean-difference model and the recursive least square algorithm. First, the identification method is presented. Then, two characteristic parameters are determined. Subsequently, the parameters of the significance calculation are optimized based on the measured data. Finally, the effectiveness of the method for the early stage internal short circuit detection is studied by an equivalent experiment. The results indicate that the detection time can be shortened significantly via a proper configuration of the parameters for the significance test.
\end{abstract}

Keywords: lithium-ion battery, internal short circuit identification, significance test, mean-difference model, equivalent experiment

\section{Introduction}

Safety and energy saving as well as environmental protection are the key points for automobile industry development $[1,2]$. Lithium-ion batteries have been widely used due to their high energy density, long cycle life, low environmental pollution, no memory effect, and high charge and discharge rates [3-5]. With the rapid development of materials technology, the energy density of lithium-ion batteries is gradually increased, causing the thermal runaway events accordingly. Its safety 
problem must be taken into account seriously [6-9]. The safety of batteries may affect the confidence of the users of electric vehicles. Therefore, battery safety is one of the biggest obstacles for the application of electric vehicles. Internal short circuit is an important step leading to the thermal runaway. Thus an accurate and rapid identification for internal short circuit is necessary and needs to be solved [10].

The internal short circuit can be detected by comparing the output of the battery with a threshold or a predicted value via a mathematical model. Ikeuchi et al. measured the electricity output of the battery and compared with a threshold computed by the ampere-hour integration [11]. Ouyang et al. proposed three characteristic parameters according to the parameter effect and the depleting effect of an internal short-circuited battery. The variations of the open circuit voltage (OCV) and the internal resistance were determined based on a mean-difference model. Then, a significance criterion and a fault tolerance mechanism were used to judge whether the internal short circuit happened [12]. Feng et al. used a 3D electrochemical-thermal model to simulate the internal short circuit and regarded it as a parameter estimation problem [13]. The measured voltage and the temperature of the cell were input to a model to evaluated excessive depletion of the capacity and abnormal heat generation. The internal short circuit resistance was estimated by Seo et al. according to the SOC variation and the discrete ampere-hour integrals from an equivalent circuit model with internal short circuit branches [14]. Xia et al. employed a voltage correlation coefficient and a threshold to determine whether an internal short circuit occurred via capturing the initial abnormal voltage signal [15]. This method could eliminate the influence of the SOC. Zhang et al. designed a low-pass filter to estimate the leakage current and the resistance of the internal short circuit cell in real time [16]. Kang et al. developed a multi-fault diagnostic strategy based on an interleaved voltage measurement topology. An improved correlation coefficient method was employed to eliminate the inconsistency among the cells and the measurement errors [17]. Kong et al. used a principle of similar charging voltage to obtain the remaining charging electricity and used the average voltage to obtain the internal short circuit resistance and thus the internal short circuit was identified. However, the charging voltage does not have similarities in some cases, and the average voltage may not be correctly obtained [18]. Feng et al. analyzed the characteristics of internal short circuit using an electrochemicalthermal coupling model. This is significant for internal short circuit identification, but it also reveals the difficulty of internal short circuit identification [19].

The operation conditions of the lithium-ion battery in an electric vehicle are very complicated and its safety is critical. Therefore, it is important to find a method which can detect the self-induced internal short circuit in time. Our previous study proposed a method to detect the internal short circuit [12]. However, the detection time of this method is still not satisfactory. In this study, the identification method for the internal short circuit is modified, and the influences of the parameters of the significance test are evaluated. Then, the modified identification algorithm is validated via an equivalent experiment. First, the basic working principle and procedures for the internal short circuit detection are introduced based on the mean-difference model. Then, the parameters for the internal short circuit identification are configured. Next, a replacement experiment is carried out, and the results of this method are computed. Subsequently, the results of a significance test are analyzed. The outcomes indicate that the detection time can be shortened significantly compared with those of the previous study. Finally, the possibility of the identification algorithm for early-stage internal short circuit detection is investigated. The results of this study can provide a reference for the practical applications so that the safety of lithium-ion batteries can be improved. 


\section{Identification method}

The mathematical model of the designed identification method is presented in this section. First, the equivalent circuit model is introduced. Then, the meandifference model and the recursive least square algorithm are described. Finally, the identification procedure is illuminated.

\subsection{Equivalent circuit model}

Figure 1(a) shows the equivalent circuit model of a lithium-ion battery with an internal short circuit resistor, where $R_{I S C r}$ and $I_{I S C r}$ are the resistance and the current of the internal short circuit, respectively:

Based on Figure 1(a), the OCV can be expressed by

$$
U=\frac{R_{I S C r}}{R+R_{I S C r}} E-\frac{R_{I S C r}}{R+R_{I S C r}} R I .
$$

When the internal short circuit resistance approaches infinite, it can be taken as there is no internal short circuit. However, if it is less than a certain value, an internal short circuit branch may be considered to be generated in the battery cell. The parameter effect is the change of internal resistance and open circuit voltage due to the internal short circuit branch according to Eq. (1). The depleting effect is the drop of the open circuit voltage and the fluctuation of the internal resistance caused by the self-discharge of the internal short circuit battery.

\subsection{Mean-difference model}

The parameters of the equivalent circuit model for different cells inside a battery pack are not the same due to the discrepancies of material ingredients, manufacturing processes, and working conditions. The mean-difference model includes a mean model for the pack and a difference model for each cell, which can be used to represent difference among the cells. The parameters of the mean model are obtained according to the average performance of the battery pack. Meanwhile, the difference model displayed in Figure 1(b) describes the difference of a specific cell against the mean model. In Figure 1(b), $\Delta U_{i}, \Delta E_{i}$, and $\Delta R_{i}$ are the differences between the $i$-th cell and the mean model of the battery pack. For a battery pack in series connection, the current flowing through each cell is uniform.

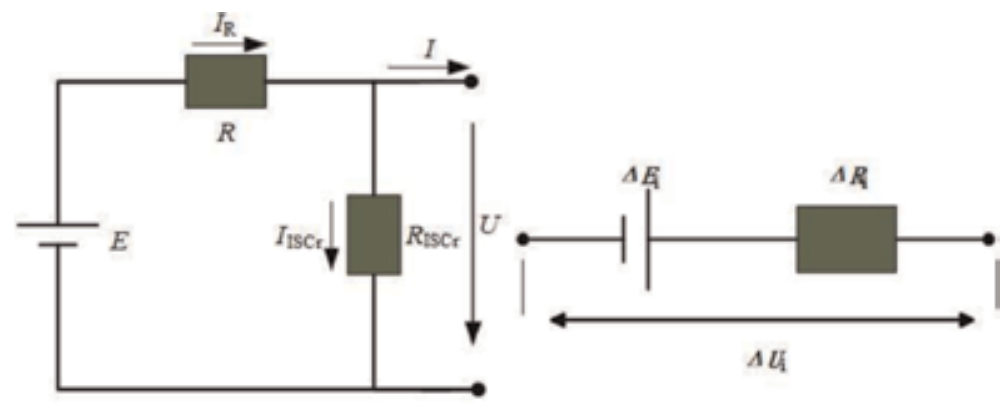

(a)

(b)

Figure 1.

Equivalent circuit of lithium-ion battery: (a) circuit with internal short circuit resistor and $(b)$ mean-difference model of a cell. 
$U_{\text {mean }}$ is the average cell voltage calculated by excluding the highest and lowest voltages among these cells.

\subsection{Recursive least square algorithm}

For a battery pack in series layout, the total current and the terminal voltage can be measured. Accordingly, $U_{\text {mean }}$ and $\Delta U_{i}$ can be calculated by Eqs. (2) and (3):

$$
\begin{gathered}
\Delta U_{i}=\Delta E_{i}-I \Delta R_{i} \\
\Delta U=U_{i}-U_{\text {mean }}
\end{gathered}
$$

After that, $\Delta E_{i}$ and $\Delta R_{i}$ can be identified by the recursive least square (RLS) algorithm with a forgetting factor, which is represented by Eqs. (4)-(7). In this algorithm, $\Delta E_{i}$ and $\Delta R_{i}$ are the parameters needed to be identified, and $\varphi$ is the recursor:

$$
\begin{gathered}
Y=\Delta U_{i} \\
Y=\varphi \bullet \theta \\
\theta=\left(\Delta E_{i}, \Delta R_{i}\right)^{T} \\
\varphi=(1,-I)
\end{gathered}
$$

The calculation process of the RLS algorithm with a forgetting factor can be expressed by Eqs. (8)-(12):

$$
\begin{gathered}
y(k)=\varphi(k) \theta(k)+e(k) \\
e(k)=y(k)-\varphi(k) \hat{\theta}(k-1) \\
K(k)=\frac{P(k-1) \varphi^{T}(k)}{\lambda+\varphi(k) P(k-1) \varphi^{T}(k)} \\
P(k)=\frac{P(k-1)-K(k) \varphi(k) P(k-1)}{\lambda} \\
\hat{\theta}(k)=\hat{\theta}(k-1)+K(k) e(k)
\end{gathered}
$$

where $y(k)$ is the system output, $\varphi(k)$ is the vector that can be measured, $\theta(k)$ is the vector to be estimated, $P(k)$ is the covariance matrix, $K(k)$ is the gain, and $\lambda$ is the forgetting factor.

\subsection{Identification procedure}

The variations of $\Delta E_{i}$ and $\Delta R_{i}$ are due to the parameter effect and the depleting effect as well as the inconsistencies among all the cells of the battery pack. The characteristic parameters that can be used to identify the internal short circuit should reflect the difference between the internal short circuit cell and the others and can be represented by the mean-difference model. At the same time, the battery SOC and the internal short circuit resistance have great influences on the internal short circuit identification [20]. There is a one-to-one correspondence between the SOC and the OCV. As a result, $\Delta E$ can reflect the discharge capacity of the battery, which relates to the situations of the internal electrochemical reaction of the battery. $f l u c(\Delta R)$ is the fluctuation of the computed internal short circuit resistance in a certain interval and can be used to label the event of the internal short circuit of the battery cell. In our previous investigation, three different 
parameters including $\Delta E_{i}, \mathrm{~d}\left(\Delta E_{i}\right) / d t$ and $f l u c\left(\Delta R_{i}\right)$ were used to detect the internal short circuit independently [12]. However, the performance of this method for the early-stage internal short circuit detection was not satisfied. Therefore, a modified method taking into account both $\Delta E_{i}$ and fluc $\left(\Delta R_{i}\right)$ is designed in this study.

The main decision process is described in Figure 2. First, the terminal voltage and the operation current of the $i$-th cell are measured, and the average terminal voltage is calculated. Then, $\Delta U_{i}$ can be determined by the mean-difference model. Next, the parameters $\Delta E_{i}$ and $\Delta R_{i}$ are identified by the RLS method. According to the parameter effect and the depleting effect, the value of $\Delta E_{i}$ of the internal short circuit battery is always negative, while the value of $\Delta R_{i}$ fluctuates up and down. Then, the internal short circuit is judged by a significance calculation. In this study, to improve the accuracy of identification, an anti-false alarm mechanism is adopted; if $80 \%$ of 150 continuous data range of the significance calculation results exceed a prescribed threshold, an event of internal short circuit is setup by the characteristic parameter. Only these two characteristic parameters $\left(\Delta E_{i}\right.$ and fluc $\left.\left(\Delta R_{i}\right)\right)$ detect the event of internal short circuit simultaneously; a final decision of the internal short circuit is confirmed. This anti-false alarm mechanism is a modification based on the previous method in [12].

The forgetting factor $\lambda$ affects the stability of the least squares algorithm. The stability of the algorithm increases as the $\lambda$ approaches 1 . However, the identification precision for the actual parameter variation diminishes. When $\lambda$ decreases, the calculated results may diverge. An optimal range of $\lambda$ is obtained as $0.99-0.995$ based on the test results with different internal short circuit resistances. To keep the error as small as possible, especially for the value of $\Delta R_{i}$, while maintaining the RLS algorithm stable, the forgetting factor is set to 0.992 in this study.

In the previous method [12], $d\left(\Delta E_{i}\right) / d t$ was also used to identify the internal short circuit. In this study, it is found that $d\left(\Delta E_{i}\right) / d t$ is not reliable due to limitations of the sampling frequency and the sampling accuracy and is not recommended. $\Delta E_{i}$ and $\Delta R_{i}$ are directly obtained by the identification algorithm. The fluctuation value fluc $\left(\Delta R_{i}\right)$ of the internal resistance is obtained as the standard deviation of 150 continuous sampling points adjacent to the current moment, which is expressed by

$$
f l u c(\Delta R(k T))=\operatorname{StDev}\{\Delta R((k-149) T), \Delta R((k-148) T), \ldots, \Delta R(k T)\} .
$$

where StDev is the standard deviation. Significance calculation results are obtained according to the extreme values of characteristic parameters, the average value $\mu$, and the standard deviation $\sigma$. The $\mu$ and $\sigma$ are calculated by removing the

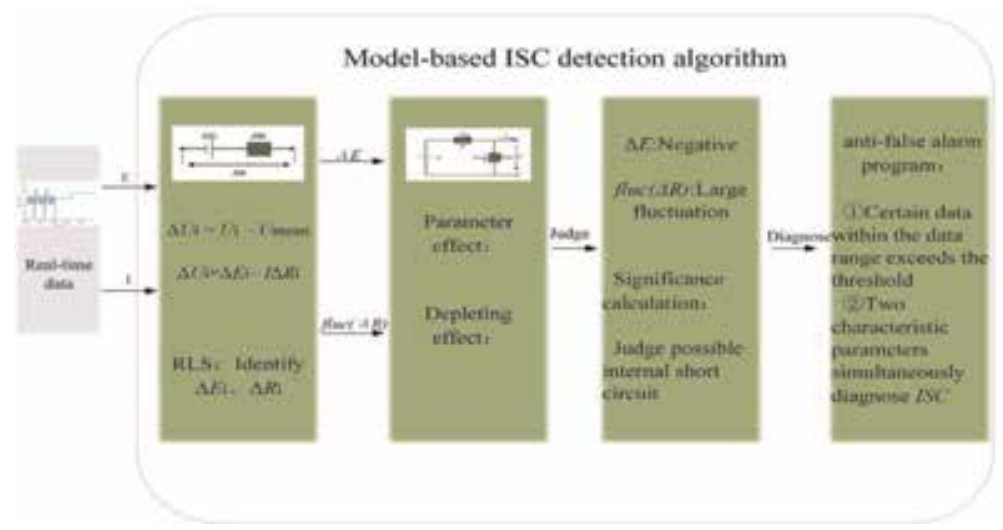

Figure 2.

Detection procedure for internal short circuit. 
extreme values of the characteristic parameters. The positive and negative significances can be represented by

$$
\begin{aligned}
& M_{\text {pos }}(x)=\frac{x_{\max }-\mu}{\sigma}, \\
& M_{n e g}(x)=\frac{x_{\min }-\mu}{\sigma} .
\end{aligned}
$$

After that, the threshold is set to \pm 3 . We can consider the significance calculation in this way: the extent of the extreme value deviating from the average value $\mu$ of the characteristic parameters by the number of $\sigma$.

\section{Results and discussion}

The nickel-cobalt-manganese (NCM) ternary lithium-ion battery is used for this experiment. The battery module is a parallel connection of three batteries and then connected in series. The layout of the battery cells on the test rig is shown in Figure 3(a). The cell has a capacity of $50 \mathrm{Ah}$. The identification algorithm treats these three parallel groups as one series group with each cell having a capacity of $150 \mathrm{Ah}$. The equivalent resistance replacement method is employed to simulate the

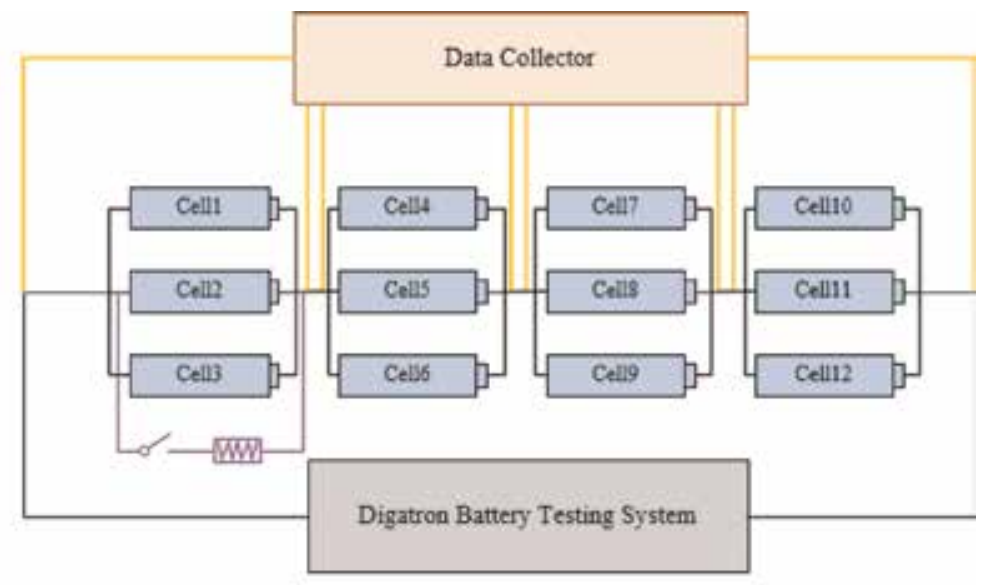

(a)

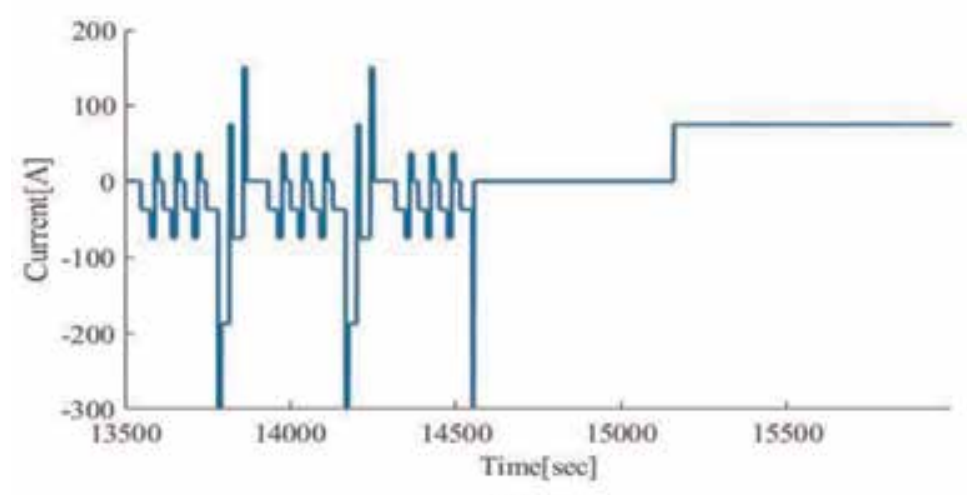

(b)

Figure 3 .

Configurations for the equivalent experiments: (a) layout of the battery module and (b) DST test cycle for the load configuration. 
electrical characteristics of the internal short circuit battery. The dynamic stress test (DST) cycle is used as an input. The DST working condition is shown in Figure 3(b), which can represent typical state changes of the battery under the practical operation conditions of electric vehicles. A data collector is used to measure the current and voltage of each cell. The accuracies for the current and voltage measurement are $0.1 \%$, and the sampling frequency is set to $1 \mathrm{~Hz}$. The maximum charge/discharge rate of the DST cycle is set to $2 \mathrm{C}$. When the minimum cell voltage decreases to $2.75 \mathrm{~V}$, the DST test is terminated. After 10 min rest, the battery pack is charged with a constant current of $75 \mathrm{~A}(0.5 \mathrm{C})$ until the voltage of any cell reaches $4.2 \mathrm{~V}$. After resting for another $10 \mathrm{~min}$, the next cycle is proceeded again.

An equivalent resistor with a precision of $0.1 \%$ is used in the experiment. The internal short circuit can be triggered or cancelled via a switch. In this experiment, three different values for the internal short circuit resistance are used including 1 , 10 , and $100 \Omega$, respectively. The results of the three resistances are used to validate the identification algorithm and compared with those of the previous method [12]. The terminal voltage and the current of each cell are measured and inputted to the internal short circuit identification algorithm programed in MATLAB.

\subsection{Experimental results}

The results of the modified identification method are given in Figures 4-6 for an external resistor of 1,10 , and $100 \Omega$, respectively. First, the measure values of
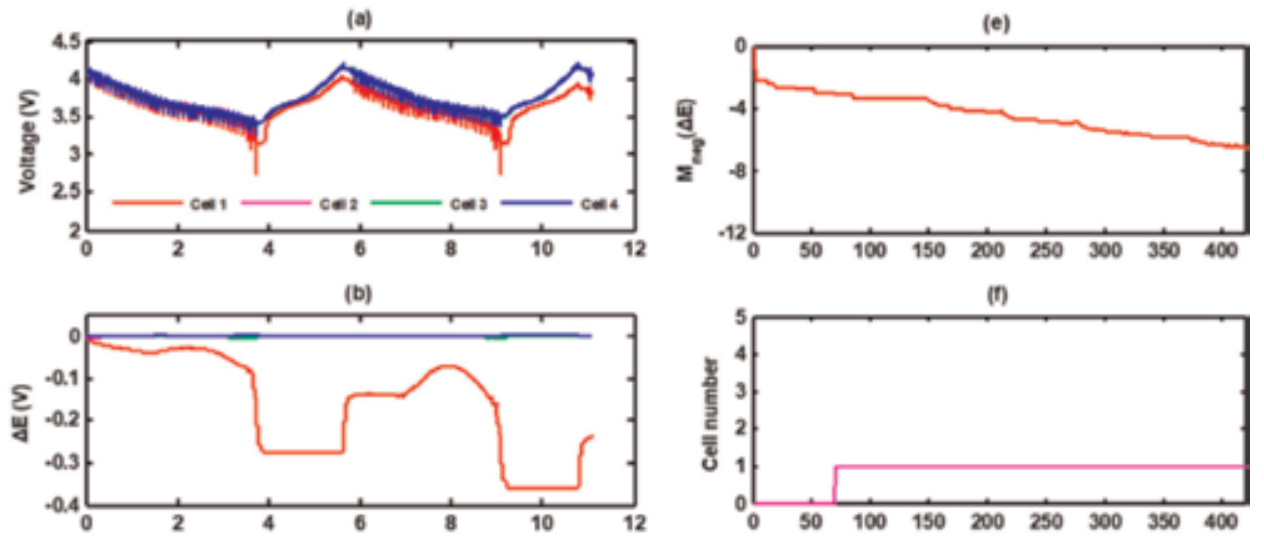

(c)
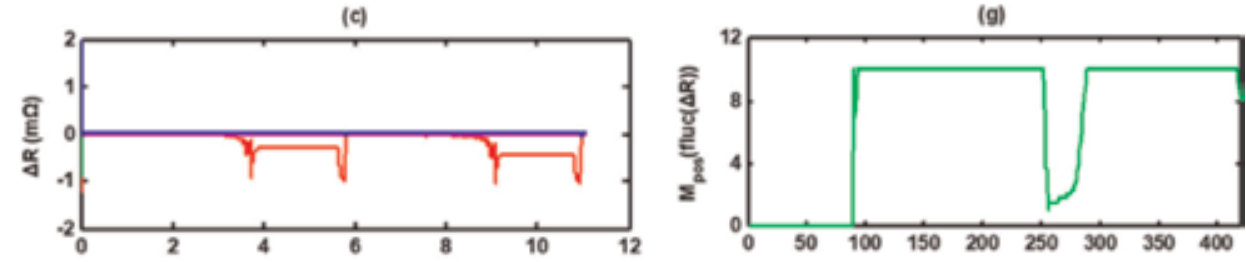

(d)
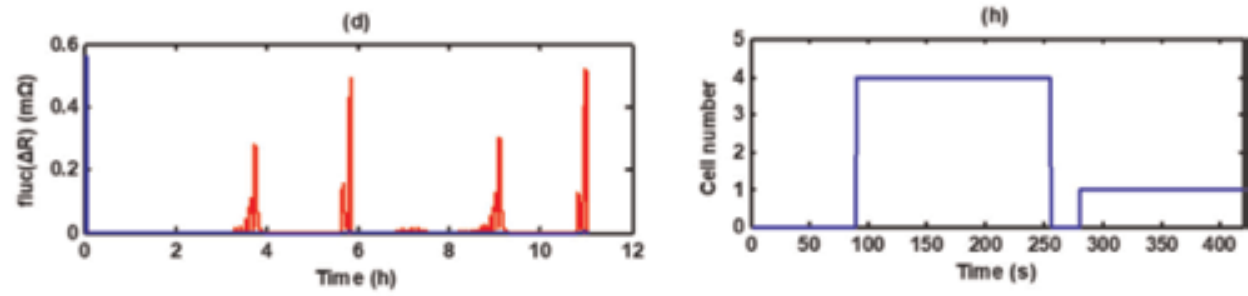

Figure 4.

Results of the equivalent experiment with an external resistor of $1 \Omega$ : (a) the measured terminal voltage,

(b) $\Delta E_{i},(c) \Delta R_{i},(d) f l u c\left(\Delta R_{i}\right)$, (e) the negative significance of $\Delta E$, $(f)$ the relative cell number of $M_{n e g}(\Delta E)$,

(g) the positive significance of fluc $(\Delta R)$, (h) the relative cell number of $M_{\text {pos }}(f l u c(\Delta R))$. 

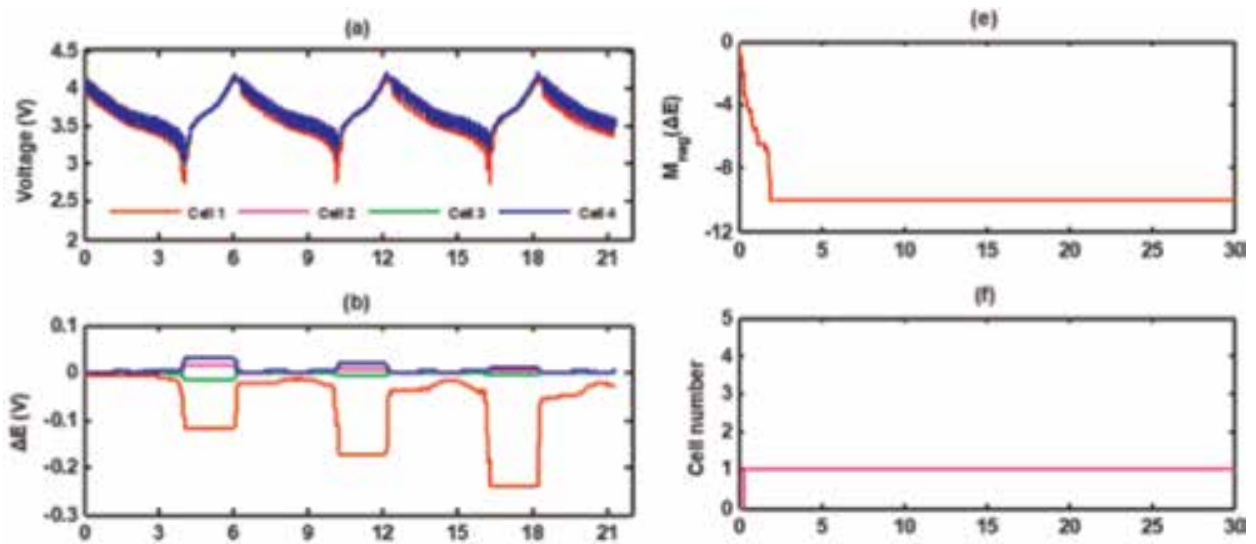

(c)

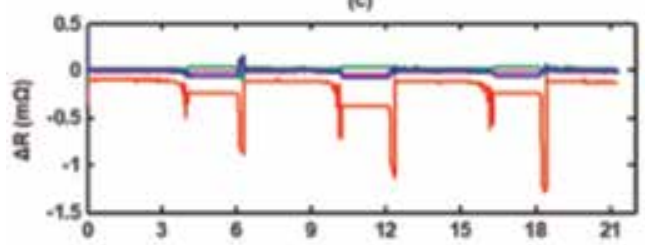

(a)

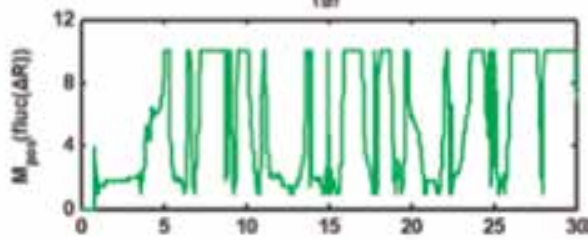

(d)
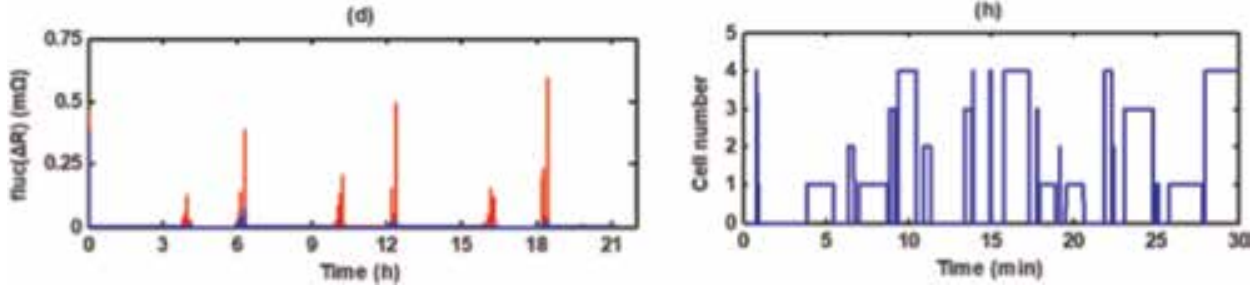

Figure 5 .

Results of the equivalent experiment with an external resistor of $10 \Omega$ : (a) the measured terminal voltage,

(b) $\Delta E_{i},(c) \Delta R_{i},(d)$ fluc $\left(\Delta R_{i}\right),(e)$ the negative significance of $\Delta E$, (f) the relative cell number of $M_{\text {neg }}(\Delta E)$,

$(g)$ the positive significance of fluc $(\Delta R)$, (h) the relative cell number of $M_{\text {pos }}(f l u c(\Delta R))$.

the terminal voltage are displayed. Then, $\Delta E_{i}$ and $\Delta R_{i}$ for each cell are determined by the mean-difference model and the RLS algorithm. Later, fluc $\left(\Delta R_{i}\right)$ is computed based on the values of $\Delta R_{i}$ and Eq. (13). It can be seen that the discharge power with an internal short circuit resistor of $1 \Omega$ is the largest, resulting in a greatest influence on the terminal voltage of the cell and the fluctuation of $\Delta R_{i}$.

For each case, the internal short circuit cell (i.e. Cell 1) already reaches the lowest voltage after the first DST cycle, leading to a much lower SOC. When the SOC drops to a very low value, the OCV is more sensitive. Therefore, a sudden drop of $\Delta E_{i}$ occurs at the end of the discharging process. The voltage of the short circuit cell recovers slightly after 10 min rest. Then, the battery pack undergoes a constant-current charging. The polarization internal resistance of the battery increases during this process, and the overvoltage rises for all the cells. The voltage for each cell decreases to a stable value gradually after another 10 min rest.

The change rate of $\Delta R_{i}$ increases at the end of the DST cycle owing to a low SOC state. At this moment, the cell has experienced a deep discharging process, and a large part of the lithium ions enter into the porous electrode. Effects of the electrochemical polarization and the concentration polarization weaken, leading to a sudden decrease of $\Delta R_{i}$. The results of $\Delta R_{i}$ keep constant during the constant-current charging process in this method. When the discharging begins again, it returns to its actual value. 
Investigation on Internal Short Circuit Identification of Lithium-Ion Battery Based on Mean... DOI: http://dx.doi.org/10.5772/intechopen.88534

The results for the detection time are listed in Table 1 and compared with those in [12]. It can be seen that the detection time of the modified identification method is much shorter than the previous one. The reason is mainly attributed to the judge criteria being relaxed. To prevent the occurrence of false alarm, both the conditions for $\Delta E_{i}$ and fluc $\left(\Delta R_{i}\right)$ are adopted simultaneously. The results indicate that the performance of the previous method for the internal short circuit detection can be improved significantly via this modification.
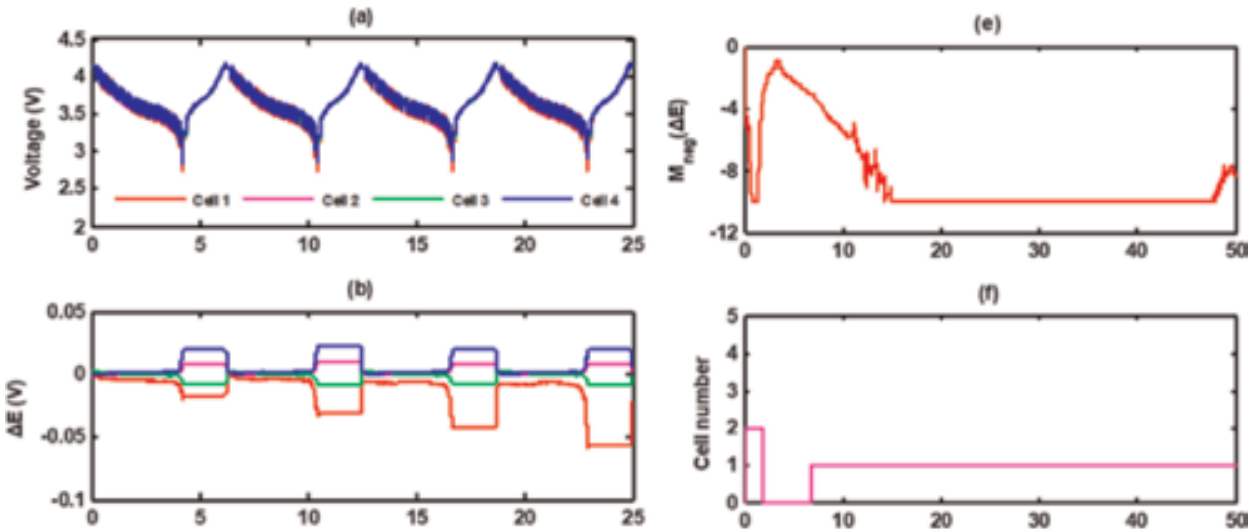

(c)
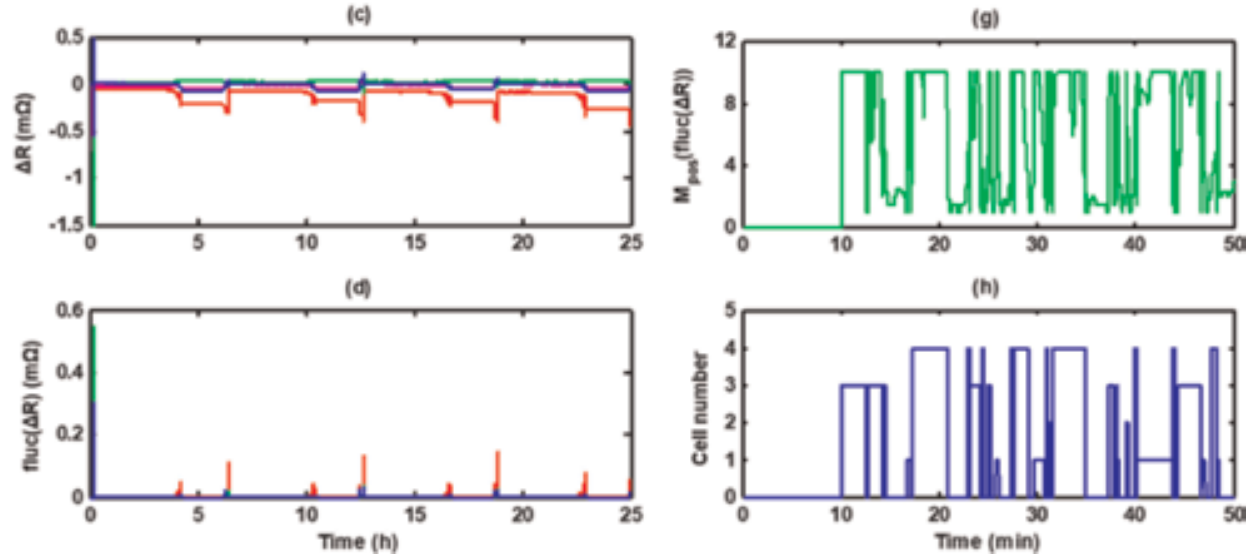

Figure 6.

Results of the equivalent experiment with an external resistor of $100 \Omega$ : (a) the measured terminal voltage,

(b) $\Delta E_{i},(c) \Delta R_{i},(d)$ fluc $\left(\Delta R_{i}\right)$, (e) the negative significance of $\Delta E$, (f) the relative cell number of $M_{n e g}(\Delta E)$,

$(g)$ the positive significance of fluc $(\Delta R)$, (h) the relative cell number of $M_{\text {pos }}($ fluc $(\Delta R))$.

\begin{tabular}{|c|c|c|c|c|}
\hline Parameter & Criteria & $100 \Omega$ & $10 \Omega$ & $1 \Omega$ \\
\hline$\Delta E_{i} \& f l u c\left(\Delta R_{i}\right)^{\mathrm{a}}$ & $\left(M_{\text {neg }}\left(\Delta E_{i}\right)<-3\right) \&\left(M_{\text {pos }}\left(\operatorname{fluc}\left(\Delta R_{i}\right)\right)>3\right)$ & $42 \mathrm{~min}$ & $27 \mathrm{~min}$ & $6 \min 40 s$ \\
\hline$\Delta E_{i}^{\mathrm{b}}$ & $M_{n e g}\left(\Delta E_{i}\right)<-6$ & $11 \mathrm{~h} 19 \mathrm{~min}$ & $1 \mathrm{~h} 18 \mathrm{~min}$ & $24 \mathrm{~min}$ \\
\hline $\operatorname{fluc}\left(\Delta R_{i}\right)^{\mathrm{b}}$ & $M_{p o s}\left(f l u c\left(\Delta R_{i}\right)\right)>10$ & $4 \mathrm{~h} 43 \mathrm{~min}$ & $1 \mathrm{~h} 17 \mathrm{~min}$ & $22 \min$ \\
\hline \multicolumn{2}{|l|}{ Improvement $(\%)^{\mathrm{c}}$} & $85.2 \%$ & $64.9 \%$ & $69.7 \%$ \\
\hline \multicolumn{5}{|c|}{$\begin{array}{l}{ }^{a} \text { Results of this study. } \\
{ }^{b} \text { Results of the previous method [12]. } \\
{ }^{c} \text { Improvement of a relative to } b .\end{array}$} \\
\hline
\end{tabular}

Table 1.

Experimental results and comparison with the previous method. 


\subsection{Analysis of significance test}

The results of the significance calculation for an external resistor of $1 \Omega$ are shown in the right column of Figure 4 . The significance value for $\Delta E_{i}$ is given in Figure 4(e), and the corresponding cell number is labelled in Figure 4(f). The significance of $\Delta E_{i}$ is negative due to the influences of the parameter and depleting effects. If the significance value is higher than -3 , no cell number is labelled, and a value of 0 is specified. The significance value for $f l u c\left(\Delta R_{i}\right)$ is positive and shown in Figure $4(\mathrm{~g})$. The significance value of $f l u c\left(\Delta R_{i}\right)$ is limited by a maximum value of 12 to give a clear exhibition. The results of the corresponding cell number are displayed in Figure 4(h). To enhance the reliability of this method, the anti-false alarm program ( $80 \%$ of the significant values among a continuous 150 points for both $\Delta E_{i}$ and fluc $\left(\Delta R_{i}\right)$ exceed the thresholds) is employed to confirm an event of the internal short circuit finally. In this experiment, the external resistor is connected by switching it on at the very beginning. The event of the internal short circuit is detected at the time of $400 \mathrm{~s}$. The results of the significance test for 10 and $100 \Omega$ are given in Figures 5 and 6, respectively. Likewise, a minimum of -12 is set if the negative significance is less than -12 . The significance value of $\Delta E_{i}$ can identify the right short circuit cell quickly. However, the results of $f l u c\left(\Delta R_{i}\right)$ vary dramatically and will take much longer to give the right outcome.
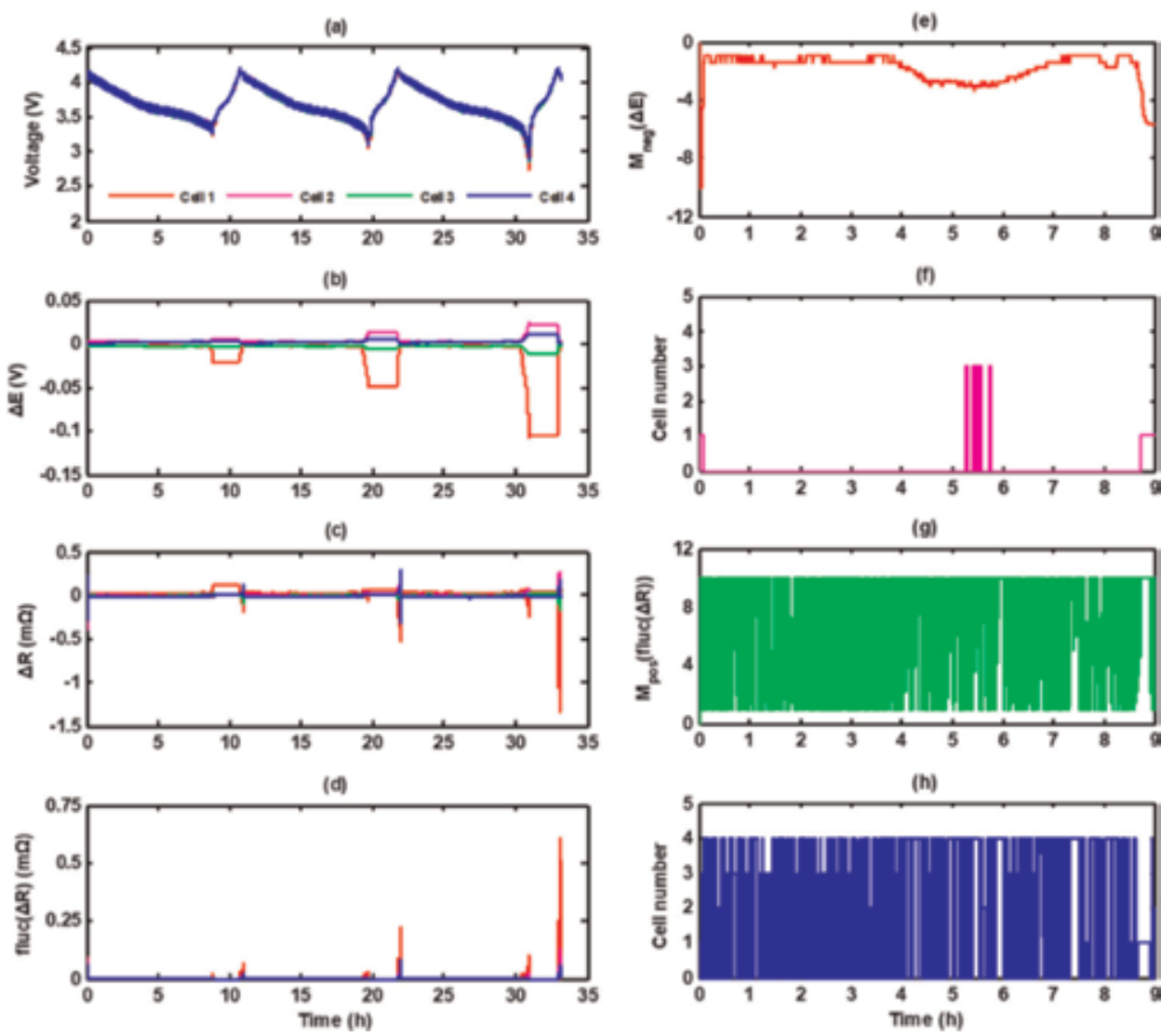

Figure 7.

Results of the equivalent experiment with an external resistor of $1000 \Omega$ : (a) the measured terminal voltage,

(b) $\Delta E_{i},(c) \Delta R_{i},(d)$ fluc $\left(\Delta R_{i}\right)$, (e) the negative significance of $\Delta E$, (f) the relative cell number of $M_{n e g}(\Delta E)$,

$(g)$ the positive significance of fluc $(\Delta R),(h)$ the relative cell number of $M_{\text {pos }}(f l u c(\Delta R))$ [21]. 


\subsection{Results for early stage identification}

If an internal short circuit can be detected at the beginning, protection measures can be adopted in time, and the system safety can be improved significantly. However, when a self-induced internal short circuit emerges at the early stage, the ISR resistance is very large. Accordingly, the variations of the OCV and the ISR current are very small. The possibility of this method for the early-stage internal short circuit identification is studied in this section [21]. An external resistance of $1000 \Omega$ with a $0.1 \%$ precision is used in this equivalent experiment. The results are shown in Figure 7. Because the variation magnitudes of $\Delta E_{i}$ and fluc $\left(\Delta R_{i}\right)$ are much smaller, the identification method cannot identify the internal short circuit by the significance test under the normal SOC range. If the SOC drops to a very low value, where the slope of OCV versus SOC is increased and the internal short circuit can be identified. After $8 \mathrm{~h}$ and $40 \mathrm{~min}$, the internal short circuit is detected for the case of $1000 \Omega$.

\subsection{Discussion}

The algorithm is based on the mean-difference model. There is a certain deviation in this model. In addition, the internal short circuit itself also generates noises for the identification algorithm. These may cause the internal resistance of the battery fluctuating more than other normal batteries. The true internal short circuit should be produced by the internal electrochemical reaction and the growth and change of the internal dendrites. At the same time, the temperature change caused by the internal short circuit will affect the electrochemical structure inside the battery, so the resistance fluctuation of the actual internal short circuit battery is larger than that of the normal battery. The external resistance used in the simulation of the internal short circuit may be different from the actual internal short circuit, but the equivalent experiment method still can be used to simulate the electrical characteristics of the internal short circuit battery. The battery resistance fluctuation of the equivalent experiment is smaller than the resistance fluctuation of the actual internal short circuit battery. As the internal short circuit battery ages, the lithium-ion and active materials in the battery decrease. The internal short circuit resistance of the battery fluctuates more, and the open circuit voltage will also drop faster.

The problem of internal short circuit detection caused by inconsistency is tricky, especially for the characteristic parameter of $\Delta E$. However, the evolution of the internal short circuit is a cumulative process. It is only possible to have an internal short circuit at a specific time and in a specific working condition. Generally, the internal short circuit occurs in a battery with poor consistency. Even if the internal short circuit occurs on a battery with a relatively high voltage, the internal short circuit will continuously self-discharge. The internal short circuit can be correctly recognized with the help of the characteristic parameter of $f l u c(\Delta R)$, although the time may become longer.

In the case of extreme SOC, the algorithm is limited by the inherent characteristics of the battery itself, and the internal short-circuited battery may be affected by the inconsistency, the polarization, and the solid-phase diffusion in the battery electrode particles at the extreme low SOC, causing the algorithm to produce a possible misjudgment. However, in electric vehicles, the working range of the battery generally would not reach the extreme SOC, so the algorithm can be used for internal short circuit identification in the normal driving range.

For the identification of early internal short circuit, it is difficult to separate the difference between internal short circuit and self-discharge. The voltage 
characteristics of self-discharge and internal short circuit are consistent. The threshold cannot be set to completely distinguish between self-discharge and internal short circuit. Therefore, early internal short circuit identification is easy to be misreported.

\title{
4. Conclusions
}

In this chapter, an identification method is presented for the internal short circuit of lithium-ion battery based on the mean-difference model and the recursive least square algorithm with a forgetting factor. This method is a modification of our previous investigation. The working principle and test procedure are described at first. Then, its performance is compared with that of the previous method. Subsequently, the influence of the parameters of the significance test on the detection time is analyzed. Finally, the possibility of this method for the early-stage internal short circuit identification is estimated.

The criteria of the significance test for the internal short circuit detection are adjusted, and two characteristic parameters $\left(\Delta E_{i}\right.$ and $\left.f l u c\left(\Delta R_{i}\right)\right)$ are employed to identify the internal short circuit simultaneously. Meanwhile, an anti-false alarm program is added to improve its reliability. Compared with the results of the previous study, the detection time can be shortened significantly by $64-85 \%$ via configuring the parameters of the significance test properly.

Because the self-induced internal short circuit resistance is very large at the early stage, this method cannot detect the internal short circuit event when the SOC is in the range of normal operation conditions. However, if the self-induced internal short circuit resistance reduces close to $100 \Omega$, the internal short circuit event can be identified successfully by this method, which can be integrated into the battery management system to monitor the operation of lithium-ion battery within the normal working range. A warning signal can be specified if an internal short circuit is detected so that the security of electric vehicles can be improved.

\section{Acknowledgements}

The authors would like to thank for the support of the National Natural Science Foundation of China (Grant No. U1564205 and 51876009), the Beijing Natural Science Foundation (Grant No. 3184052), and the Cooperation Project of Chongqing Changan New Energy Vehicle Technology Co., Ltd (No. 1811420009).

\section{Conflict of interest}

The authors declare no conflict of interest.

\author{
Abbreviations \\ DST dynamic stress test \\ ISC internal short circuit \\ NCM nickel-cobalt-manganese \\ OCV open circuit voltage \\ RLS recursive least square \\ SOC state of charge
}


Investigation on Internal Short Circuit Identification of Lithium-Ion Battery Based on Mean... DOI: $h t t p: / / d x$.doi.org/10.5772/intechopen.88534

\section{Author details}

Xu Zhang ${ }^{1}$, Yue Pan ${ }^{2}$, Enhua Wang ${ }^{1 *}$, Minggao Ouyang ${ }^{2}$, Languang Lu $^{2}$, Xuebing $\mathrm{Han}^{2}$, Guoqing $\mathrm{Jin}^{3}$, Anjian Zhou ${ }^{3}$ and Huiqian Yang ${ }^{3}$

1 School of Mechanical Engineering, Beijing Institute of Technology, Beijing, China

2 State Key Laboratory of Automotive Safety and Energy, Tsinghua University, Beijing, China

3 Chongqing Changan New Energy Vehicle Technology Co., Ltd, Chongqing, China

*Address all correspondence to: enhua.wang@yahoo.com

\section{IntechOpen}

(C) 2019 The Author(s). Licensee IntechOpen. This chapter is distributed under the terms of the Creative Commons Attribution License (http://creativecommons.org/licenses/ by/3.0), which permits unrestricted use, distribution, and reproduction in any medium, provided the original work is properly cited. (c) BY 


\section{References}

[1] Mehigan L, Deane JP, Gallachoir BP, Bertsch V. A review of the role of distributed generation (DG) in future electricity systems. Energy. 2018;163: 822-836

[2] Wang Q, Li S, Li R. China's dependency on foreign oil will exceed $80 \%$ by 2030: Developing a novel NMGM-ARIMA to forecast China's foreign oil dependence from two dimensions. Energy. 2018;163:151-167

[3] Li M, Xu H, Li W, Liu Y, Li F, Hu Y. The structure and control method of hybrid power source for electric vehicle. Energy. 2016;112:1273-1285

[4] Uddin K, Jackson T, Widanage WD, Chouchelamane G, Jennings PA, Marco J. On the possibility of extending the lifetime of lithium-ion batteries through optimal V2G facilitated by an integrated vehicle and smart-grid system. Energy. 2017;133:710-722

[5] Smiley A, Plett GL. An adaptive physics-based reduced-order model of an aged lithium-ion cell, selected using an interacting multiple model Kalman filter. Journal of Energy Storage. 2018; 19:120-134

[6] Kvasha A, Gutierrez C, Osa U, de Meatza I, Blazquez JA, Macicior H. A comparative study of thermal runaway of commercial lithium ion cells. Energy. 2018;159:547-557

[7] Chen K, Zheng F, Jiang J, Zhang W, Jiang Y, Chen K. Practical failure recognition model of lithium-ion batteries based on partial charging process. Energy. 2017;138:1199-1208

[8] Berrueta A, Urtasun A, Ursúa A, Sanchis P. A comprehensive model for lithiumion batteries: From the physical principles to an electrical model. Energy. 2018;144:286-300
[9] Fu Y, Lu S, Shi L, Cheng X, Zhang H. Ignition and combustion characteristics of lithium ion batteries under low atmospheric pressure. Energy. 2018;161: $38-45$

[10] Williard N, He W, Hendricks C, Pecht M. Lessons learned from the 787 dreamliner issue on lithium-ion battery reliability. Energies. 2013;6:4682-4695

[11] Ikeuchi A, Majima Y, Nakano I. Circuit and method for determining internal short-circuit, battery pack, and portable device. US20140184235A1; 2014

[12] Ouyang M, Zhang M, Feng X, Lu L, $\mathrm{Li} \mathrm{J,} \mathrm{He} \mathrm{X,} \mathrm{et} \mathrm{al.} \mathrm{Internal} \mathrm{short} \mathrm{circuit}$ detection for battery pack using equivalent parameter and consistency method. Journal of Power Sources. 2015; 294:272-283

[13] Feng X, Weng C, Ouyang M, Sun J. Online internal short circuit detection for a large format lithium-ion battery. Applied Energy. 2016;161:168-180

[14] Seo M, Goh T, Park M, Koo G, Kim SW. Detection of internal short circuit in lithium-ion battery using model-based switching model method. Energies. 2017;10:76

[15] Xia B, Shang Y, Nguyen T, Mi C. A correlation based detection method for internal short circuit in battery packs. In: 2017 IEEE Applied Power Electronics Conference and Exposition (APEC). 26-30 March 2017; Tampa, FL, USA

[16] Zhang Z, Kong X, Zheng Y, Zhou L, Lai $X$. Real-time diagnosis of microshort circuit for Li-ion batteries utilizing low-pass filters. Energy. 2019;166:

1013-1024

[17] Kang Y, Duan B, Zhou Z, Shang Y, Zhang C. A multi-fault diagnostic 
Investigation on Internal Short Circuit Identification of Lithium-Ion Battery Based on Mean...

DOI: http://dx.doi.org/10.5772/intechopen.88534

method based on an interleaved voltage measurement topology for series connected battery packs. Journal of Power Sources. 2019;417:132-144

[18] Kong X, Zheng Y, Ouyang M, Lu L, Li J, Zhang Z. Fault diagnosis and quantitative analysis of micro-short circuits for lithium-ion batteries in battery packs. Journal of Power Sources. 2018;395:358-368

[19] Feng X, He X, Lu L, Ouyang M. Analysis on the fault features for internal short circuit detection using an electrochemical-thermal coupled model. Journal of the Electrochemical Society. 2018;165:A155-A167

[20] Liu B, Jia Y, Li J, Yin S, Yuan C, $\mathrm{Hu} \mathrm{Z}$, et al. Safety issues caused by internal short circuits in lithium-ion batteries. Journal of Materials Chemistry A. 2018;6:21475-21484

[21] Zhang X, Pan Y, Wang E, Ouyang M. Investigation on early-stage internal short circuit identification for power battery pack. Advances in Engineering Research. 2019;184:233-235 



\title{
Induction Electrical Machine
} Simulation at Three-Phase Stator Reference Frame: Approach and Results

\author{
Mikhail Pustovetov
}

\begin{abstract}
This chapter provides the equations of mathematical model of three-phase induction electrical machine recorded in the three-phase stator reference frame. The possibilities of the proposed mathematical model are described in detail. This chapter also discusses the development of the computer model of an induction motor based on the abovementioned mathematical model. It uses an approach that allows combining during preparation the computer model dual methods: means of visual programming circuitry (in the form of electrical schematics) and logical one (in the form of block diagrams). The approach enables easy integration of the model of an induction motor as part of more complex models of electrical complexes and systems. The developed computer model gives the user access to the beginning and the end of a winding of each of the three phases of the stator and rotor. This property is particularly important when considering the asymmetric modes of operation or when powered by the special circuitry of semiconductor converters. Simulation results show the adequacy of the proposed mathematical model of induction electrical machine.
\end{abstract}

Keywords: induction electrical machine, mathematical model, stator reference frame, electromagnetic torque, instantaneous power, simulation

\section{Introduction}

The induction motor (IM) plays a very important role in industrial sectors and transport, primarily due to its robustness and low cost. There are some authors, who devote their publications to problems of mathematical modeling of IM [1-6]. The author wishes to offer its own version of a mathematical model (MM) of the IM, suitable for unbalanced modes of simulation purposes.

Writing the equations of the MM of a three-phase IM in a three-phase stator reference frame (SRF) is useful in the analysis, comparing the calculated and actual curves of currents and phase voltages, suitable without additional transformation of equations for the consideration of modes of IM operation at asymmetrical characteristics of feed or parameters of IM. Simulation results for the coordinates $\alpha, \beta, \gamma$ correspond to the actual processes in phases A, B, and C of the stator, in the case of the short-circuited rotor, i.e., in most cases, only and can be experimentally 
measured. IM simulation in three-phase coordinates is useful in detecting and diagnosing defects in the stator [3] and implementing algorithms for direct torque control.

It can be argued-subject to review only, the fundamental spatial harmonics of the magnetic field in the air gap is symmetrical in a construction three-phase IM, powered by non-sinusoidal voltages asymmetrical system - that simulation results will be correct, but the same one in two-phase orthogonal coordinates will not.

\section{Mathematical model equations}

The suggested MM of a three-phase IM is based on a three-phase electrical machine MM at SRF coordinate system axis $\alpha, \beta, \gamma[5]$, which are aligned with the stator phase axes A, B, and C. This basic MM supplemented by iron losses resistances $r_{\mu}$ included in magnetization circuits in each phase of IM in parallel with magnetizing inductance $L_{\mu}[6]$ (analogy with the T-shaped equivalent circuit of induction electric machine). The equations of the electromagnetic processes in IM are given in Eq. (1), adopted by the usual generalized electrical machine assumptions: each of the phase stator windings creates in a smooth air gap in the sinusoidal-allocated magnetomotive force; magnetic saturation coefficient is constant. In the expressions (Eq. (1)), the following notations are further adopted: $v$, voltage; $i$, current; $t$, time; $r$, resistance; $\Psi$, magnetic flux linkage; $\omega_{r}$, mechanical rotor speed; and $p$, the number of pole pairs. Lower indexes $\alpha, \beta, \gamma$ indicate the affiliation to the appropriate phase. The subscript $s$ indicates the affiliation to the stator, the index $r$ belongs to the rotor, and index $\mu$ belongs to the magnetization branch. $L_{\sigma s \alpha}$ is the leakage inductance of stator phase; $L_{\sigma r \alpha}$ is the leakage inductance of the rotor winding phase.

Traditionally, the rotor parameters are given to the stator winding. Detailed components of the system (Eq. (1)) are described in Eqs. (2)-(7). The form of Eq. (1) as much as possible is unified with published equations (Eq. (1)) of a threephase transformer MM [7]:

$$
\begin{cases}v_{s \alpha}=r_{s \alpha} i_{s \alpha}+\frac{d\left(L_{\sigma s \alpha} i_{s \alpha}\right)}{d t}+v_{0 \alpha} ; & i_{\mu \alpha \_a c t i v e}=\frac{v_{0 \alpha}}{r_{\mu \alpha}} ; \\ v_{s \beta}=r_{s \beta} i_{s \beta}+\frac{d\left(L_{\sigma s \beta} i_{s \beta}\right)}{d t}+v_{0 \beta} ; & i_{\mu \beta \_a c t i v e}=\frac{v_{0 \beta}}{r_{\mu \beta}} ; \\ v_{s \gamma}=r_{s \gamma} i_{s \gamma}+\frac{d\left(L_{\sigma s \gamma} i_{s \gamma}\right)}{d t}+v_{0 \gamma} ; \quad i_{\mu \gamma \_a c t i v e}=\frac{v_{0 \gamma}}{r_{\mu \gamma}} ; \\ v_{r \alpha}=e_{0 \alpha}-e_{r o t \alpha}-\frac{d\left(L_{\sigma r \alpha} i_{r \alpha}\right)}{d t}-r_{r \alpha} i_{r \alpha} ; \\ v_{r \beta}=e_{0 \beta}-e_{r o t \beta}-\frac{d\left(L_{\sigma \gamma \beta} i_{r \beta}\right)}{d t}-r_{r \beta} i_{r \beta} ; \\ v_{r \gamma}=e_{0 \gamma}-e_{r o t \gamma}-\frac{d\left(L_{\sigma \gamma \gamma} i_{r \gamma}\right)}{d t}-r_{r \gamma} i_{r \gamma \gamma} ;\end{cases}
$$

Voltages at the terminals of phase magnetization branches (derived from flux linkage of mutual induction)

$$
v_{0 \alpha}=\frac{d \Psi_{\mu \alpha}}{d t}=r_{\mu \alpha}\left[\left(i_{s \alpha}+i_{r \alpha}\right)-\frac{1}{2}\left(i_{s \beta}+i_{r \beta}+i_{s \gamma}+i_{r \gamma}\right)-\frac{\Psi_{\mu \alpha}}{M}\right] ;
$$


Induction Electrical Machine Simulation at Three-Phase Stator Reference Frame: Approach... DOI: http://dx.doi.org/10.5772/intechopen.88906

$$
\begin{aligned}
& v_{0 \beta}=\frac{d \Psi_{\mu \beta}}{d t}=r_{\mu \beta}\left[\left(i_{s \beta}+i_{r \beta}\right)-\frac{1}{2}\left(i_{s \alpha}+i_{r \alpha}+i_{s \gamma}+i_{r \gamma}\right)-\frac{\Psi_{\mu \beta}}{M}\right] ; \\
& v_{0 \gamma}=\frac{d \Psi_{\mu \gamma}}{d t}=r_{\mu \gamma}\left[\left(i_{s \gamma}+i_{r \gamma}\right)-\frac{1}{2}\left(i_{s \alpha}+i_{r \alpha}+i_{s \beta}+i_{r \beta}\right)-\frac{\Psi_{\mu \gamma}}{M}\right] .
\end{aligned}
$$

Mutual inductance of IM windings defined as

$$
M=(2 / 3) L_{\mu}
$$

$M$-mutual inductance of the phase windings of the rotor and stator of IM in the case of coincidence of their axes. In other words main inductance of the winding of the stator when magnetic flux calculated in the absence of currents in other phases of the stator and rotor windings (i.e., from the part of main magnetic flux created by the stator phase itself).

$L_{\mu}$-complete phase inductance of the stator winding from the main magnetic flux, which takes into account the presence of currents in other phases. In other words the inductance of the main magnetic flux part is created by the winding itself $M$, and the inductance of the portion of the main magnetic flux is created by two other stator windings $M / 2$.

Per-phase magnetizing currents:

$$
\begin{aligned}
i_{\mu \alpha} & =i_{s \alpha}+i_{r \alpha}=\frac{2}{3}\left[\left(i_{s \alpha}-\frac{1}{2}\left(i_{s \beta}+i_{s \gamma}\right)\right)+\left(i_{r \alpha}-\frac{1}{2}\left(i_{r \beta}+i_{r \gamma}\right)\right)\right] \\
& =i_{\mu \alpha \_a c t i v e}+i_{\mu \alpha \_ \text {reactive }} ; \\
i_{\mu \beta} & =i_{s \beta}+i_{r \beta}=\frac{2}{3}\left[\left(i_{s \beta}-\frac{1}{2}\left(i_{s \alpha}+i_{s \gamma}\right)\right)+\left(i_{r \beta}-\frac{1}{2}\left(i_{r \alpha}+i_{r \gamma}\right)\right)\right] \\
& =i_{\mu \beta \_a c t i v e}+i_{\mu \beta \_r e a c t i v e} ; \\
i_{\mu \gamma} & =i_{s \gamma}+i_{r \gamma}=\frac{2}{3}\left[\left(i_{s \gamma}-\frac{1}{2}\left(i_{s \alpha}+i_{s \beta}\right)\right)+\left(i_{r \gamma}-\frac{1}{2}\left(i_{r \alpha}+i_{r \beta}\right)\right)\right] \\
& =i_{\mu \gamma \_a c t i v e}+i_{\mu \gamma \_ \text {reactive }} .
\end{aligned}
$$

In the expressions (Eq. (4)), $i_{\mu_{\_} \text {active }}$ and $i_{\mu_{-} \text {reactive }}$ are active and reactive (inductive) components of per-phase magnetizing current, respectively.

$$
\begin{gathered}
e_{0 \alpha}=-v_{0 \alpha} ; \\
e_{0 \beta}=-v_{0 \beta} ; \\
e_{0 \gamma}=-v_{0 \gamma} . \\
e_{r o t \alpha}=\frac{\left(\Psi_{r \beta}-\Psi_{r \gamma}\right) p \omega_{r}}{\sqrt{3}} ; \\
e_{r o t \beta}=\frac{\left(\Psi_{r \gamma}-\Psi_{r \alpha}\right) p \omega_{r}}{\sqrt{3}} ; \\
e_{r o t \gamma}=\frac{\left(\Psi_{r \alpha}-\Psi_{r \beta}\right) p \omega_{r}}{\sqrt{3}} .
\end{gathered}
$$


Flux linkages of IM rotor phases:

$$
\begin{aligned}
& \Psi_{r \alpha}=L_{\sigma r \alpha} i_{r \alpha}+\Psi_{\mu \alpha} ; \\
& \Psi_{r \beta}=L_{\sigma r \beta} i_{r \beta}+\Psi_{\mu \beta} ; \\
& \Psi_{r \gamma}=L_{\sigma r \gamma} i_{r \gamma}+\Psi_{\mu \gamma} .
\end{aligned}
$$

The electromagnetic torque of IM equation is

$$
\begin{aligned}
T_{\mathrm{em}}= & p \frac{\sqrt{3}}{2} M\left[\left(i_{s \alpha} i_{r \gamma}+i_{s \beta} i_{r \alpha}+i_{s \gamma} i_{r \beta}\right)\right. \\
& \left.-\left(i_{s \alpha} i_{r \beta}+i_{s \beta} i_{r \gamma}+i_{s \gamma} i_{r \alpha}\right)\right] .
\end{aligned}
$$

The equation of motion for the IM shaft in the case of single-mass mechanical part:

$$
\frac{d \omega_{r}}{d t}=\frac{1}{J}\left(T_{e m}-T_{l o a d}\right)
$$

where $J$ is the moment of inertia of masses coupling with the rotor shaft and $T_{\text {load }}$ is the static torque of mechanical load coupling with the IM shaft.

In the general case, Eq. (9) can be written as

$$
\frac{d \omega_{r}}{d t}=\frac{1}{J}\left(T_{\text {em }}-T_{\text {load }}\left(t, \omega_{r}, \Theta_{r}\right)\right)=\frac{T_{\text {dynamic }}}{J},
$$

$T_{\text {dynamic }}-$ dynamic torque on the IM shaft;

$$
\omega_{r}=\omega_{r 0}+\int_{0}^{t} \frac{T_{\text {dynamic }}}{J} d t,
$$

$\omega_{r 0}$-initial angular speed of the rotor shaft of IM.

The angle of rotation of the IM rotor shaft, radian

$$
\Theta_{r}=\Theta_{r 0}+\int_{0}^{t} \omega_{r} d t
$$

$\Theta_{r 0}$-initial angular position of the IM rotor shaft, radian.

It is interesting to know the energy performance of the electric machine in the transition process. For IM in the absence of power from the rotor instantaneous value of the active power consumption is calculated as

$$
P_{1}=v_{s \alpha} i_{s \alpha}+v_{s \beta} i_{s \beta}+v_{s \gamma} i_{s \gamma}
$$

The instantaneous reactive power consumption

$$
Q_{1}=-\frac{1}{\sqrt{3}}\left[v_{s \alpha}\left(i_{s \beta}-i_{s \gamma}\right)+v_{s \beta}\left(i_{s \gamma}-i_{s \alpha}\right)+v_{s \gamma}\left(i_{s \alpha}-i_{s \beta}\right)\right]
$$

The instantaneous value of useful shaft power of IM

$$
P_{2}=\omega_{r} T_{\text {load }}
$$

The abovementioned equations (Eqs. (1)-(15)) of IM MM may be supplemented by the expressions (Eq. (16)), which allow to go to the description of the rotor 


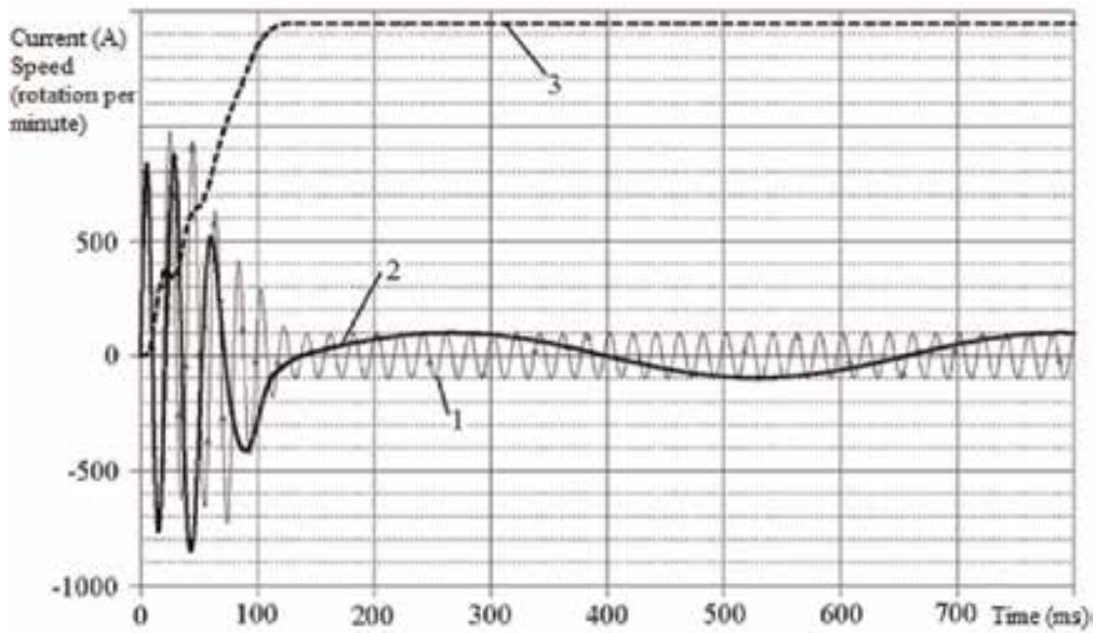

Figure 1.

The results of the rotor phase current simulation for IM type AE92-4.

phase currents at frequency of $f_{2}=s \cdot f_{1}$, where $s$ is the sleep of the rotor and $f_{1}$ is the frequency of stator voltage.

$$
\begin{aligned}
i_{r a}= & i_{r \alpha}\left(\frac{1}{3}+\frac{2}{3} \cos \left(p \cdot \Theta_{r}\right)\right)+i_{r \beta}\left(\frac{1}{3}+\frac{2}{3} \cos \left(p \cdot \Theta_{r}-\frac{2 \pi}{3}\right)\right) \\
& +i_{r \gamma}\left(\frac{1}{3}+\frac{2}{3} \cos \left(p \cdot \Theta_{r}+\frac{2 \pi}{3}\right)\right) ; \\
i_{r b}= & i_{r \alpha}\left(\frac{1}{3}+\frac{2}{3} \cos \left(p \cdot \Theta_{r}+\frac{2 \pi}{3}\right)\right)+i_{r \beta}\left(\frac{1}{3}+\frac{2}{3} \cos \left(p \cdot \Theta_{r}\right)\right) \\
& +i_{r \gamma}\left(\frac{1}{3}+\frac{2}{3} \cos \left(p \cdot \Theta_{r}-\frac{2 \pi}{3}\right)\right) ; \\
i_{r c}= & i_{r \alpha}\left(\frac{1}{3}+\frac{2}{3} \cos \left(p \cdot \Theta_{r}-\frac{2 \pi}{3}\right)\right) \\
& +i_{r \beta}\left(\frac{1}{3}+\frac{2}{3} \cos \left(p \cdot \Theta_{r}+\frac{2 \pi}{3}\right)\right)+i_{r \gamma}\left(\frac{1}{3}+\frac{2}{3} \cos \left(p \cdot \Theta_{r}\right)\right) .
\end{aligned}
$$

Such a representation of the rotor currents demonstrates the nature of changes in the transition processes (see Figure 1, which shows the results of the rotor phase current simulation in the case of balanced power supply sinusoidal voltages for IM type AE92-4, 4-pole, $40 \mathrm{~kW}$ ). In Figure 1, curve 1 is $i_{r \gamma}$ current, curve 2 is $i_{r c}$ current, and curve 3 is rotor speed. According to the frequency $f_{2}$, you can check the value of the rotor speed of IM.

Figure 2 shows the applicability of the developed MM for the simulation of electromagnetic and electromechanical processes in IM with its power supply from the power semiconductor converter-autonomous voltage inverter.

\section{Requirements for computer model}

In the process of the computer model of the IM according to the equations (Eqs. (1)-(12)), development using OrCAD—computer-aided design system- 


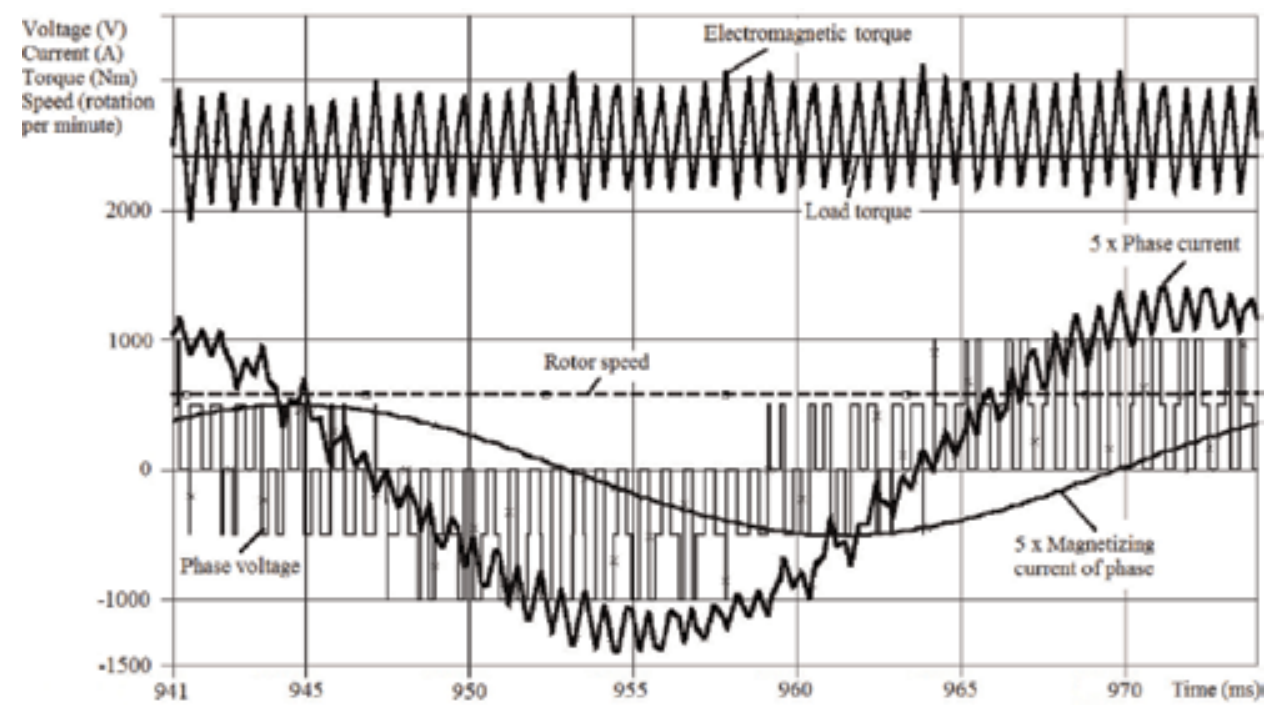

Figure 2.

The results of simulation for traction IM type DTA-350M (6-pole, $350 \mathrm{~kW})$ under power supply from autonomous voltage inverter condition.

intended primarily for the design and simulation of electronic and electrical devices [8], there was the task of ensuring the embedded model of IM in an electrical power supply circuit, including converters, by simple connection of virtual terminals. That is, relative to the model of the electrical circuit that is attached to this circuit, the IM model should also have the properties of electric circuits: one can apply a potential difference to the terminals, providing a bidirectional electric current in the connecting circuits, including a pass-through current between them. Another task is the formation of such structure of the computer model which will be used perhaps as a universal template, which records the values of variables (parameters of IM), asked simultaneously for all equations. The resulting computer model is suitable for the description of both the squirrel-cage and wound rotor IM in any of four quadrants. The winding phases can be connected in a triangle scheme, wye, independently joined with each of its voltage to have any other wiring of each other or of power source. The developed computer model gives the user access to the beginning and the end of a winding of each of the three phases of the stator and the rotor. This property is particularly important when considering the asymmetric modes of operation or when powered by the special circuitry of semiconductor converters [9].

\section{The implementation of a computer model}

The tasks are solved by combining two approaches in the IM computer model development: circuit for electrical parts and the method of block diagrams for the magnetic and mechanical parts. The computer model of IM is packed into a hierarchical block with a specified list of variables (parameters of IM). Within a single OrCAD project, using the copy operation, one can get the required number of hierarchical blocks (models of IM), for each of which it is possible to set unique parameter values. Sensors and signal inputs in the IM model used controlled sources of currents and voltages. Such voltage source is controlled by current (VSCC) and by voltage (VSCV), and current source is controlled by voltage (CSCV). A graphical image of the computer model of phase $A$ windings of the stator and the rotor of IM, composed of Eqs. (1)-(7), is shown in Figure 3. 

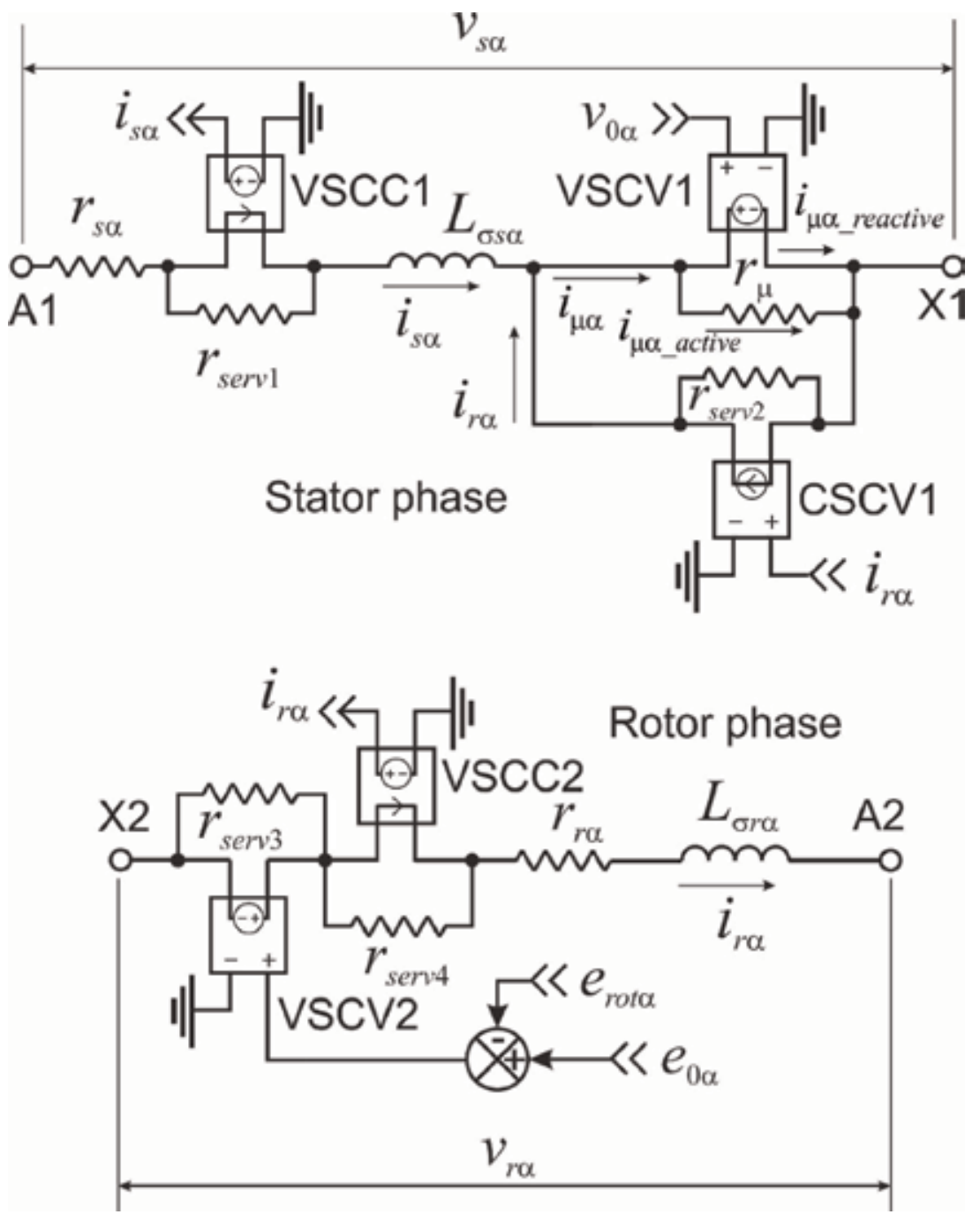

Figure 3 .

The graphical image of the computer model of phase A windings of the stator and the rotor of IM.

VSCC1 performs a function of the phase current of the stator $i_{s \alpha}$ sensor; similarly, VSCC2 is a sensor of rotor phase current $i_{r \alpha}$ given to the stator winding. CSCV1 is used for entering $i_{r \alpha}$ into the scheme. VSCV1 is introduced into the phase magnetizing circuit $v_{0 \alpha}$ voltage. A similar function is performed by VSCV2 entering into the circuit of the rotor winding; the difference is between the electromotive force of a branch of magnetization and rotation electromotive force, i.e., the voltage drop value. Active resistances $r_{\text {serv }}$ (see Figure 3) have a large value, for example, $10 \mathrm{M} \Omega$. These resistors are introduced for service purposes. Without affecting numerical calculation results, they stabilize the solution (simulation) by maintaining the current circuit (the physical sense-the way for leakage current), which is especially important when a discrete change of the resistance of a IM circuit occurs, for example, when powered from the frequency converter or in the case of the phase circuit breaking. A similar solution is described in [10].

The rest of the equations of a three-phase IM mathematical model is implemented by the author in the computer model in the form of block diagrams [11]. Figure 4 shows a part of the model, where one obtains instantaneous value of $L_{\mu} *$-complete phase inductance of the stator winding from the main magnetic 


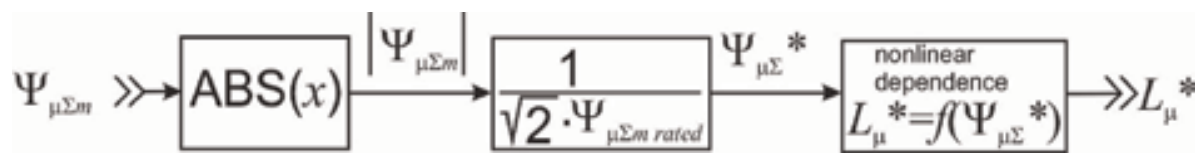

Figure 4.

The block diagram for obtaining instantaneous values of complete phase inductance of the stator winding from the main magnetic flux in relative units $L_{\mu} *$.

flux in relative units. $\Psi_{\mu \Sigma m r a t e d}$ is the rated value of $\Psi_{\mu \Sigma m}$ - the instantaneous value of the amplitude of the representing vector of the flux linkage of mutual induction.

Signal $\Psi_{\mu \Sigma m}$ can be calculated as

$$
\Psi_{\mu \Sigma m}=\sqrt{\Psi_{\mu x}^{2}+\Psi_{\mu y}^{2}},
$$

where $\Psi_{\mu x}$ and $\Psi_{\mu y}$ are the projections of the representing vector of the flux linkage of mutual induction on orthogonal coordinate axes X and $\mathrm{Y}$. In the case of three-phase stator reference frame of the coordinate system axis, these projections are derived from mutual induction flux linkages of each phase.

$$
\begin{gathered}
\Psi_{\mu x}=\frac{2}{3}\left(\Psi_{\mu \alpha}+\Psi_{\mu \beta} \cos \left(-\frac{2 \pi}{3}\right)+\Psi_{\mu \gamma} \cos \left(\frac{2 \pi}{3}\right)\right) ; \\
\Psi_{\mu y}=-\frac{2}{3}\left(\Psi_{\mu \beta} \sin \left(-\frac{2 \pi}{3}\right)+\Psi_{\mu \gamma} \sin \left(\frac{2 \pi}{3}\right)\right) .
\end{gathered}
$$

Figure 5 shows the piecewise linear approximation by five points of $L_{\mu} *\left(\Psi_{\mu \Sigma} *\right)$ for IM type AGV250 (2-pole, $110 \mathrm{~kW}$ ). Such a way successfully tested in IM simulator is developed by means of OrCAD PSpice [8]: the one standard component table is used for programming the abovementioned approximated dependence.

In Figure 6 there is the model part corresponding to the abovementioned (Eqs. (1)-(3)) equations. Figure 7 shows a part of the IM model, designed to determine the rotation electromotive force in each phase. Model rotor speed $\omega_{r_{-} \text {model }}$ in $p$ times is higher than the real mechanical speed of rotor $\omega_{r}$. Figure 8 shows a

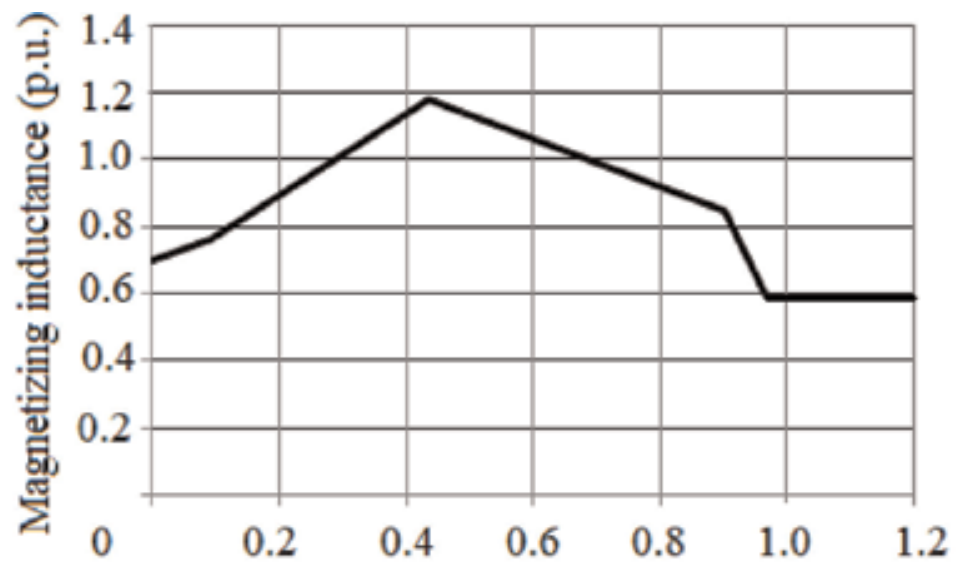

Instantaneous value of the magnitude of the representing vector of the flux linkage of mutual induction (p.u.)

Figure 5.

The piecewise linear approximation by five points of $L_{\mu} *\left(\Psi_{\mu \Sigma m} *\right)$ for IM type AGV250. 
Induction Electrical Machine Simulation at Three-Phase Stator Reference Frame: Approach... DOI: http://dx.doi.org/10.5772/intechopen.88906

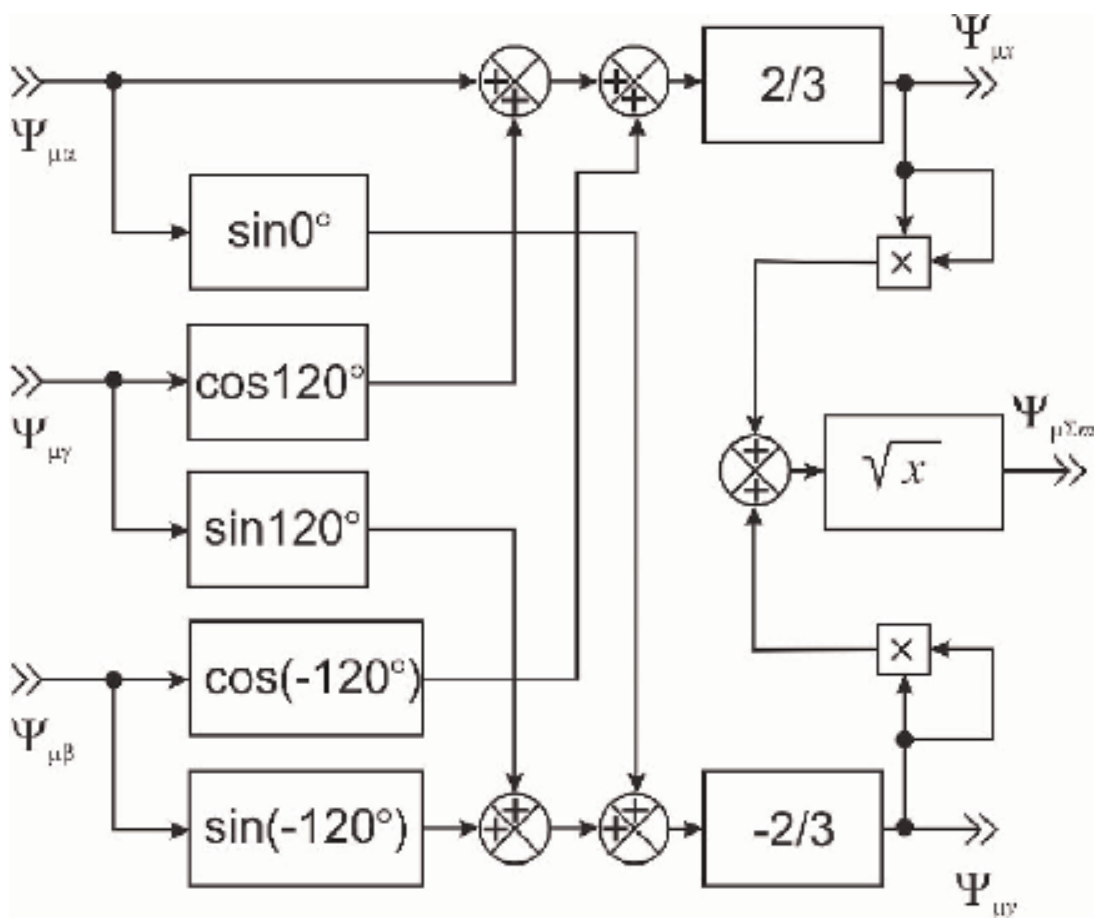

Figure 6.

The block diagram that implements the calculation of the instantaneous value of the amplitude of the representing vector of the flux linkage of mutual induction.

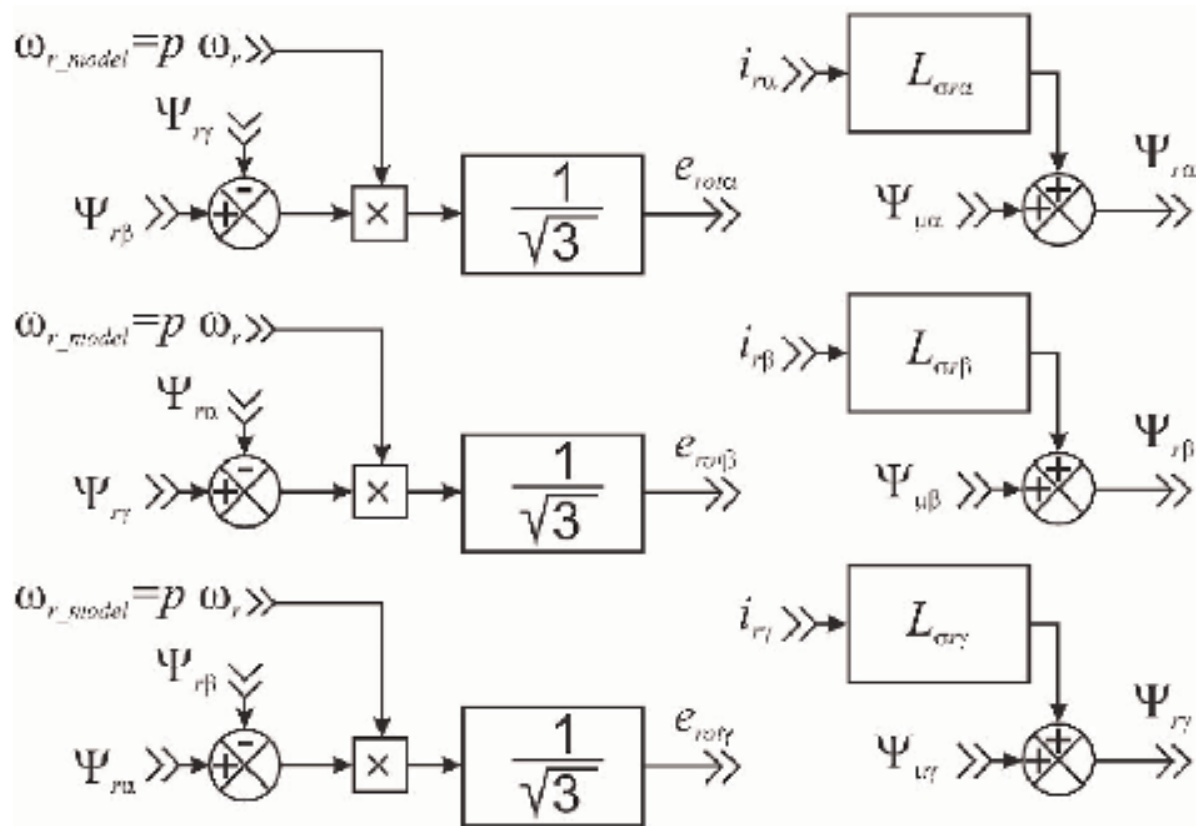

Figure 7.

Part of the IM model, designed to determine the rotation electromotive force in each phase.

part of the IM model corresponding to equations (Eq. (2)). Figure 9 shows a part of the model, which forms the signals of electromagnetic torque $T_{e m}$, angular speed $\omega_{r}$, and the angle of rotation $\Theta_{r}$ of the rotor. In Figure $9, n_{r}$ is mechanical angular rotor speed, rotation per minute. 


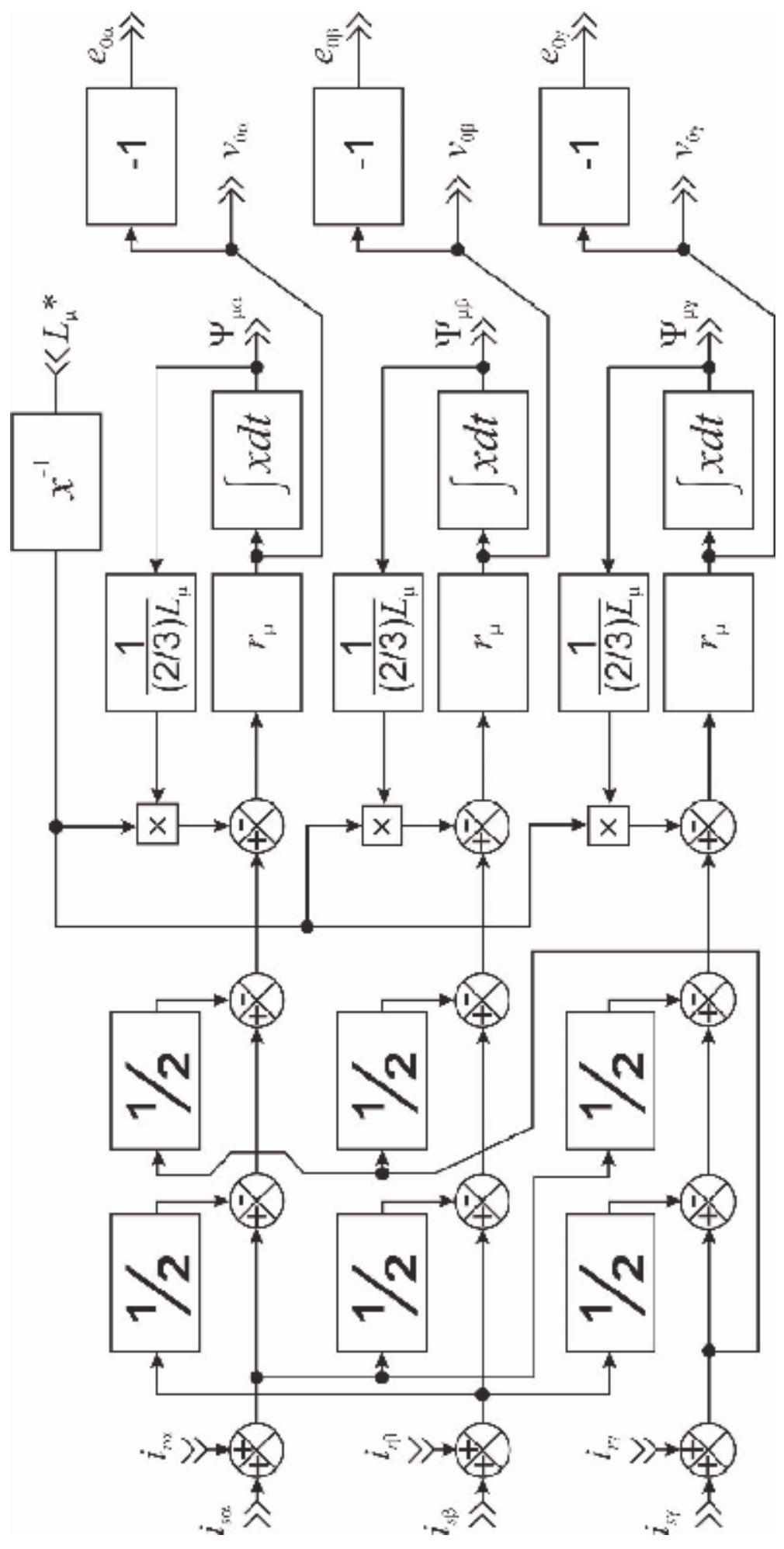

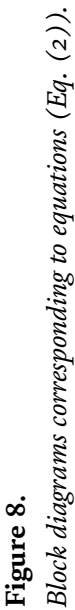




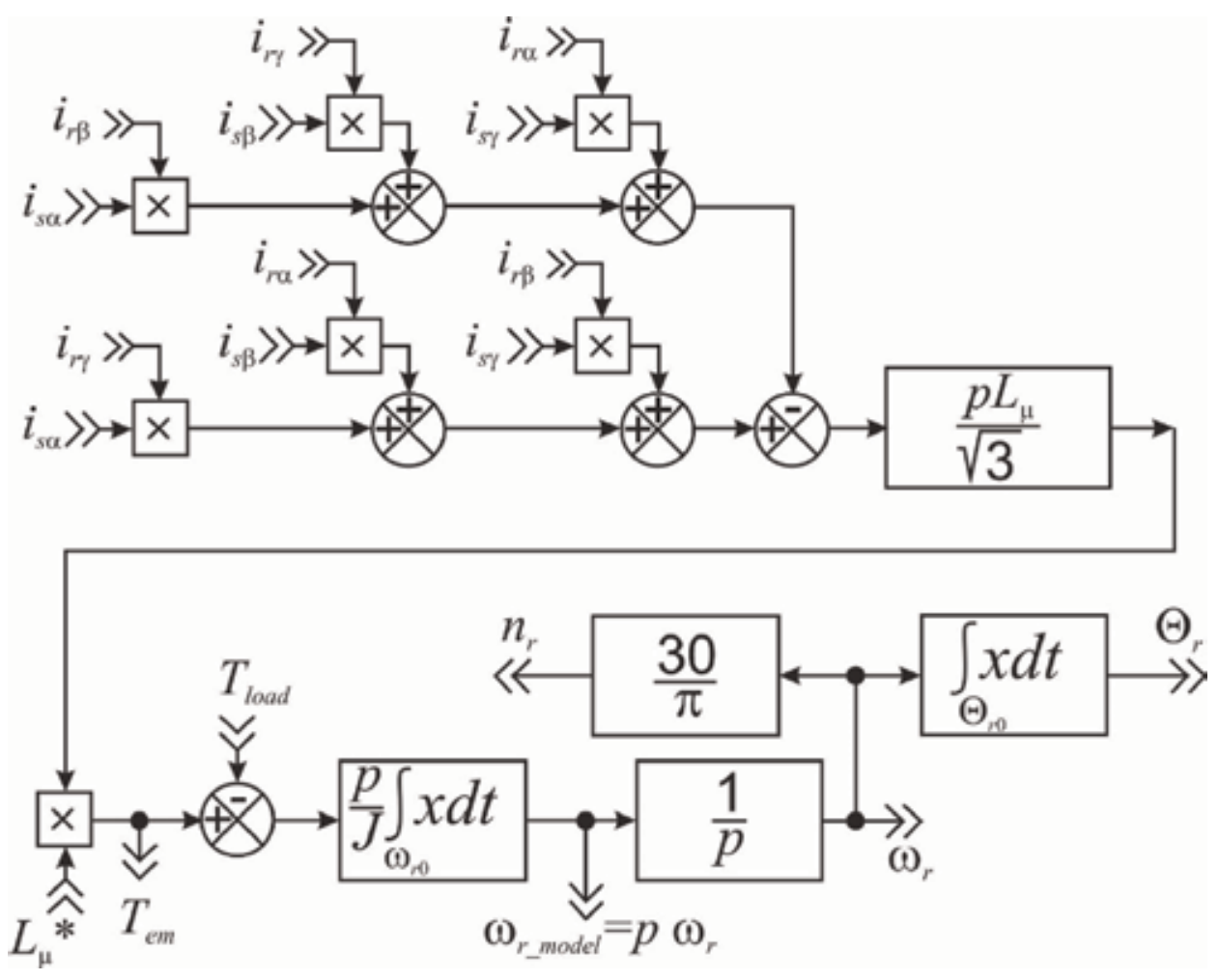

Figure 9.

Block diagrams, which form the signals of electromagnetic torque $T_{e m}$, angular speed $\omega_{r}$, and the angle of rotation of rotor $\Theta_{r}$.

\section{An example of using a mathematical model of the motor when the power supply voltage is unbalanced}

A good example of functionality for previously provided MM under unbalanced supply voltage maybe modeling of electromechanical phase splitter, made on the basis of a three-phase IM.

Electromechanical phase splitters are used in Russia and India [12] onboard of electric trains and electric locomotives for transform AC single-phase voltage in three-phase voltage to feed auxiliary electric drives with IM loaded by fans and air compressors. Figure 10 is a schematic diagram of the rotary phase splitter, where $C_{1}$ is operating and $C_{2}$ is start-up capacity.

Phase splitters are IM with a symmetrical or nonsymmetrical stator winding and no load (or low load) on the shaft. Phase splitter can be considered as combined single-phase IM and three-phase synchronous generator. In accordance with the terminology, adopted in India, the phase splitter is called the ARNO converter [12].

On contemporary freight AC electric locomotives of family "Ermak" in Russia, IM of NVA-55 type (4-pole, $55 \mathrm{~kW}$ ) is used as a phase splitter. To drive the fans and compressor, the same type of IM is used. Let us make simulation of the phase splitter start without connecting electrical loads to check for phase splitting effect. Define $C_{1}=968 \mathrm{uF}$ and $C_{2}=2904 \mathrm{uF}$. The torque of mechanical losses on the shaft (of the load) will take $28 \mathrm{Nm}$ at the rotor speed of 1500 rotation per minute.

The simulated results of phase splitter start are shown in Figures $\mathbf{1 1}$ and 12, where the graphs are 1, 2, 3-line voltages between phases $A-B, B-C, C-A-$ and 4 , rotor speed. Start-up capacity $C_{2}$ is switched off when the current value of the 


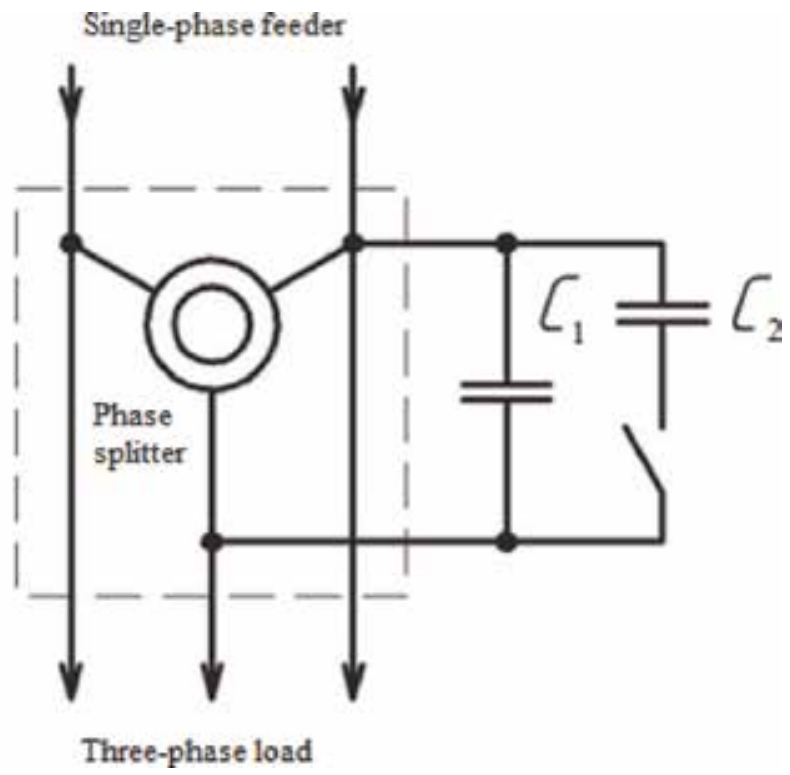

Figure 10.

Schematic diagram of the rotary phase splitter.

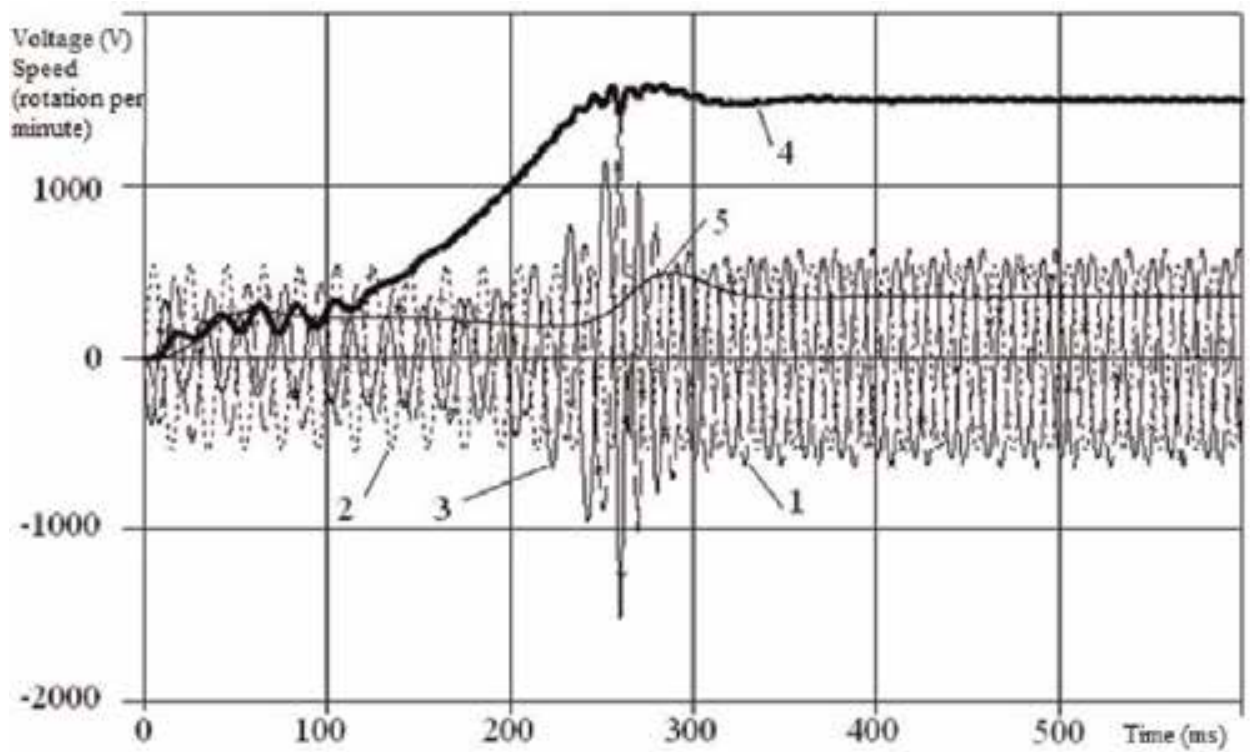

Figure 11.

Results of phase splitter start simulation.

voltage between one of the wires of a single-phase supply and wire of the phase splitter stator winding phase, which is not connected to single-phase supply (see Figure 10), will be over the value of 300 volts-such a situation means the end of the rotor acceleration and ending the formation of a three-phase voltage system (signal 5 in Figure 11).

Figure 12 shows the results of simulation of phase splitting in steady state (at average rotor speed of phase splitter 1500 rotation per minute). In Figure 12 curves 6-8 are the phase currents A, B, and C. Table 1 presents the numerical results at 


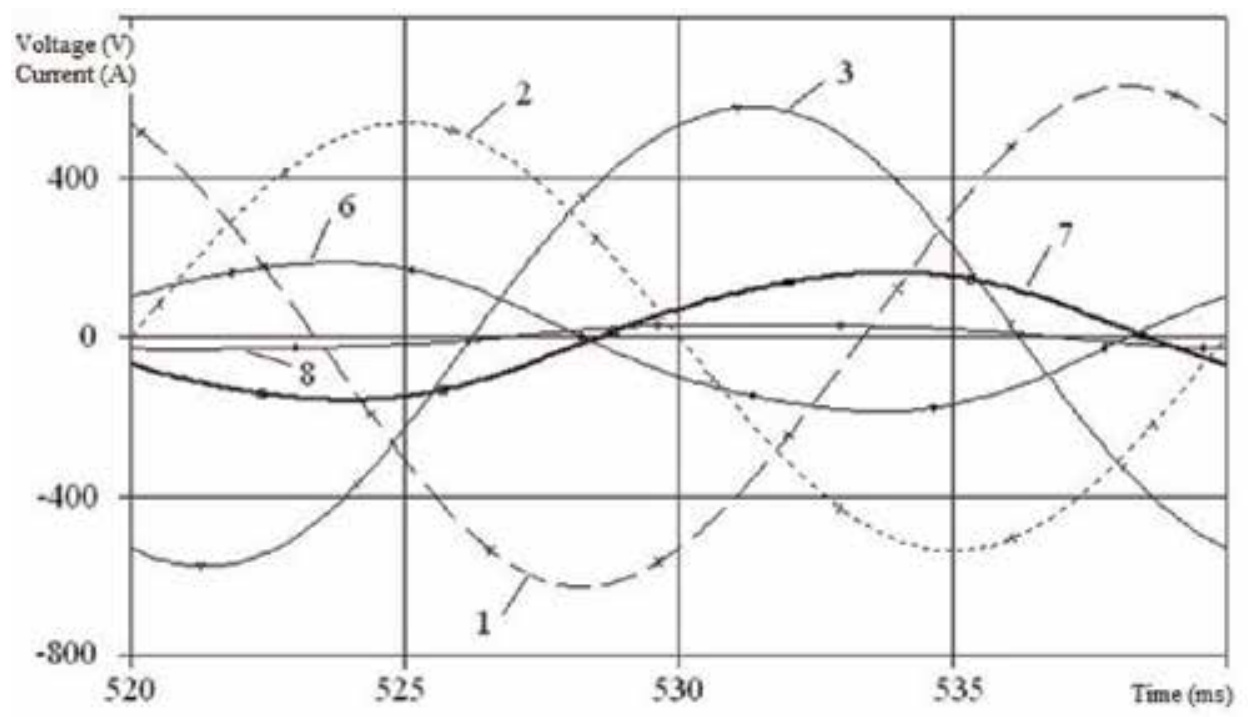

Figure 12.

Results of phase splitter simulation at steady-state mode.

\begin{tabular}{lccccc}
\hline & Phase-to-phase voltages (V) & \multicolumn{4}{c}{ Phase currents (A) } \\
\hline $\boldsymbol{A}-\boldsymbol{B}$ & $\boldsymbol{B}-\boldsymbol{C}$ & $\boldsymbol{C}-\boldsymbol{A}$ & $\boldsymbol{A}$ & $\boldsymbol{B}$ & $\boldsymbol{C}$ \\
\hline 444 & 380 & 408 & 131.9 & 113.9 & 22.1 \\
\hline Splitted & Obtained from a single-phase transformer & Splitted & Without any connected electrical loads \\
& secondary winding & & & & \\
\hline
\end{tabular}

Table 1.

Results of phase splitter simulation at steady-state mode.

steady-state mode. Obviously, the phase splitting effect is clearly demonstrated by the results of simulation.

The proposed mathematical model of a three-phase IM and the method of its computer implementation confirmed their adequacy and effectiveness to address some of the problems of analysis and design of electrotechnical complexes and systems, having IM in its composition.

\section{Conclusion}

In conclusion, the author would like to identify ways of improving the suggested mathematical model of a three-phase IM $[13,14]$. Priority for criticism might be the lack in the mathematical model accounting for the skin effect in the conductors of the rotor winding. In fact, this is a notable disadvantage since in some cases, the current displacement in the rotor is able to accelerate the transitional process of IM start-up in several times $[15,16]$.

There are two types of problems whose solution requires a mathematical model accounting for the skin effect in the conductors of the IM rotor winding: the correct description of transient processes at direct start-up and correct account of the losses in the rotor winding in the case of feeding from the source of non-sinusoidal voltage or current. When deciding tasks of the first type, the author sometimes puts active resistance and leakage inductive resistance of the rotor, which change depending on 
the slip. The solution of tasks of the second type requires the coordination of the complex resistance components of the rotor values to current frequency in it. The second approach is more versatile as it can be used for solution of both types of the abovementioned tasks. According to the author, to implement the second approach, the best way is to create the computer implementation of the mathematical model of a three-phase IM in the original coordinates.

\section{Author details}

Mikhail Pustovetov

Don State Technical University, Rostov-on-Don, Russian Federation

*Address all correspondence to: mgsn2006@rambler.ru

\section{IntechOpen}

(C) 2019 The Author(s). Licensee IntechOpen. This chapter is distributed under the terms of the Creative Commons Attribution License (http://creativecommons.org/licenses/ by/3.0), which permits unrestricted use, distribution, and reproduction in any medium, provided the original work is properly cited. (cc) BY 


\section{References}

[1] Ikeda M, Hiyama T. Simulation studies of the transients of squirrel-cage induction motors. IEEE Transactions on Energy Conversion. 2007;22(2):233-239

[2] Diaz A, Saltares R, Rodriguez C, Nunez R, Ortiz-Rivera E, GonzalezLlorente J. Induction motor equivalent circuit for dynamic simulation. In: Proceedings of the Electric Machines and Drives Conference (IEMDC '09); New York: IEEE International; 2009. pp. 858-863

[3] Sreedevi M, Jeno Paul P. Stator fault detection and diagnosis of an induction motor using neuro fuzzy logic. International Journal of Electrical and Power Engineering. 2011;5(2):102-107

[4] Hung R, Dommel H. Synchronous machine models for simulation of induction motors transients. IEEE Transactions on Power Systems. 1996; 11(2):833-838

[5] Kopylov I. Mathematical Modeling of Electrical Machines. 2nd ed. Moscow: Vyshaya Shkola; 1994. 318 p

[6] Sokola M, Levi E. A novel induction machine model and its application in the development of an advanced vector control scheme. International Journal of Electrical Engineering Education. 2000; 37(3):233-248

[7] Pustovetov M. A universal mathematical model of a three-phase transformer with a single magnetic core. Russian Electrical Engineering. 2015; 86(2):98-101

[8] Keown J. OrCAD PSpice and Circuit Analysis. 4th ed. Upper Saddle River, New Jersey, Columbus, Ohio: Prentice Hall; 2001. 609 p

[9] Halina T, Stalnaya M, Eremochkin S, Ivanov I. Modeling electromechanical characteristics of three-phase motors with inverters vector: Algorithmic type in Matlab Simulink environment. Procedia Engineering. 2016;165: 995-1005

[10] Zirka S, Yu M, Arturi C. Accounting for the influence of the tank walls in the zero-sequence topological model of a three-phase, three-limb transformer. IEEE Transactions on Power Delivery. 2014;29(5):2172-2179

\section{[11] Ogata K. Modern Control}

Engineering. 5th ed. Prentice Hall; 2010. $894 \mathrm{p}$

[12] Arunodai C. Use of Arno converter and motor generator set to convert a single-phase AC supply to a three-phase AC for controlling the speed of a threephase induction motor by using a threephase to three-phase cycloconverter. International Journal of Electrical Engineering \& Technology. 2016;7(2): 19-28

[13] Pustovetov M. A mathematical model of the three-phase induction motor in three-phase stator reference frame describing electromagnetic and electromechanical processes. In: Proceedings of the IEEE Conference Dynamics of Systems, Mechanisms and Machines (Dynamics '16); 15-17 November 2016; Omsk. New York: IEEE; 2016. DOI: 0.1109/ Dynamics.2016.7819069

[14] Pustovetov M. Approach to computer implementation of mathematical model of 3-phase induction motor. In: IOP Conference Series: Materials Science and Engineering 327; 2018. 022085. DOI: 10.1088/1757-899X/327/2/022085

[15] Maksimkina J. Direct start-up of the squirrel-cage induction motor at 
variable parameters of the rotor. Latvian Journal of Physics and Technical

Sciences. 2013;50(1):10-21

[16] Benecke M, Doebbelin R, Griepentrog G, Lindemann A. Skin effect in squirrel cage rotor bars and its consideration in simulation of nonsteady-state operation of induction machines. PIERS Online. 2011;7(5):

421-425 


\title{
CMOS Active Inductor and Its Applications
}

\author{
Dhara Pinkesh Patel
}

\begin{abstract}
Electronic industries always drive to add more functionalities to the devices. Tunability and compactness have become thrust parameters for the microelectronic researchers. In wireless communication, capacitor and inductor are the most significant reactive components for frequency selection. Out of these two reactive components, inductor occupies significant size of entire chip area. As a result, any circuit containing passive inductor such as voltage-controlled oscillator (VCO), low-noise amplifier (LNA), filter, and power dividers consume wider chip size. To meet the requirement of microelectronics industries, passive components have been replaced with active ones. In this chapter, passive inductor has been substituted with CMOS based active inductor.
\end{abstract}

Keywords: active inductor (AI), compact, gyrator, tunability, trans conductance

\section{Passive Inductor}

A passive spiral inductor is an electrical component comprising of coil or wire which is associated with the magnetism and electricity as current passes through the coil. It stores electrical energy in the form of magnetic field. The current passing through the inductor lags inductor voltage by $90^{\circ}$ Figure 1 shows the signal flow diagram of passive inductor.

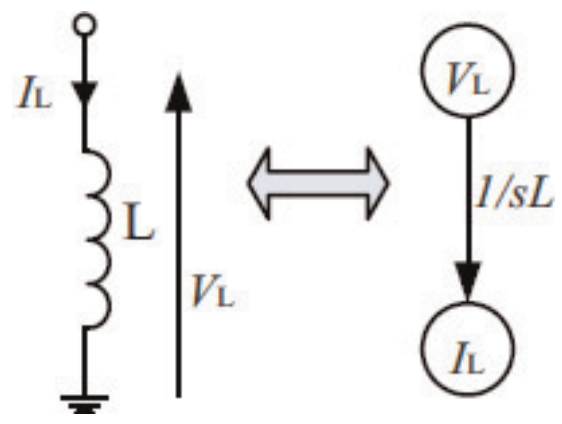

Figure 1.

An ideal spiral inductor and signal flow graph representation. 
Current flowing through the ideal inductor as shown in Figure 1 can be described by the following equation:

$$
\begin{aligned}
& I_{\text {in }}=\left(\frac{1}{s L}\right) V_{\text {in }} \\
& Z_{\text {in }}=\frac{V_{\text {in }}}{I_{\text {in }}}=s L
\end{aligned}
$$

\section{Active inductor}

The design of tunable and compact RF-integrated circuit is challenging. Although spiral inductor is the common implementation approach in integrated circuits, it is possible to design active circuits [1-5]. As reported in [6], active inductor occupies $1-5 \%$ of the passive inductor, and the most unique feature is its tunability of inductance. Further, Table 1 demonstrates the basic comparison between active and passive inductors.

\subsection{Active inductor design methods}

Integrated circuits can be designed for specific frequency bands. Design of active inductors is possible for required operating frequency bands. There are two elementary approaches to configure the active inductors.

1. Operational amplifier-based approach

\section{Gyrator-C-based approach}

The former one is based on operational amplifier (op-Amp), and it is widely used at moderate frequencies (up to about $100 \mathrm{MHz}$ ). The later one is gyrator-C based, which can be operated from sub-gigahertz to gigahertz frequency range. Apart from that, op-amp-based active inductor circuit occupies large chip area and suffers from nonlinearity. As a counterpart, gyrator-based active inductor consumes small chip area and shows better linearity [6]. In present work, to design active inductor at sub $\mathrm{GHz}$, gyrator-C-based active inductor approach has been considered.

\begin{tabular}{lll}
\hline Parameters & Passive inductor & Active inductor \\
\hline Area & Large die area & Small die area \\
\hline Tunability & Fixed & Large tuning range \\
\hline Q factor & Low & High \\
\hline Power consumption & Zero & Significant \\
\hline Noise performance & superior & Poor \\
\hline Linearity & Good & Poor \\
\hline Electromagnetic Interference (EMI) & Significant EMI problems & EMI insensitive \\
\hline
\end{tabular}

Table 1.

Comparison between active and passive inductors. 


\section{Gyrator}

In 1948, scientist of Philips Research Laboratory, Bernard D.H. Tellegen, proposed a new two-port fundamental circuit element. An ideal gyrator is a linear twoport device that couples the current on one port to the voltage on the other port and vice versa. as shown in Figure 2:

$$
\begin{gathered}
i_{1}=G v_{2} \\
i_{2}=-G v_{1}
\end{gathered}
$$

where $G$ presents the gyration conductance.

The gyration conductance $(G)$ relates the voltage on the port $2\left(v_{2}\right)$ to the current in port $1\left(i_{1}\right)$. The voltage on port $1\left(v_{1}\right)$ is associated with current in port 2 $\left(i_{2}\right)$, as minus shows the direction of conductance. It proves that gyrator is a nonreciprocal device. From the gyration conductance, it is called as gyrator [7].

From Eqs. (3) and (4), the ideal gyrator is described by the conductance matrix as shown below,

$$
\left[\begin{array}{l}
i_{1} \\
i_{2}
\end{array}\right]=\left[\begin{array}{cc}
0 & G \\
-G & 0
\end{array}\right]\left[\begin{array}{l}
v_{1} \\
v_{2}
\end{array}\right]
$$

The impedance matrix is defined by:

$$
[Z]=\left[\begin{array}{cc}
0 & R \\
-\boldsymbol{R} & \mathbf{0}
\end{array}\right]
$$

The admittance matrix is expressed by:

$$
[Y]=\left[\begin{array}{cc}
\mathbf{0} & \boldsymbol{G} \\
-\boldsymbol{G} & \mathbf{0}
\end{array}\right]
$$

A generalization of the gyrator is conceivable, in which the forward and backward gyration conductances have different magnitudes, so that the equivalent equations can be written as follows:

$$
\left[\begin{array}{l}
i_{1} \\
i_{2}
\end{array}\right]=\left[\begin{array}{cc}
0 & G_{m 1} \\
-G_{m 2} & 0
\end{array}\right]\left[\begin{array}{l}
v_{1} \\
v_{2}
\end{array}\right]
$$

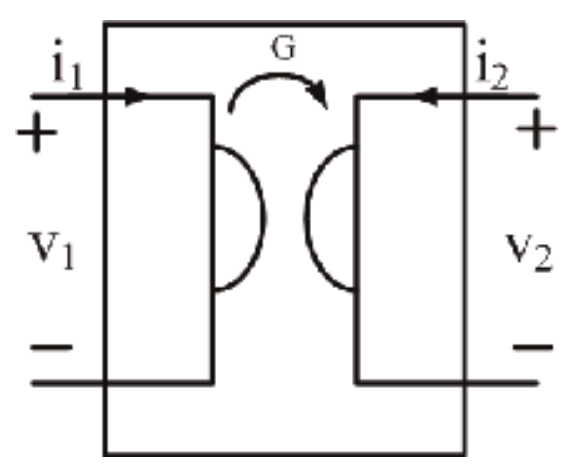

Figure 2.

Ideal gyrator. 


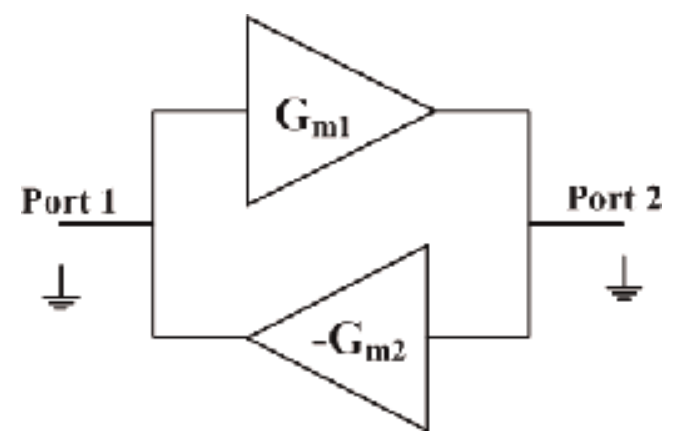

Figure 3.

Ideal gyrator.

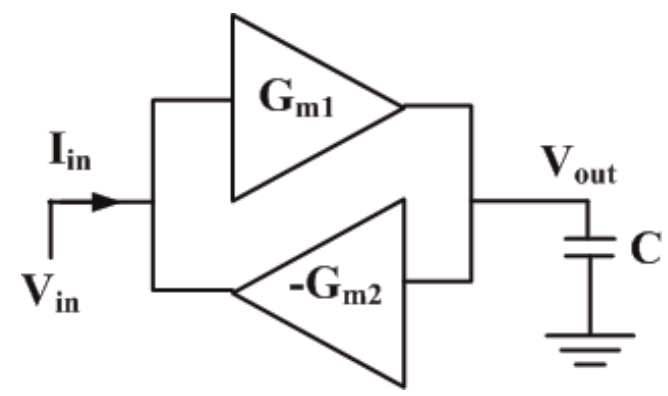

Figure 4.

Ideal gyrator-C based active inductor.

The above matrix can be resulted into block diagram as illustrated in Figure 3. It tells that gyrator comprises of two transconductors: positive transconductor $G_{m 1}$ and negative transconductor $G_{m 2}$, connected in a closed loop as shown in Figure 4. The transcoductor- 1 shows positive transconductance means output current and input voltage are in phase. Whereas, transconductor- 2 depicts negative transconductance means output current and input voltage are $180^{\circ}$ phase shifted (Figure 3 ).

Tellegen noticed that when a capacitor is connected to the secondary terminal (port 2), an inductance is realized at the primary terminal (port 1) of the gyrator, which is entitled as gyrator-C topology as presented in Figure 4.

\section{Gyrator-C-based active inductor and its working principle}

By comparing Eq. (5) with Eq. (2), it clearly tells us that input impedance $Z_{\text {in }}$ is directly proportional to frequency, and the impedance seen at port 2 is inductive. This is an important property of the gyrator, which shows equivalent inductor. Subsequently, equivalent inductance can be defined as,

$$
\begin{gathered}
Z_{\text {in }}=\frac{V_{\text {in }}}{I_{\text {in }}}=\frac{s C}{G_{m 1} G_{m 2}} \\
L=\frac{C}{G_{m 1} G_{m 2}}
\end{gathered}
$$

Therefore, gyrator-C network is used to synthesize active inductor. This synthesized inductor is called as gyrator- $\mathrm{C}$ active inductor. The inductance of active 
(a)

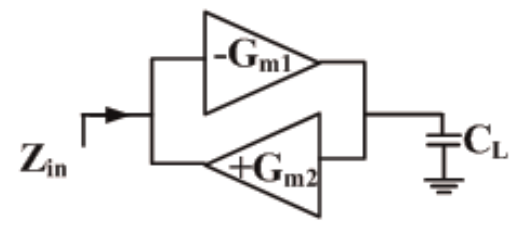

(b)

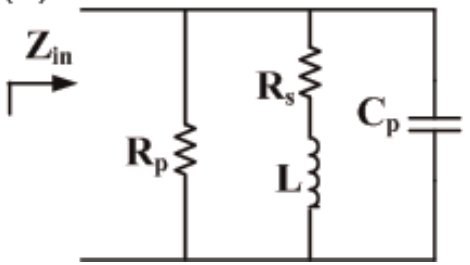

Figure 5.

Practical gyrator-C-based inductor (a) block diagram, (b) equivalent circuit diagram.

inductor is proportional to the load capacitance $\mathrm{C}$ and inversely proportional to the product of the transconductances of the transconductors.

In practical active inductor circuit, along with the inductance, we do get parasitic components as series resistance $R_{s}$, parallel resistance $R_{p}$ and parallel capacitance $C_{p}$. These parasitic RC components ultimately affecting on the performance of active inductor (Figure 5).

\section{Performance parameters of active inductors}

In order to define the active inductor circuit, few parameters need to be measured. In this section, we shall discuss about the several active inductor parameters such as inductive range, tunability, quality factor, power consumption, linearity, supply voltage sensitivity and noise. These analyses can be understood when we go through the ac analysis, noise analysis, transient analysis and harmonic analysis.

\subsection{Inductive tuning range}

An ideal gyrator-C-based active inductor shows inductive behaviour over the entire frequency range. But a lossy active inductor exhibits resistive, inductive and capacitive frequency range. The respective range can be analysed by analysing the impedance of equivalent RLC circuit of active inductor. In order to acquire the relation between frequency and magnitude, bode plot has been used. Further to accomplish the bode plot of frequency versus impedance magnitude, $1 \mathrm{~A}$ sinusoidal ac current is applied at input port. As a result, the voltage measurement at the same input port results into input impedance. Below mentioned steps are required to derive the tuning inductive range.

1. To derive the inductive band frequency for active inductor, we need to draw the small signal model of the circuit. After that application of Kirchoff's voltage or current law helps to simplify the circuit. The input impedance of equivalent circuit of practical active inductor can be written as shown in Eq. (1).

$$
Z_{\text {in }}=\left(\frac{R_{s}}{C_{p} L}\right) \frac{s \frac{L}{R_{s}+1}}{s^{2}+s\left(\frac{1}{R_{p} C_{p}}+\frac{R_{s}}{L}\right)+\frac{R_{s}+R_{p}}{R_{p} C_{p} L}}
$$

2. The inductive pole frequency of $Z_{\text {in }}$ is given by 


$$
\omega_{p}=\sqrt{\frac{R_{p}+R_{s}}{R_{p} C_{p} L}}
$$

Because $R_{p} \gg R_{s}$, it is simplified as,

$$
\omega_{p} \approx \sqrt{\frac{1}{L C_{p}}}=\omega_{0}
$$

where $\omega_{0}$ is the maximum inductive frequency of the active inductor. Above Eq. (8) tells us that the higher value of inductance and parallel capacitance enhance the value of resonance frequency.

3. The zero frequency can be written as,

$$
\omega_{z}=\frac{R_{s}}{L}
$$

Eq. (10) mentions that zero frequency is proportional to series resistance and inversely proportional to inductance.

4. From the zero and pole frequency, the inductive frequency range can be defined as,

$$
\omega_{z}=\frac{R_{s}}{L} \leq \omega \leq \frac{1}{\sqrt{L C_{p}}}=\omega_{0}
$$

Eq. (11) clearly depicts that $R_{s}$ affects the lower range of the inductive frequency range, whereas the upper range of inductive frequency can be set by maximum inductive frequency of the active inductor. In order to achieve maximum inductive range, both the $R_{s}$ and $C_{p}$ should be minimum at given inductance $\mathrm{L}$.

5. To verify the inductive behaviour, the phase response is equally important.

The entire inductive range impedance phase should be $90^{\circ}$. In other words, voltage should lead the current by $90^{\circ}$.

Higher number of transistors in gyrator active inductor generates the parasitic poles, which increases the phase shift of transistors and reduces the inductive tuning range [7].

To observe the inductive behaviour through the bode plot, we can apply $1 \mathrm{~V} \mathrm{ac}$ current to the input node and observe the voltage at output node. Subsequently, frequency response gives the frequency versus impedance magnitude plot, and the phase plot can give the frequency versus phase. Figure 6 shows the bode plot response on Cadence Spectre Spice tool. The inductive frequency can be defined at which the phase is $90^{\circ}$.

\subsection{Inductance tunability}

The active inductor can be tuned by two different ways:

1. Variation in the transconductance of the transconductors

2. Variation in the load capacitance (Figure 7) 


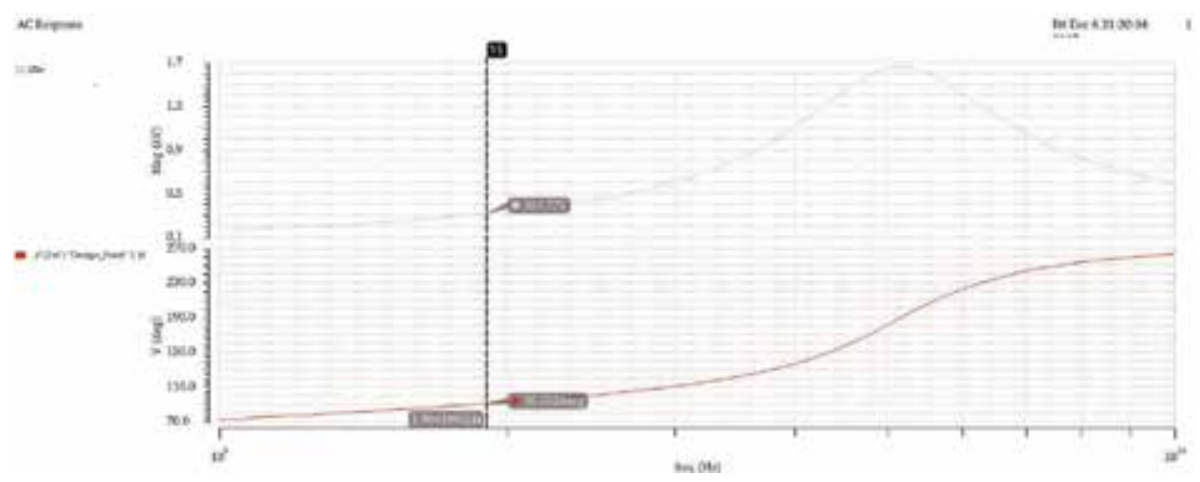

Figure 6.

Bode plot of CMOS active inductor [1].

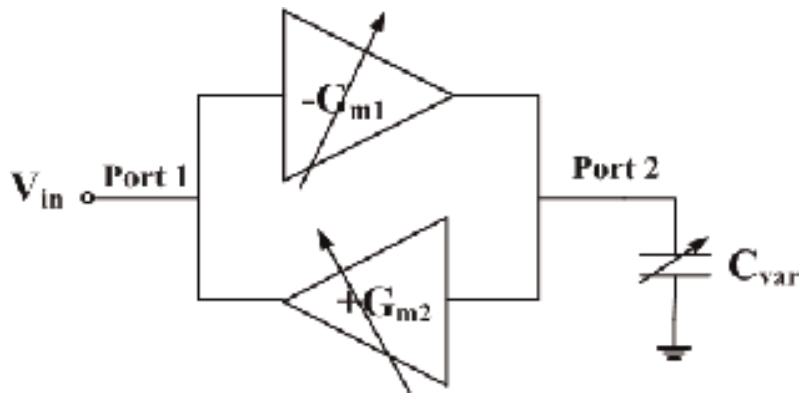

Figure 7.

Tuning in gyrator-C network.

\subsection{Quality factor}

Spiral inductor contains a small coil resistance in addition to inductance, and hence, it has quite low quality factor. The quality of inductance in coil is defined by quality factor. It is defined as the ratio of reactance of the coil to its series resistance.

$$
Q=\frac{X_{L}}{R}
$$

For CMOS active inductor, once the input impedance is derived, then quality factor can be defined as,

$$
Q=\frac{\operatorname{Im}\left(Z_{\text {in }}\right)}{\operatorname{Re}\left(Z_{\text {in }}\right)}
$$

From Eqs. (7) and (13), quality factor of active inductor can be represented as,

$$
Q=\left(\frac{\omega L}{R_{s}}\right) \frac{R_{p}}{R_{p}+R_{s}\left[1+\left(\frac{\omega L}{R_{s}}\right)^{2}\right]}\left[1-\frac{R_{s}^{2} C_{p}}{L}-\omega^{2} L C_{p}\right]
$$

The higher value of quality factor shows the low loss in active inductor. It is a crucial parameter to design active inductor. The higher and sustainable quality factor over the entire inductive frequency range is the challenge for analogintegrated circuit designers. In depicted paper [1], Widlar current source has been used to enhance the quality factor of inductor and in [2], it has been used in voltagecontrolled oscillator. 


\subsection{Noise}

As active inductor is made up of active components, it suffers from the internal noise. The main noises can be listed as thermal noise, flicker noise and noise due to the distributed substrate used in transistors. Through the equivalent noise model of active inductor circuit, we can analyze it clearly.

\subsection{Linearity}

For ideal active inductor, all the transistors of circuit must be in saturation region over the entire inductive tuning range. But, in practical active inductor circuit, few transistors switch over from saturation to triode region. The transconductance of transistor varies from saturation to triode region. As a result, the inductance value also gets changed, which adds nonlinearity in the active inductor working. Active inductor linearity can be characterized by two different ways, total harmonic distortion (THD) and $1 \mathrm{~dB}$ inductance compression $L_{-1 d B}$. THD exhibits the ratio of total unwanted harmonic current to fundamental current when a fundamental ac input signal has been injected into the active inductor. $L_{-1 d B}$ can be defined as the input current amplitude has become $1 \mathrm{~dB}$ large than its small signal value.

\subsection{Power consumption}

Active components in active inductor consume static power. Less number of transistors in submicron CMOS technology and circuit configuration without body effect are generally used methods to reduce power consumption in active inductor.

\subsection{Supply voltage sensitivity}

The fluctuations in DC supply voltage of active inductor also affect the performance of active inductor.

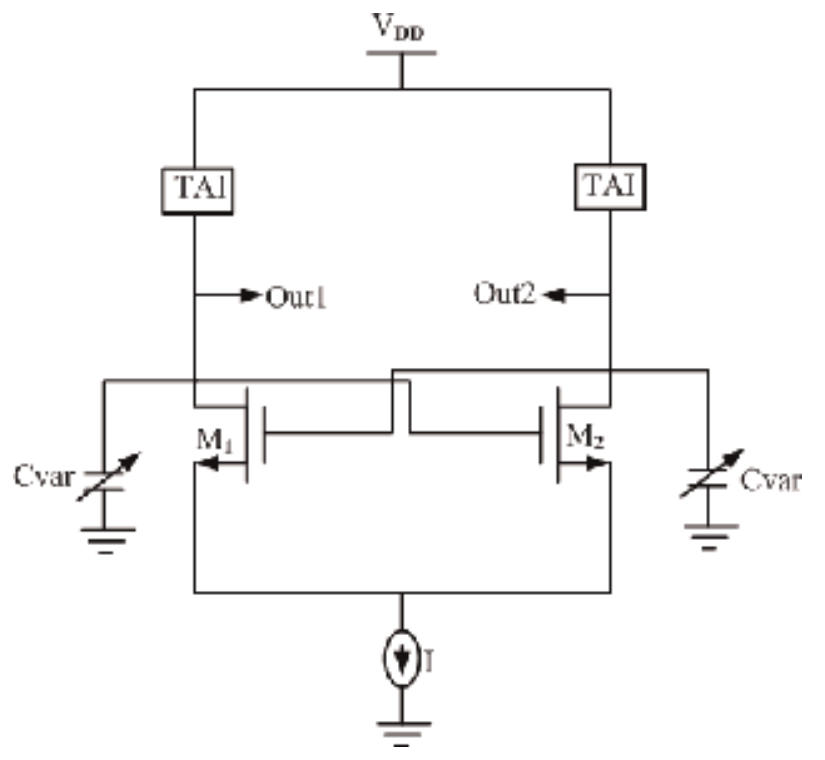

Figure 8.

Tunable voltage-controlled oscillator. 


\section{Applications}

\subsection{Active inductor-based voltage-controlled oscillator}

The performance of RF front-end mainly relies on the performance of the individual RF blocks. Mostly, in each block, inductor has been used such as in low noise amplifier, filter, matching circuit, voltage-controlled oscillator, power divider, etc. For frequency selection, reactive components as inductor and capacitor play crucial role. Out of these two reactive components, inductor occupies significant size of entire chip area. Figure 8 depicts the active voltage-controlled oscillator where the passive inductor has been replaced with tunable active inductors (TAI).

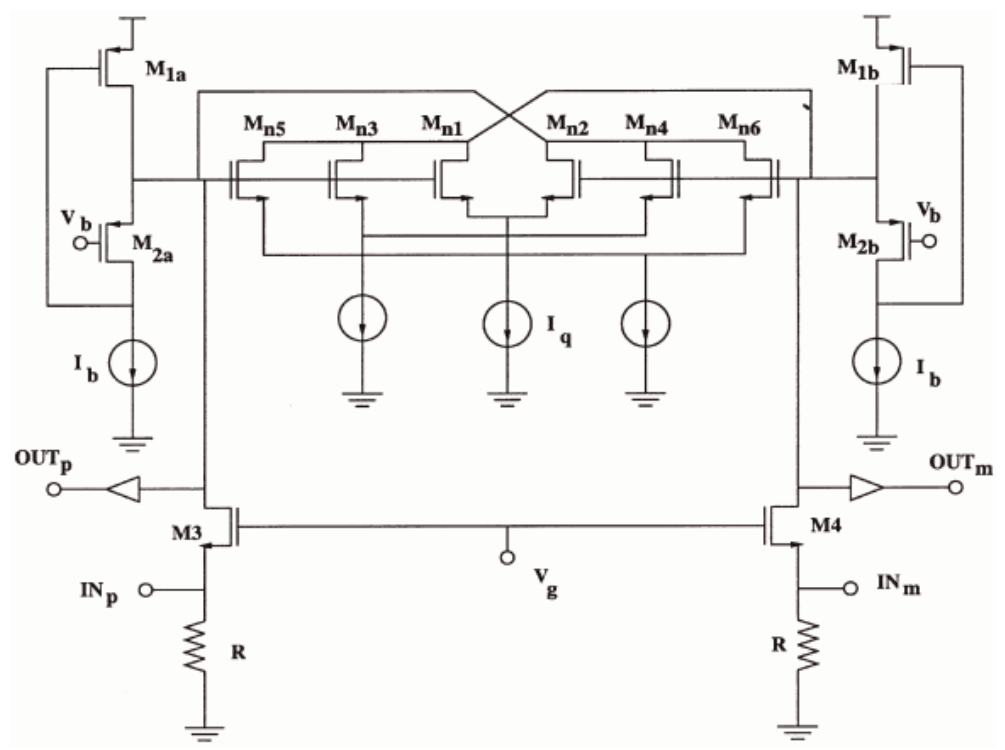

Figure 9.

Active inductor based filter [8].

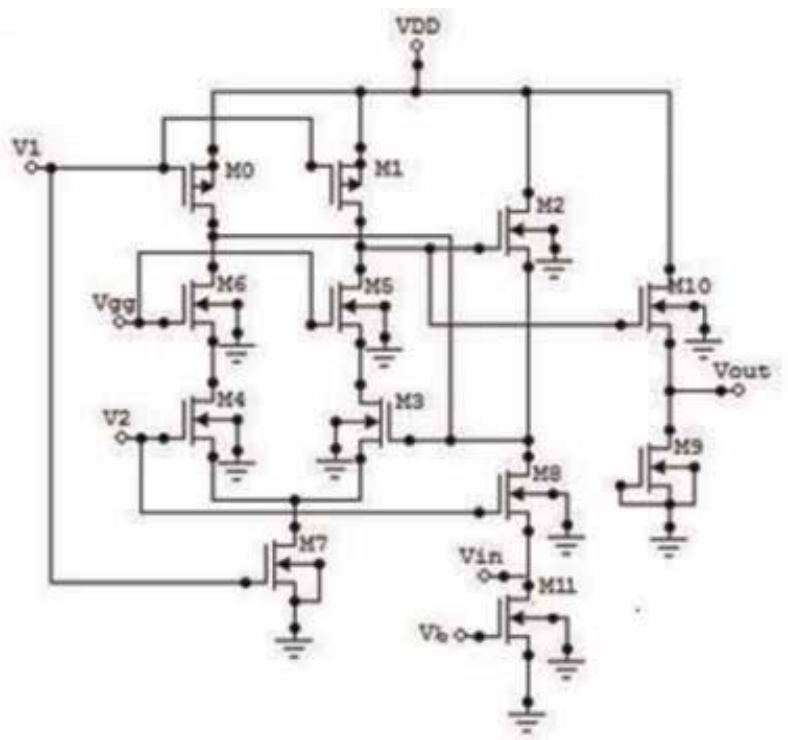

Figure 10.

Active inductor-based low noise amplifier [9]. 
Generally, voltage-controlled oscillator is tuned using varactor capacitor where the tuning range is limited. Using tunable active inductor, we get more freedom to tune it over wider frequency range. Subsequently, we can operate more standards using a single VCO. Furthermore, it consumes less chip area.

\subsection{Active inductor-based filter}

In [8], a second-order RF bandpass filter based on active inductor has been implemented in a $0.35 \mathrm{~m}$ CMOS process. The operating frequency of the active filter is $900 \mathrm{MHz}$ with the quality factor of 40 (Figure 9).

\subsection{Active inductor-based low noise amplifier}

In [9], low noise amplifier has been designed using active inductor at the ISM frequency of $2.4 \mathrm{GHz}$ (Figure 10).

\section{Conclusions}

The designing of active and tunable electronic components is the emerging field of today's microelectronics industry. To facilitate the compact and efficient chips, we must have to use them in our applications like voltage-controlled oscillator, filter and low noise amplifier. Apart from these, inductor has been used in matching circuits, power combiners and power dividers. Moreover, it can be used in input circuits to control the loading effects. In all these applications, passive inductor has been replaced with active inductor, where we can benefit in compactness and tenability. The more other parameters are discussed in chapter.

\section{Conflict of interest}

The author has no conflict of interest to declare.

\section{Notes/thanks/other declarations}

I hereby declare that this chapter on "CMOS Active Inductor and Its Applications" was carried out by me for the book publication on "Electromagnetic Devices and Machines." 


\section{Author details}

Dhara Pinkesh Patel

V.T. Patel Department of Electronics and Communication Engineering, Faculty of Technology and Engineering, Chandubhai S. Patel Institute of Technology, Charotar University of Science and Technology, Gujarat

*Address all correspondence to: dharapatel.ec@charusat.ac.in

\section{IntechOpen}

(C) 2019 The Author(s). Licensee IntechOpen. This chapter is distributed under the terms of the Creative Commons Attribution License (http://creativecommons.org/licenses/ by/3.0), which permits unrestricted use, distribution, and reproduction in any medium, provided the original work is properly cited. (c) BY 


\section{References}

[1] Patel DP, Oza-Rahurkar S. Tunable CMOS active inductor using widlar current source. Journal of Circuits, Systems and Computers. 2019;28(2): 1950027. DOI: $10.1142 /$

S0218126619500270

[2] Patel DP, Oza-Rahurkar S. CMOS active inductor/resonator based voltage controlled oscillator. Recent Advances in Electrical and Electronic Engineering. 2019;12(1). DOI: $10.2174 / 23520$ 96511666181105111852

[3] Patel DP, Oza-Rahurkar S. CMOS active inductor: A technical review. International Journal of Applied Engineering Research. 2018;13(11): 9680-9685. ISSN 0973-4562

[4] Razavi B. Design of CMOS Integrated Circuits. California,USA: McGraw Hill; 2001. ISBN: 0-07-238032-2

[5] Yuan F. CMOS Active Inductors and Transformers. 1st ed. Ontario, Canada: Springer; 2008. ISBN: 978-0-387-76477-1

[6] Tellegen BDH. Passive Four Terminal Network for Gyrating a Current into a Voltage. Google Patents 2,647,239. 1953. Available from: https://patents.google.com/patent/ US2647239

[7] Xiao H, Schaumann B. High Frequency Active Inductor. US Patent 7,042,317 B2. 2006. Available from: https://patents.google.com/pate nt/US7042317B2/en

[8] William B. Kuhn. Dynamic range performance of on-chip rf bandpass filters. IEEE Transactions on circuits and systems-II: Analog and digital signal processing. October 2003;50(10)

[9] Arif Sobhan Bhuiyan M. Design of an active inductor based LNA in $130 \mathrm{~nm}$ CMOS process technology. Journal of Microelectronics. 2015;45(3):188-194 


\title{
Low-Cost (PM Less) Wide-Speed-Range-Operation Generators
}

\author{
Sorin Ioan Deaconu, Feifei Bu, Marcel Topor, \\ Lucian Nicolae Tutelea and Nicolae Muntean
}

\begin{abstract}
This chapter presents a novel dual stator-winding induction generator (DSWIG) system for wind power generation, and an optimal scheme to decrease the capacity of static excitation converter (SEC) is also given. The main result is represented by the finding that reactive excitation power released by the excitation capacitor and SEC is not only correlated to generator parameters, speed range, and load but also affected by wind turbine power curve. This chapter also investigates the optimal excitation capacitor selection process. Considering the objective of minimizing the capacity of SEC, several methods are tested here to identify an appropriate excitation capacitor value. Using the general d-q model in the stator-voltageorientation synchronous frame of the DSWIG control algorithm and model of SEC, a decoupling control strategy using the space vector modulation (SVM) is determined for the six-phase DSWIG. Based on the obtained models, the computer simulation and experimental investigations of a test prototype orated at $18 \mathrm{~kW}$ with six stator phases and three-phase wound rotor DSWIG wind power system were carried out to validate the optimal solution for the system The matching results (simulation and teststand measurements) demonstrate the correctness and effectiveness of this optimization scheme.
\end{abstract}

Keywords: DSWIG, excitation capacitor, static excitation controller, space vector modulation

\section{Introduction}

In the latest 30 years, due to the depletion of fossil fuels and the increasing environmental concerns and consequently strict regulations, wind energy has become one of the most widely integrated renewable resources, attracting more and more investments and scientific attention. Statistically by the year of 2012, about $282,430 \mathrm{MW}$ of wind power were installed all over the world [1], and it is targeted that in 2030 a percent equal to $10-20 \%$ of the global electrical energy used will be produced by wind farms [2]. As a result of the general orientation toward a higher degree of wind energy generation in the grid, there is a strong direction to install the wind farms offshore. The reason is represented by the fact that the wind resource has a better availability and the problems of noise pollution and the effect on the site can be handled without great difficulty [3]. 
The large offshore wind farms will require a transmission of energy produced from the offshore wind farm to the grid connection point. The general solution is to have a high-voltage ac (HVAC) or high-voltage dc (HVDC) transmission. Usually, offshore wind farms are located at distances $<120 \mathrm{~km}$ from the shore. This wind farms have the capacity $<150 \mathrm{MW}$ which is possible to be connected to the grid using an HVAC transmission, whereas at longer distances and/or with higher capacity, only the HVDC transmission is recommended mainly due of its economic and technical advantages [4].

There are a large number of topologies of electric machines which can be can be used as generators for wind power systems, among which we may include the synchronous machine, induction machine, switched reluctance machine, etc. $[5,6]$. However, the very strict requirements to reach high performance and high reliability are the development of the large and long-distance offshore wind farms operating with the HVDC transmission. In order to have this, a number of technical proposals have been considered to be promising [5, 7-10]. The large majority of them are built on the permanent magnet synchronous generator (PMSG) topology and on the doubly fed induction generator (DFIG) topology. It is considered that the PMSG-based generator wind turbines feeding the load through a dc bus voltage is appropriate for the HVDC transmission between the farm and shore grid, Even though this type of generators has a higher power density and efficiency, they have a cost disadvantage given to the rapid increase of rare-earth material price. This rather simple solution requests a full-rated power converter which is disequilibrium in the cost of installment and operation between the generator and the power converter [9]. In contrast to the DFIG, it requires power converter representing only $25 \%$ of the generator rated power (if we consider a limited speed range of 0.75 1.25 p.u.). Of course the presence of slip rings and brushes in order to supply power to the rotor side leads to a rise of the maintenance cost. If we consider a HVDC transmission, a voltage-source converter, or a line-commutated converter, it has to be included since the voltage output of the DFIG is three-phase constant ac voltage and frequency [10].

\section{State of the art in dual stator-winding induction generators (DSWIG)}

The rotor cage-type induction generator (IG) constitutes an excellent choice for building wind turbine generators. It has many advantages for wind power applications, such as natural brushless construction, low maintenance request, good overload protection capability, and many other excellent features [11]. For traditional IG systems, one important disadvantage is the difficulty to realize regulation of excitation reactive power and the poor output voltage dynamic performance under the variations of load and speed, which limits their extended applications. With the rapid development of power electronics and electric machine control strategies, many improved topologies have been proposed to circumvent the aforementioned problems [11-14]. We can mention here at least one which is represented by the connection of a dc-ac voltage-source inverter in parallel or series with the loads to provide variable excitation reactive power. This solution has facilitated a performant control of IG systems, but most of these improved solutions will require a certain amount of injected harmonics into the load current and will have as side effect induced the output voltage ripple.

A step forward into the problem to effectively control the output voltage and eliminate/decrease the harmonics generated from power commutation, a dual stator-winding induction generator (DSWIG) with a static excitation converter (SEC) as proposed in [15]. Under the variable speed and load regime in order to get constant frequency and constant voltage, this system suffers from the complexity or 
low efficiency due to the use of a discharge resistance or battery [16]. For power systems operating in aircrafts, ships, and wind power applications, a DSWIG-based dc generating system with one or more diode rectifiers connected to the power winding is recently explored in [16-19]. This kind of system is characterized by a good performance of the dc voltage output with a small-size power converter capable of operating under the dynamic variable speed and load, which indicates the fact that it can also be used in wind power systems, especially as a suitable solution for the offshore wind farms using the HVDC transmission technology.

The prime mover in wind power system is the wind turbine. The output power of it is not only related to the wind speed but also has much to do with the rotor speed, turbine speed, rotor blade tilt, rotor blade pitch angle size and shape of turbine, swept area of turbine blades, rotor geometry whether it is a HAWT or a VAWT, and wind speed turbulences which constitute the mathematical model of the wind turbine. The characteristics of the wind turbine depend on the type of prime mover. The turbine position and its nonlinearities should be taken into consideration seriously when designing or controlling a wind power system. Nevertheless, the long list of research papers written for the type of DSWIG is concentrated primarily upon the control and optimization for the abovementioned power system configuration. When the DSWIG system is powered by the wind turbine, if we consider the capacity optimization problem for SEC, in our opinion these available optimal schemes mentioned in literature are not appropriate for the DSWIG used in wind power farm.

As a consequence of the existing constraints regarding the overall cost and volume, the capacity of the chosen SEC is one of the important elements of the design of wind power system. In this chapter, the optimal sizing algorithm of excitation capacitor designed to decrease the capacity of SEC for DSWIG wind power system is presented. We have considered a number of methods to be investigated and employed to identify the optimal excitation capacitor in a step-by-step procedure. The simulation and experimental results from the tests performed on $18 \mathrm{~kW}$ six-phase/three-phase DSWIG wind power system prototype supplying a rectifier load for dc voltage output are shown for comparison and validation.

\section{System topology and model}

\subsection{DSWIG wind power system}

The configuration of a prototype six-phase/three-phase DSWIG wind power system is shown in Figure 1.

The stator winding consists in a two set of shifted windings which are located in the stator slots in this generator. The first winding referred to as ix-phase power winding, is built using two three-phase star windings shifted by a 30-degree electric angle, and supplies power to the dc load via a six-phase bridge rectifier. The second winding, identified as a three-phase control winding, is connected to the static excitation controller (SEC). A standard squirrel-cage rotor is used by this generator. The two windings (power and control) have the same number of poles, so they share the same working frequency. It has been found that [1] because there is no electric connection between the two sets of windings, the electromagnetic compatibility (EMC) of the system is improved greatly.

Wind energy is transformed into mechanical energy and therefore into dc power by the wind turbine and DSWIG, respectively. The power winding will transfer the generated power to the dc load through the rectifiers connected at the grid side. Using two three-phase diode bridges connected in parallel or series, it is possible to supply a dc bus or a dc load. 


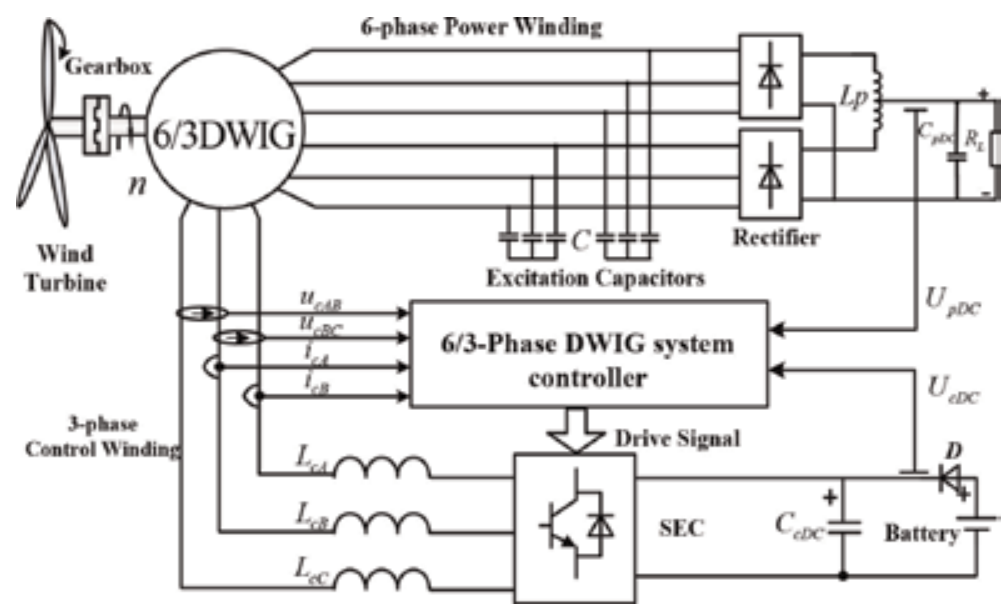

Figure 1.

Six-phase/three-phase DSWIG wind power system.

In this chapter, we have considered that the parallel connection of the windings with an interphase reactor is selected. In order to provide partial reactive power for the excitation of the generator, a fixed excitation capacitors bank to the terminal of six-phase power winding. In case of the control winding, a switch-mode pulsewidth modulation (PWM) voltage-source SEC is used to produce variable reactive power to secure the performance of dc voltage output according to the different working conditions. In this configuration, the dc bus capacitor behaves as a voltage source of SEC.

In this rather simple electric power generation system, the prime mover is the wind turbine. As we may know, the output mechanical energy of the wind turbine varies with the wind and rotor speed besides many other factors. According to the Betz law, the rotor speed should be constantly dynamically adapted with the wind to capture more wind power, which can be implemented by the control of output power $[20,21]$. As a consequence the system should naturally have the capability to self-adapt to the variations of speed and load, which will conduct to a large change of the reactive excitation power produced by the fixed excitation capacitors and to a great variation of the capacity of SEC. However, there is a possible solution if the excitation capacitors connected to the power winding were optimally selected. In this case the capacity of SEC will be decreased considerably, which will lead to a low-cost power and compact-size generation system. Therefore, the optimal algorithm for the selection of the excitation capacitor is very important for the DSWIG applied in wind power system.

In this type of system, the objective is to have the dc bus voltage of power winding $U_{p \text { DC }}$ constant. In order to have this condition fulfilled, the control objectives need to consider the speed and load variations. This implies that we need to maintain $U_{c D C}$, the dc bus voltage of SEC, stable as this is the basic condition for the normal work of SEC. According to the instantaneous power theory, it is possible to control both the active and reactive components of the control winding current separately, and in order to maintain a direct control of the generator, instantaneous active and reactive power is used in order to meet the control objectives [22]. In this chapter, we present a mathematical model of SEC developed using the d-q frame with the reference orientation being synchronous to the stator voltage. In this work, the closed interconnection between the $\mathrm{d}$ and $\mathrm{q}$ axis quantities is considered, and based on this, the rather new control strategy with decoupling using SVM for the DSWIG wind power generator is introduced. 


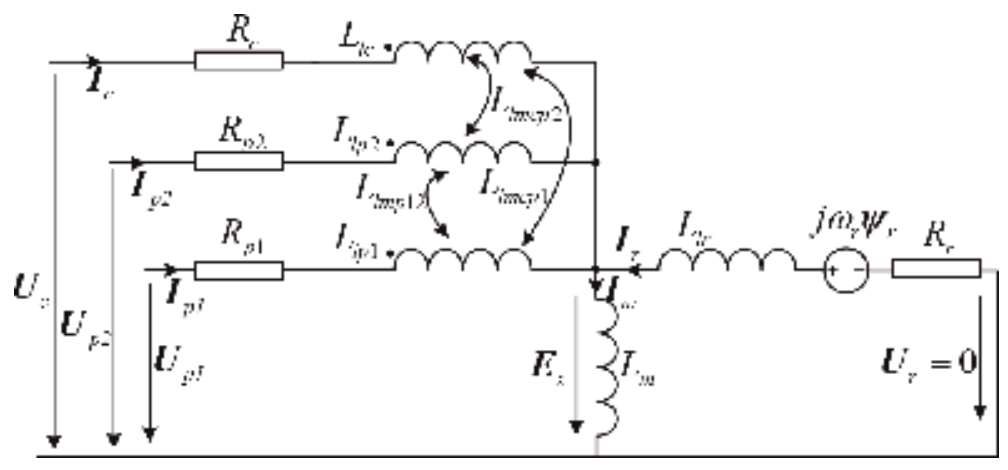

Figure 2.

The equivalent circuit of six-phase/three-phase DSWIG in stationary reference frame.

\subsection{DSWIG model}

The equivalent circuit schematic of six-phase/three-phase DSWIG in the stationary reference frame is shown in Figure 2. In this representation, the equivalent circuit parameters of the rotor winding and the control winding are referred to the power winding. The dynamic model of the generator expressed in the complex variable form can be written as follows [15]:

The voltage equations are written as

$$
\left\{\begin{array}{l}
\boldsymbol{U}_{p 1}=R_{p 1} \boldsymbol{I}_{p 1}+d \boldsymbol{\psi}_{p 1} / d t \\
\boldsymbol{U}_{p 2}=R_{p 2} \boldsymbol{I}_{p 2}+d \boldsymbol{\psi}_{p 2} / d t \\
\boldsymbol{U}_{c}=R_{c} \boldsymbol{I}_{c}+d \boldsymbol{\psi}_{c} / d t \\
\boldsymbol{U}_{r}=R_{r} \boldsymbol{I}_{r}-j \omega_{r} \boldsymbol{\psi}_{r}+d \boldsymbol{\psi}_{r} / d t
\end{array}\right.
$$

where

$$
\left\{\begin{array}{l}
\boldsymbol{\psi}_{p 1}=L_{p 1} \boldsymbol{I}_{p 1}+L_{l m p 12} \boldsymbol{I}_{p 2}+L_{l m c p 1} \boldsymbol{I}_{c}+L_{m} \boldsymbol{I}_{r} \\
\boldsymbol{\psi}_{p 2}=L_{l m p 12} \boldsymbol{I}_{p 1}+L_{p 2} \boldsymbol{I}_{p 2}+L_{l m c p 2} \boldsymbol{I}_{c}+L_{m} \boldsymbol{I}_{r} \\
\boldsymbol{\psi}_{c}=L_{l m c p 1} \boldsymbol{I}_{p 1}+L_{l m c p 2} \boldsymbol{I}_{p 2}+L_{c} \boldsymbol{I}_{c}+L_{m} \boldsymbol{I}_{r} \\
\boldsymbol{\psi}_{r}=L_{m} \boldsymbol{I}_{p 1}+L_{m} \boldsymbol{I}_{p 2}+L_{m} \boldsymbol{I}_{c}+L_{r} \boldsymbol{I}_{r}
\end{array}\right.
$$

$L_{c}=L_{m}+L_{l c} ; L_{r}=L_{m}+L_{l r} ; L_{p 1}=L_{p 2}=L_{m}+L_{l p} ; L_{l p}, L_{l c}$, and $L_{l r}$ represent the leakage inductance corresponding to the power winding, the control winding, and, respectively, the rotor; $L_{l m}$ represents the mutual leakage inductance between the stator windings; and $L_{m}$ is the magnetizing inductance. The variables $\boldsymbol{U}, \boldsymbol{I}$, and $\boldsymbol{\psi}$ represent the voltage, current, and flux; their subscript letters $p, c$, and $r$ indicate the correspondence to the stator power winding, stator control winding, and rotor. Numbers (1) and (2) indicate the first and second set of power winding distinctly. The derivative of the above variables is written by $d / d t$, and $\omega_{r}$ is the angular rotor electrical frequency.

\section{Principle of design with optimization}

\subsection{Wind turbine characteristics}

The power captured by the wind turbine is expressed by Eq. (3), which is a function depending on the blade shape, the pitch angle, the radius, and the rotor speed of rotation [20]: 


$$
P_{T}=\frac{1}{2} \pi \rho R^{2} C_{p}(\lambda, \beta) V^{3}
$$

where $\rho$ is the air density (in $\mathrm{kg} / \mathrm{m}^{3}$ ), $R$ is the blade radius (in $\mathrm{m}$ ), $\beta$ is the pitch angle (in degrees), $C_{p}(\lambda, \beta)$ is the wind turbine power coefficient, and $V$ is the wind speed (in $\mathrm{m} / \mathrm{s}$ ). $\lambda$ is the tip-speed ratio, defined as

$$
\lambda=\frac{2 \pi n R}{60 V}
$$

where $n$ is the rotor speed (in rev/min).

The general wind turbine power curves for various wind speeds are shown in Figure 3. From this it can be easily noticed that, for each wind speed, there exists a maximum power point in the wind turbine output. This point is located on the power versus rotating speed characteristic. $P_{\text {opt }}$, in Figure 3 the line connecting this point, is the maximum power curve which is the line coupling all the maximum power points obtained at different wind speeds.

The maximum power point is obtained for the wind turbine having a fixed pitch angle (the $\beta$ is fixed), when the tip-speed ratio $\lambda$ equals $\lambda_{\text {opt }}$, and the wind turbine power coefficient $C_{p}$ is equal to maximum. In this condition, the maximum output power can be written as

$$
\begin{gathered}
P_{T \max }=\frac{1}{2} \pi \rho R^{2}\left(\frac{2 \pi R}{60 \lambda_{\text {opt }}}\right)^{3} C_{p \max } n^{3} \\
\lambda_{\text {opt }}=\frac{2 \pi R}{60} \cdot \frac{n_{\text {opt }}}{V}
\end{gathered}
$$

One may need to maximize the output power of wind turbine within the range of variation of wind speed. For this reason the rotor speed must be kept proportional with the wind speed to insure the value of the tip-speed ratio constant for all the maximum power point according to Eqs. (5) and (6). The control of the generator requires the use of the maximum power point tracking (MPPT) method for the wind turbine operating at a variable speed. The process can be implemented in advanced controller in order to strength optimal power generation output, like in $[20,21]$.

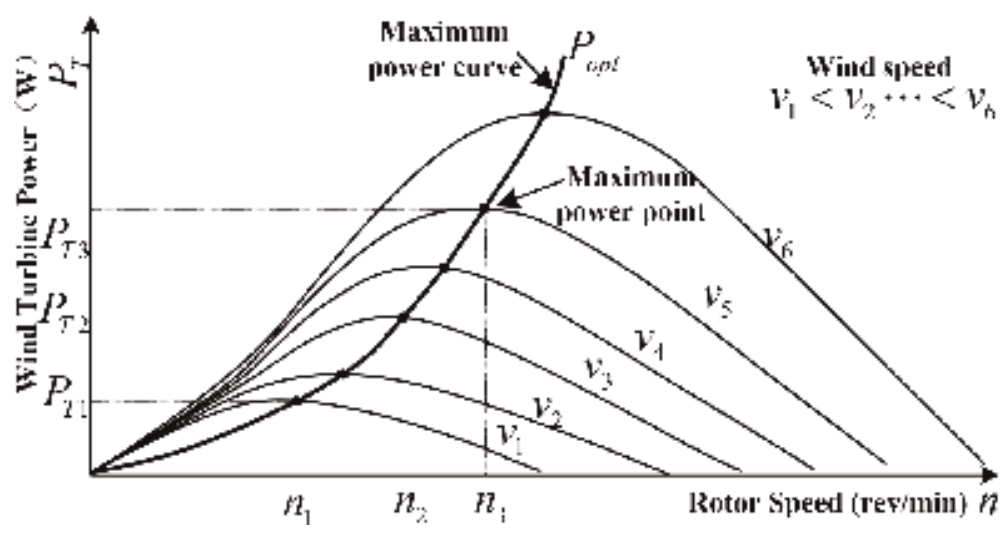

Figure 3.

Wind turbine power curves for various wind speeds. 


\subsection{The optimal selection algorithm of excitation capacitor}

Considering the various wind speed and wind turbine power curve areas, the operation of DSWIG wind power system can be divided into four stages A-B-C-DE, as shown in Figure 4.

\subsubsection{Stage I: segment A-B: the initialization sequence of wind power system}

The process initiates when the wind speed is greater than the start-up value. At this moment the rotor blades initiate the spinning, and the rotor speed slowly increases, as shown in A-B segment in Figure 4. The generator will not provide any power during this sequence.

\subsubsection{Stage II: segment B-C: the voltage buildup sequence of DSWIG wind power system}

In this stage, the system is initiating the voltage generation when the minimum speed need is obtained. During this sequence, the reactive power needed for excitation is supplied through the excitation capacitor and SEC simultaneously. In addition to this, it is necessary to maintain the dc-side capacitor voltage constant with the SEC supplying the part of the active power required for charging, which is essential to the normal operation of the system [22]. The generating mode is possible after the nominal excitation voltage is reached.

\subsubsection{Stage III: segment C-D: the operation of system within the range of low wind speed}

When the cut-in speed is reached, the generator begins to produce enough power in order to begin the export. The rotor speed is still low due to the small wind speed. As a result, the output voltage of rectifier connected to the power winding cannot reach the nominal value. One may notice that the output power is related to the cube of the wind speed, and, thus, it is very small in this wind speed operating zone. In order to take advantage of this part of the wind curve characteristic, we consider the voltage pump-up theory as it is presented in [23]. When the voltage in the DC bus of SEC is reaching the reference value, the output power is mainly generated through the SEC from the control winding. The matching equations of wind speed, rotor speed, and output power are represented under the form of a curved line labeled B-C in Figure 4.

\subsubsection{Stage IV: segment D-E: the large wind speed domain regime of the wind power system}

When the value of speed of the wind system is relatively stable within the large wind region, the rectified output voltage can match the nominal value, and the

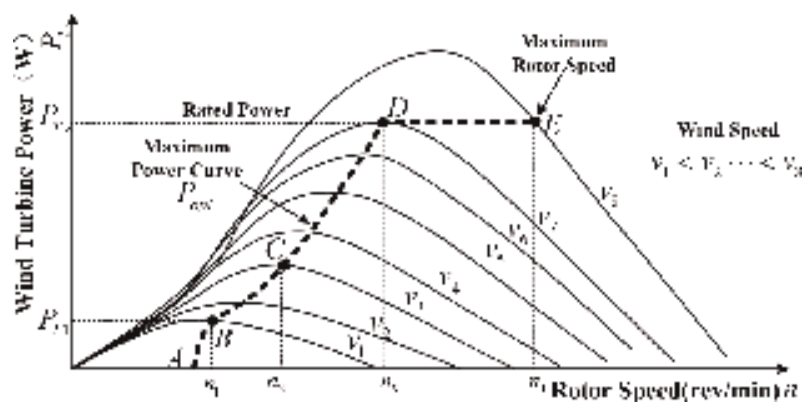

Figure 4 .

The characteristic of DSWIG wind power system variable wind speed. 


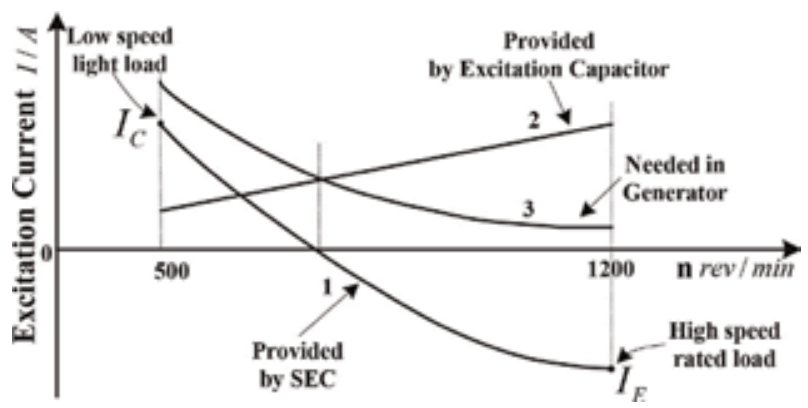

Figure 5 .

The change of excitation current in CDE segment in large wind speed zone.

wind power generator system can provide energy at maximum power under different wind speeds (i.e., $P_{\text {opt }}$ in Figure 4). Since constant output voltage is needed, the reactive power needed for generator excitation provided by the excitation capacitors is not sufficient, and for this reason, the remaining share is given by the SEC. When the wind is accelerating steadily, the wind turbine speeds up, so the reactive excitation power provided by the excitation capacitor also increases; thus, the reactive energy supplied by the SEC decreases accordingly. During the speed up, the SEC gets more reactive excitation power from the generator instead of providing it. If the wind speed slows down beyond the rated value, some correcting actions need to be performed in order to limit the output power of the wind turbine [10]. A first measure is the modification of the pitch angle. Another measure is to reduce the magnetization field. The field should be weakened in order to be sure that the constant output power of the generator is kept. This process is represented by the CDE segment in Figure 4 where the change of excitation current is exposed in Figure 5.

Curve 1 in Figure 5 presents the control winding current variation when the generator operates within the range of CDE area. When the current value is positive, the SEC provides insufficient reactive power to the generator; and when the value is negative, the SEC there is an excessive reactive excitation which imposes a correction. The maximum positive current $I_{C}$ in control winding occurs at lowspeed, light-load situations (as it can be noticed from Figure 5) (corresponding to point $C$ in Figure 4), while the negative minimal current $I_{E}$ occurs at high-speed rated-load moments (corresponding to point $E$ in Figure 4).

In this chapter, the sizing equations based on mathematical model and simulation methods are used to obtain an suitable the excitation capacitor value, capable of maintaining the current flowing through the control winding at a variable speed and load regime following strictly the law, $\left|I_{C}\right|=\left|I_{E}\right|$. Thus, the minimal capacity of SEC is found for the DSWIG wind power system.

\subsection{The selection of optimal excitation capacitor for an $18 \mathrm{~kW}$ six-phase/three-phase DSWIG wind power system}

\subsubsection{Optimal excitation capacitor identification using the graphic method}

The equivalent circuit parameters of the prototype $18 \mathrm{~kW}$ multiphase stator windings with six-phase/three-phase DSWIG studied in this paper are given in Appendix. The waveforms of the control winding current $I_{c}$ obtained using various excitation capacitors are analyzed, and the results are shown in Figure 6. In this figure, two different situations regarding the low-speed, light-load and the high- 
speed rated-load moments were investigated. The shape of curve $I_{c 1}$ synthesizes the effect the control winding current at $500 \mathrm{rev} / \mathrm{min}$ with $4 \mathrm{~kW}$ (the corresponding maximum output power to this speed). Curve $I_{c 2}$ illustrates the effect of the control winding current at $1200 \mathrm{rev} / \mathrm{min}$ with $18 \mathrm{~kW}$. Output of the excitation capacitor value ranges from $10 \mu \mathrm{F}$ to $140 \mu \mathrm{F}$.

Curve $-I_{c 2}$ is the symmetrical about the $\mathrm{X}$-axis with curve corresponding the $I_{c 2}$. The maximum absolute value of the control winding positive and negative current is equal in this process, and thus the intersection of curve $I_{c 1}$ and $-I_{c 2}$ is point $\mathrm{O}$, which is indicated that the excitation capacitor is $70 \mu \mathrm{F}$. In few words, the optimal excitation capacitor can be easily determined by this graphic method.

\subsubsection{Optimal value of the excitation capacitor identification using the iterative approach}

Using a value $70 \mu \mathrm{F}$ as the optimal excitation capacitor, the variation of control winding current is rendered under the form of curve 1 in Figure 7. In this graphic, the maximum positive current is $I_{C}=8 \mathrm{~A}$ and the minimal negative current is

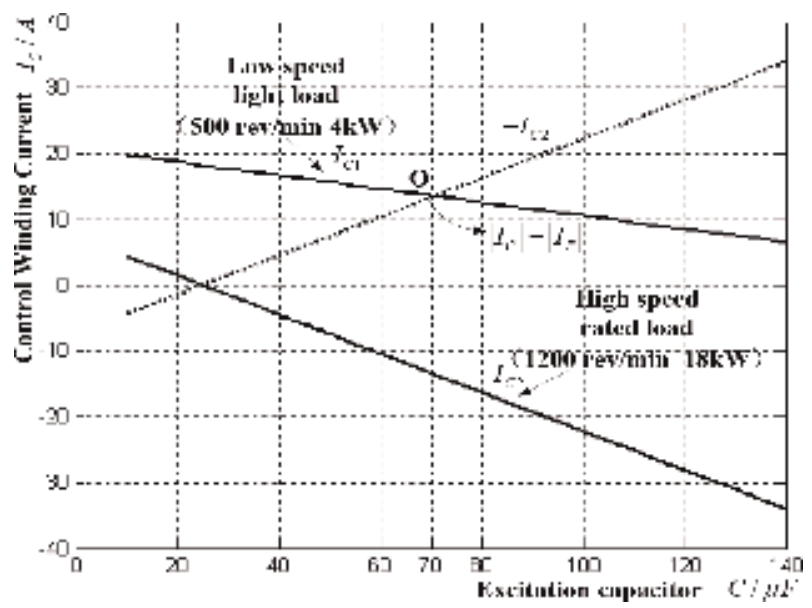

Figure 6.

The influence of various excitation capacitors on the control winding current.

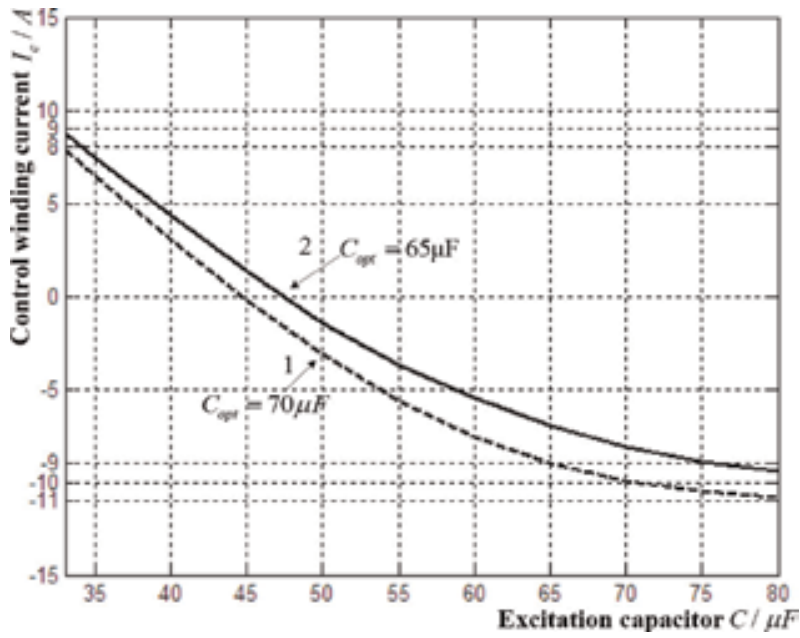

Figure 7.

The law of control winding current based on the optimal excitation capacitor. 


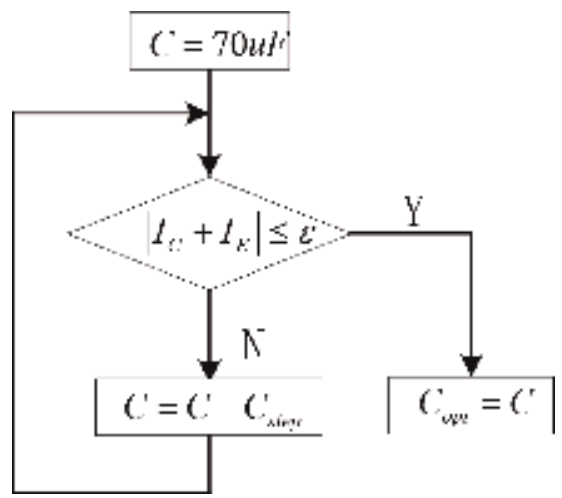

Figure 8.

The sketch map of iterative algorithm.

$I_{E}=-10 \mathrm{~A}$. This value implies that the excitation capacitor is somewhat large, requiring a reduction of the value.

The graphic method is based on the identification of the two curves intersection graphically, where the intersection denotes the optimal excitation capacitor. Although some errors are expected, the result can be considered as a reference value. Therefore, this value can be established as the initial guess value for the recursive method. In this chapter, in order to identify the optimum value recursive algorithm, the capacitance value of the excitation capacitor is viewed as an optimization variable, $\left|I_{C}+I_{E}\right| \leq \varepsilon$ as the optimization constrain target, and the initial guess of the capacitance equal to $70 \mu \mathrm{F}$ is the starting initial value.

The recursive algorithm flowchart is shown in Figure 8. The optimal value is obtained after several iterations, and after the target reaches the optimal excitation capacitor, $C_{o p t}=65 \mu F$ is obtained. Considering this as the optimal value of the excitation capacitor, we obtain curve 2 in Figure 7 is accomplished. Through the entire optimization process, the absolute values of maximum and minimum currents flowing through the control winding are both about $9 \mathrm{~A}$. In fact, $I_{c}$ echoes the capability of SEC to provide excitation for the generator. By means of a synthesis of graphical and recursive methods, the optimal value for excitation capacitor can be found to apprehend the capacitor value minimization of SEC for the wind power system with DSWIG generator connected to a passive resistance load.

\section{Control strategies}

\subsection{A synthetic analysis of the control algorithms for the multiphase DSWIG system}

According to the analysis depicted in Ref. [22], the two sets of windings share the same air-gap flux; thus any change of control winding flux will cause the variation of air-gap flux, which can determine a reaction in the DC-bus voltage of power winding $U_{p \mathrm{DC}}$. Due to the power loss occurring inside the SEC, $U_{c D C}$ would be faded. In this way, $U_{c D C}$ must be strictly controlled to enforce the standard work condition of SEC, which can be further realized by the controller of the electromagnetic torque produced by control winding $T_{e m c}$. The relationship between corresponding parameters can be expressed as follows: 


$$
\left\{\begin{array}{l}
\psi_{c} \rightarrow U_{p D C} \\
T_{e m c} \rightarrow U_{c D C}
\end{array}\right.
$$

Based on the instantaneous power theory [24], the control winding instantaneous power can be stated as in Eq. (8) in the d-q coordinate frame referencing the control winding terminal voltage vector $U_{c}$ :

$$
\left\{\begin{array}{l}
p_{c}=\omega_{1} \psi_{c} i_{c d} \\
q_{c}=\omega_{1} \psi_{c} i_{c q}
\end{array}\right.
$$

This way we can obtain the following equality:

$$
\frac{p_{c}}{q_{c}}=\frac{i_{c d}}{i_{c q}}
$$

where $p_{c}$ and $q_{c}$ denote the instantaneous active and reactive power generated by the current in the control winding, respectively. $i_{c d}$ and $i_{c q}$ are the d-q components of control winding current $i_{c} . \omega_{1}$ is the angular stator electrical frequency.

Eq. (8) shows that the q component of control winding current, $i_{c q}$, is generating the instantaneous reactive power $q_{c}$, while $i_{c d}$ generates $p_{c}$, namely:

$$
\left\{\begin{aligned}
i_{c d} & \rightarrow p_{c} \\
i_{c q} & \rightarrow q_{c}
\end{aligned}\right.
$$

From equation [22], in variable speed and load regime, the management of $T_{e m c}$ can be realized by the control of two components, namely $p_{c}$ and $q_{c}$, which can finetune the control winding flux. Briefly, the following relationship can be obtained:

$$
\left\{\begin{array}{l}
p_{c} \rightarrow T_{e m c} \\
q_{c} \rightarrow \psi_{c}
\end{array}\right.
$$

As it is stated above, the control algorithms for the DSWIG power generation system functioning in variable rotor speed and load regime driven by wind power turbine may be written as it follows according to Eqs. (7), (10), and (11):

$$
i_{c} \stackrel{U_{c} \text {-oriented }}{\longrightarrow}\left\{\begin{array}{l}
i_{c d} \rightarrow p_{c} \rightarrow T_{e m c} \rightarrow U_{c D C} \\
i_{c q} \rightarrow q_{c} \rightarrow \psi_{c} \rightarrow U_{p D C}
\end{array}\right.
$$

\subsection{The mathematical equivalent model for the DSWIG system}

In order to simplify the equations, only the fundamental component of voltage is considered, and the hysteresis and eddy current losses are disregarded. Using this assumptions DSWIG can be considered as a voltage source seen from the side of the control winding [25]. Figure 9 shows the equivalent circuit of the DSWIG system in simplified vector form.

Using the aforementioned equations, the voltage equation of the equivalent circuit can be yielded as

$$
\boldsymbol{U}_{m}=L \frac{d \boldsymbol{I}_{c}}{d t}+\boldsymbol{U}_{c}
$$

Eq. (13) can be rewritten using the $\alpha-\beta$ reference frame: 


$$
\left[\begin{array}{c}
U_{m \alpha} \\
U_{m \beta}
\end{array}\right]=L \frac{d}{d t}\left[\begin{array}{l}
i_{c \alpha} \\
i_{c \beta}
\end{array}\right]+\left[\begin{array}{l}
U_{c \alpha} \\
U_{c \beta}
\end{array}\right]
$$

If the $\mathrm{d}$-q coordinates is referenced to the control winding terminal voltage vector $\boldsymbol{U}_{c}$, shown in Figure 10, Eq. (14) can be transformed into the d-q reference frame coordinates:

$$
\left[\begin{array}{l}
U_{m d} \\
U_{m q}
\end{array}\right]=L \frac{d}{d t}\left[\begin{array}{l}
i_{c d} \\
i_{c q}
\end{array}\right]+L \omega_{1}\left[\begin{array}{l}
-i_{c q} \\
i_{c d}
\end{array}\right]+\left[\begin{array}{l}
U_{c d} \\
U_{c q}
\end{array}\right]
$$

where

$$
\left[\begin{array}{l}
U_{c d} \\
U_{c q}
\end{array}\right]=\left[\begin{array}{l}
U_{c} \\
0
\end{array}\right]
$$

where $U_{c}$ is the magnitude of $U_{c}$, and Eq. (17) can be obtained by the joint of Eqs. (15) and (16):

$$
\left[\begin{array}{l}
U_{m d} \\
U_{m q}
\end{array}\right]=L \frac{d}{d t}\left[\begin{array}{l}
i_{c d} \\
i_{c q}
\end{array}\right]+L \omega_{1}\left[\begin{array}{l}
-i_{c q} \\
i_{c d}
\end{array}\right]+\left[\begin{array}{l}
U_{c} \\
0
\end{array}\right]
$$

In this way, the control winding current in the $\boldsymbol{U}_{\boldsymbol{c}}$-oriented d-q coordinates can be written as

$$
\left\{\begin{array}{l}
i_{c d}=\int \frac{U_{m d}+L \omega_{1} i_{c q}-U_{c}}{L} d t \\
i_{c q}=\int \frac{U_{m q}+L \omega_{1} i_{c d}}{L} d t
\end{array}\right.
$$

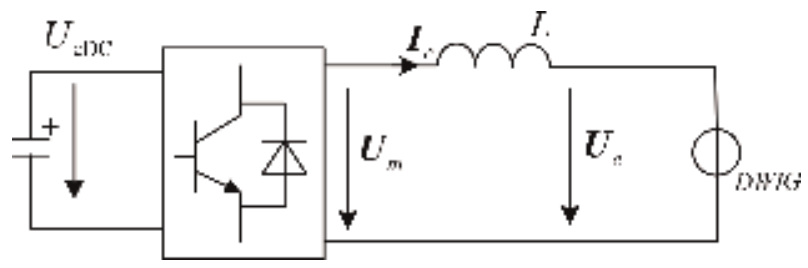

Figure 9.

The equivalent circuit of the DSWIG system.

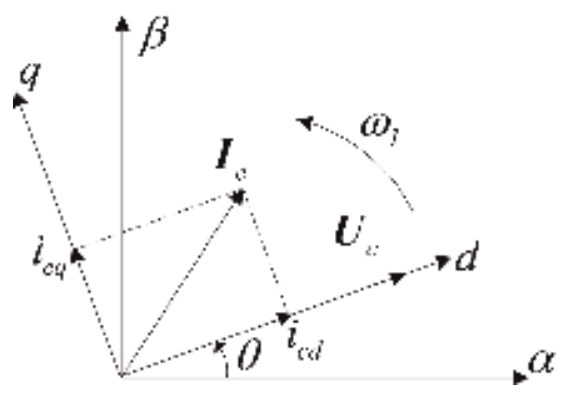

Figure 10.

The stator voltage orientation of the control winding. 
Based on the analysis presented previously, we may develop the mathematical equivalent model of control winding connected with SEC. This model is specified in Figure 11. The d-q components of $\boldsymbol{I}_{c}, i_{c d}$, and $i_{c q}$ will control the instantaneous active and reactive power, respectively.

This controller can be implemented by usually making use of PI-type current controller to make the control winding current track the command current. However, Eq. (18) implies that there is a cross coupling between the $\mathrm{d}$ and q channels, which obviously if we consider Figure 11. This cross coupling will decline the static and dynamic performance.

\subsection{Decoupling control strategy using space vector modulation SVM}

A decoupling control strategy using SVM for the DSWIG wind power system is presented in Figure 12.

The command voltage components are provided given as function depending on the two-axis PI regulators output and the inductance:

$$
\left\{\begin{array}{l}
U_{m d}^{* d e}=P I\left(i_{c d}, i_{c d}^{*}\right)-L \omega_{1} i_{c q}+U_{c} \\
U_{m q}^{* d e}=\operatorname{PI}\left(i_{c q}, i_{c q}^{*}\right)+L \omega_{1} i_{c d}
\end{array}\right.
$$

where $U_{m d}^{* d e}$ and $U_{m q}^{* d e}$ are used for the SEC by the SVM; Eq. (19) can be converted into the following equation:

$$
\left\{\begin{array}{l}
P I\left(i_{c d}, i_{c d}^{*}\right)-L \omega_{1} i_{c q}+U_{c}=L \frac{d i_{c d}}{d t}-L \omega_{1} i_{c q}+U_{c} \\
P I\left(i_{c q}, i_{c q}^{*}\right)+L \omega_{1} i_{c d}=L \frac{d i_{c q}}{d t}+L \omega_{1} i_{c d}
\end{array}\right.
$$

This expression can simplify into an integral form:

$$
\left\{\begin{array}{l}
i_{c d}=\int \frac{P I\left(i_{c d}, i_{c d}^{*}\right)}{L} d t \\
i_{c q}=\int \frac{P I\left(i_{c q}, i_{c q}^{*}\right)}{L} d t
\end{array}\right.
$$

Equation (21) implies that it is possible to linearize the relationship between the input and output of the model of control winding including SEC using the

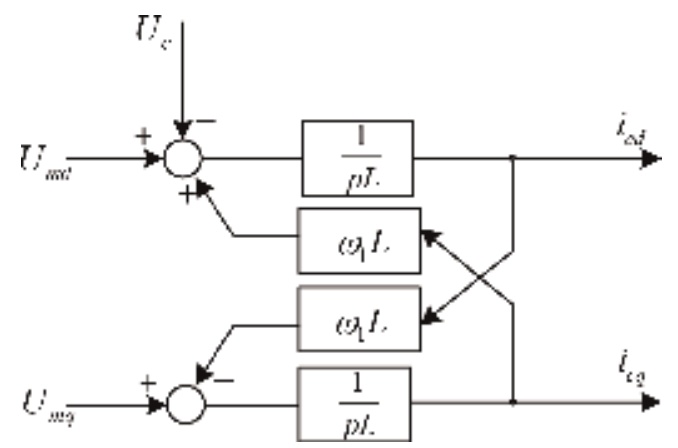

Figure 11.

The model of control winding including SEC. 


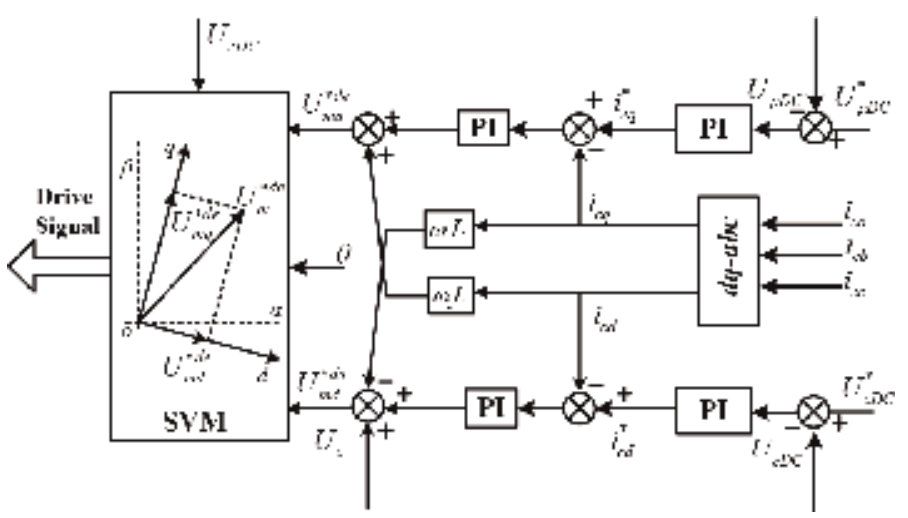

Figure 12.

The decoupling control strategy using SVM for six-phase/three-phase DSWIG system.

command voltage with corresponding decoupling elements. This way, the system naturally guarantees that the decoupling control strategy is effective transforming the equations into a first-order linear dynamic system. Using the command voltage $U_{m d}^{* d e}$ and $U_{m q}^{* d e}$, the SVM is employed to generate the switching signals for the power switches.

\section{Simulation and experimental results}

\subsection{Simulation results}

Theoretically, the optimal excitation capacitor obtained from the sizing algorithms corresponds to a generator loaded only with a resistive load, excluding the influence of rectifier load. But in practice the nonlinear influence of rectifier load for the whole system cannot be fully neglected.

The simulation model for six-phase/three-phase DSWIG wind power generation system is built based on the decoupling control strategy using SVM presented above. The equations were coded into a complex model with the help of MATLAB/ Simulink to additionally fine-tune the value of the optimal excitation capacitor.

In this simulation model, the suitable tunings for $C_{\text {opt }}$ are made, when $C_{\text {opt }}=62 \mu \mathrm{F}$. The simulation results are shown in Figure 13.

When the rotor speed is inferior to the value of $300 \mathrm{rev} / \mathrm{min}$, there is no voltage at the output of the generator, as shown in phase 1 in Figure 13. If the speed is growing over $300 \mathrm{rev} / \mathrm{min}$, the system begins to build up the voltage. From phase 2 in Figure 13, it can be noticed that the corresponding dc bus voltage $U_{p D C}$ and $U_{c D C}$ will raise progressively with the development of line-to-line voltage and line current in the control winding. As a consequence of the low speed, the voltage in the dc bus output of power winding $U_{p D C}$ cannot increase and maintain itself up to the specified value $600 \mathrm{~V}$. On the contrary, the $U_{c D C}$ can yield $600 \mathrm{~V}$ due to the voltage pumping-up capability of control winding. The output power is small in phase 3 in Figure 13 as it may be seen in the power curve. Consequently, the power is provided from control winding side somewhat from the power winding over SEC. With the additional growth of the rotor speed, the dc voltage of power windingrectified output can arise up to $600 \mathrm{~V}$ of the specified value of the generation system. At this situation, the state change of this DSWIG system is engaged. The control winding output generated power is surpassed by the power output of the 
power winding. In phase 4 in Figure 13, this changeover is exposed. The modification of $U_{p D C}$ raising from 200 to $600 \mathrm{~V}$ can be seen, while $U_{c D C}$ is preserved at $600 \mathrm{~V}$ constantly.

After this transient state, the rotor speed ramps up from 500 to $1200 \mathrm{rev} / \mathrm{min}$ and stays at $1200 \mathrm{rev} / \mathrm{min}$ afterward, as shown in phase 5 in Figure 13. During this process, while we have an increase of the rotor speed and load corresponding to the

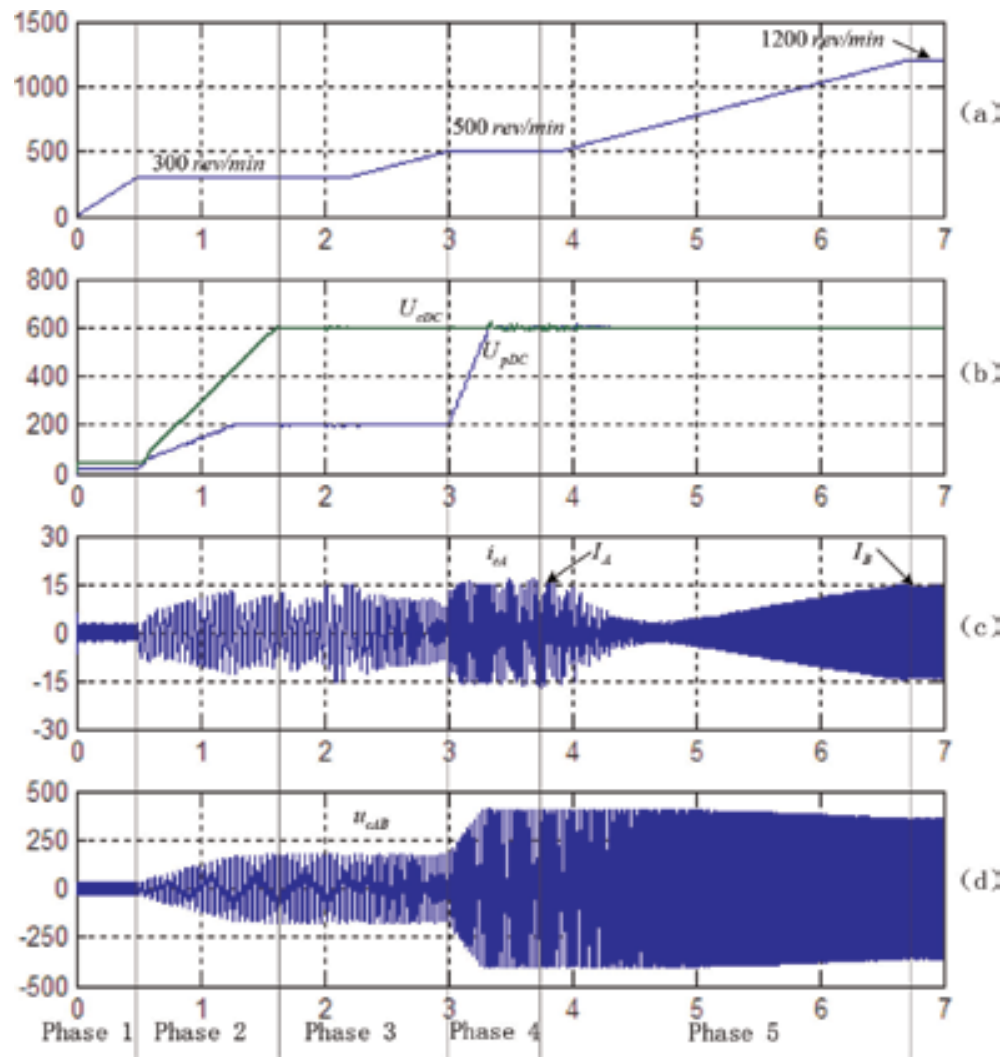

Figure 13.

Simulation results of six-phase/three-phase DSWIG wind power system based on the optimal capacitor $(62 \mu F)$. (a) The rotor speed $n$. (b) The dc bus voltage $U_{c D C}$ and $U_{p D C}$. (c) The line current of control winding $i_{c A}$. (d) The line-to-line voltage of control winding $u_{c A B}$.

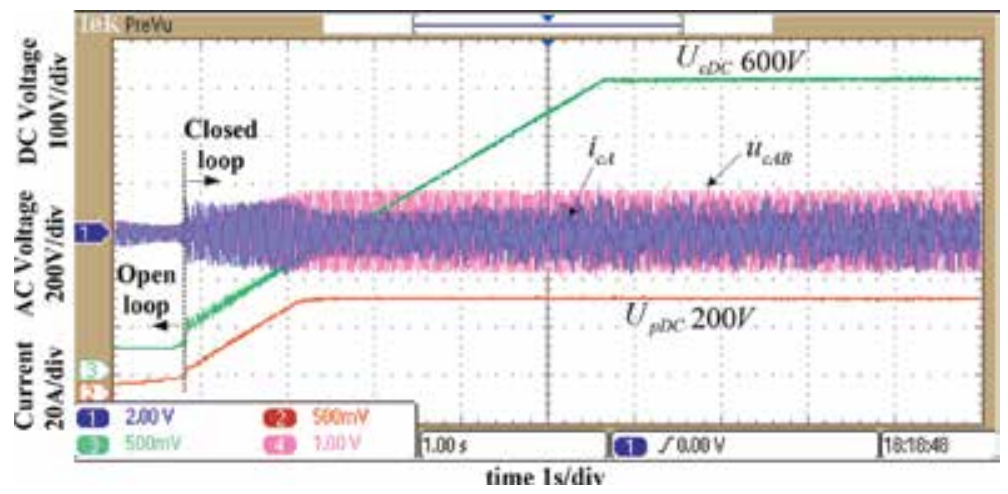

Figure 14.

Experimental waveform of the DSWIG wind power system voltage buildup. 
power curve, the control winding line current $i_{c A}$ rms value shifts from negative to positive slope, the control winding current at low speed with light load (500 rev/ min $4 \mathrm{~kW}$ ) becomes equal with the value obtained during high speed with rated load regime (1200 rev/min $18 \mathrm{~kW}$ ), which obeys to the equation $\left|I_{C}\right|=\left|I_{E}\right|$, and the rms value is about $10 \mathrm{~A}$.

The rms value of control winding line current does not exceed over $10 \mathrm{~A}$ throughout the whole process, as shown in Figure 14, which entitles us to say that it is possible to further adjust the capacitor in order to get the capacity optimal through minimization of SEC for DSWIG wind power system with rectifier load.

\subsection{Experimental results}

The optimal selection algorithm and simulation were performed previously, followed by the experimental work. The experiments were carried out using an $18 \mathrm{~kW}$ six-phase/three-phase DSWIG wind power system prototype. The control software is soft coded in a Freescale MC56F8346 DSP-based development system. A three-leg Mitsubishi intelligent power module (IPM) is used for the buildup of the static excitation converter. The wind turbine dynamics will be emulated by a $20 \mathrm{~kW}$ three-phase induction motor driven by a Siemens M440 inverter. The excitation capacitor value is selected at $60 \mu \mathrm{F}$.

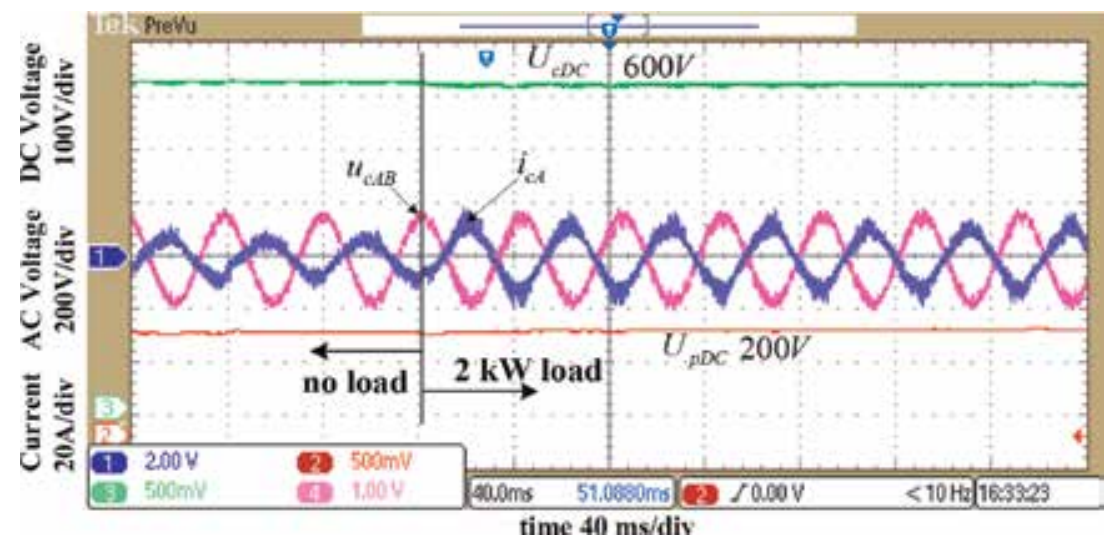

Figure 15.

Experimental waveform at $450 \mathrm{rev} / \mathrm{min}$ with $2 \mathrm{~kW}$ when the DSWIG outputs power from the control winding at low speed.

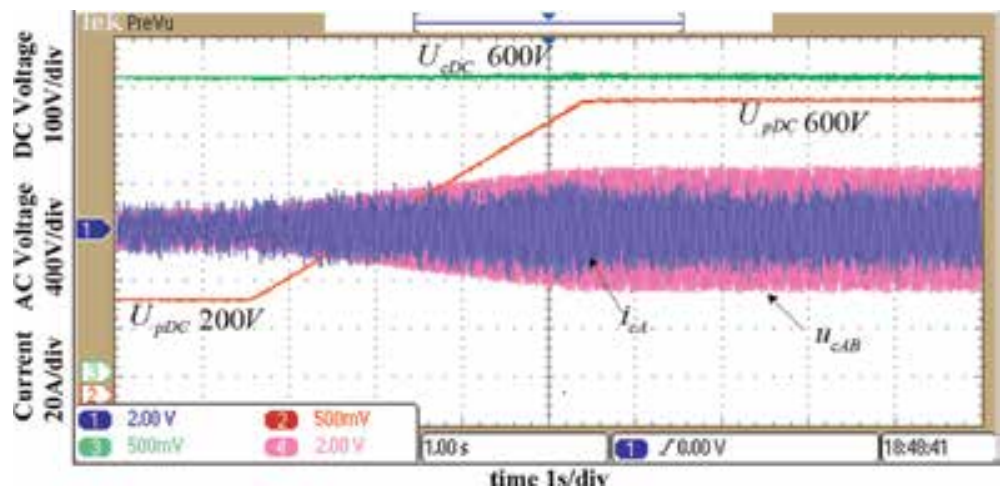

Figure 16.

Experimental waveform of the transition at $500 \mathrm{rev} / \mathrm{min}$. 
When the turbine shaft rotates at a minimum speed of $300 \mathrm{rev} / \mathrm{min}$, the system begins to build up voltage. The experimental results for this stage with the control strategy described above have been shown in Figure 14. It can be noticed that $U_{p D C}$ and $U_{c D C}$ rises with constant pace up to 200 and $600 \mathrm{~V}$, respectively, without any overshoot in voltage and current.

According to the theoretical analysis presented earlier above, when the speed is in the range of low wind speed, the rotor speed is low and the output power is small. In this case, the generated power is transferred through the SEC from the control

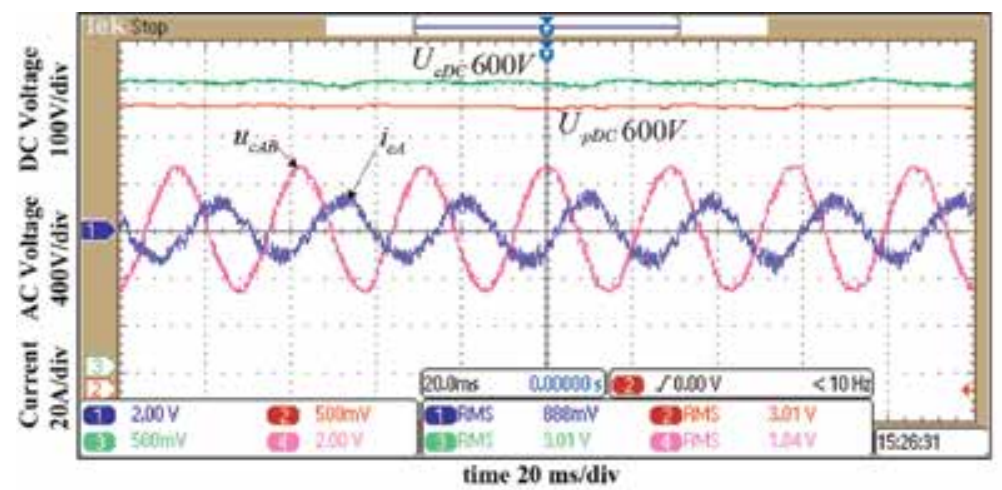

(a)

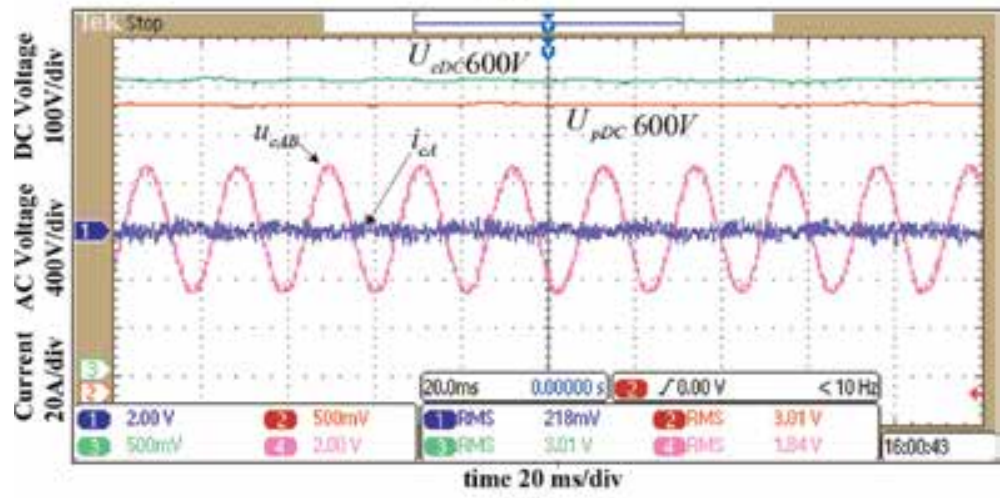

(b)

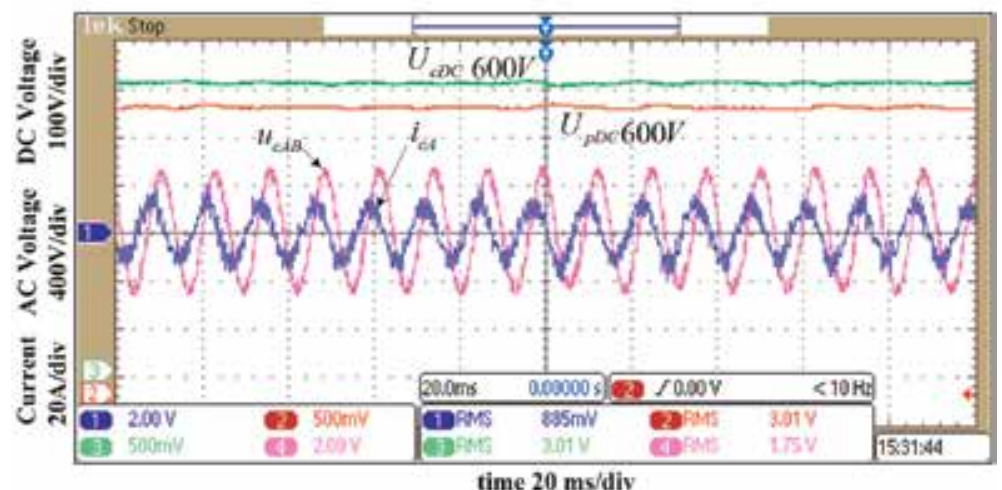

(c)

Figure 17.

DC bus voltage waveforms of the control winding line-to-line voltage and line current of DSWIG wind power system when the speed is increasing from 500 to $1200 \mathrm{rev} / \mathrm{min} \mathrm{b}$. (a) Light load, $3.5 \mathrm{~kW} 500 \mathrm{rev} / \mathrm{min}$, (b) $11 \mathrm{~kW}, 710 \mathrm{rev} / \mathrm{min}$, (c) rated load, $18 \mathrm{~kW}, 1200 \mathrm{rev} / \mathrm{min}$. 
winding. Figure 15 presents the experimental tests showing the characteristic waveform of the DSWIG wind power system spinning at $450 \mathrm{rev} / \mathrm{min}$ with $2 \mathrm{~kW}$ electric load. In this case, the rms value of control winding line current is about $8.5 \mathrm{~A}$.

The power winding voltage $U_{p D C}$ is controlled from 200 to $600 \mathrm{~V}$ when the speed reaches $500 \mathrm{rev} / \mathrm{min}$. The speed modification is followed by a change of the line-to-line voltage and line current of control winding. Through this transient state, $U_{c D C}$ is preserved at $600 \mathrm{~V}$ constant. The experimental test results show the transient state waveform of the voltage shown in Figure 16.

Figure 17 depicts the experimental results indicating the control winding lineto-line voltage and line current following several typical operating points/states of the energization process ranging from 500 to $1200 \mathrm{rev} / \mathrm{min}$. In Figure 17a, one may notice that the line current is phase shifted behind the line-to-line voltage no $<120^{\circ}$ (it is phase shifted behind the phase voltage almost $90^{\circ}$ electrical degrees), which indicates that the SEC will provide the reactive excitation power to the generator and the rms value is $8.9 \mathrm{~A}$. In a similar way, while the speed increases, the load of the generator accelerates. When the speed grows to $710 \mathrm{rev} / \mathrm{min}$, the output power of the generator is close to $11 \mathrm{~kW}$.

The reactive current provided by SEC in this situation is irrelevant, close to zero as it can be seen from Figure 17b. This can be explained by the fact that enough reactive excitation power required by the generator is provided by the excitation capacitor. The reactive excitation power provided by excitation capacitor decreases below the generator demand, while the speed and load are slowly increasing. This is the situation when the SEC must extract the supplementary reactive excitation power from the generator output. Over $800 \mathrm{rev} / \mathrm{min}$, the generation system will be capable to provide the rated power. The control winding line current and the corresponding line-to-line voltage waveforms when the speed is $1200 \mathrm{rev} / \mathrm{min}$ with $18 \mathrm{~kW}$ load are illustrated in Figure 17c. In this figure, the line current is phase shifted from the line-to-line voltage by $60^{\circ}$ (it is ahead of the phase voltage for about $90^{\circ}$ ). The control winding current of is about $8.8 \mathrm{~A}$ (the rms value).

The experimental results in Figure 18a presents the direct relationship between the rotor speed and output power of the DSWIG wind power system.
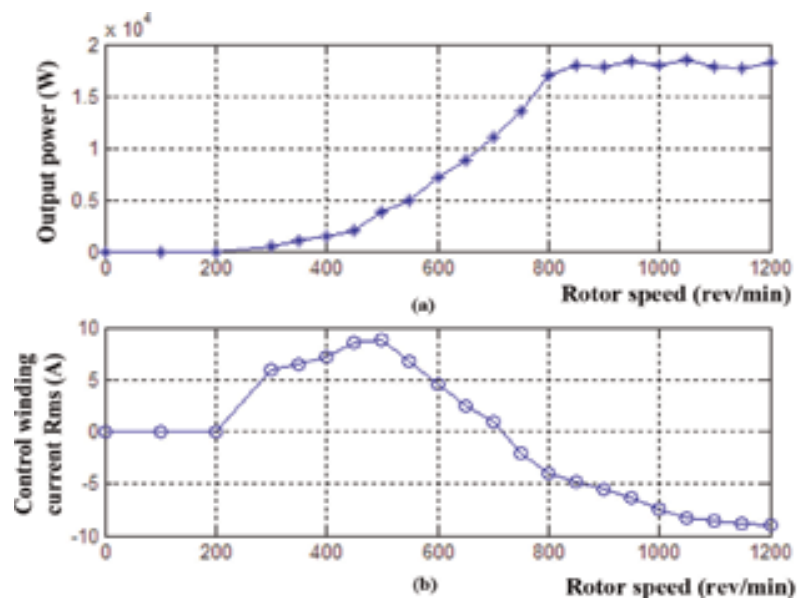

Figure 18.

Experimental results for output power and control winding line current of DSWIG wind power system from 300 to $1200 \mathrm{rev} / \mathrm{min}$. (a) Output power versus rotor speed. (b) Control winding line current versus rotor speed. 
Figure $18 b$, in a similar fashion, shows the modification of the control winding line current rms value during the generator transient regime. It can be noticed that the rms value of minimal negative current approximately equals to the maximum positive value, which is in correspondence with the theoretical analysis and simulation results. Furthermore, it is obvious that the maximum capacity of SEC is about $1 / 3$ of the rated output power. This is demonstrated by the experimental results, and the objective for a compact implementation of SEC is attained, which can lead to a reduction in the cost and size for the DSWIG wind power system.

\section{Conclusions}

In this chapter, novel dual stator-winding induction wind power generator system and its the optimal sizing algorithm of the excitation capacitor are presented. The work done here indicates that selection of optimal excitation capacitor value is highly dependent on generator parameters, speed range, and load but also is affected by the wind turbine power curve. Several methods are used to achieve the optimal excitation capacitor selection in order to reach the objective of minimizing the capacity of SEC.

We were using a complete model in the stator-voltage-orientation synchronous $\mathrm{d}-\mathrm{q}$ frame. Through the analysis of the model of SEC, the coupling between the $\mathrm{d}$ and $q$ axes of the DSWIG is underlined. Accordingly, a decoupling control strategy using the SVM is implemented at the level of hardware control. On the bases of this observation, the simulations and experiments from the prototype are carried out for verification. The results demonstrate the accuracy and soundness of this optimization algorithm for the DSWIG wind power system.

In this chapter, an optimal scheme is studied mainly, and it concentrates on the condition when relative parameters of the generator are identified. Additionally, the optimal design for the whole system including the generator should also be acknowledged. However, the optimization scheme obtained in this paper is rather a first attempt to the problem of topology design of multiphase induction machinebased generators and system optimization of the DSWIG system applied in wind power system.

\section{Appendices and nomenclature}

The six-phase/three-phase DSWIG parameters (both the rotor and control windings are converted to the power winding):

$R_{p}=0.624 \Omega ; R_{c}^{\prime}=0.434 \Omega ; R_{r}^{\prime}=0.32 \Omega ; L_{l p}=3.26 \mathrm{mH} ; L_{l r}^{\prime}=2.02 \mathrm{mH} ;$

$L_{l c}^{\prime}=3.12 \mathrm{mH} ; L_{m}=92.4 \mathrm{mH} ; L_{l m p 12}=L_{l m c p 1}=L_{l m c p 2}=0.42 \mathrm{mH}$.

Rated power: $18 \mathrm{~kW}$.

Rated speed: $750 \mathrm{rev} / \mathrm{min}$.

Pole pairs: 4 .

Rated DC output voltage: $600 \mathrm{~V}$.

Filter inductor: $3 \times 4 \mathrm{mH}$.

Excitation capacitor: $6 \times 60 \mu \mathrm{F}$.

DC bus capacitor: $1100 \mu F / 900 \mathrm{~V}$.

Interphase reactor: $9 \mathrm{mH}$.

Voltage of battery: $48 \mathrm{~V}$. 


\section{Author details}

Sorin Ioan Deaconu ${ }^{1 *}$, Feifei Bu ${ }^{2}$, Marcel Topor ${ }^{1}$, Lucian Nicolae Tutelea ${ }^{1}$ and Nicolae Muntean ${ }^{1}$

1 Politehnica University Timisoara, Romania

2 University of Aeronautics and Astronautics, Nanjing, China

*Address all correspondence to: sorin.deaconu@fih.upt.ro

\section{IntechOpen}

(C) 2019 The Author(s). Licensee IntechOpen. This chapter is distributed under the terms of the Creative Commons Attribution License (http://creativecommons.org/licenses/ by/3.0), which permits unrestricted use, distribution, and reproduction in any medium, provided the original work is properly cited. (cc) BY 


\section{References}

[1] Global Wind Energy Council. Global Wind Statistics 2012. 2013

[2] Chen W, Huang AQ, Li C, Wang G, $\mathrm{Gu}$ W. Analysis and comparison of medium voltage high power DC/DC converters for offshore wind energy systems. IEEE Transactions on Power Electronics. 2013;28(4):2014-2023

[3] Prasai A, Yim J, Divan D, Bendre A, Sul S. A new architecture for offshore wind farms. IEEE Transactions on Power Electronics. 2008;23(3): 1198-1204

[4] Kirby NM, Xu L, Luckett M, Siepmann W. HVDC transmission for large offshore wind farms. Power Engineering Journal. 2002;16(3):135-141

[5] Li H, Chen Z. Overview of different wind generator systems and their comparisons. IET Renewable Power Generation. 2008;2(2):123-138

[6] Echenique E, Dixon J, Cardenas R, Pena R. Sensorless control for a switched reluctance wind generator based on current slopes and neural networks. IEEE Transactions on Industrial Electronics. 2009;56(3): 817-825

[7] Leijon M, Dahlgren M, Walfridsson L, Li M, Jaksts A. A recent development in the electrical insulation systems of generators and transformers. IEEE Electrical Insulation Magazine. 2001;17(3):10-15

[8] Egea-Alvarez A, Bianchi F, JunyentFerre A, Gross G, Gomis-Bellmunt O. Voltage control of multiterminal VSCHVDC transmission systems for offshore wind power plants: Design and implementation in a scaled platform. IEEE Transactions on Industrial Electronics. 2013;60(6):2381-2391

[9] Muyeen SM, Takahashi R, Tamura J. Operation and control of HVDC- connected offshore wind farm. IEEE Transactions on Sustainable Energy. 2010;1(1):30-37

[10] Bozhko SV, Blasco-Gimenez R, Li R, Clare JC, Asher GM. Control of offshore DFIG-based wind farm grid with linecommutated HVDC connection. IEEE Transactions on Energy Conversion. 2007;22(1):71-78

[11] Bansal RC, Bhatti TS, Kothari DP. Bibliography on the application of induction generators in nonconventional energy systems. IEEE Transactions on Energy Conversion. 2003;18(3):433-439

[12] Hu Y, Huang W, Li Y. A novel instantaneous torque control scheme for induction generator systems. IEEE Transactions on Energy Conversion. 2010;25(3):795-803

[13] Zhang J, Rahman MF. A directflux-vector-controlled induction generator with space-vector modulation for integrated starter alternator. IEEE Transactions on Industrial Electronics. 2007;54(5):2512-2520

[14] Leidhold R, Garcia G, Valla MI. Induction generator controller based on the instantaneous reactive power theory. IEEE Transactions on Energy Conversion. 2002;17(3):368-373

[15] Ojo O, Davidson IE. PWM-VSI inverter-assisted stand-alone dual stator winding induction generator. IEEE Transactions on Industry Applications. 2000;36(6):1604-1611

[16] Wang D, Ma W, Xiao F, Zhang B, Liu D, Hu A. A novel stand-alone dual stator-winding induction generator with static excitation regulation. IEEE Transactions on Energy Conversion. 2005;20(4):826-835

[17] Li Y, Hu Y, Huang W, Liu L, Zhang Y. The capacity optimization for 
the static excitation controller of the dual-stator-winding induction generator operating in a wide speed range. IEEE Transactions on Industrial Electronics. 2009;56(2):530-541

[18] Bu F, Huang W, Hu Y, Shi K. An excitation-capacitor-optimized dual stator-winding induction generator with the static excitation controller for wind power application. IEEE Transactions on Energy Conversion. 2011;26(1): 122-131

[19] Liu L, Hu Y, Huang W. Optimal design of dual stator-winding induction generator with variable speed based on improved genetic algorithm. In: 2005 The 8th International Conference on Electrical Machines and Systems, ICEMS. 2005. pp. 2343-2348

[20] Zhu W, Wang X, Wang X. Optimal wind power capturing operational mode of a variable-speed variable-frequency wind power generation system. Automation of Electric Power Systems (China). 2009;33(11):94-98

[21] Koutroulis E, Kalaitzakis K. Design of a maximum power tracking system for wind-energy-conversion applications. IEEE Transactions on Industrial Electronics. 2006;53(2): 486-494

[22] Li Y, Hu Y, Liu L, Huang W, Chen G. Control of a novel dual statorwinding induction generator for wide speed-range operation. In: IEEE Power Electronics Specialists Conference. 2007. pp. 2096-2101

[23] Hu Y, Huang W, Zhang L. Research on employing starter/generator system. Transactions of China Electrotechnical Society. 2006;2(5):7-13

[24] Peng F, Lai J. Generalized instantaneous reactive power theory for three-phase power systems. IEEE Transactions on Instrumentation and Measurement. 1996;45(1):293-297
[25] Li Y, Hu Y, Huang W, Qiu J, Chen G, Hao Z. Voltage control of dual stator-winding induction generator system using space vector modulation. Proceedings of Chinese Society Electrical Engineering. Aug 2008;28 (23):112-118 


\title{
A Simple Hybrid Electric Vehicle Fuel Consumption Model for Transportation Applications
}

\author{
Kyoungho Ahn and Hesham A. Rakha
}

\begin{abstract}
This study presents a simple power-based microscopic hybrid electric vehicle (HEV) fuel consumption model for use in microscopic traffic software and various connected and automated vehicle (CAV) applications, including eco-routing and eco-drive systems. While numerous HEV energy consumption models have been developed, these models are complex and require vehicle engine data deeming them difficult to implement and making them nontransferable. The proposed model was developed using empirical data for a 2010 Toyota Prius-the most popular HEV. The model was then extended to other HEVs thus extending the domain of application of the model. The proposed fuel consumption model estimates the instantaneous fuel consumption rates of an HEV using instantaneous vehicle operational input variables, including the vehicle's speed, acceleration, and roadway grade, which can be acquired from global positioning system (GPS) equipment or other sensors. The model estimates vehicle fuel consumption rates consistent with empirical data producing an average error of $2.1 \%$ for the Toyota Prius and up to $4 \%$ for other HEVs demonstrating the applicability and transferability of the model to various HEVs.
\end{abstract}

Keywords: HEV, energy model, fuel consumption model, Toyota Prius, microscopic model

\section{Introduction}

This study develops a microscopic hybrid electric vehicle (HEV) fuel consumption model that can be implemented in various transportation applications. In 2017, the transportation sector accounted for $\sim 71 \%$ of the world's total primary energy consumption [1]. Carbon dioxide $\left(\mathrm{CO}_{2}\right)$ emissions account for $98 \%$ of US emissions. Pollutions are mainly produced from the traditional combustion engines, including gasoline, diesel, and other heavy oils. Among fossil fuels, petroleum accounts for $92 \%$ of the total transportation energy consumption in the United States [2, 3].

Electric vehicles (EVs) utilize electricity for vehicle powertrain as either primary or to assist conventional vehicle designs. EVs are categorized as battery-only electric vehicles (BEVs), HEVs, and plug-in hybrid electric vehicles (PHEVs). HEVs use both a traditional engine and an electric motor. However, while PHEVs can plug into an electric source to charge a battery, HEVs are charged through regenerative braking and by the internal combustion engine and cannot be plugged in to charge the battery [4]. HEVs are more fuel efficient and produce lower tailpipe emissions 
than the conventional internal combustion engine vehicles (ICEVs) and are typically easier and cheaper to build than PHEVs.

Connected vehicles $(\mathrm{CVs})$ are an emerging technology that connects vehicles to other vehicles and to the road infrastructure using various vehicle communication technologies. CV technology is expected to generate transformative improvements in the roadway transportation system and will extend the benefits of intelligent transportation systems (ITSs) to entire networks by improving the efficiency, safety, energy consumption, and environmental footprint of the transportation system through the real-time exchange of information among vehicles and infrastructure. These technologies can assist drivers in avoiding congestion, reducing vehicle stops, and achieving optimal fuel efficiency. In order to evaluate the energy saving and the fuel efficiency of CV applications, simple and efficiency energy model is required. Many CV applications focus on improving the energy efficiency of various vehicle types, including electric vehicles (EVs). However, few fuel consumption models for HEVs have been developed for these CV applications.

A number of fuel/energy consumption models have been developed and employed to estimate the energy and environmental impacts of various transportation projects. Most models were developed with a special interest in measuring fuel consumption for transportation planning, transportation impact assessments, vehicle technology evaluations, traffic simulation models, and specific control conditions. Some models were developed to estimate the total fuel consumed and emissions of an entire network, city, or state, while other models were intended for a corridor or an intersection. While various models perform effectively their specific purposes, there are several requirements for an ideal fuel consumption and emission model that can accommodate real-time CV applications. A previous study [5] recommended four major criteria for eco-driving fuel consumption models: realtime computation, accuracy, model structure, and model calibration simplicity. Currently there are a number of HEV energy models; few HEV energy consumption models are eligible for real-time CV applications.

The objective of this study is to develop a microscopic HEV fuel consumption model that can be incorporated in CV applications. The proposed model estimates the instantaneous fuel consumption of HEVs using instantaneous vehicle operational input variables, including the vehicle's speed, acceleration, and roadway grade.

The paper is organized as follows: the next section reports the state-of-the art of HEV energy consumption efforts. Then, the paper describes the HEV fuel consumption data that were utilized for this study. The following section describes the proposed model development and validation. Finally, the conclusions of the study are presented.

\section{Literature review}

There are three types of HEV powertrain systems including series, parallel, and series/parallel systems [6]. A series powertrain system, which is typically efficient at stop and go operational conditions, uses electric power from either the battery or a generator produced by a vehicle engine. The series HEV system uses the gas engine to produce electricity for the electric motor. The series HEV controls the required power from the battery or the generator. Parallel HEVs utilize the battery electric motor and the engine in tandem. These vehicles typically use a small battery compared to series HEVs and operate the motor as a generator for recharging particularly when extra power is required [7]. Also these parallel HEVs are more efficient for highway driving conditions because the engine is directly connected to 
the wheels. Series/parallel powertrain HEVs combine the benefits of other two HEV types [8-10]. Xiong et al. proposed a minimum-fuel energy control strategy by developing a fuel-optimum power management strategy of series/parallel hybrid [9]. Staunton et al. introduced the framework for a hybrid power control system and evaluated the Toyota Prius hybrid powertrain system [8]. The Toyota Prius is one of the most popular series/parallel drivetrain designs. The vehicle operates as a series HEV at low-speed and as a parallel HEV at high-speed conditions.

For all types of powertrains, the energy management strategies of most commercialized HEVs typically adopt rule-based methods to simplify switching operating modes $[10,11]$. Mansour and Clodic demonstrated the model and design of the Toyota Prius hybrid powertrain system and developed a dynamic model of the electromechanical configuration [10]. Lin et al. designed a near-optimal power management strategy for a parallel hybrid vehicle and developed a fuel-optimum power management strategy [11].

A number of studies have developed control algorithms for HEVs. Barsali et al. developed a power control strategy to minimize fuel consumption for a series hybrid vehicle [6], and Delprat et al. developed an optimal control strategy for parallel hybrid vehicles by building a computationally efficient power control strategy to minimize fuel consumption [7]. Gao et al. proposed a fuel-optimized power control strategy for a series hybrid electric bus and developed a minimum-fuel power control strategy for a series hybrid electric vehicle [12].

Rule-based strategies are basically designed according to engineering intuition and simple analysis of component efficiency tables/charts [13]. For instance, Bowles developed a powertrain model and energy control strategy for a parallel hybrid vehicle and described new energy management strategies of HEVs.

A number of recent studies have investigated the impacts of the fuel/energy consumption of HEVs. For example, Fontaras investigated HEV fuel economy and pollutant emissions by measuring fuel consumption and emissions using various drive cycles. Fontaras et al. found that HEVs could save energy consumption and reduce vehicle emissions by up to $60 \%$ under urban driving conditions compared to ICEVs [14]. Al-Samari investigated the energy efficiency benefits of using HEVs in comparison to conventional vehicles using Autonomie and found that the fuel economy could be improved significantly (up to 68\%) in a real-world driving cycle consisting of mostly city activity and up to $10 \%$ highway driving [15]. Oak Ridge National Laboratory researchers quantified the effects of aggressive driving with HEVs and the limitation of regenerative braking for HEVs. The study concluded that the limitation is a major reason to a bigger variation of HEV fuel efficiency data than the ICEV data [16].

The advanced vehicle simulator (ADVISOR) developed by the National Renewable Energy Laboratory (NREL) is one of the most popular models for estimating the fuel consumption of HEVs [17]. However, due to its complex modeling structure, it would be very difficult to implement ADVISOR into real-time eco-driving applications and CV applications. On the other hand, Boubaker et al. introduced an HEV energy model that employs a simplified module with two motors/generators (MGs) and planetary gear train (PGT) that estimates fuel consumption based on the engine speed (or RPM) and the output torque of the engine. However, the model also requires MATLAB/Simulink software to estimate model variables due to the complexity and was not validated against real-world data [18].

In recent years, a number of studies have investigated the modeling of $\mathrm{HEV}$ energy consumption. However, most of the developed HEV models are not general, cannot be calibrated to specific vehicles, or cannot be used in CV applications, due to the complicated vehicle-specific data requirements and/or the modeling structure. This study overcomes these problems by developing a new HEV energy model 
that is general and transferable and can be easily used in various transportation applications. The proposed model does not require engine efficiency maps and calculates the fuel consumption of HEVs based on driving dynamics using vehicle speed, acceleration, and roadway grade data.

\section{HEV energy consumption data}

The study utilized a test dataset that was collected at the Advanced Powertrain Research Facility (APRF) at the Argonne National Laboratory. The test vehicle, a 2010 Toyota Prius, includes an Atkinson-cycle engine, two electric motors, and a power-split device used to control the allocation of energy between the electric and mechanical (fuel) power paths [19]. The data were downloaded from the Downloadable Dynamometer Database. The database is managed by the US Department of Energy (US DOE). APRF was built for vehicle benchmarking for the US DOE and utilizes two-wheel chassis dynamometers and four-wheel drive chassis

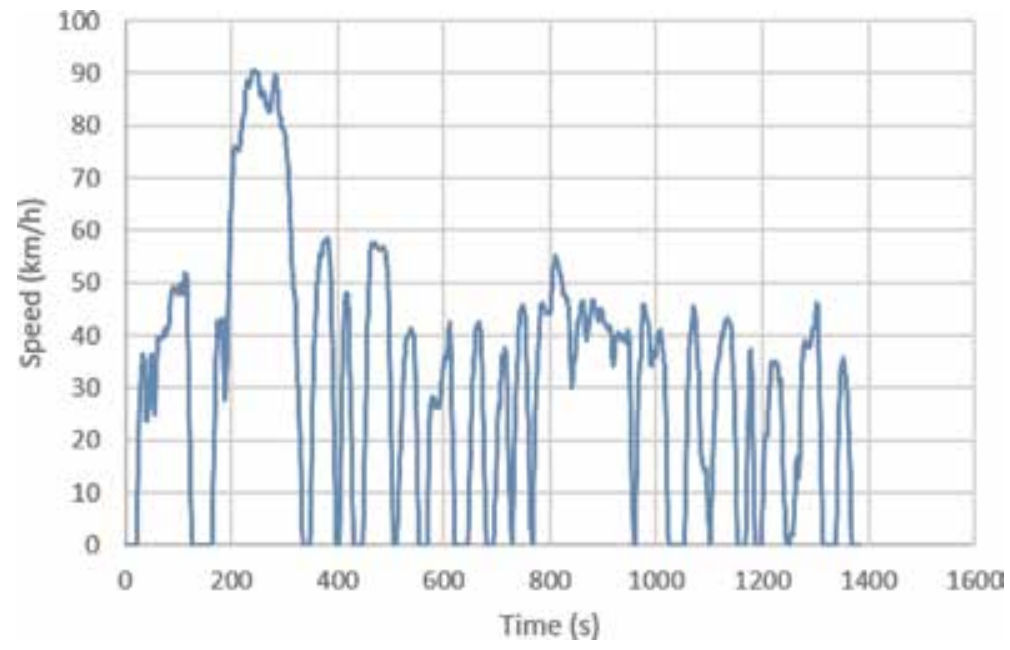

Figure 1.

UDDS (city) driving cycle.

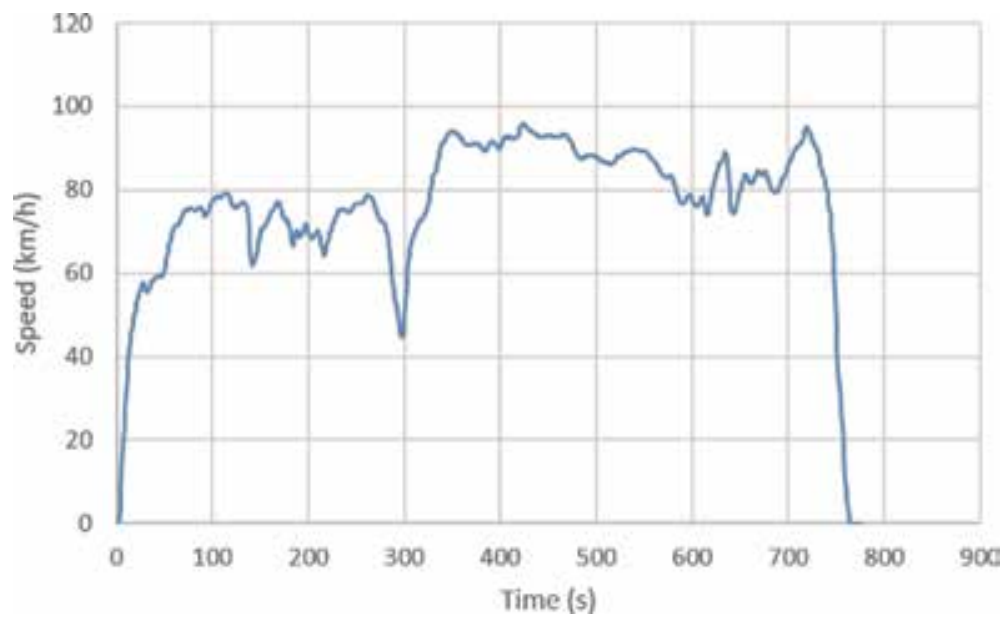

Figure 2.

Highway driving cycle. 
dynamometers to collect vehicle energy consumption data, including both fuel and electricity, emissions, powertrain performance, and vehicle operational data. The test Toyota Prius data were collected using a two wheels chassis dynamometer.

APRF collected fuel consumption data for driving cycles: the Urban Dynamometer Driving Schedule (UDDS) (also referred to as the city test or LA4 cycle), Highway Fuel Economy Test (HWFET), the US06 cycle, and the steady-state speed cycle (or step speed cycle). This study utilized only hot-start trip data to only consider hot-stabilized conditions. Figures 1-4 show the speed profiles of the four driving cycles. The UDDS represents city driving conditions and is typically used for light duty vehicle testing. The UDDS utilizes the same driving cycles of the Federal Test Procedure (FTP) without a cold start section, which is the first 505 seconds. The HWFET represents highway driving conditions at $96 \mathrm{~km} / \mathrm{h}$ (60 mph). The US06 cycle is considered a "supplemental FTP" driving schedule and represents high-speed and aggressive-acceleration driving conditions. The steady-state speed cycle represents various steady-state speeds up to $128 \mathrm{~km} / \mathrm{h}$ (80 $\mathrm{mph}$ ).

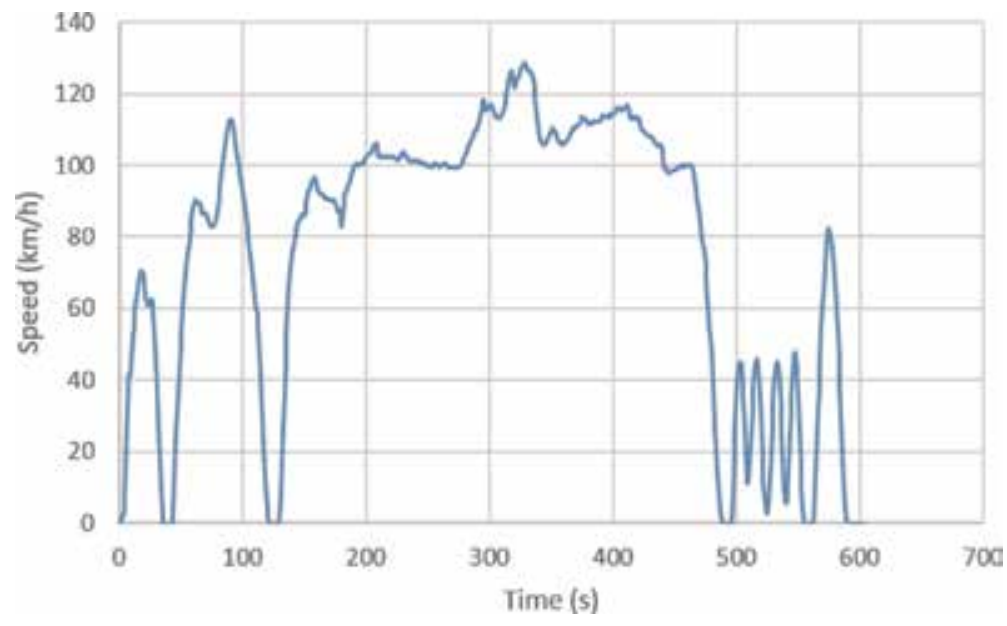

Figure 3.

USo6 driving cycle.

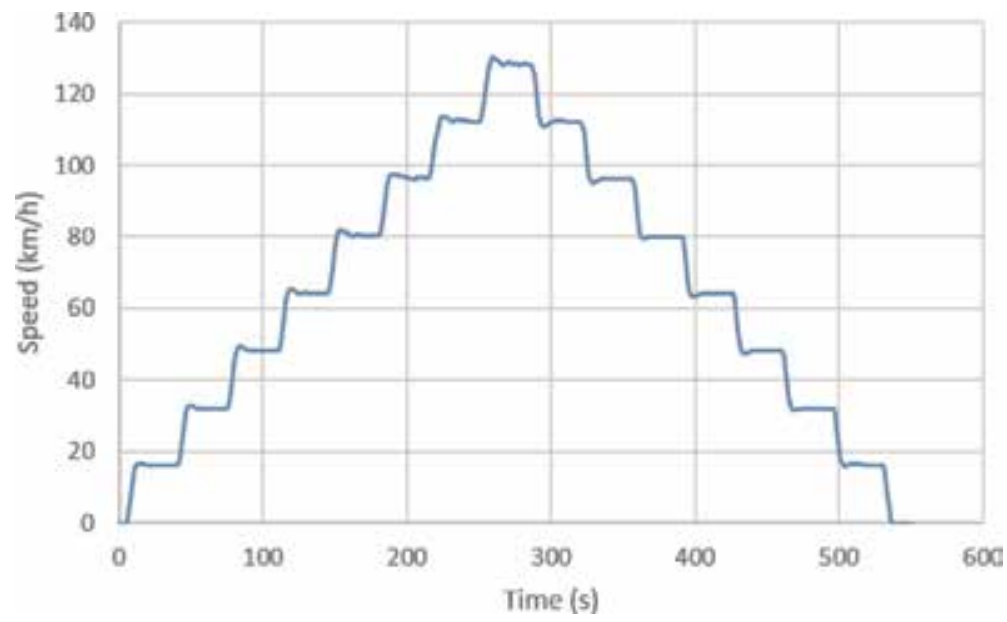

Figure 4 .

Steady-state speed driving cycle. 


\section{HEV energy consumption modeling}

The study utilized a test dataset that was collected at the Advanced Powertrain Research Facility at the Argonne National Laboratory. The test vehicle, a 2010 Toyota Prius, includes an Atkinson-cycle engine, two electric motors, and a powersplit device used to control the allocation of energy between the electric motor and the vehicle engine.

It is difficult to estimate HEV fuel consumption due to the complexity of the various ICE engine and electrical components. The powertrain of an HEV includes an internal combustion engine (ICE), two electric motors/generators (MG1 and MG2), a PGT, and a battery [18].

Figures 5 and 6 illustrate the variations in the vehicle fuel consumption for the UDDS cycle and the steady-state speed cycle. As illustrated in the figures, the fuel consumption rates are significantly affected by vehicle speed and acceleration levels. The figure demonstrates that the HEV uses only electric mode when significant vehicle power is not required, as shown in the case of the steady-state speed cycle.

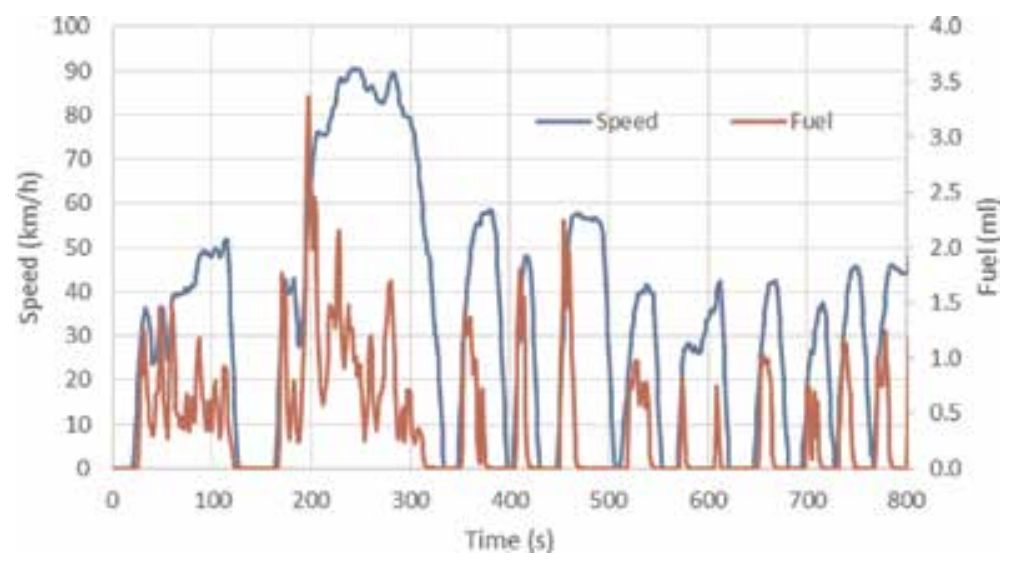

Figure 5.

Fuel consumption of UDDS cycle.

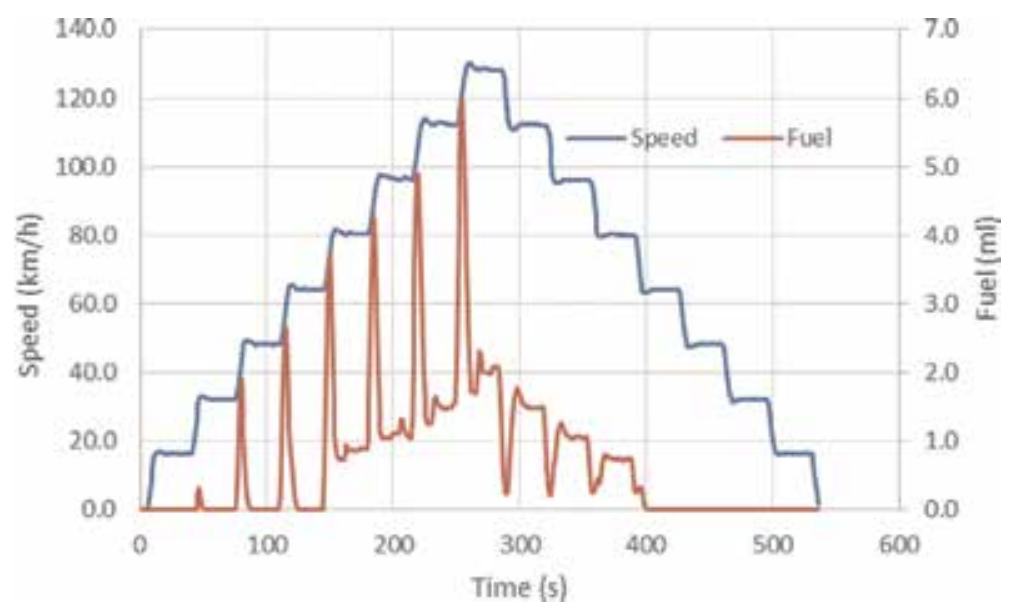

Figure 6.

Fuel consumption of steady-state speed cycle. 
Figure 7 illustrates the relationship between vehicle speed and the fuel consumption rate from the Toyota Prius data. Figure 8 shows the relationship between vehicle speed and power. The figures clearly show that fuel consumption is closely related to vehicle speed and power; as the test vehicle's speed increased, vehicle power increased proportionally.

We compared the speed observation and fuel data and found that there were time lags. In order to remove the temporal offsets, we utilized a procedure for offsetting the data and operational variables that was developed earlier [20] and applied it to the 2010 Toyota Prius data. We analyzed the test data and found the following HEV energy consumption behaviors. First, the amount of fuel consumed is proportionally related to both the vehicle power and speed; second, the HEV operates in EV mode when the power is negative; and third, the HEV utilizes only an electric mode when the speed is lower than an EV mode speed $\left(v_{a}\right)$ and the

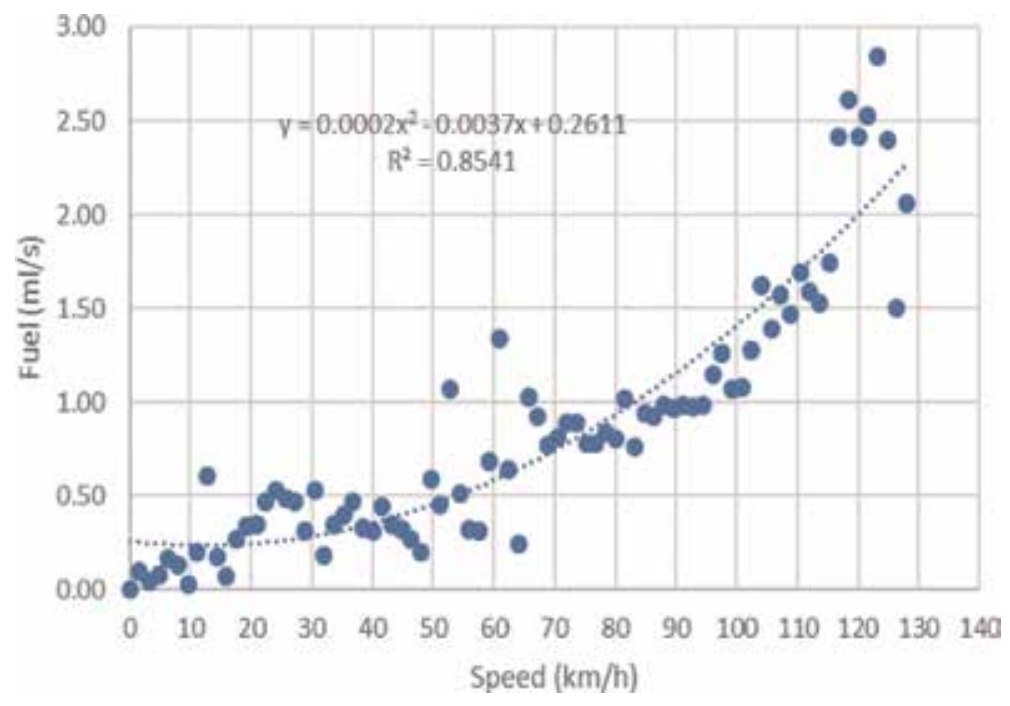

Figure 7.

Relationship between vehicle speed and fuel consumption.

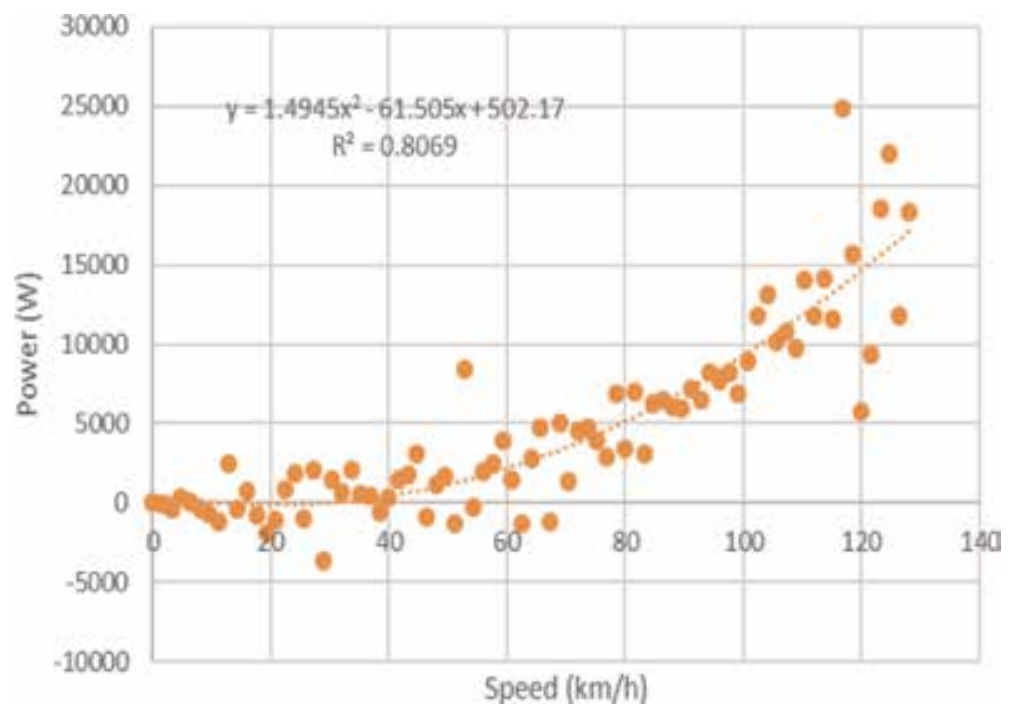

Figure 8.

Relationship between vehicle speed and power. 
required power is lower than a specific power $\left(P_{a}\right)$. Consequently, the proposed fuel consumption model is formulated in Eq. (1):

$$
F C(t)=\left\{\begin{array}{cc}
F C_{E V \_ \text {mode }} & \text { for }\left\{\begin{array}{c}
P \leq 0 \\
v<v_{a} \text { and } P<P_{a}
\end{array}\right. \\
a+b * v(t)+c * P(t)+d * P(t)^{2} & \text { for }\left\{\begin{array}{c}
P>0 \text { and } v \geq v_{a} \\
v<v_{a} \text { and } P \geq P_{a}
\end{array}\right.
\end{array}\right.
$$

where $F C(t)$ is the fuel consumption rate $(\mathrm{ml} / \mathrm{s})$ and $F C_{E V_{-} \text {mode }}$ is the fuel consumption rate $(\mathrm{ml} / \mathrm{s})$ in $\mathrm{EV}$ mode and estimated as average fuel consumption in EV mode; $P(t)$ is the instantaneous total power in kilowatts $(\mathrm{kW})$; and $v$ is the instantaneous vehicle speed in kilometers per hour. Statistical analysis of the empirical data found that the optimum values for $v_{a}$ and $P_{a}$ are $32 \mathrm{~km} / \mathrm{h}$ and $10 \mathrm{~kW}$, respectively. The model coefficients $a, b, c$, and $d$ for the 2010 Toyota Prius are $0.006,0.003998,0.077092$, and $-9.155 \mathrm{E}-05$, respectively.

\section{Model validation}

Table 1 provides the fuel consumption results of the proposed model. The total fuel consumption for the UDDS, Highway, US06, and steady-state speed cycles, as measured in the laboratory, was $403.4,576.8,705.9$, and $388.9 \mathrm{ml}$, respectively. The

\begin{tabular}{lccc}
\hline Driving cycles & Raw data $(\mathbf{m l})$ & Estimated fuel (ml) & Trip error (\%) \\
\hline UDDS & 403.4 & 387.1 & -4.1 \\
\hline Highway & 576.8 & 611.2 & +6.0 \\
\hline US06 & 705.9 & 668.2 & -5.3 \\
\hline Steady-state speed & 388.9 & 365.7 & -6.0 \\
\hline Total & 2075.0 & 2032.1 & -2.1 \\
\hline
\end{tabular}

Table 1.

Fuel consumption results.

\section{UDDS Cycle}

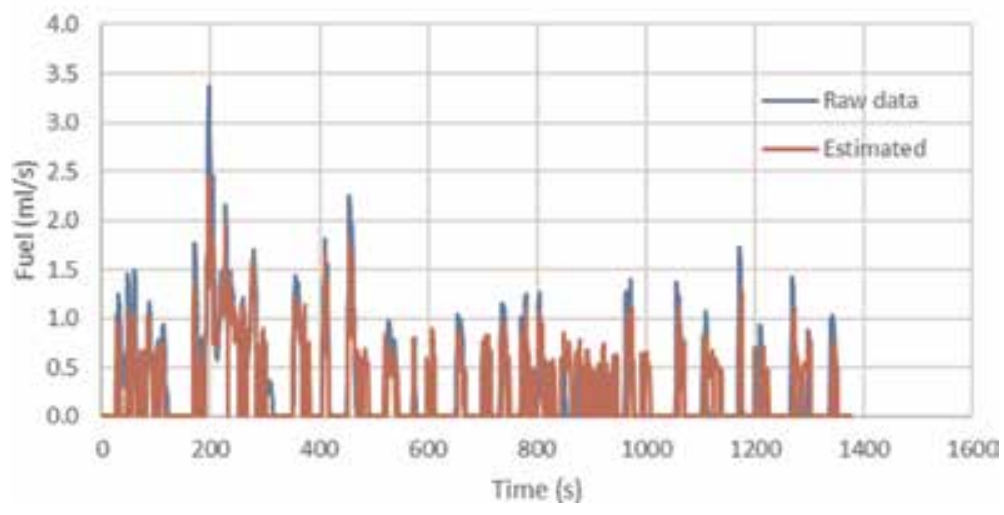

Figure 9.

Instantaneous model validation (UDDS cycle). 

DOI: http://dx.doi.org/10.5772/intechopen.89055

estimated fuel consumptions based on the proposed model were 387.1, 611.2, 668.2, and $365.7 \mathrm{ml}$, corresponding to a 4.1, 6.0, 5.3, and 6.0\% difference in overall fuel consumption for each driving cycle.

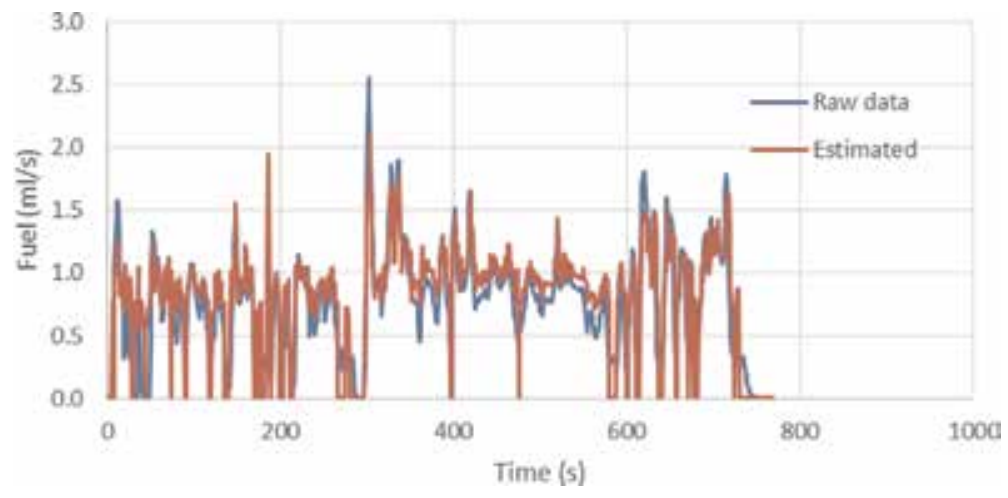

Figure 10.

Instantaneous model validation (highway cycle).

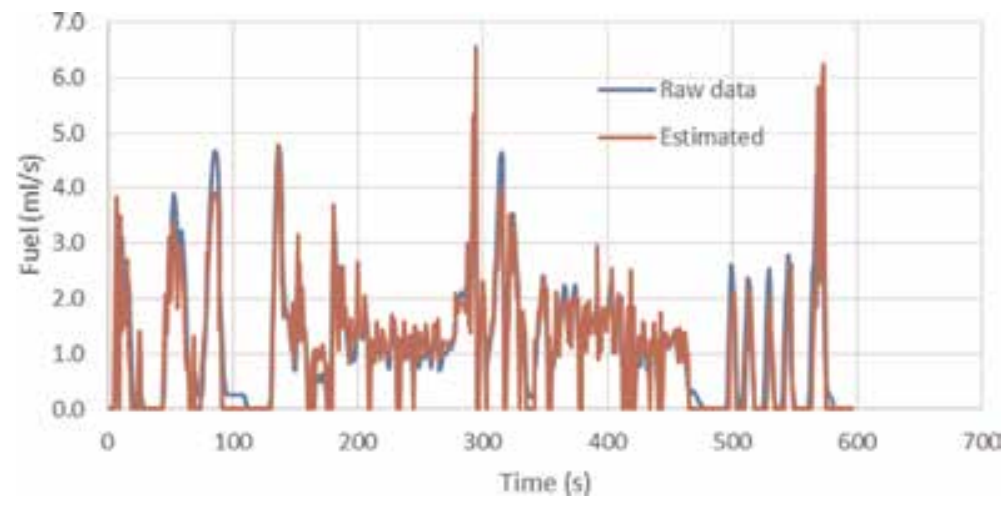

Figure 11.

Instantaneous model validation (USo6 cycle).

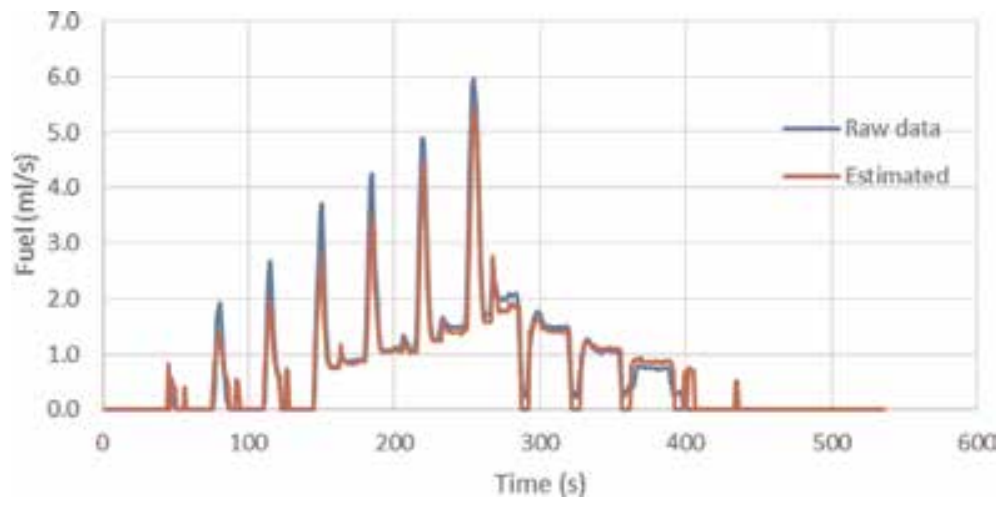

Figure 12.

Instantaneous model validation (steady-state speed cycle). 
Figures 9-12 illustrate the test vehicle's measured instantaneous fuel consumption rate for the four different driving cycles. The model estimates were calculated based on instantaneous power and vehicle speed levels. The figures show that the estimated fuel consumption generally follows the raw fuel consumption data. However, we found that the proposed model slightly overestimates or underestimates some fuel consumption rates for the four driving cycles. In particular, the proposed model slightly underestimates fuel consumption rates when the test vehicle accelerates from a steady-state speed. While the proposed model simplifies the conditional EV mode, the EV mode also depends on the state of charge (SOC) of the battery. The team will further investigate the impacts of SOC to improve the accuracy of the fuel consumption model. In general, however the model predictions follow the laboratory-collected fuel measurements of the steady-state speed cycle with a high correlation coefficient (0.951). The accuracy of the proposed model is good enough to assess the effects of transportation projects, including eco-driving and CV applications, to improve vehicle fuel economy.

\section{HEV scale factor development}

As demonstrated earlier, the 2010 Toyota Prius HEV energy model provides sufficient accuracy for use in various HEV applications. The study expanded the HEV fuel model to estimate the fuel consumption of various HEVs without specific calibration procedures. We tested various HEV fuel consumption models and found that the proposed scale factor method was the most accurate and efficient method. In particular, we utilize the EPA fuel economy data to estimate the second-bysecond fuel consumption of HEVs. For example, the fuel economy of the 2010 Toyota Prius is 51 and $49 \mathrm{mpg}$ for the city and highway cycles and $50 \mathrm{mpg}$ for the EPA combined fuel economy. The fuel economy of the 2013 Honda Civic Hybrid is 43 and $44 \mathrm{mpg}$ for the city and highway cycles, respectively, and $43.5 \mathrm{mpg}$ for the EPA combined fuel economy. For the Honda Civic Hybrid, the relative difference of the combined fuel economy is $1.14(50 / 43.5)$. We utilized this scale factor to

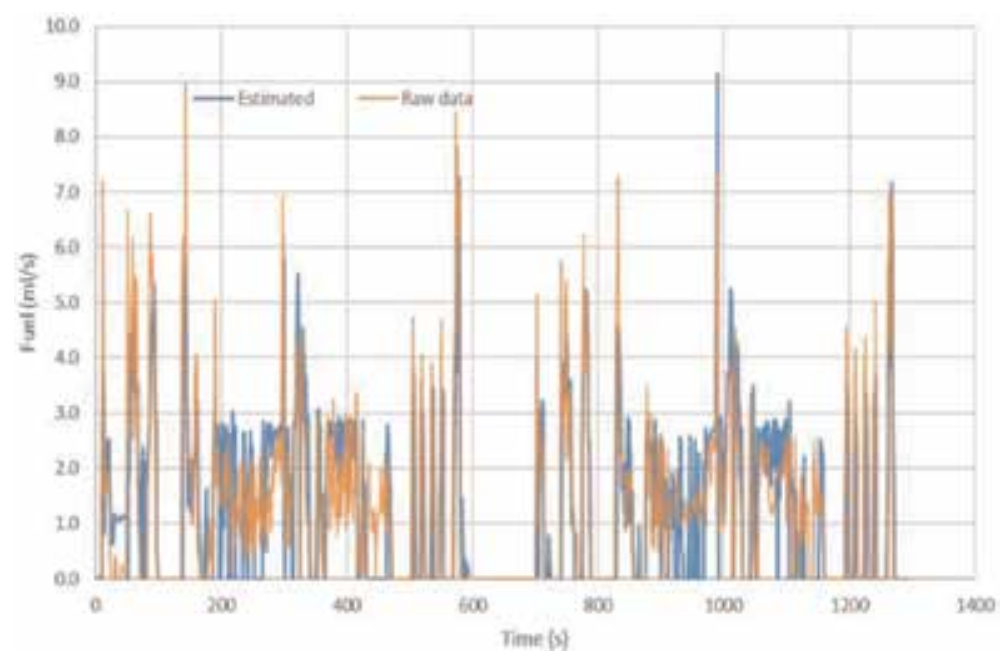

Figure 13.

Instantaneous model validation (2013 VW Jetta hybrid). 


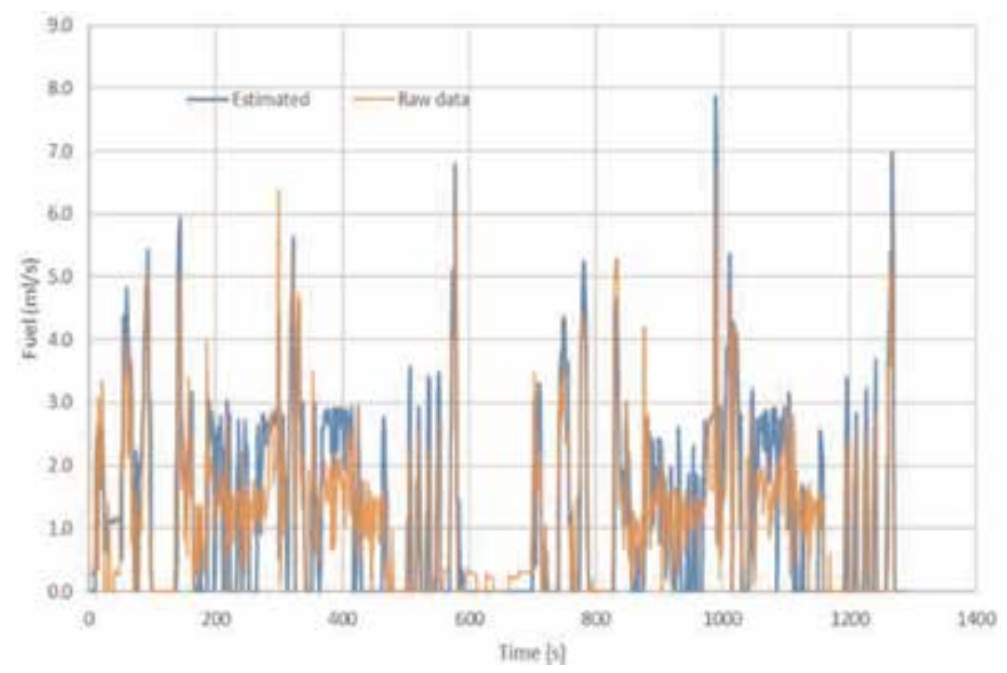

Figure 14.

Instantaneous model validation (2013 Honda civic hybrid).

estimate the energy consumption for the Honda Civic HEV. Consequently, the proposed fuel consumption model is formulated in Eq. (2):

$$
F C(t)=\left\{\begin{array}{cl}
\left(F C_{E V_{\text {mode }}}\right) \times S C_{i} \text { for } & \left\{\begin{array}{c}
P \leq 0 \\
v<v_{a} \text { and } P<P_{a}
\end{array}\right. \\
\left(a+b * v(t)+c * P(t)+d * P(t)^{2}\right) \times S C_{i} \text { for }\left\{\begin{array}{c}
P>0 \text { and } v \geq v_{a} \\
v<v_{a} \text { and } P \geq P_{a}
\end{array}\right.
\end{array}\right.
$$

where $S C_{i}$ is the scale factor of vehicle $i$ using the same $v_{a}$ and $P_{a}$ values of $32 \mathrm{~km} / \mathrm{h}$ and $10 \mathrm{~kW}$ for the all test vehicles. Figures 13-15 compare the measured and the estimated instantaneous fuel consumption rates for three different HEVs.

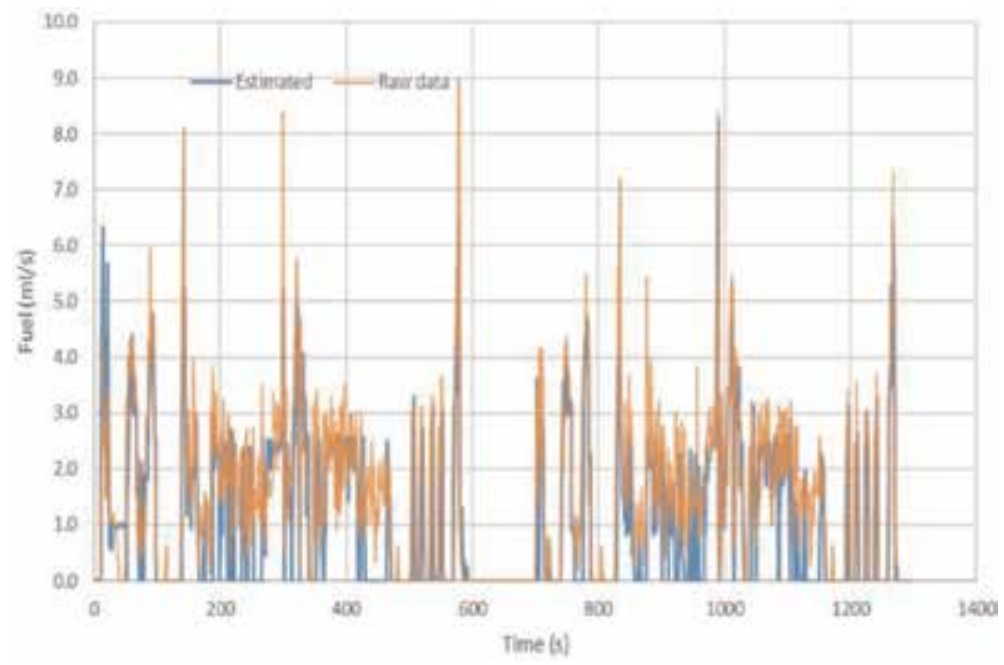

Figure 15.

Instantaneous model validation (2010 ford fusion hybrid). 
We tested four HEVs that include a 2013 VW Jetta Hybrid, a 2013 Honda Civic Hybrid, and a 2010 Ford Fusion Hybrid. For the evaluation, we utilized the US06 driving cycle since it is a high speed and acceleration cycle and has aggressive driving behaviors with an average speed of $48.4 \mathrm{mph}(77.9 \mathrm{~km} / \mathrm{h})$ and a maximum speed of $80.3 \mathrm{mph}(129.2 \mathrm{~km} / \mathrm{h})$. As illustrated in the figures, the predicted fuel consumption generally follows the second-by-second measured fuel consumption. The measured fuel consumption for the 2013 VW Jetta Hybrid, 2013 Honda Civic Hybrid, and 2010 Ford Fusion Hybrid were 1.625, 1.583, and 1.924 L and the estimated fuel consumption was $1.564,1.604$, and $1.845 \mathrm{~L}$, respectively. Consequently, the errors were 4,1 , and $4 \%$, demonstrating that the proposed model could be utilized for various HEVs.

\section{HEV state of charge evaluation}

The state of charge (SOC) of the battery affects the energy consumption of EVs. Since HEVs can use an internal combustion engine, the relative impacts of SOC for HEV are smaller than the other EVs. However, when the SOC is low, HEVs must utilize an engine instead of using EV mode, and it reduces the fuel economy of HEVs. Thus, the EV mode of HEVs depends on the SOC of the battery. This study investigated how the SOC of HEVs changes during a trip.

Figure 16 illustrates the measured and the estimated SOC for the 2010 Toyota Prius. The study estimated the instantons SOC using an EV energy consumption model [21]. The model estimates instantaneous energy consumption of EVs using vehicle operational data. The figure shows the instantaneous SOCs of two trips, the UDDS (or the city cycle) and the highway cycles. The study found that the model occasionally underestimated and overestimated the HEV regenerative energy. As shown in the figure, the estimated SOC generally follows the measured SOC trend of the test HEV. However, we found that the estimated SOC of the highway trip generally underestimated the electric energy consumption and predicted a higher final SOC. The results demonstrate that the SOC remains fairly stable throughout the trip.

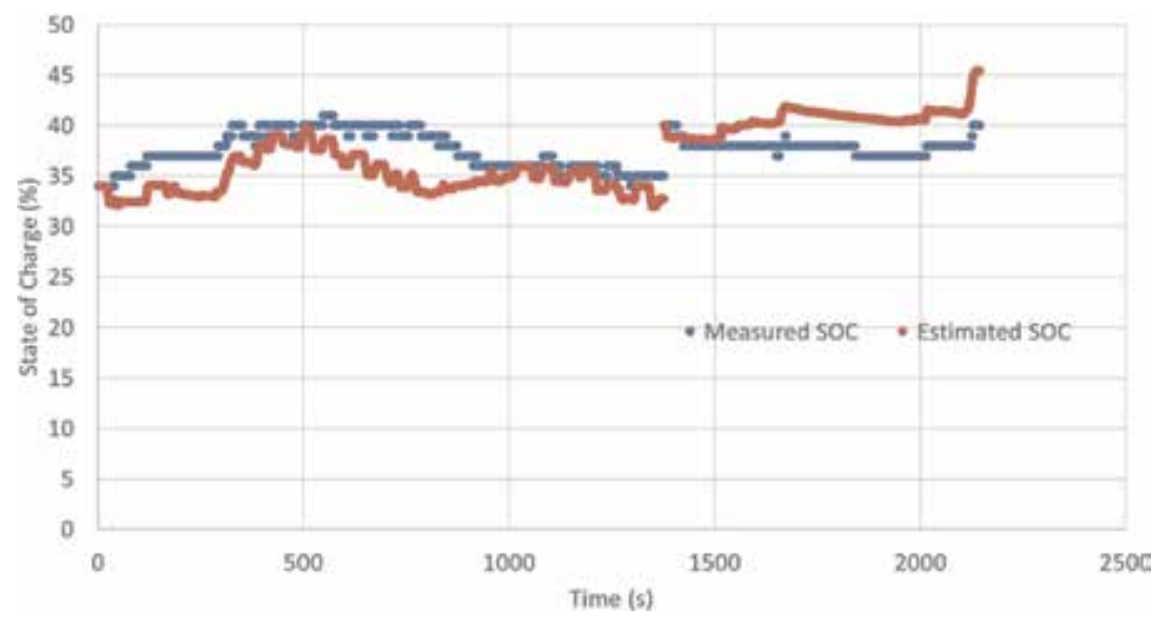

Figure 16.

The state of charge of $H E V$. 


\section{Conclusion}

This study developed a simple fuel consumption model for a 2010 Toyota Prius $\mathrm{HEV}$ that can be utilized for CV applications. The model was then generalized for other HEVs using a scale factor that is computed as the ratio of the vehicle EPA combined rating relative to the Toyota Prius rating. The proposed model utilizes instantaneous vehicle speed, acceleration, and roadway grade as input variables. The model estimates the vehicle fuel consumption accurately with an average error of $2.1 \%$ for the Toyota Prius and up to $4 \%$ for different HEVs. Although this simple model serves the desired purpose, further refinement of the model could be achieved by refining the EV model to reflect the variations of fuel consumption at low speeds and low engine power levels.

\section{Acknowledgements}

This work was funded by the Department of Energy through the Office of Energy Efficiency and Renewable Energy (EERE), Vehicle Technologies Office, Energy Efficient Mobility Systems Program under award number DE-EE0008209.

\section{Author details}

Kyoungho $\mathrm{Ahn}^{1}$ and Hesham A. Rakha ${ }^{2 *}$

1 Center for Sustainable Mobility, Virginia Tech Transportation Institute, Blacksburg, VA, United States

2 Department of Civil and Environmental Engineering and Center for Sustainable Mobility, Virginia Tech Transportation Institute, Blacksburg, VA, United States

*Address all correspondence to: hrakha@vt.edu

\section{IntechOpen}

(C) 2019 The Author(s). Licensee IntechOpen. This chapter is distributed under the terms of the Creative Commons Attribution License (http://creativecommons.org/licenses/ by/3.0), which permits unrestricted use, distribution, and reproduction in any medium, provided the original work is properly cited. (cc) BY 


\section{References}

[1] U.S. Energy Information

Administration (EIA). Monthly energy review. 2018. Available from: https:// www.eia.gov/totalenergy/data/month ly/pdf/mer.pdf [cited: June 5 2018]

[2] U.S. Energy Information Administration (EIA). Energy consumption by sector. 2014. Available from: http://www.eia.gov/totalenergy/ data/monthly/pdf/flow/css_2014_energ y.pdf [cited: June 30 2015]

[3] U.S. Energy Information Administration (EIA). Annual energy review energy perspectives. 2012. Available from: http://www.eia.gov/ todayinenergy/detail.cfm?id $=9250$ [cited: June 30 2015]

[4] U.S. Department of Energy. Hybrid and plug-in electric vehicles. 2018. Available from: https://www.afdc.energ y.gov/vehicles/electric.html [cited: June 30 2018]

[5] Rakha HA, Ahn K, Moran K, Saerens B, Van den Bulck E. Virginia tech comprehensive power-based fuel consumption model: Model development and testing. Transportation Research Part DTransport and Environment. 2011; 16(7):492-503

[6] Barsali S, Miulli C, Possenti A. A control strategy to minimize fuel consumption of series hybrid electric vehicles. IEEE Transactions on Energy Conversion. 2004;19(1):187-195

[7] Delprat S, Lauber J, Guerra T-M, Rimaux J. Control of a parallel hybrid powertrain: Optimal control. IEEE Transactions on Vehicular Technology. 2004;53(3):872-881

[8] Staunton RH, Ayers CW, Marlino L, Chiasson J, Burress B. Evaluation of 2004 Toyota Prius Hybrid Electric Drive System. Oak Ridge, TN (United States): Oak Ridge National Lab. (ORNL); 2006
[9] Xiong W, Zhang Y, Yin C. Optimal energy management for a series-parallel hybrid electric bus. Energy Conversion and Management. 2009;50(7):1730-1738

[10] Mansour C, Clodic D. Dynamic modeling of the electro-mechanical configuration of the Toyota hybrid system series/parallel power train. International Journal of Automotive Technology. 2012;13(1):143-166

[11] Lin C-C, Peng H, Grizzle JW, Kang J-M. Power management strategy for a parallel hybrid electric truck. IEEE Transactions on Control Systems Technology. 2003;11(6):839-849

[12] Gao J, Zhu GG, Strangas EG, Sun F. Equivalent fuel consumption optimal control of a series hybrid electric vehicle. Proceedings of the Institution of Mechanical Engineers, Part D: Journal of Automobile Engineering. 2009;223(8): 1003-1018

[13] Bowles P, Peng H, Zhang X. Energy management in a parallel hybrid electric vehicle with a continuously variable transmission. In: Proceedings of the 2000 American Control Conference; IEEE; 2000

[14] Fontaras G, Pistikopoulos P, Samaras Z. Experimental evaluation of hybrid vehicle fuel economy and pollutant emissions over real-world simulation driving cycles. Atmospheric Environment. 2008;42(18):4023-4035

[15] Al-Samari A. Study of emissions and fuel economy for parallel hybrid versus conventional vehicles on real world and standard driving cycles. Alexandria Engineering Journal. 2017;56:721-726

[16] Thomas J, Huff S, West B, Chambon P. Fuel consumption sensitivity of conventional and hybrid electric light-duty gasoline vehicles to 
driving style. SAE International Journal of Fuels and Lubricants. 2017;10(3):

672-689

[17] Wipke KB, Cuddy MR, Burch SD.

ADVISOR 2.1: A user-friendly advanced powertrain simulation using a combined backward/forward approach. IEEE Transactions on Vehicular Technology. 1999;48(6):1751-1761

[18] Boubaker S, Rehimi F, Kalboussi A. Estimating energy consumption of hybrid electric vehicle and Gazoline classical vehicle. In: 2013 International Conference on Advanced Logistics and Transport (ICALT); 2013. pp. 221-226

[19] Argonne National Laboratory. 2010 Toyota Prius. 2017. Available from: http://www.anl.gov/energy-systems/g roup/downloadable-dynamometer-da tabase/hybrid-electric-vehicles/ 2010-toyota-prius [cited: March 27 2018]

[20] Rakha H, Ahn K, Trani A. Development of VT-micro model for estimating hot stabilized light duty vehicle and truck emissions.

Transportation Research Part D: Transport and Environment. 2004;9(1): 49-74

[21] Fiori C, Ahn K, Rakha HA. Powerbased electric vehicle energy consumption model: Model development and validation. Applied Energy. 2016;168:257-268 



\title{
Driving Control Technologies of New High-Efficient Motors
}

\author{
Chang-Ming Liaw, Jia-Hsiang Zhuang, Shih-Wei Su, \\ Kai-Wei Hu and G. Vijay Kumar
}

\begin{abstract}
Although induction motor is the most commonly used actuator in the industry, its efficiencies are difficult to upgrade due to the existence of rotor copper losses. Permanent-magnet synchronous motor (PMSM) and synchronous reluctance motor (SynRM) are the two new motors being able to achieve the IE4-class and even the IE5-class efficiency. As to the switched reluctance motor (SRM), it lacks commercialized motors and power modules being available. For establishing highperformance PMSM and SynRM drives, many key affairs must be properly treated, which are introduced in this article. The contents presented in this chapter include: (1) interdisciplinary affairs of motor drives, (2) comparative characteristics and applications of commonly used motors, (3) reversible and generator operations for specific motors, (4) power electronic converters for motor drives, (5) possible new high-efficient motor drives, (6) PMSM drives, (7) SynRM drives, (8) some key issues of PMSM drives, (9) some key issues of SynRM drives, (10) example of PMSM drive, and (11) example of SynRM drive.
\end{abstract}

Keywords: PMSM, SynRM, dynamic modeling, commutation, current control, speed control, efficiency

\section{Introduction}

Induction motors [1-3] are still the most popularly applied motor owing to their simple structures, ease of operation, and mature driving technologies. However, their best efficiencies are generally still in IE3-class due to the existence of rotor copper losses. Upgrading the efficiencies of IMs to IE4-class via using high-grade core and rotor cage materials will not be cost-effective. Permanent-magnet synchronous motor (PMSM) and synchronous reluctance motor (SynRM) [2-6] are the two new motors being able to achieve the IE4-class and even the IE5-class efficiency. As to the switched reluctance motor (SRM) [7], although it may also possess the potential, it still lacks commercialized motors and power modules being available.

To establish a high-performance PMSM drive, the proper match between the available source, the interface converter, the motor drive, and the mechanical load must be made. Some major affairs to be properly treated include: (i) physical modeling and parameter estimation [8, 9]; (ii) current control [10-13]: generally, the current control methods can be categorized into hysteresis controls, rampcomparison controls and predictive controls; (iii) speed control [14-17]; (iv) direct torque control based on space-vector PWM [18]; (v) commutation shift and 
field-weakening control [19-25]: through appropriate d-axis field current setting or commutation shift, the developed torque can be increased by utilizing the reluctance torque component effectively. In the case of constant power, it can extend the speed range through field-weakening; (vi) voltage boosting [26-28]: for a battery-powered EV PMSM drive, the equipped battery followed interface DC/DC converter providing adjustable DC-link voltage can increase the battery voltage selection flexibility and the overall drive system rating utilization. The buck-boost bidirectional interface converters are adopted in the PMSM motor drive [27]. It can step down the voltage from battery; hence the lower DC-link voltage under lower speed can improve the efficiency. As the motor drive is drawn from the mains, the single-phase or three-phase switch-mode rectifiers (SMRs) [29-32] must be equipped to achieve the DC-link voltage boosting task.

For a standard PMSM drive, the rotor absolute position is necessary to make its vector control. However, for avoiding the risk of sensor failure, the position sensorless controlled motor drive is preferable. Generally, the existing PMSM position sensorless control approaches can be classified into [33-35]: (i) methods based on the measured and/or identified machine parameters [36,37]; (ii) methods based on rotor saliency and magnet anisotropy: the high-frequency injection approaches are the typical ones. The high-frequency sinusoidal-wave [38-40] or square-wave [41, 42] voltage with suited amplitude and frequency is injected into the studied motor, and the detected current is processed to yield the observed rotor position. These approaches can be successfully operated under low speed including standstill, and no machine parameters are needed. However, the injected signal will yield high-frequency noise, lower efficiency and the back-EMF harmonics in IPMSM may cause the estimated rotor position error; (iii) observer based methods [36, 43, 44]: these include adaptive observers, sliding mode observers, Kalman filter observers and reduced-order observers, etc.; (iv) back-EMF methods [45-47]: these methods are obviously not suitable for EV due to its frequent accelerating/decelerating operations; and (v) hybrid methods [48, 49].

As generally recognized, PMSM possesses high power density and efficiency. However, its manufacture process is complicated with higher cost. And it has the demagnetization risk. As to the synchronous reluctance motor (SynRM), although it has no permanent magnet torque component, good performance/cost-compromised performance can still be obtained if the proper switching control is made. It possesses high application potential, especially for high-speed driving, since its rotor is rigid and cogging torque free from having no permanent magnets and conductors.

Since only some air slots are existed in the rotor of a SynRM, its energy conversion characteristics are significantly affected by rotor geometry [50-52]. The rotor is assembled with stacked silicon steels, which leads the rotor to be easily saturated, and the iron loss is comparatively higher than PMSM. Hence, considering iron loss and magnetic saturation is necessary for achieving high performance and efficiency.

In efficiency optimization of a SynRM, both the copper loss and the iron loss must be considered. The copper loss is load-dependent, while the iron loss is related to the air-gap flux level. Basically, loss minimization approaches [53-62] for SynRM can be roughly categorized into loss model-based control and searching control. The former approaches minimize the power consumption by setting the optimal d-axis current command, which is derived from the loss function using machine parameters. Obviously, the resulted control performance is affected by the motor parameter variations, especially the d-axis inductance. As to the searching methods, their performances are insensitive to motor parameter variations. However, the complicated searching process usually causes the sluggish dynamic response, torque ripple, and additional losses. Finally, for illustrating the key technological affairs introduced in this article, the example battery/supercapacitor powered EV PMSM drive [63] and SMR-fed SynRM drive $[64,65]$ are presented in Section 5 and Section 6. 


\section{Basic motor drive system configuration}

As shown in Figure 1, motor drive is an interdisciplinary mechatronic system including motor, mechanical load, power converter, control scheme, transducing, and sensing schemes. The proper design of motor and the proper match between system-constituted components should be made for yielding good driving performance.

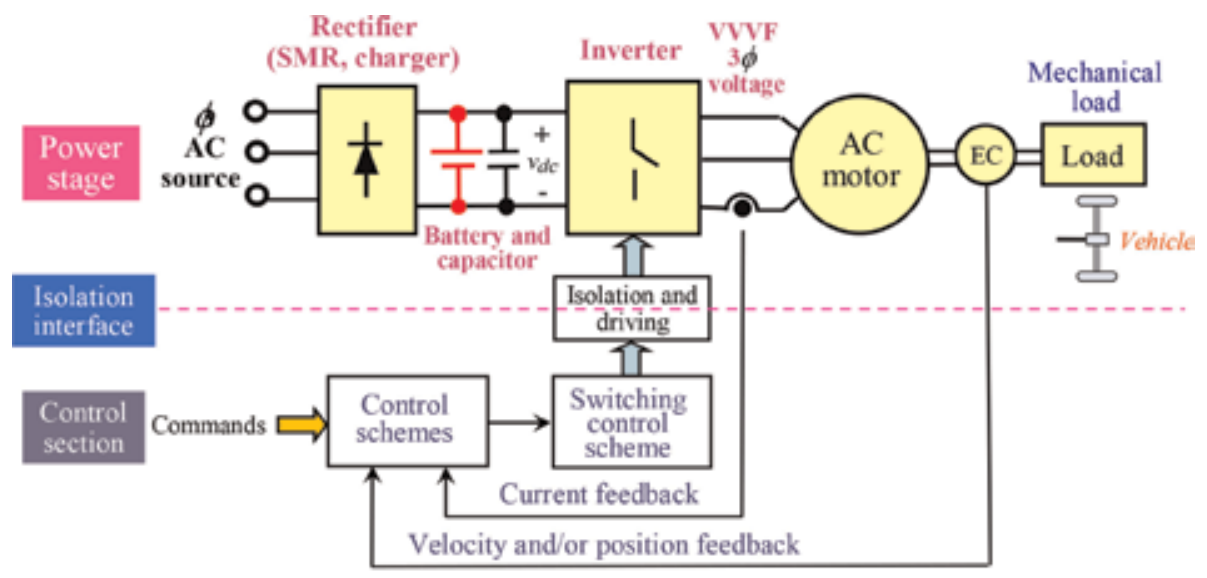

Requirements: Reliable, low cost, miniaturization (smaller volume and weight), higher efficiency (energy saving), low vibration and acoustic noise, etc.

Figure 1.

Typical motor drive system configuration.

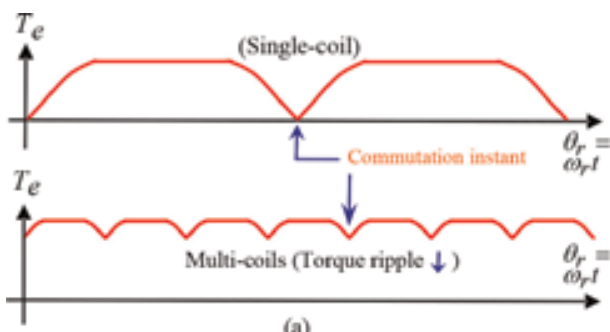

(a)

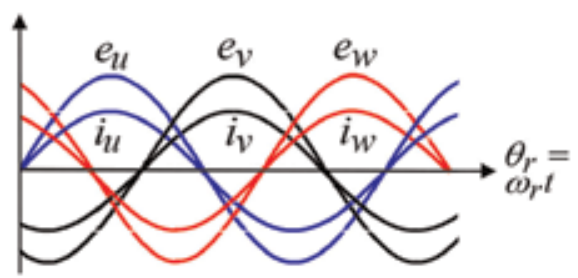

$P_{m}=e_{u} i_{u}+e_{v} i_{v}+e_{w} i_{w}=$ constant $=T_{e} \omega_{r}$
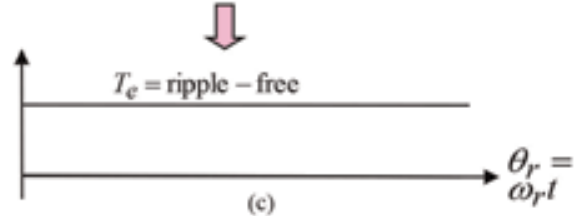

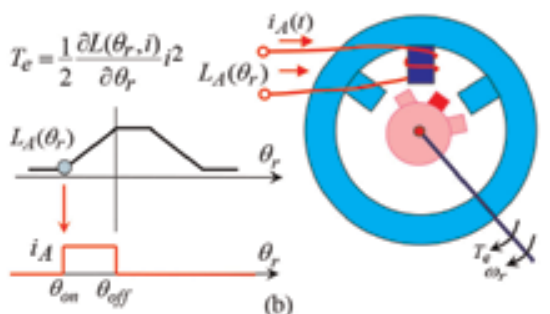

(b)

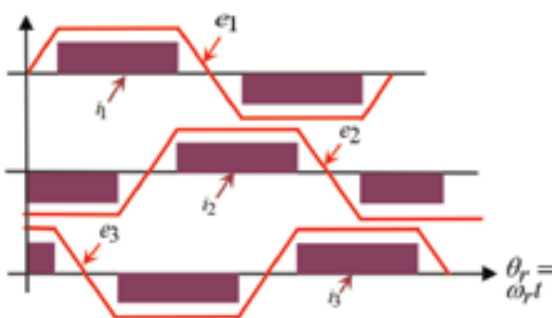

$P_{m}=\Sigma e_{k} i_{k}=T_{e} \omega_{r}=$ constant $\Rightarrow T_{e}=$ constant

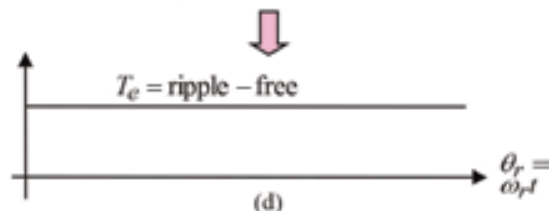

(d)

Figure 2.

Sketched ideal developed torque characteristics of some motors: (a) DCM, (b) SRM, (c) three-phase sine-wave PMSM (d) three-phase square-wave PMSM. 
The commonly used motors include brushed DC motor (DCM), induction motor (IM), and synchronous motor (SM) consisting of permanent-magnet synchronous motor (PMSM), synchronous reluctance motor (SynRM), switched reluctance motor (SRM), etc. The ideal developed torque characteristics of some typical motors are sketched in Figure 2. In reality, each type of motor possesses its distinct affairs, which must be properly treated.

\section{Permanent-magnet synchronous motors}

\subsection{Structural features of synchronous motors}

Except for the slot-less stator, the slotted and salient-pole stators are shown in Figure $3 \mathbf{a}$ and $\mathbf{b}$. The former is mainly employed for forming distributed armature winding with sinusoidal currents. It is suitable for low-speed driving applications because it generates smoother developed torque. The salient-pole stator in Figure $3 \mathbf{b}$ is used for concentrated windings. This winding has higher efficiency owing to its lower copper losses. However, it possesses higher torque ripple and thus only suited for speed driving applications.

Some rotor structures of SM are sketched in Figure 4a-g. The SPMSM shown in Figure 4a possesses less cogging torque and smoother developed torque. Hence, it is suitable for low-speed driving applications. In order to increase the rigidity, one can adopt the inset SPMSM shown in Figure $\mathbf{4 b}$. There are many types of interior PMSM (IPMSM) with various shapes of PM. The two typical rotor structures of IPMSM with buried and interior magnets are shown in Figure $4 \mathbf{c}$ and $\mathbf{d}$. The existence of saliency allows them to produce reluctance torque in addition to electromagnetic torque. And the field weakening is simpler to achieve. For higher speed driving applications, one can adopt the permanent-magnet-assisted SynRM (PMaSynRM) shown in Figure 4e. Two types of SynRM rotor structure are shown in Figure $\mathbf{4 f}$ and $\mathbf{g}$, namely, the axially laminated anisotropy (ALA) rotor and the transversally laminated anisotropy (TLA) rotor.

Comments: SynRM belongs to SM having: (i) distributed armature windings; and (ii) PM-free rotor with air slots. As to the switched reluctance motor (SRM), its features lie in: (i) concentrated armature windings and (ii) teethed rotor. It follows that these two reluctance machines have the major comparative features: (i) they all have only reluctance torque, and the developed torque or power is highly affected

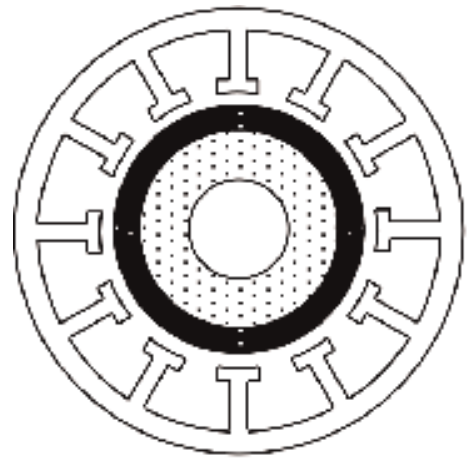

(a)

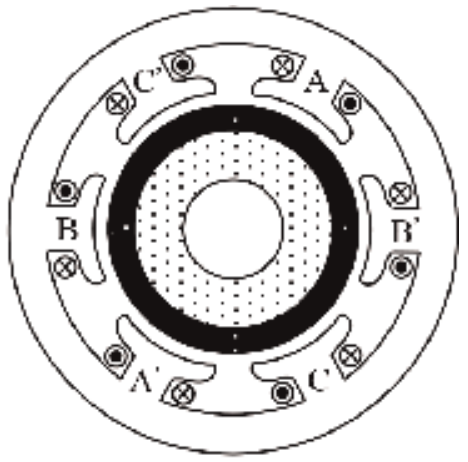

(b)

Figure 3.

Typical stator structures of synchronous motors: (a) slotted and (b) salient-pole. 


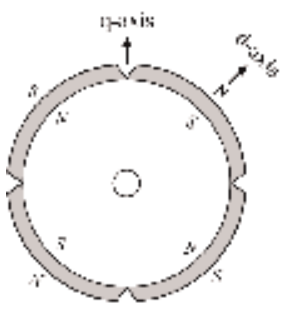

(a)

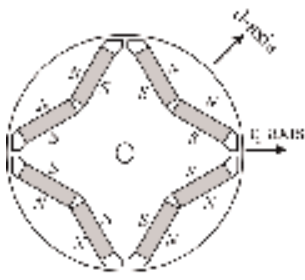

(L)

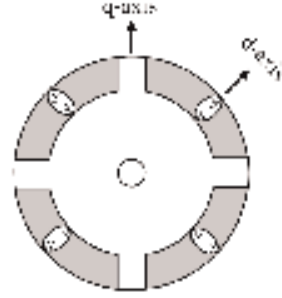

(b)

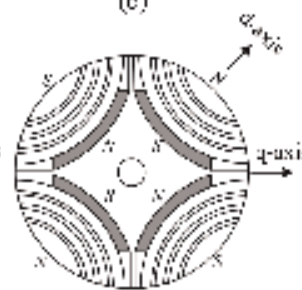

iा

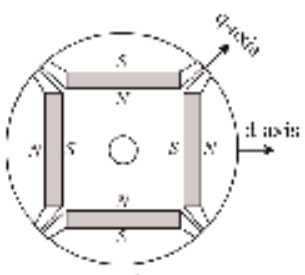

ick

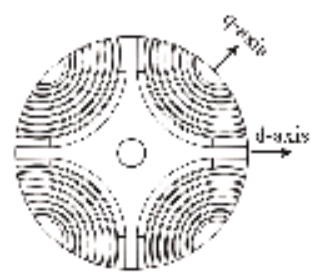

(j)

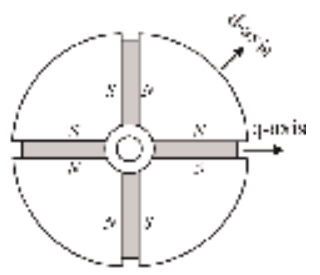

(d)

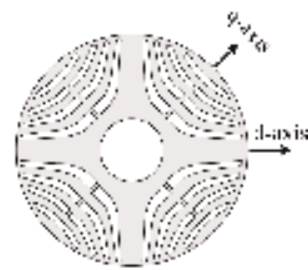

(b)

Figure 4 .

Some typical rotor structures of synchronous motors: (a) SPMSM, (b) inset SPMSM, (c) radial IPMSM, (d) tangential IPMSM, (e) hybrid V-shape IPMSM, (f) PMASynRM, (g) ALA SynRM, and (h) TLA SynRM.

by commutation instant setting and (ii) although they all possess high ripple torques under low speed, the one yielded by SynRM is less owing to distributed armature with sinusoidal current excitation.

\subsection{Physical modeling of PMSM}

\subsubsection{Voltage equations}

Figure 5 shows the configuration of a Y-connected three-phase 2-pole PMSM. The Y-connected resistors across the armature terminals are used to estimate the motor phase back-EMF under no-load, that is, $v_{a n^{\prime}} \approx e_{a s}$. The following assumptions are made for making analytic derivation: (i) symmetrical and sinusoidally distributed three-phase armature windings, (ii) sinusoidal armature back-EMFs, and (iii) linear magnet circuit. And the rotor position $\theta_{r}$ is defined as the angle between asaxis and the sensed q-axis.

The phase (a-phase as an example, the ones for the other two phases, can be written analogously) voltage equation can be expressed as:
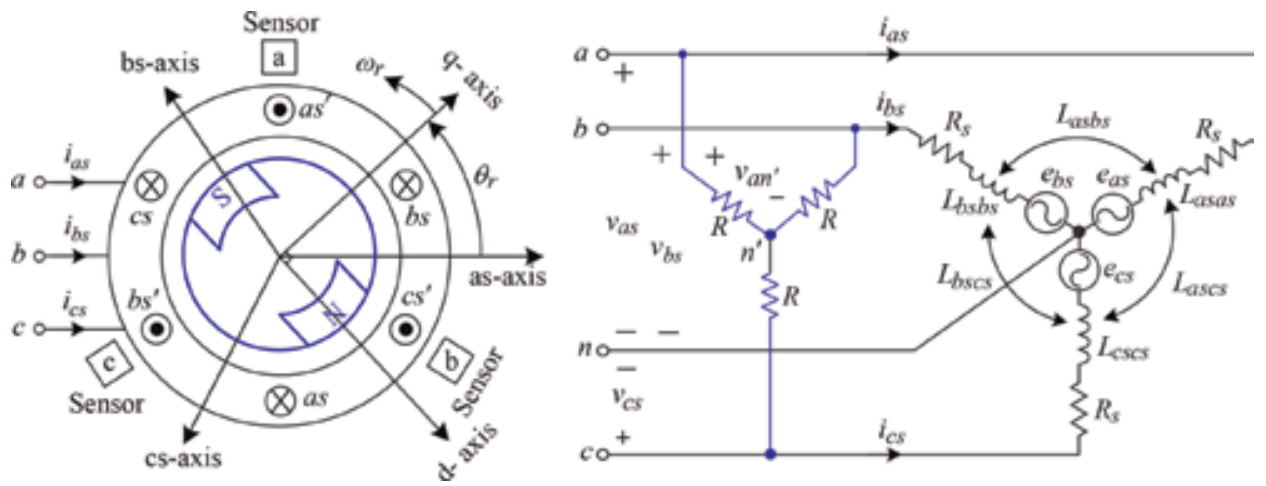

Figure 5.

Configuration of an elementary three-phase two-pole Y-connected PMSM. 


$$
\begin{gathered}
v_{a s}=R_{s} i_{a s}+\frac{d}{d t} \lambda_{a s}=R_{s} i_{a s}+\frac{d}{d t}\left(L_{a s a s} i_{a s}+L_{a s b s} i_{b s}+L_{a s c s} i_{c s}\right)+e_{a s} \\
L_{a s a s}=L_{l s}+L_{A}+L_{B} \cos 2 \theta_{r} \\
e_{a s}=\lambda_{m}^{r} \omega_{r} \cos \theta_{r} \stackrel{\Delta}{=} \hat{E}_{a s} \cos \theta_{r} \\
\theta_{r}=\theta_{r}(0)+\int_{0}^{t} \omega_{r}(\zeta) d \zeta
\end{gathered}
$$

where $v_{a s}$ is winding terminal voltages, $i_{a s}$ is winding current, $R_{s}$ is winding resistance, $\lambda_{a s}$ is winding flux linkage, $\lambda_{m}^{r}$ is peak flux linkage contributed by the rotor permanent magnet, $L_{a s a s}$ is winding self-inductance, $L_{a s b s}$ is mutual inductance, $L_{l s}$ is winding leakage inductance, $\theta_{r}$ is rotor electrical angular position, $\theta_{r}(0)$ is the initial position of q-axis relative to a-axis, $\omega_{r}$ is rotor electrical angular speed, $e_{a s}$ is back electromotive force (EMF), and $\hat{E}_{a s}$ is the peak value of $e_{a s}$.

By applying rotor rotating frame transformation, the voltage equations in dq-frame can be written as:

$$
\begin{gathered}
v_{q s}=\left(R_{s}+p L_{q}\right) i_{q s}+\omega_{r} L_{d} i_{d s}+\omega_{r} \lambda_{m}^{\prime r} \\
v_{d s}=\left(R_{s}+p L_{d}\right) i_{d s}-\omega_{r} L_{q} i_{q s} \\
L_{q}=L_{l s}+L_{m q}=L_{l s}+\frac{3}{2}\left(L_{A}+L_{B}\right) \\
L_{d}=L_{l s}+L_{m d}=L_{l s}+\frac{3}{2}\left(L_{A}-L_{B}\right)
\end{gathered}
$$

where $L_{q}\left(L_{d}\right)$ denotes q-axis (d-axis) inductance and $L_{q}>L_{d}$.

\subsubsection{Torque and mechanical equations}

The electromagnetic developed torque and mechanical equations of a PMSM drive in dq-frame can be expressed as:

$$
T_{e}=\frac{3 P}{4}\left[\lambda_{m}^{\prime r} i_{q s}+\left(L_{d}-L_{q}\right) i_{q s} i_{d s}\right]=\frac{3 P}{4}\left[\lambda_{m}^{\prime} \hat{I}_{a s} \cos \beta+\frac{L_{d}-L_{q}}{2} \hat{I}_{a s}^{2} \sin 2 \beta\right]=T_{L}+B \omega_{r}+J \frac{d \omega_{r}}{d t}
$$

where $P$ is pole number, $\hat{I}_{a s}$ is peak of a-phase current, the variables $\beta$ denotes the shift angle between q-axis and peak of a-phase current, $T_{L}$ is load torque, $B$ is total damping coefficient, and $J$ is total inertia constant.

\subsection{Measurement of motor key parameters}

Before establishing the motor drive, some key motor parameters must be measured to comprehend the characteristics of the studied motor. Figure 6 shows the experimental mechanism for conducting the measurements.

\subsubsection{Winding resistance and inductances}

For the Y-connected PMSM with isolated neutral as indicated in Figures $\mathbf{5}$ and $\mathbf{6}$, from Eq. (1), the inductance and resistance of PMSM between a-phase and b-phase can be expressed as: 


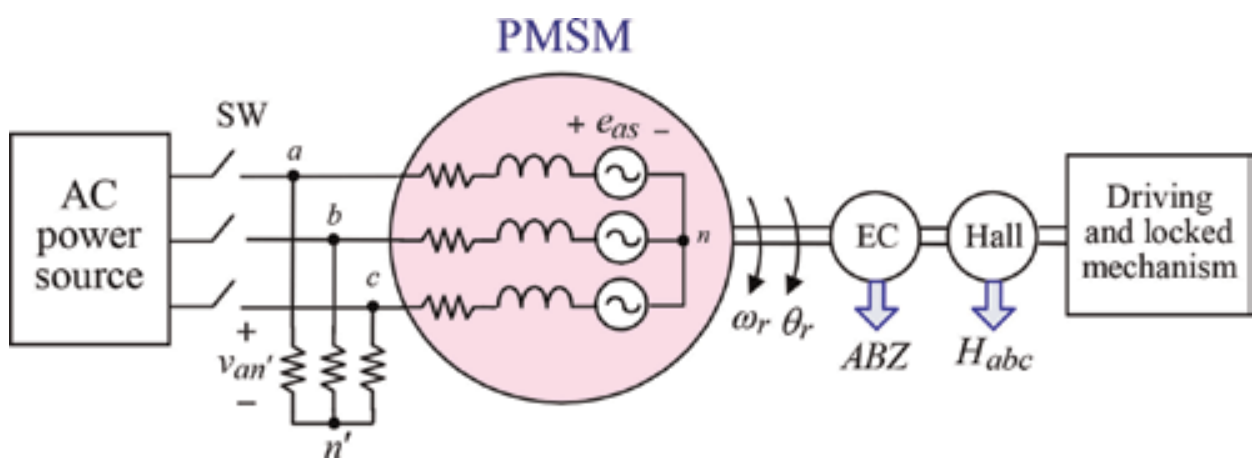

Figure 6.

Parameter measurement mechanism for SMs.

$$
R_{a b}=2 R_{s}, \quad L_{a b}\left(\theta_{r}\right)=2 L_{l s}+3 L_{A}-3 L_{B} \cos \left(2 \theta_{r}-\frac{2 \pi}{3}\right)
$$

And the q-axis and d-axis inductances of Eqs. (7) and (8) can be expressed from Eq. (10) as:

$$
\begin{gathered}
L_{q}=\frac{L_{a s b s, \max }}{2}=\frac{1}{2} L_{a s b s}\left(\theta_{r}=-\frac{\pi}{6}\right)=L_{l s}+\frac{3}{2} L_{A}+\frac{3}{2} L_{B} \\
L_{d}=\frac{L_{a s b s, \min }}{2}=\frac{1}{2} L_{a s b s}\left(\theta_{r}=\frac{\pi}{3}\right)=L_{l s}+\frac{3}{2} L_{A}-\frac{3}{2} L_{B}
\end{gathered}
$$

A. Winding resistance: the armature winding resistance $R_{s}=R_{a b} / 2$ can be estimated by DC excitation method.

B. Winding inductances: the $L_{a b}\left(\theta_{r}\right)$ can be measured using the LCR meter under various frequencies at different rotor positions. Then the q-axis and d-axis inductances $L_{q}$ and $L_{d}$ can be obtained from Eqs. (11) and (12). However, the inductance-saturated effects cannot be acquired for the small-signal excitation. On the other hand, one can apply the step-response method under various current levels.

\subsubsection{Back-EMF and PM flux linkage $\lambda_{m}^{\prime}$}

As shown in Figure 5, three $\mathrm{Y}$-connected resistors with a reasonably high resistance $(R=100 \mathrm{k} \Omega)$ are connected at the stator terminals to observe the motor phase back-EMF under no-load. By letting $i_{a s}=i_{b s}=i_{c s}=0$, the back-EMF can be found from Eqs. (1) and (3) as:

$$
e_{a s}=\hat{E}_{a s} \cos \theta_{r}=\left.\left.v_{a s}\right|_{\mathrm{NL}} \approx v_{a n^{\prime}}\right|_{\mathrm{NL}}
$$

And $\lambda_{m}^{\prime r}$ can be obtained from Eq. (3) as:

$$
\lambda_{m}^{\prime r}=\frac{\hat{E}_{a s}}{P / 2 \times \omega_{r}}(\mathrm{~Wb})
$$

\subsubsection{Measured examples}

A. Figure $7 \mathbf{a}$ and $\mathbf{b}$ depict the measured back-EMFs and their spectra of two examples of PMSMs: (a) under $1000 \mathrm{rpm}$ of a three-phase SPMSM, $5 \mathrm{~kW}$, 
24.5 N-m, 8-pole, $2000 \mathrm{rpm}$ and (b) under $1000 \mathrm{rpm}$ of a three-phase IPMSM, $1 \mathrm{~kW}, 3.23 \mathrm{~N}-\mathrm{m}, 6$-pole, $3000 \mathrm{rpm}$.

B. Observations: for the IPMSM, the distorted back-EMF waveform with eleventh and thirteenth orders are observed. The influences of back-EMF harmonics on the current control and the high-frequency-injected position sensorless control must be considered.

\subsection{Some key issues}

Figure 8 shows some key issues affecting the performance of PMSM and SynRM drives. The typical ones include: (i) suitable motor type selection, (ii) motor parameters and dynamic model estimation, (iii) commutation instant setting and shifting, (iv) inverter and its PWM switching control, (v) field weakening, (vi) DClink voltage boosting, (vii) regenerative braking operation, (viii) generator operation, and (ix) position sensorless control. Some comments are given below:

A. Motor selection: the stator and rotor structural features of some SMs have been explored in Section 3.1. The comparative loss and efficiency characteristics of some PMSMs and SynRM are depicted in Figure 9 [2, 3]. Basically, as the speed is increased, the PMSM with fewer permanent magnets is chosen for the ease of conducting field-weakening control. And finally, the SynRM or SRM is employed for higher speed driving applications.

B. Commutation: from Eqs. (3)-(9), one can be aware that the back-EMF and the developed torque of PMSM are highly affected by the speed, load, and commutation angle. The commutation shifting angle $\beta$ must be properly set and tuned to yield better developed torque. Commutation-advanced shift possesses field-weakening effects to reduce the back-EMF effects on the winding current response. It is worth noting that the commutation shift can

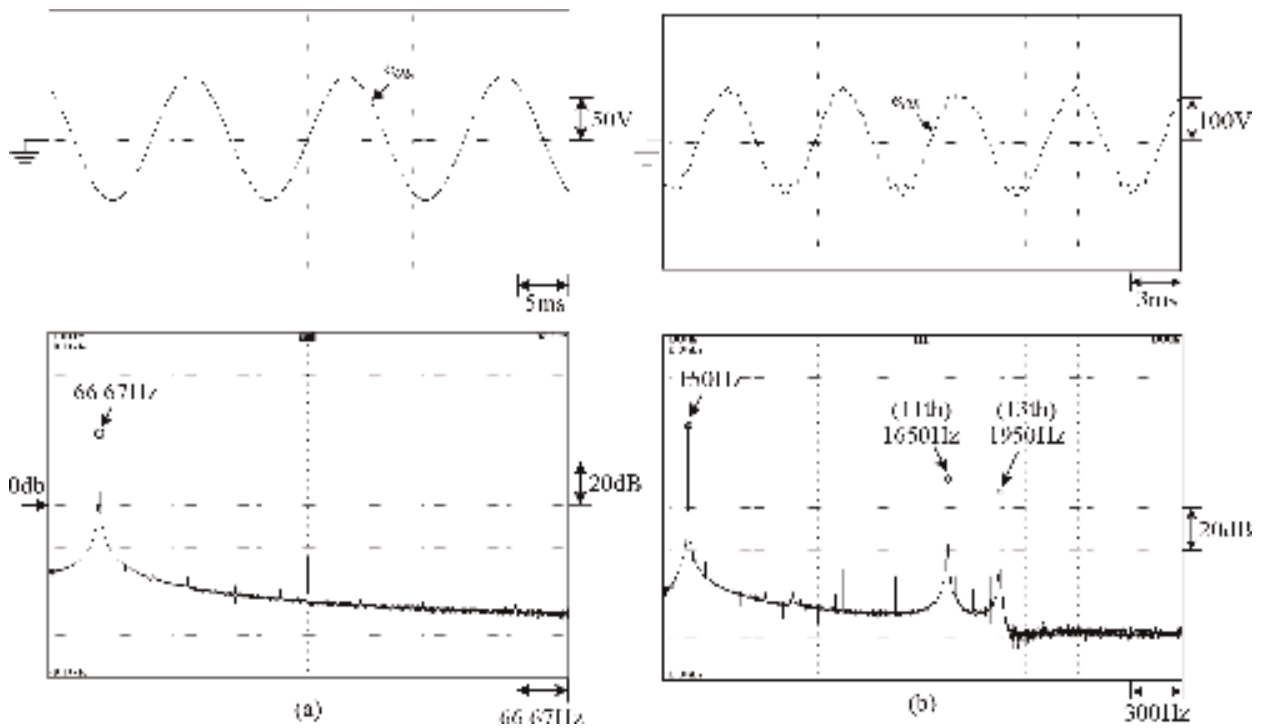

Figure 7.

Measured back-EMFs and their spectra of two examples of PMSMs: (a) under 1000 rpm of a three-phase SPMSM, $5 \mathrm{~kW}, 24.5 \mathrm{~N} \mathrm{~m}, 8$-pole, $2000 \mathrm{rpm}$ and (b) under $1000 \mathrm{rpm}$ of a three-phase IPMSM, $1 \mathrm{~kW}$, $3.23 \mathrm{Nm}$, 6-pole, $3000 \mathrm{rpm}$. 


\section{Some Key Issues of Synchronous Motor Drives (PMSM and SynRM)}

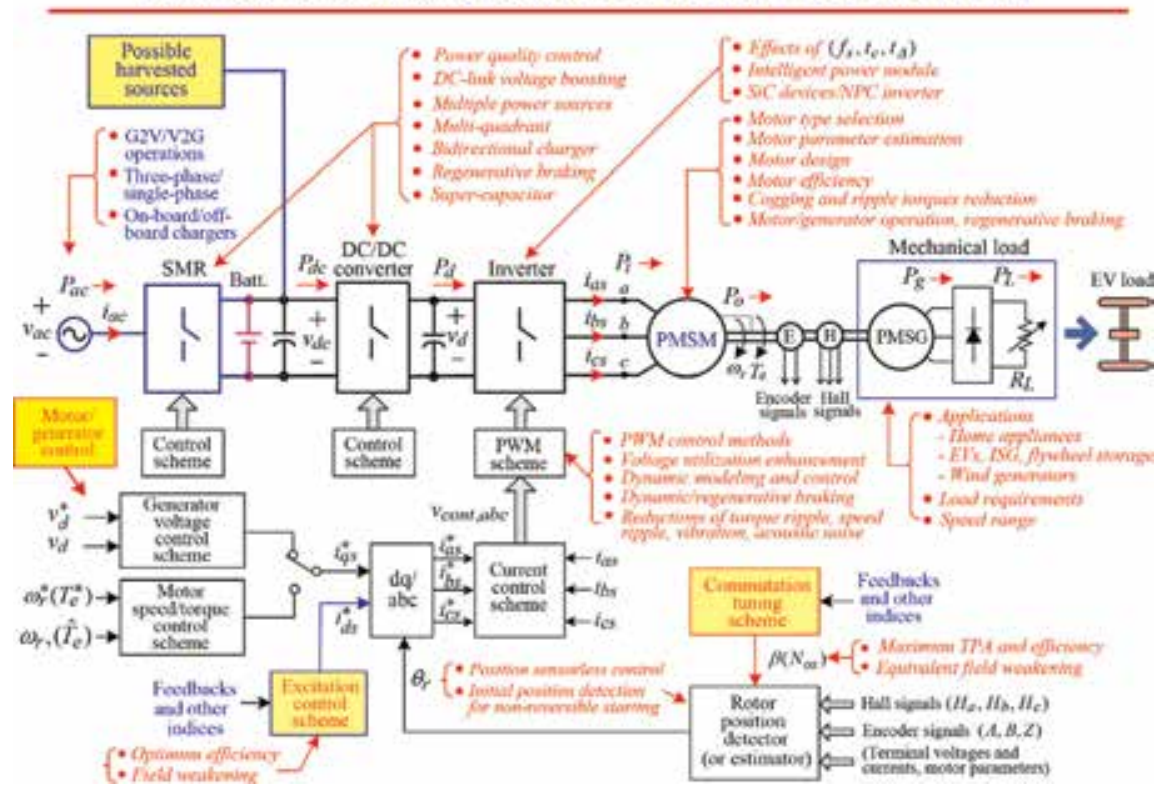

Figure 8.

Some key issues of PMSM and SynRM drives.
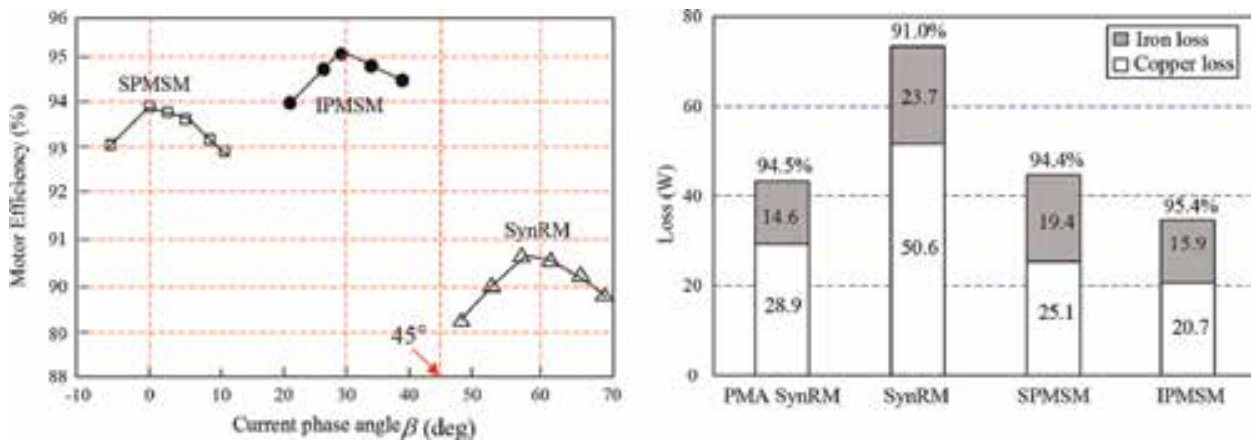

Figure 9.

Comparative efficiencies of some typical synchronous motors.

also be equivalently achieved via directly setting the d-axis field current command. Basically, the commutation advanced shifting angles for various SMs are (i) SPMSM, $\beta=0^{\circ}$; (ii) SynRM, $\beta=45^{\circ}$ theoretically; and (iii) IPMSM and PMASynRM, $\beta=0 \sim 45^{\circ}$. This fact can also be observed from Figure 9.

C. Current control and effects of current ripple: for an inverter-fed AC motor drive, its driving characteristics are greatly affected by the adopted PWM approach, including fundamental frequency control and harmonic spectral features. The current-controlled PWM (CCPWM) scheme is normally applied for high-performance motor drive. As indicated in Figure 10a and $\mathbf{b}$, the CCPWM scheme can be realized in abc-domain or dq-domain.

D. In some special application cases, the SPMSM is still adopted as the actuator for very high-speed driving applications with very compact volume. In these cases, the accurate commutation instant setting is very critical for avoiding 
magnet demagnetization, especially for position sensorless controlled drive. Moreover, the suited filtering chokes must be inserted between the inverter and the motor, as indicated in Figure 11.

E. Voltage boosting: under high speed, the sufficiently high back-EMF may make the winding current tracking performance sluggish as indicated in Figure 11 and thus yield the worsened developed torque. Voltage boosting is the effective means [26-28] to solve these problems. To accomplish this goal, one must equip the suited SMR or DC-DC interface converter for the motor drive being powered from the three-phase/single-phase mains or the battery. Some typical schematic arrangements of the bilateral SMRs and DC-DC interface converters are shown in Figures 12 and 13.

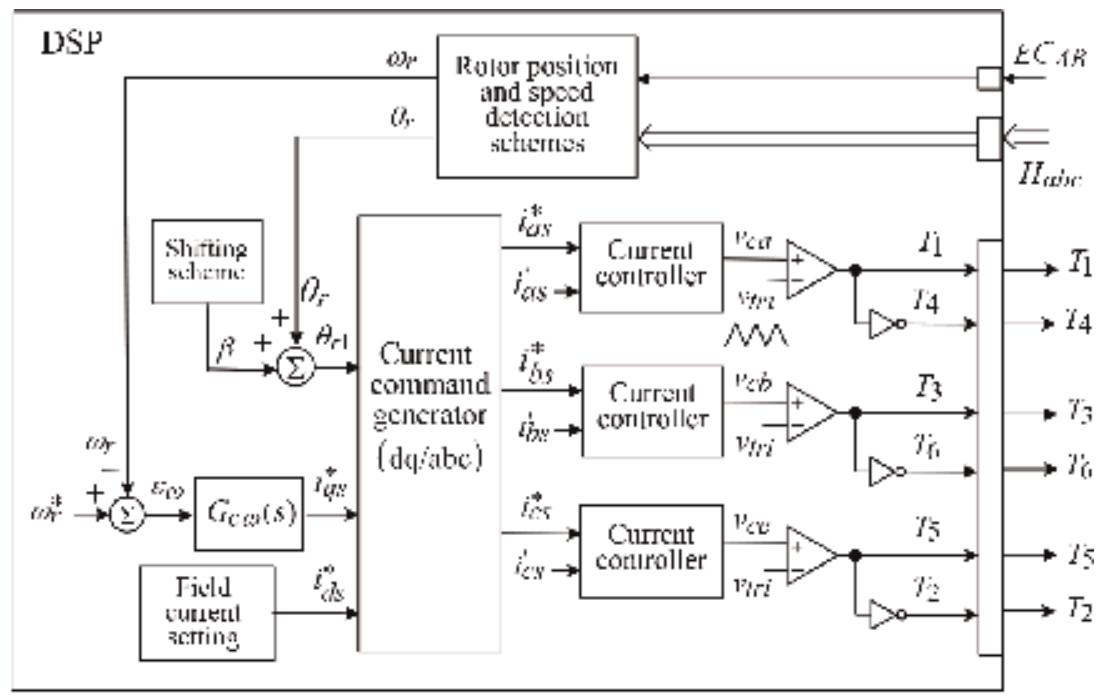

(a)

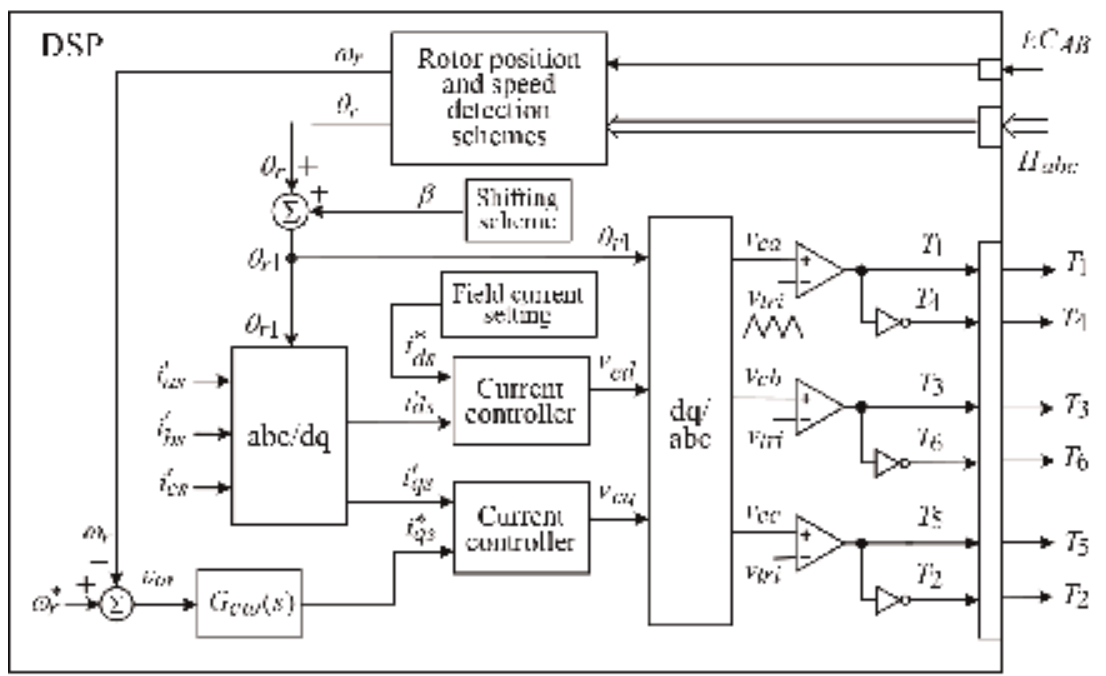

(b)

Figure 10.

Configurations of CCPWM schemes in (a) abc-domain and (b) dq-domain. 


\section{Effects of non-ideal current waveforms}

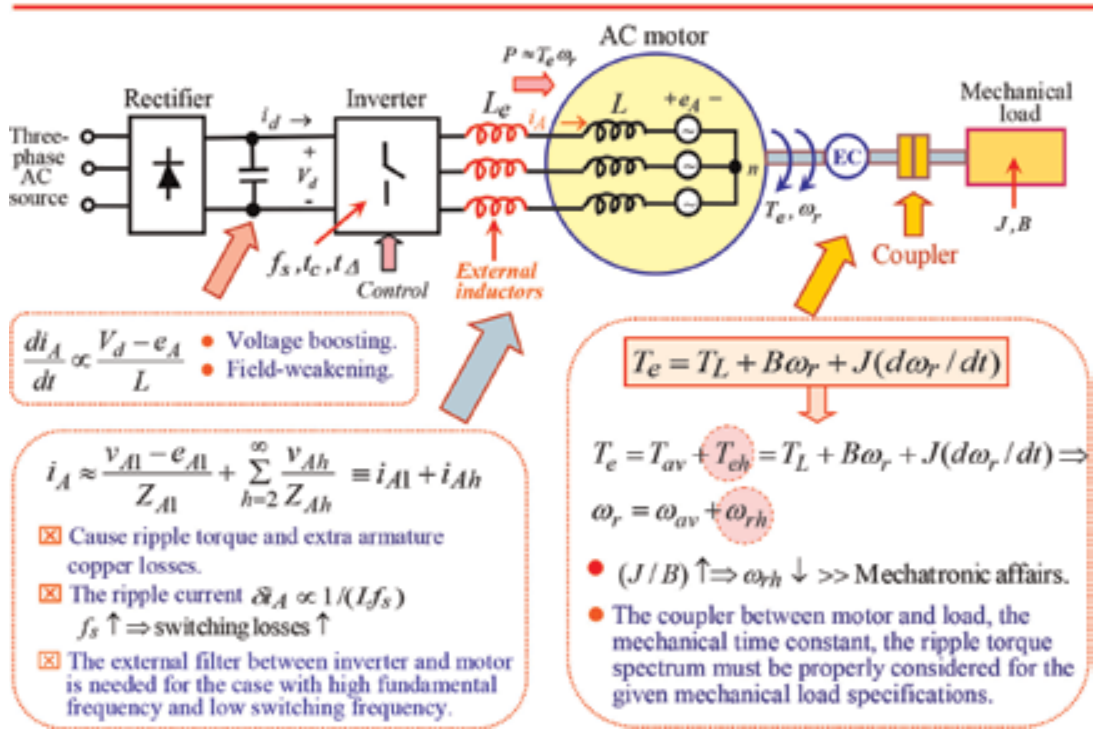

Figure 11.

Effects of DC-link voltage level and non-ideal winding current waveforms on the AC motor drive driving characteristics.

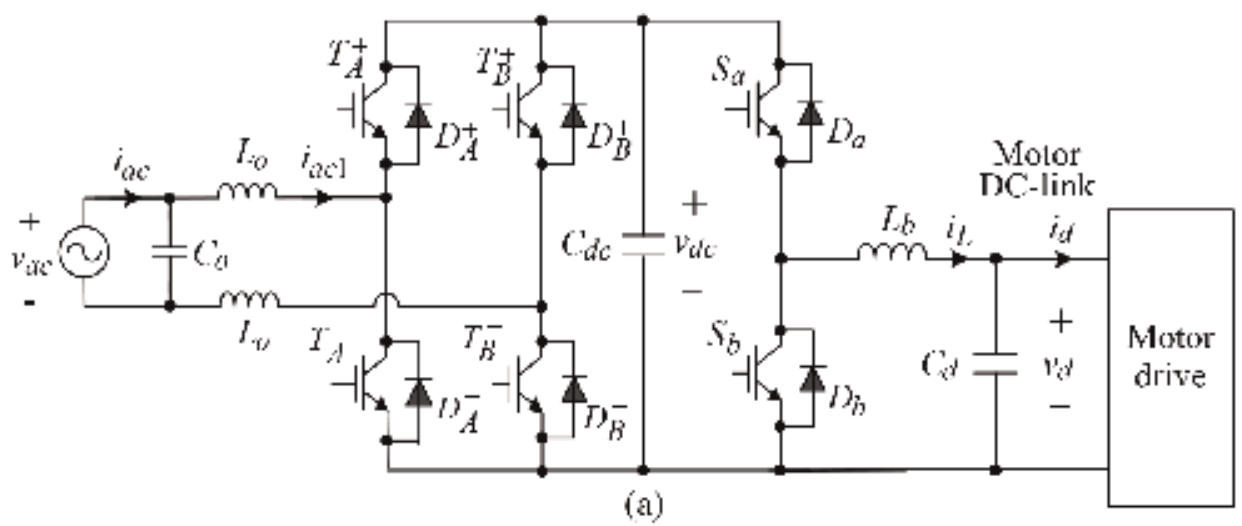

(a)

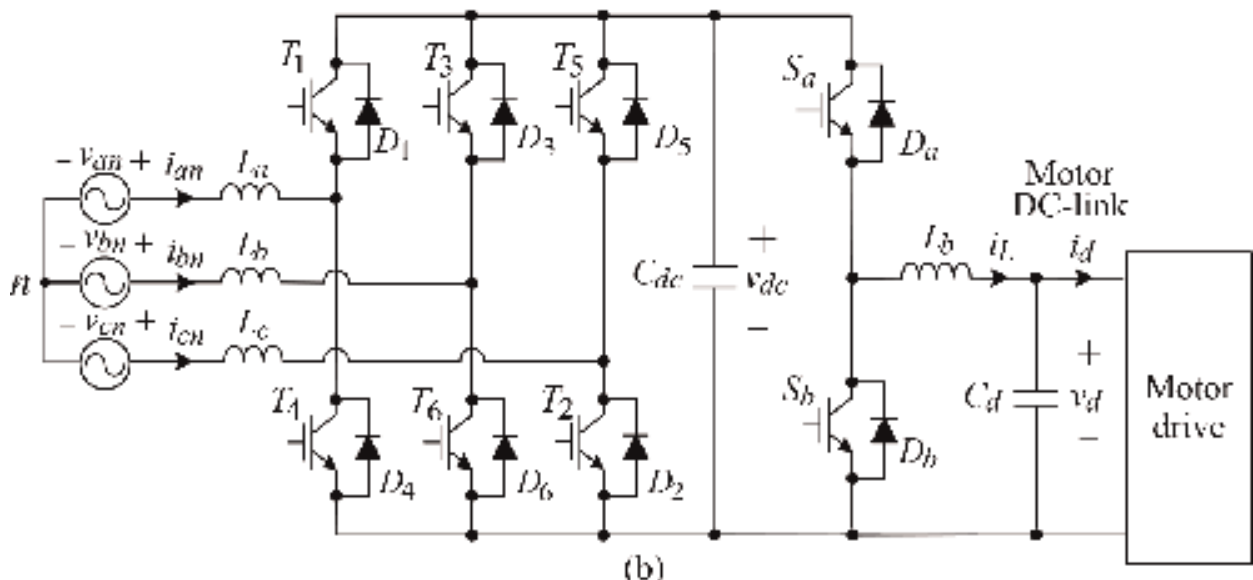

(b)

Figure 12.

Motor drive with bidirectional SMR front end: (a) three-phase SMR and (b) single-phase SMR. 


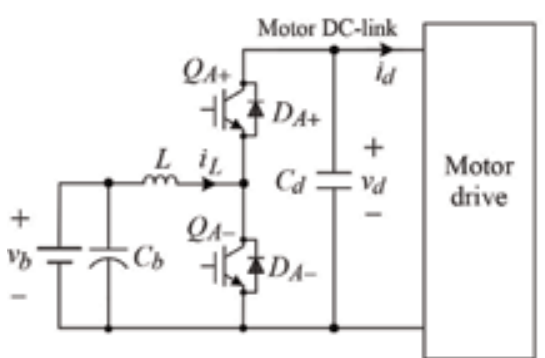

(a)

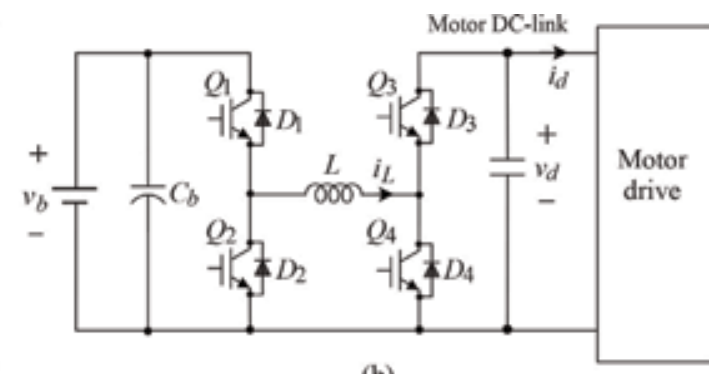

(b)

Figure 13.

Motor drive with bidirectional DC-DC converter front end: (a) one-leg boost-buck converter and (b) H-bridge converter.

F. Generator operation of PMSM: according to Eq. (9), the generating mode of a PMSM is achieved as: PMSG: Q-axis torque current command is directly set to be negative; Regenerative braking and reversible operation of PMSM: the qaxis torque current is automatically set to be negative by the control scheme to achieve normal operations.

G. Sensorless control: basically, the most commonly used position sensorless methods are the observer back-EMF based and the HFI approaches. The former methods can only possess satisfactory running characteristics above a certain speed. Moreover, the motor must be started under traditional synchronous motor mode. By contrast, the HFI approach can be operated effectively in standstill and low speed, so this approach is applicable to frequent-starting occasions, for example, electric vehicles, tractions, elevators, etc. No motor parameters and externally added schematics are needed in constructing a HFI sensorless controlled SynRM drive. However, as mentioned above, the injected signal will yield acoustic noise and lower efficiency, and the back-EMF harmonics of IPMSM and SynRM may cause the estimated rotor position error.

\section{Synchronous reluctance motors}

\subsection{Structural features}

As shown in Figures 3 and 4, SynRM possesses distributed armature windings and PM-free rotor with air slots. The armature is exited with sinusoidal currents. Since it has only the reluctance torque, the developed torque is highly affected by commutation instant setting.

\subsection{Physical modeling}

\subsubsection{Neglecting core loss}

Different from the PMSM shown in Figure 5, the d-axis is conventionally chosen as the reference as indicated in Figure 14a. Following the similar deriving procedure described in the previous section, one can yield the voltage equations of SynRM:

$$
v_{q s}=\left(R_{s}+L_{q} \frac{d}{d t}\right) i_{q s}+\omega_{r} L_{d} i_{d s}, v_{d s}=\left(R_{s}+L_{d} \frac{d}{d t}\right) i_{d s}-\omega_{r} L_{q} i_{q s}
$$




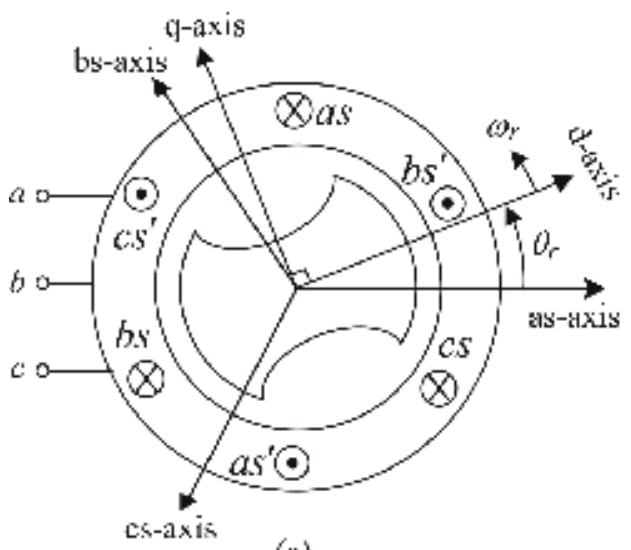

(a)

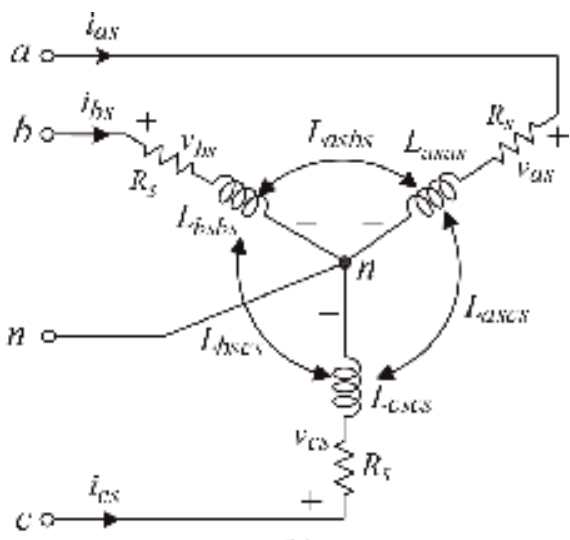

(b)

Figure 14.

A two-pole three-phase synchronous reluctance motor: (a) configuration and (b) Y-connected armature circuit.

$$
L_{q}=L_{l s}+L_{m q}=L_{l s}+\frac{3}{2}\left(L_{A}-L_{B}\right), L_{d}=L_{l s}+L_{m d}=L_{l s}+\frac{3}{2}\left(L_{A}+L_{B}\right)
$$

with $L_{m q}\left(L_{m d}\right)$ being the q-axis (d-axis) magnetizing inductances, respectively. Obviously, $L_{d}>L_{q}$ can be observed from Eq. (16).

The developed torque and mechanical equations of a SynRM drive are:

$$
T_{e}=\frac{3 P}{4}\left(L_{d}-L_{q}\right) i_{q s} i_{d s}=\frac{3 P}{4} \frac{L_{d}-L_{q}}{2} \hat{I}_{a s}^{2} \sin 2 \beta=T_{L}+B \omega_{r}+J \frac{d \omega_{r}}{d t}
$$

where $\hat{I}_{a s}$ is peak of a-phase current, the variable $\beta$ denotes the shift angle between the d-axis and the peak of a-phase current, $i_{q s}=\hat{I}_{a s} \sin \beta$ and $i_{d s}=\hat{I}_{a s} \cos \beta$.

Comments: from Eq. (17) one can find some facts: (i) it only possesses the reluctance torque, which is nonlinear and (ii) although $\beta=45^{\circ}$ can be set to yield the maximum torque, theoretically, the proper setting of $\beta$ is required for maximizing $T_{e}$ in reality.

\subsubsection{Considering core loss}

For a SynRM, the core loss is more significant than other motors. The d-axis and q-axis equivalent circuits considering iron loss with $R_{c}$ are shown in Figure 15a. And Figure 15b shows the phasors of torque currents and terminal currents considering iron loss. From which, the terminal currents $i_{q s}$ and $i_{d s}$ can be expressed using the torque currents $i_{m q}$ and $i_{m d}$ as:

$$
\left[\begin{array}{c}
i_{q s} \\
i_{d s}
\end{array}\right]=\frac{L_{d} L_{q}}{R_{c}}\left[\begin{array}{cc}
\left(s+R_{c} / L_{q}\right) / L_{d} & \omega_{e} / L_{q} \\
-\omega_{e} / L_{d} & \left(s+R_{c} / L_{d}\right) / L_{q}
\end{array}\right]\left[\begin{array}{c}
i_{m q} \\
i_{m d}
\end{array}\right]
$$

And the steady-state expressions of Eq. (4) are:

$$
\left[\begin{array}{c}
I_{q s} \\
I_{d s}
\end{array}\right]=\left[\begin{array}{cc}
1 & L_{d} \omega_{e} / R_{c} \\
-L_{q} \omega_{e} / R_{c} & 1
\end{array}\right]\left[\begin{array}{c}
I_{m q} \\
I_{m d}
\end{array}\right]
$$

The developed torque of a SynRM using $i_{m q}$ and $i_{m d}$ can be expressed as:

$$
T_{e}=\frac{3 P}{4}\left(L_{d}-L_{q}\right) i_{m q} i_{m d}
$$



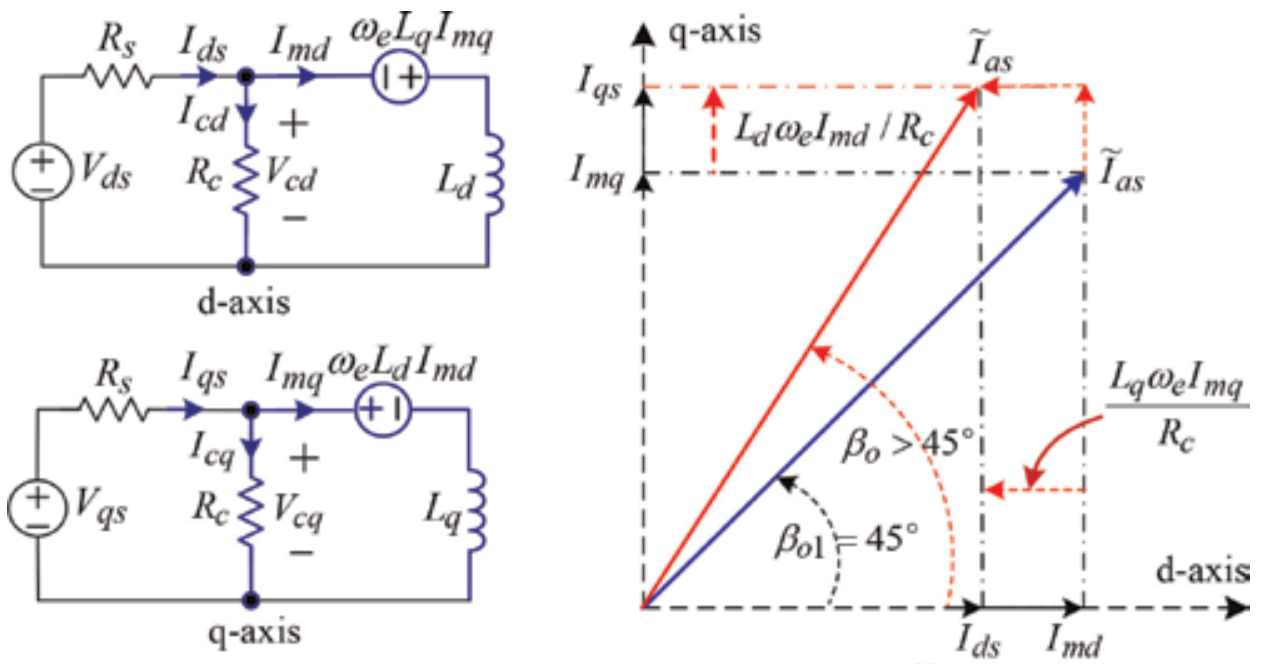

(a)

(b)

Figure 15.

Basic characteristics of SynRM: (a) equivalent circuits considering iron loss and (b) phasors of voltages and currents neglecting core loss.

\subsubsection{Motor losses}

From Figure 15b, the copper loss $P_{c u}$, the core loss $P_{c}$, and the total loss $P_{t}$ of a SynRM can be expressed as:

$$
\begin{gathered}
P_{c u}=\frac{3}{2} R_{s}\left(i_{q s}^{2}+i_{d s}^{2}\right), P_{c}=\frac{3}{2} R_{c}\left(i_{c q}^{2}+i_{c d}^{2}\right) \\
P_{t}=P_{c u}+P_{c}=\frac{3}{2}\left\{\left[R_{s}+\frac{\left(\omega_{e} L_{q}\right)^{2}}{R_{c}}+\frac{\left(\omega_{e} L_{q}\right)^{2} R_{s}}{R_{c}^{2}}\right] i_{m q}^{2}+\left[R_{s}+\frac{\left(\omega_{e} L_{d}\right)^{2}}{R_{c}}+\frac{\left(\omega_{e} L_{d}\right)^{2} R_{s}}{R_{c}^{2}}\right] i_{m d}^{2}\right. \\
\left.+\left[\frac{2 R_{s}}{R_{c}} \omega_{e}\left(L_{d}-L_{q}\right)\right] i_{m q} i_{m d}\right\}
\end{gathered}
$$

By defining the variable $\varsigma=i_{m q} / i_{m d}$, the commutation angle $\beta=\beta_{o}$ is derived to yield the minimum total loss $P_{t}$ listed in Eq. (22):

$$
\partial P_{t} / \partial \varsigma=0, i_{m q} i_{m d}=\text { constant }
$$

Through derivation for Eq. (23) using Eq. (22), one can obtain:

$$
\beta_{o}=\tan ^{-1} \sqrt{\frac{\omega_{e}^{2} L_{d}^{2}\left(R_{s}+R_{c}\right)+R_{s} R_{c}^{2}}{\omega_{e}^{2} L_{q}^{2}\left(R_{s}+R_{c}\right)+R_{s} R_{c}^{2}}}
$$

Comments: (i) from Eq. (24) one can find that by neglecting the core loss $\left(R_{c}=\infty\right)$, the commutation angle will become $\beta=\beta_{o 1}=45^{\circ}$; (ii) normally, $\beta>\left(\beta_{o 1}=45^{\circ}\right)$ for pursuing the optimal efficiencies under varied operating conditions; (iii) as the commutation angle equation of Eq. (24) with nominal parameters of $\left(\bar{L}_{d}, \bar{L}_{q}, \bar{R}_{c}\right)$ is applied, the $\left(\beta=\beta_{o}\right)>45^{\circ}$ is resulted to yield better efficiency. If the accurate fitted parameters $\left(\hat{L}_{d}, \hat{L}_{q}, \hat{R}_{c}\right)$ are available to determine the value of $\left(\beta=\beta_{o}\right)$, a slightly increased efficiency may further be obtained. 


\subsection{Measurement of motor key parameters}

\subsubsection{Winding resistance and inductances}

The estimations of these parameters are similar to those of PMSM described previously.

\subsubsection{Estimation of iron core loss resistance $R_{c}$}

a. As shown in Figure 16, the SynRM is driven under various speeds at no-load with the commutation shift angle of $\beta=45^{\circ}$. The input power $P_{\text {in }}$ and motor line currents are measured. Then the copper loss $P_{c u}$ can be calculated from Eq. (21), and the iron loss $P_{c}$ can be obtained as $P_{c}=P_{\text {in }}-P_{c u}$.

b. The d-axis and q-axis voltages across $R_{c}$ can be found from Figure 15a as:

$$
V_{c d}=V_{d s}-I_{d s} R_{s}, \quad V_{c q}=V_{q s}-I_{q s} R_{s}
$$

c. The equivalent resistance $R_{c}$ can be obtained from Eq. (21) and Figure 15a:

$$
P_{c}=\frac{3}{2} R_{c}\left(\left(\frac{V_{c d}}{R_{c}}\right)^{2}+\left(\frac{V_{c q}}{R_{c}}\right)^{2}\right) \Rightarrow R_{c}=\frac{3}{2} \frac{\left(V_{c d}^{2}+V_{c q}^{2}\right)}{P_{c}}
$$

The estimated $R_{c}$ are $\omega_{r}=500 \mathrm{rpm}, R_{c}=34.71 \Omega ; \omega_{r}=1000 \mathrm{rpm}$, $R_{c}=38.84 \Omega ; \omega_{r}=1500 \mathrm{rpm}, R_{c}=54.34 \Omega ; \omega_{r}=2000 \mathrm{rpm}, R_{c}=64.96$.

\subsubsection{D-axis indexing}

Taking an available three-phase SynRM (4-pole, 550 V, 2000 rpm, 3.7 kW, 17.67 $\mathrm{Nm}$ ) as a test example, which is mechanically coupled with a three-phase PMSM (5 kW, $2000 \mathrm{rpm}, 8$-pole) as its dynamic load.

To verify the correctness of the detected rotor position, the AC voltage is injected into $a$-winding and b-winding terminals as indicated in Figure 14b. Owing to rotor saliency, a-phase current will be an amplitude modulated waveform. Let the PMSM be rotated at $100 \mathrm{rpm}$, the measured a-phase current $i_{a s}$, Hall signal $H_{a}$, and rotor position $\theta_{r}$ due to the excited AC voltage $40 \mathrm{~V} / 60 \mathrm{~Hz}$ which are plotted in

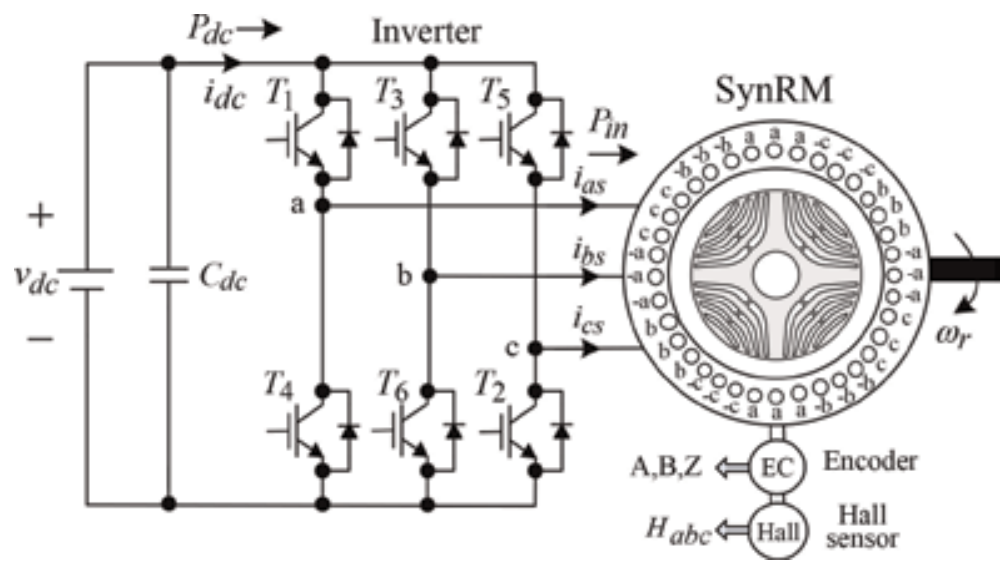

Figure 16.

Core loss resistance estimation mechanism. 


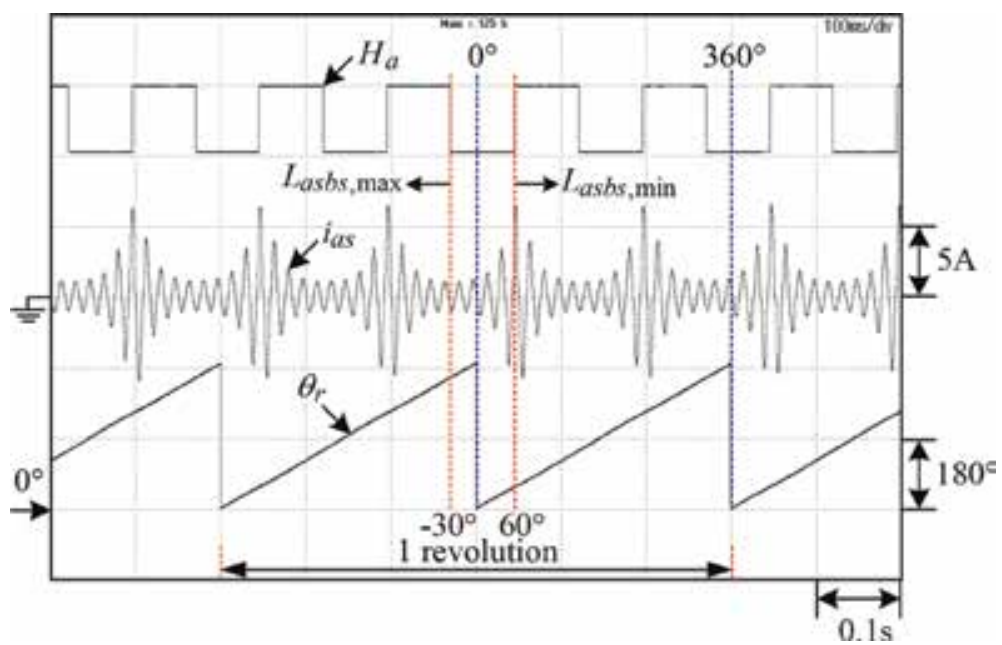

Figure 17.

Measured $\left(H_{a}, i_{a s}, \theta_{r}\right)$ of an example of SynRM under excited AC voltage $40 \mathrm{~V} / 60 \mathrm{~Hz}$.
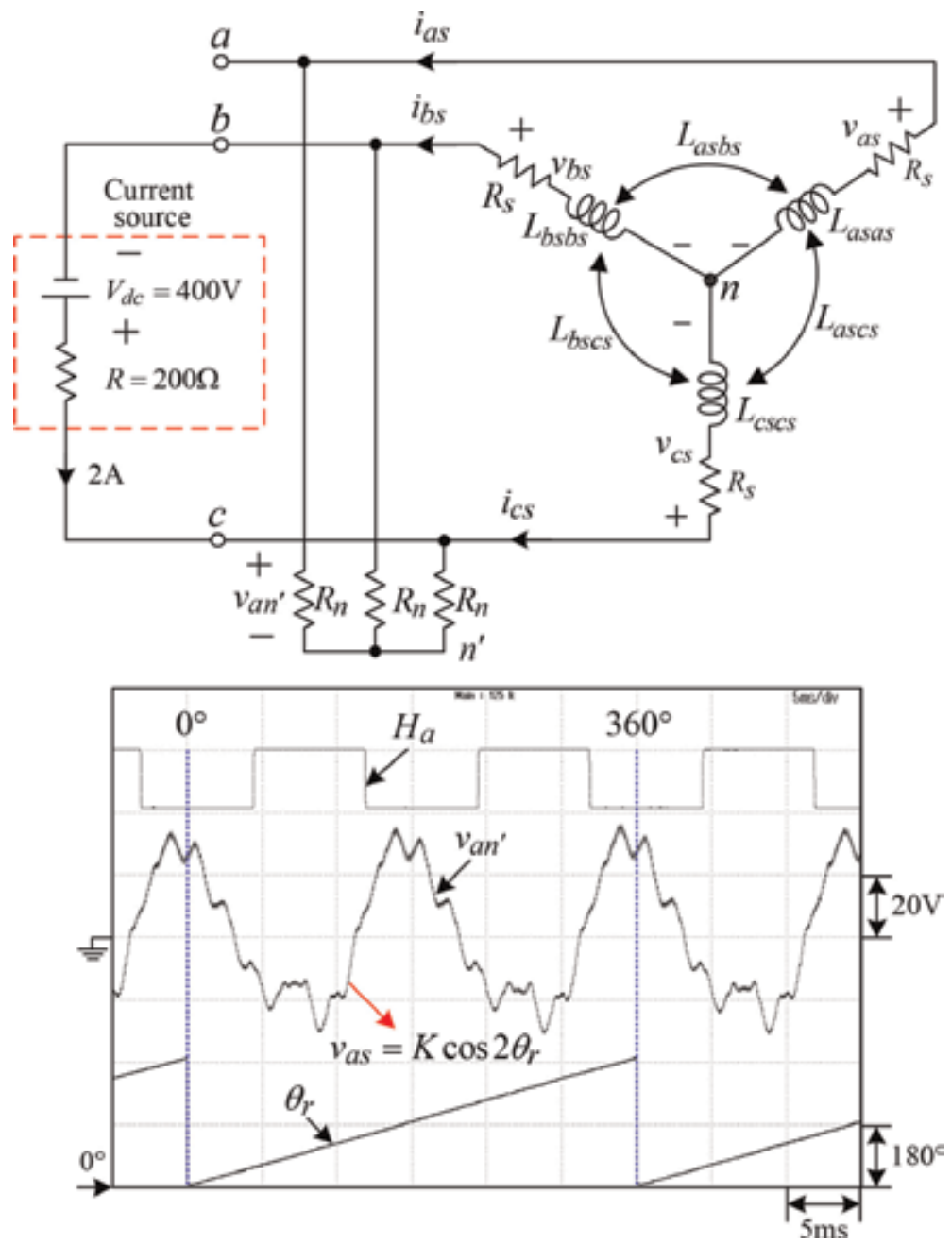

Figure 18.

Configuration of current-injected back-EMF measurement arrangement and the measured Hall signal, back$E M F$, and rotor position. 
Figure 17. It can be expected that the maximum and minimum current $i_{a s}$ occur at $L_{a s b s, \min }$ and $L_{a s b s, \max }$, respectively. From Eq. (10), the $L_{a s b s, \min }$ occurs at $\theta_{r}=\pi / 3$, $L_{a s b s, \max }$ occurs at $\theta_{r}=-\pi / 6$, and the $i_{a s, \max }$ and $i_{a s, \min }$ will also occur at $\theta_{r}=\pi / 3$ and $\theta_{r}=-\pi / 6$, respectively. Hence, from the measured waveforms in Figure 17, one can conclude that the detected rotor position is correct.

\subsubsection{Back-EMF}

From Eqs. (1), (3) and (15), one can find that SynRM possesses no back-EMF under no-load. As for the measurement mechanism proposed in Figure 18, a constant current is injected into the terminals $\mathrm{c}$ and $\mathrm{b}$, and the a-phase terminal voltage $v_{a s} \approx v_{a n}^{\prime}$ is detected and shown in Figure 18, which can be expressed using Eqs. (1) and (3) with $i_{b s}=-i_{c s}=I$ and $i_{a s}=0$ as:

$$
v_{a s}=\frac{d}{d t}\left(\sqrt{3} I L_{B} \sin 2 \theta_{r}\right)=2 \sqrt{3} \omega_{e} I L_{B} \cos 2 \omega_{e} t \underline{\underline{\Delta}} K \cos 2 \theta_{r}, \quad \theta_{r}=\omega_{e} t
$$

From the measured waveforms shown in Figure 18, one can be aware of some facts: (i) the adequacy of position sensing can be observed and (ii) the slotting effects can be comprehended from the ripples contaminated on $v_{a n^{\prime}}$.

\subsection{Performance test of motor drive}

Figure 19a and $\mathbf{b}$ shows the motor drive running characteristic test environment using eddy current brake and the suggested alternative test environment using load SPMSG as a dynamic load. Since the accurate eddy current brake and torque meter are not available, this alternative of loading test is worthy of adopting. However, the surface-mounted permanent-magnet synchronous generator (SPMSG) must be properly set, and it should be noted that the motor efficiency is both speed and load dependent.

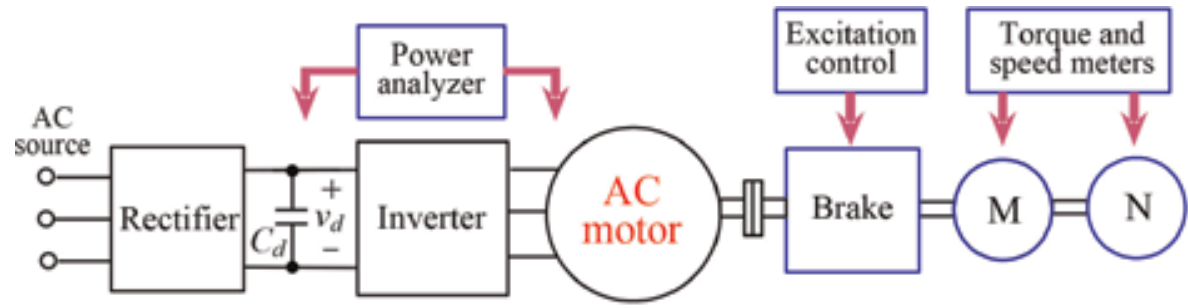

(a)

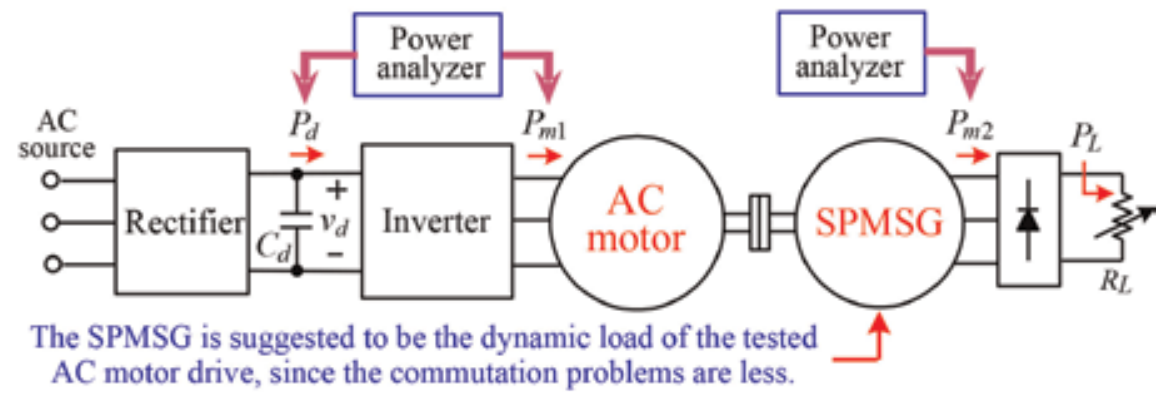

(b)

Figure 19.

Test facilities for AC motor drive: (a) running characteristic test using eddy current brake and $(b)$ running characteristic test using load SPMSG as dynamic load. 


\section{A battery/supercapacitor-powered EV PMSM drive}

\subsection{System configuration}

Figure 20a shows the developed battery/super-capacitor (SC)-powered electric vehicle (EV) IPMSM drive with proper interface power converters. The control scheme motor drive is shown in Figure 20b, whereas the control schemes of other power stages are neglected here.

\subsubsection{IPMSM drive}

\subsubsection{System components}

The used IPMSM is rated as three-phase 6-pole, $3000 \mathrm{rpm}, 1 \mathrm{~kW}, 4.8 \mathrm{~A}$, and $3.23 \mathrm{Nm}$. A surface-mounted PMSG is used as the dynamic load. The equipped dynamic brake leg is formed by $\left(T_{B 1}, R_{B}, D_{B 1}\right)$ with the brake resistor $R_{B}=25 \Omega / 200 \mathrm{~W}$.

\subsubsection{Operation control}

a. Motoring mode: the positive torque current command $i_{q s}^{*}$ yielded from the outer speed loop together with the properly set field excitation current command $i_{d s}^{*}$ let the IPMSM to yield positive developed torque for driving control. The improved generated torque can be obtained by commutation advanced shift and voltage boosting. The d-axis current command is set to yield the maximum developed torque within rated speed:

$$
i_{d s}^{*}=\frac{\lambda_{m}^{\prime}}{2\left(L_{q}-L_{d}\right)}-\sqrt{\frac{\left(\lambda_{m}^{\prime}\right)^{2}}{4\left(L_{q}-L_{d}\right)^{2}}+\left(i_{q s}^{*}\right)^{2}}
$$

b. Regenerative braking: as the speed command is decreased, the negative speed tracking error $\varepsilon_{\omega}$ will let the torque current command $i_{q s}^{*}$ become negative automatically. The recovered motor power will be sent back to charge the battery.

\subsubsection{Battery interface converter}

\subsubsection{System components}

The battery bank is formed by two serially connected cells (UC BATTERY PS 40138, $72 \mathrm{~V} 30 \mathrm{Ah}$ ) with nominal voltage $V_{b}=156 \mathrm{~V}$. The DC-link voltage is arranged as $V_{d c, \min }=100 \mathrm{~V}$ (buck mode), $V_{d c, \max }=400 \mathrm{~V}$ (boost mode).

\subsubsection{Operation control}

\subsection{Discharging mode}

As shown in Figure 20a, through the H-bridge DC/DC converter, the battery $\left(v_{b}=156 \mathrm{~V}\right)$ establishes the adjustable and well-regulated DC-link voltage 


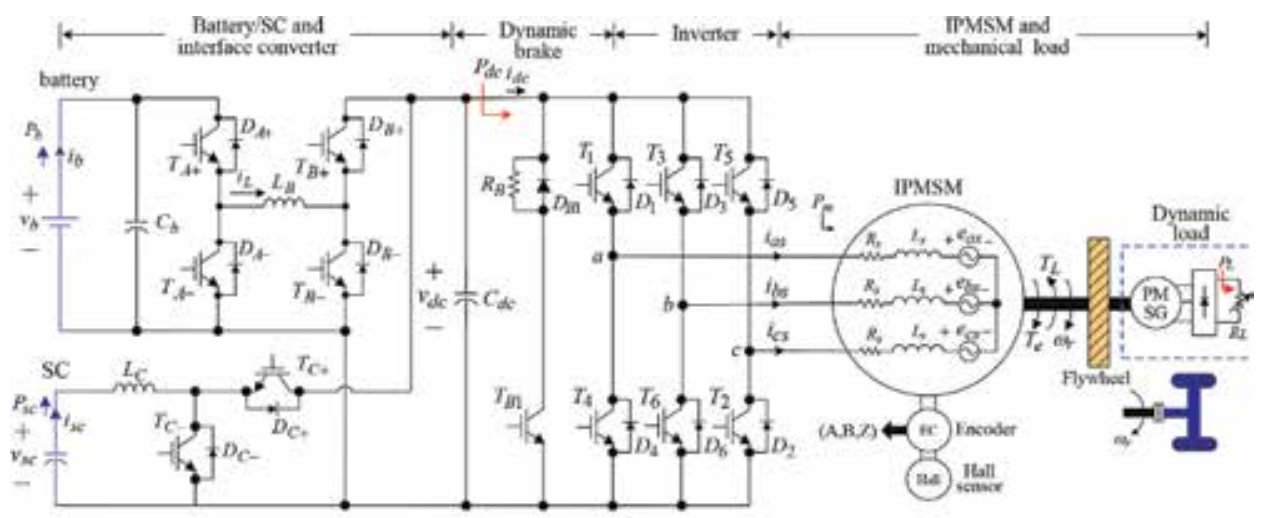

(a)

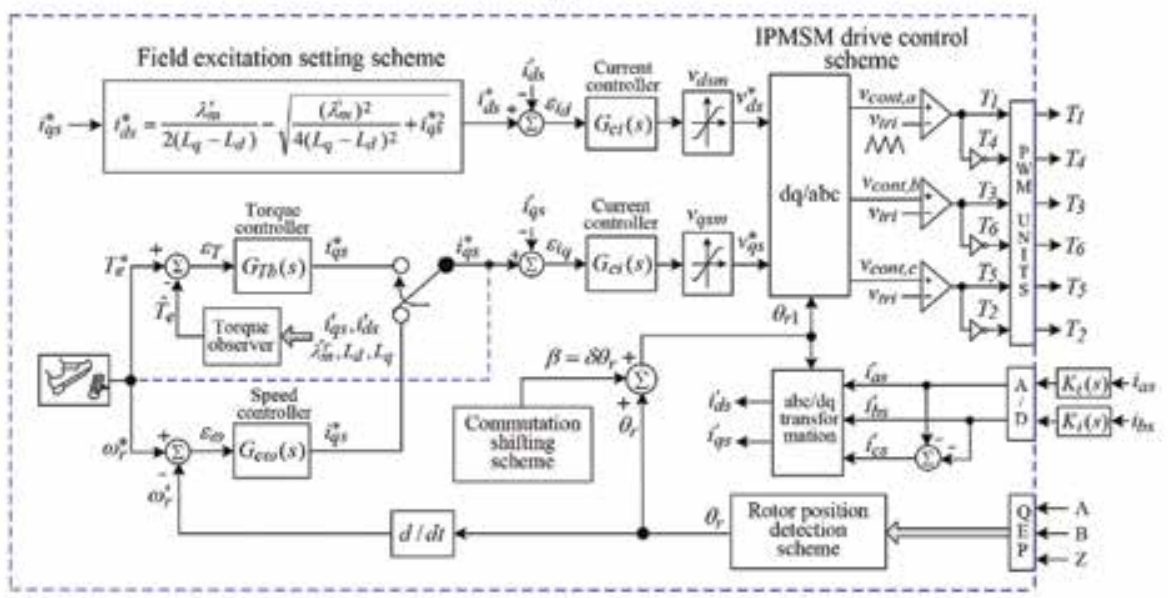

(b)

Figure 20.

The developed EV IPMSM drive: (a) schematic and (b) IPMSM drive control scheme.

$\left(v_{d c}<v_{b}\right.$ or $\left.v_{d c} \geq v_{b}\right)$. Here, the lowest and highest voltages are set as $100 \mathrm{~V}$ and $400 \mathrm{~V}$, respectively. The buck converter is formed by $\left(T_{A+}, D_{A-}, L_{B}, D_{B+}\right)$, while the boost converter is constructed using $\left(T_{A+}, T_{B+}, D_{B-}, L_{B}\right)$.

\subsection{Charging mode}

In making battery charging during motor regenerative braking, the DC-link voltage is regulated at the set value, and the front-end converter is operated in reverse direction automatically. The components $\left(T_{B-}, D_{B+}, L_{B}, D_{A+}\right)$ and $\left(T_{A-}, D_{A+}, L_{B}\right.$, $T_{B+}$ ) form the buck and boost converters, respectively. The maximum battery charging current is equivalently set by $i_{L m}^{*}=12 \mathrm{~A}$ of the current limiter.

\subsubsection{SC interface converter}

\subsubsection{System components}

The SC bank is rated as 6F/160 V, BMOD0006 E160 B02 (Maxwell Technologies), and maximum current $=170 \mathrm{~V}$, maximum continuous current $=7 \mathrm{~A}$, ESR $(\mathrm{DC})=240 \mathrm{~m} \Omega$. The system voltage and power ratings are set as $V_{s c}=100 \mathrm{~V}$, $V_{d c}=400 \mathrm{~V}$, and $P_{d c}=1 \mathrm{~kW}$. 


\subsubsection{Operation control}

\subsection{Motoring mode}

In motoring mode, the DC-link voltage command $v_{d c}^{*}$ of the SC is set slightly higher $(405 \mathrm{~V})$ than the nominal DC-link voltage $V_{d c}(400 \mathrm{~V})$. This will allow the switch $T_{C-}$ be operated to discharge the SC stored energy for acceleration. Then the battery is discharged subsequently as the energy of SC is exhausted.

\subsection{Regenerative braking mode}

As the braking is commanded, the DC-link voltage will be larger than its command due to the recovered motor stored kinetic energy. Hence the negative value of voltage tracking error will let the current command of current loop in the SC energy storage system become negative. The switch $T_{C+}$ will be operated to recycle the energy into the SC. Similarly, the maximum SC charging current is set by $20 \mathrm{~A}$ of the current limiter.

\subsubsection{Adjustable DC-link voltage}

For an inverter-fed motor drive, the lower DC-link voltage under lower speed and lighter load may yield smaller switching losses. Moreover, the EV is not often operated at relatively higher speed. As a result, the DC-link voltage can be properly set according to the motor running velocity to obtain higher efficiency over wide speed range. Most existing EV PCUs can only provide boosted motor drive DC-link voltage from battery. For the developed EV IPMSM drive, thanks to the flexibility possessed by the H-bridge DC/DC converter, the DC-link voltage can be lower than the battery voltage. The proposed DC-link voltage versus speed profile is depicted in Figure 21. The DC-link voltage profiles set for comparison are:

1. Fixed DC-link voltage: $v_{d c}=v_{d c 1}=400 \mathrm{~V}, 0 \leq \omega_{r} \leq 4600 \mathrm{rpm}\left(0 \leq \omega_{r} \leq \omega_{m}\right)$

2. Varied DC-link voltage:

a. $v_{d c}=v_{d c 2}=100 \mathrm{~V}, 0 \leq \omega_{r} \leq 1000 \mathrm{rpm}\left(0 \leq \omega_{r} \leq \omega_{1}\right)$.

b. $100 \mathrm{~V}<\left(v_{d c}=v_{d c 2}\right) \leq 400 \mathrm{~V}, 1000 \mathrm{rpm} \leq \omega_{r} \leq 4600 \mathrm{rpm}$ $\left(\omega_{1}<\omega_{r} \leq \omega_{m}\right)$

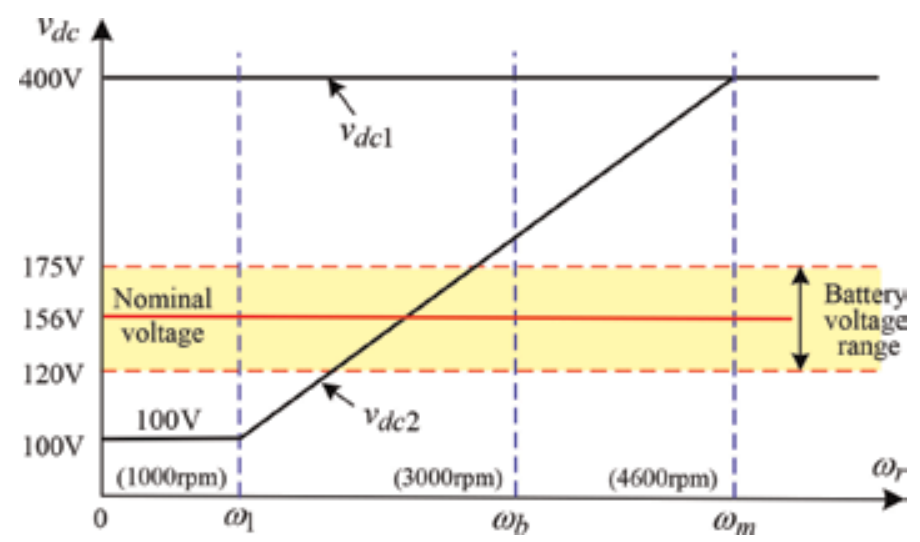

Figure 21.

The proposed DC-link voltage profile. 


\subsection{Some experimental results}

\subsubsection{Motor drive without SC}

\subsubsection{Steady-state characteristics}

By setting $i_{d s}^{*}$ according to Eq. (26), Figure 22 shows the measured $H_{a}$ and $\left(i_{a s}^{*}, i_{a s}, i_{d s}^{*}, i_{d s}^{\prime}\right)$ at $\left(v_{b}=156 \mathrm{~V}, v_{d c}=400 \mathrm{~V}, R_{L}=44.7 \Omega, \omega_{r}^{*}=3000 \mathrm{rpm}\right)$ of the developed EV IPMSM drive powered by battery H-bridge bidirectional DC/DC converter. The results show that the phase winding current commands $i_{a s}^{*}$ are properly shifted with respect to the sensed Hall signal $H_{a}$, and the $i_{a s}^{*}$ closely follows its command.

\subsubsection{Acceleration/deceleration/reversible and regeneration braking operation}

Figure 23 shows the measured $\left(\omega_{r}^{*}, \omega_{r}^{\prime}\right)$ and $\left(i_{q s}^{*}, i_{q s}^{\prime}\right)$ at $\left(v_{b}=156 \mathrm{~V}, v_{d c}\right.$ $=400 \mathrm{~V}, R_{L}=100.7 \Omega$ ) of the EV IPMSM drive powered by H-bridge DC/DC frontend converter under forward and reversible operations, with the speed command being set as $(3000 \mathrm{rpm})$ to $(-3000 \mathrm{rpm})$ and the rate of $600 \mathrm{rpm} / \mathrm{s}$. In order to let the IPMSM drive be reversibly operated, the torque current component is made opposite automatically, which can be seen from the results. The smooth speed forward and reversible operations can be seen from the results.

\subsubsection{Comparative evaluation for fixed and varied-voltage DC-links}

The measured $\left(v_{d c}^{*}, v_{d c}^{\prime}, \omega_{r}^{*}, \omega_{r}^{\prime}, v_{b}, i_{b}, P_{b}, E_{b}\right)$ of the EV IPMSM drive powered by $\mathrm{H}$-bridge DC/DC converter at $\left(R_{L}=75 \Omega\right)$ under the speed pattern defined by the ECE15. The speed pattern defined by ECE15 with fixed $v_{d c}=400 \mathrm{~V}$ and varied $v_{d c}$ are compared in Figure 24a and $\mathbf{b}$, where $P_{b} \equiv v_{b} \times i_{b}$ and $E_{b} \equiv \int P_{b} d t$. The developed EV IPMSM drives with fixed and varied $v_{d c}$ all yield satisfactory driving characteristic.

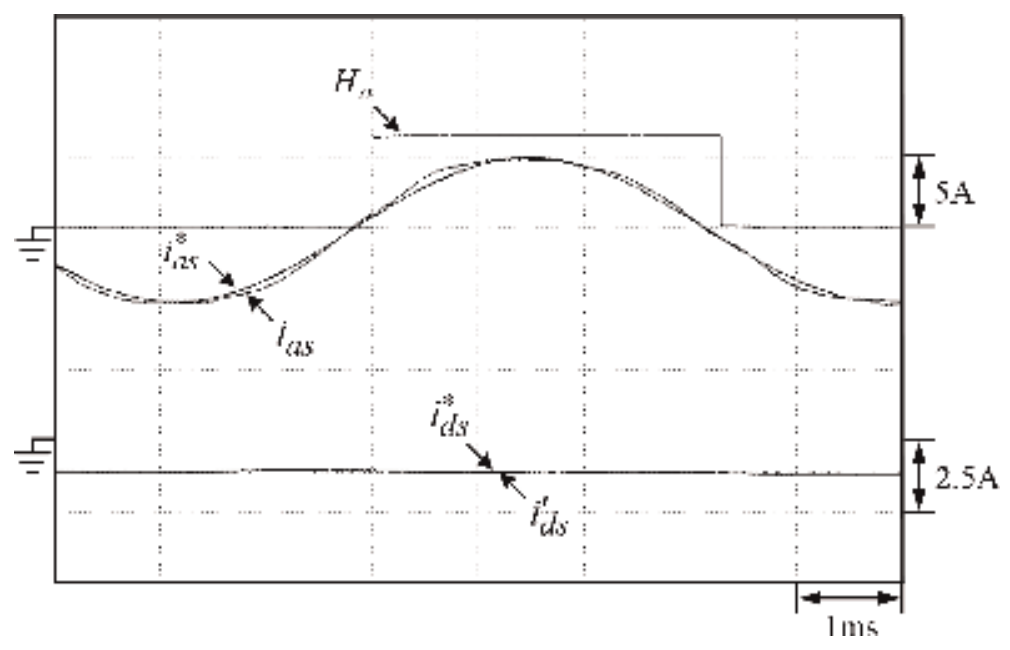

Figure 22.

Measured $H_{a}$ and $\left(i_{a s}^{*}, i_{a s}, i_{d s}^{*}, i_{d s}^{\prime}\right)$ of the developed standard IPMSM drive powered by H-bridge DC/DCfrontend converter at $\left(v_{b}=156 \mathrm{~V}, \omega_{r}^{*}=3000 \mathrm{rpm}, R_{L}=44.7 \Omega\right)$ with $v_{d c}=400 \mathrm{~V}$ and $i_{d s}^{*}=-1 \mathrm{~A}$ being set $\left(\hat{i}_{a s}=5.15 A\right)$. 


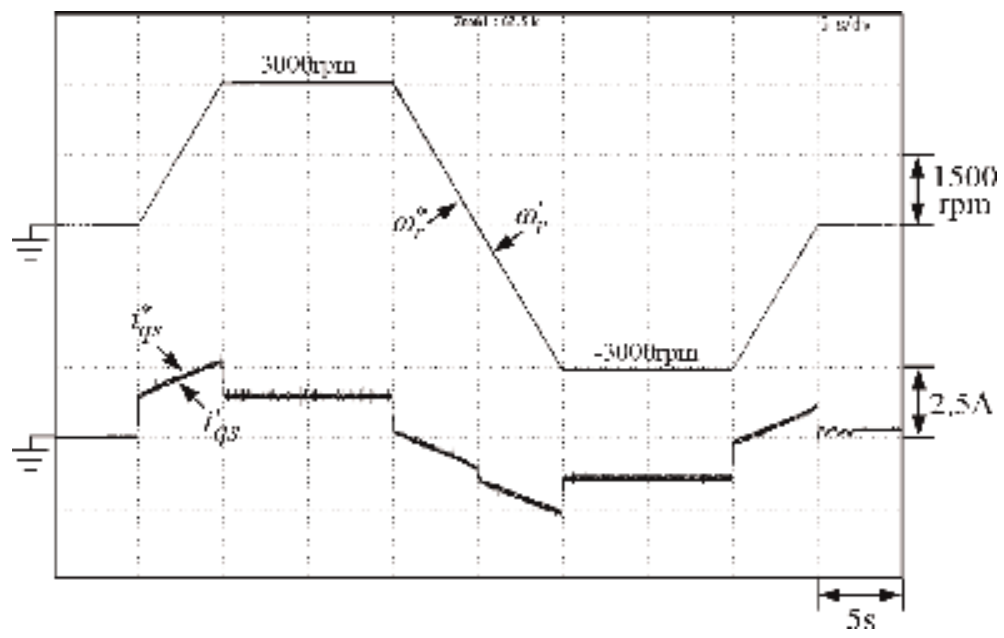

Figure 23.

Measured $\left(\omega_{r}^{*}, \omega_{r}^{\prime}, i_{q s}^{*}, i_{q s}^{\prime}\right)$ of the developed IPMSM drive powered by $H$-bridge DC/DC front-end converter due to speed command change of $\left(\omega_{r}=0 \mathrm{rpm} \rightarrow 3000 \mathrm{rpm} \rightarrow 0 \mathrm{rpm} \rightarrow-3000 \mathrm{rpm} \rightarrow 0 \mathrm{rpm}\right)$ under $\left(v_{b}=156 \mathrm{~V}, v_{d c}=400 \mathrm{~V}, R_{L}=100.7 \Omega\right)$.

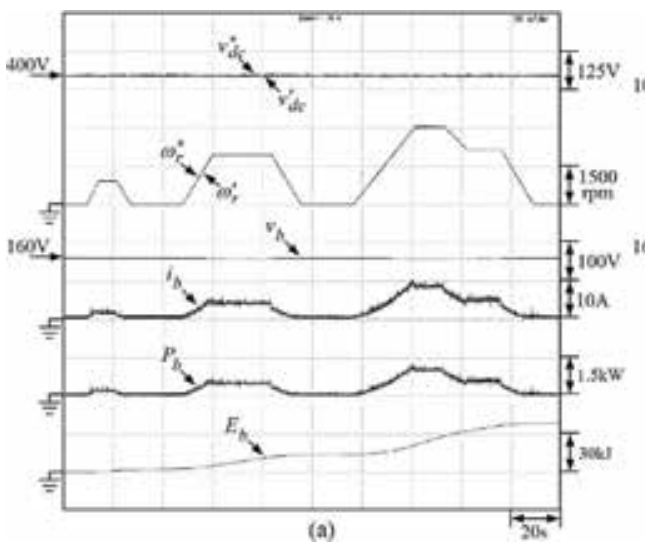

(a)

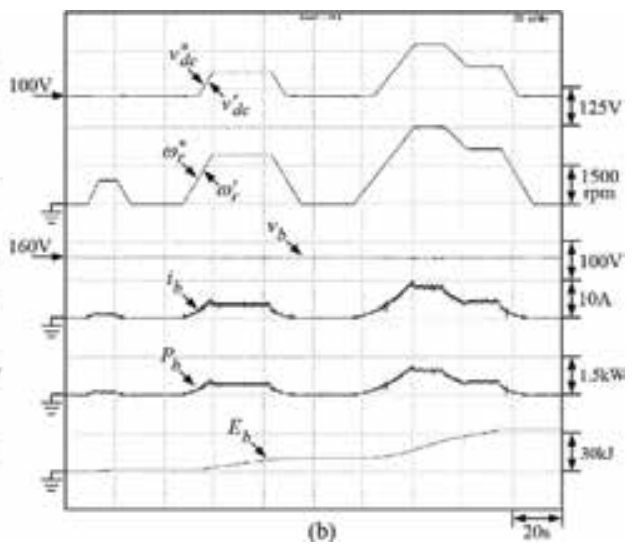

(b)

Figure 24.

Measured $\left(v_{d c}^{*}, v_{d c}^{\prime}, \omega_{r}^{*}, \omega_{r}^{\prime}, v_{b}, i_{b}, P_{b}, E_{b}\right)$ of the developed EV IPMSM drive powered by H-bridge DC/DC front-end converter at $\left(R_{L}=75 \Omega\right)$ due to the speed pattern defined by the ECE15: (a) $v_{d c}$ is fixed at $400 \mathrm{~V}$ and $(b) v_{d c}$ is varied with speed.

The measured $E_{b}$ by the fixed $v_{d c}$ and the varied $v_{d c}$ corresponding to Figure 24 are further compared in Figure 25. From the comparison one can find that the adjustable DC-link voltage can reduce battery energy consumption.

\subsubsection{Motor drive incorporated with SC}

Under the preset speed changing pattern as shown in Figure 26, the measured results of the established EV IPMSM drive powered by H-bridge DC/DC front-end converter with acceleration and deceleration rates of $750 \mathrm{rpm} / \mathrm{s}$ at $\left(v_{b}=156 \mathrm{~V}, v_{d c}^{*}=400 \mathrm{~V}, v_{s c}^{*}=405 \mathrm{~V}, R_{L}=75 \Omega\right)$ are provided as: Figure 26a: $\left(\omega_{r}^{*}, \omega_{r}^{\prime}, i_{b}, v_{b}, v_{d c}\right)$ without SC; Figure 26b: $\left(\omega_{r}^{*}, \omega_{r}^{\prime}, i_{b}, i_{s c}, v_{s c}, v_{b}, v_{d c}\right)$ with SC. Without SC, the battery possesses larger charging and discharging currents as shown in Figure 26a.

The application of SC can reduce the battery currents in driving and regenerative braking operations under the same scenario. In the results presented in Figure 26b with SC using active discharging approach (noted that $v_{s c}^{*}>v_{d c}^{*}$ ), the battery current remains zero at the beginning under driving mode, the SC 


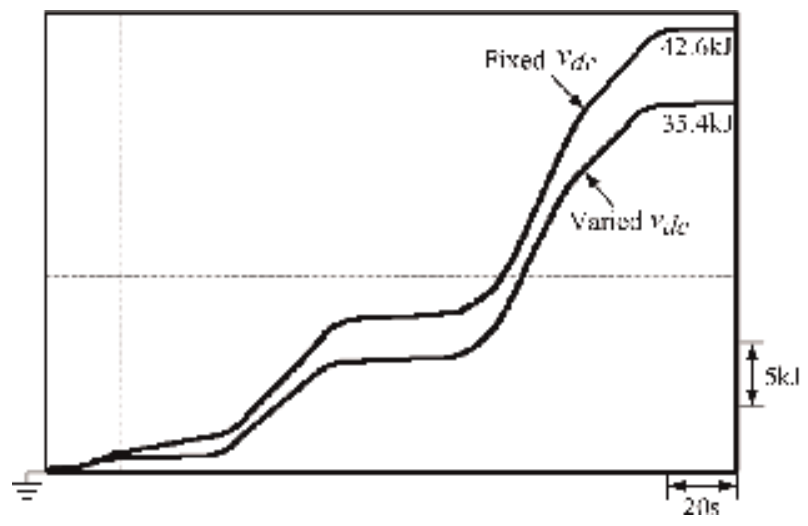

Figure 25 .

Measured $E_{b}$ of the developed standard IPMSM drive powered by H-bridge converter with fixed $v_{d c}=400 \mathrm{~V}$ and varied $v_{d c}$ due to speed pattern defined by the ECE15 at $\left(R_{L}=75 \Omega\right)$.

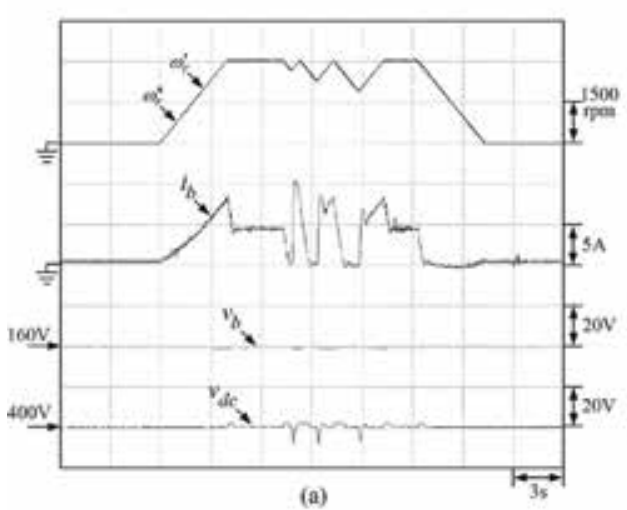

(a)

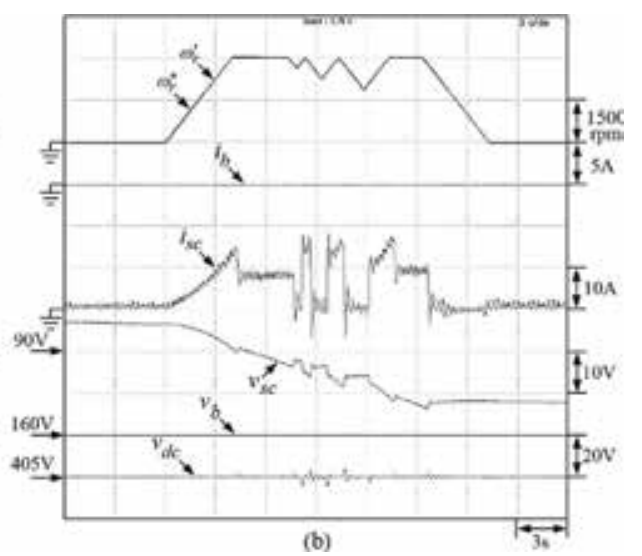

(b)

Figure 26.

Measured results of the established standard EV IPMSM drive powered by H-bridge DC/DC front-end converter due to preset speed pattern with acceleration and deceleration rates of $750 \mathrm{rpm} / \mathrm{s}$ at $\left(v_{b}=156 \mathrm{~V}, v_{d c}^{*}=400 \mathrm{~V}, v_{s c}^{*}=405 \mathrm{~V}, R_{L}=75 \Omega\right):(a)\left(\left(\omega_{r}^{*}, \omega_{r}^{\prime}\right), i_{b}, v_{b}, v_{d c}\right)$ without SC and (b) $\left(\left(\omega_{r}^{*}, \omega_{r}^{\prime}\right), i_{b}, i_{s c}, v_{s c}, v_{b}, v_{d c}\right)$ with $S C$ under discharging operation.

discharges via its bidirectional converter first, and the battery pack does not supply power to DC link until the SC is unable to maintain the driving torque. Hence, not only the energy of regenerative braking can be recycled, but also the battery can avoid its intermittent charging/discharging operations. Obviously, by adding the supercapacitor storage system, the battery provides the fewer power during acceleration condition, and its current would never become negative. Besides, the battery voltage is also less fluctuated.

\section{A bidirectional SMR-fed synchronous reluctance motor drive}

\subsection{System configuration}

Figure 27a shows the developed SMR-fed SynRM drive. And the control scheme of the motor drive is depicted in Figure $27 \mathbf{b}$. The three-phase full-bridge SMR is employed to power the motor drive from the mains with good line drawn power quality. The boosted and well-regulated DC-link voltage enhances motor driving performance over wide speed range. Moreover, the recovered energy during regenerative braking can be successfully sent back to utility grid. 

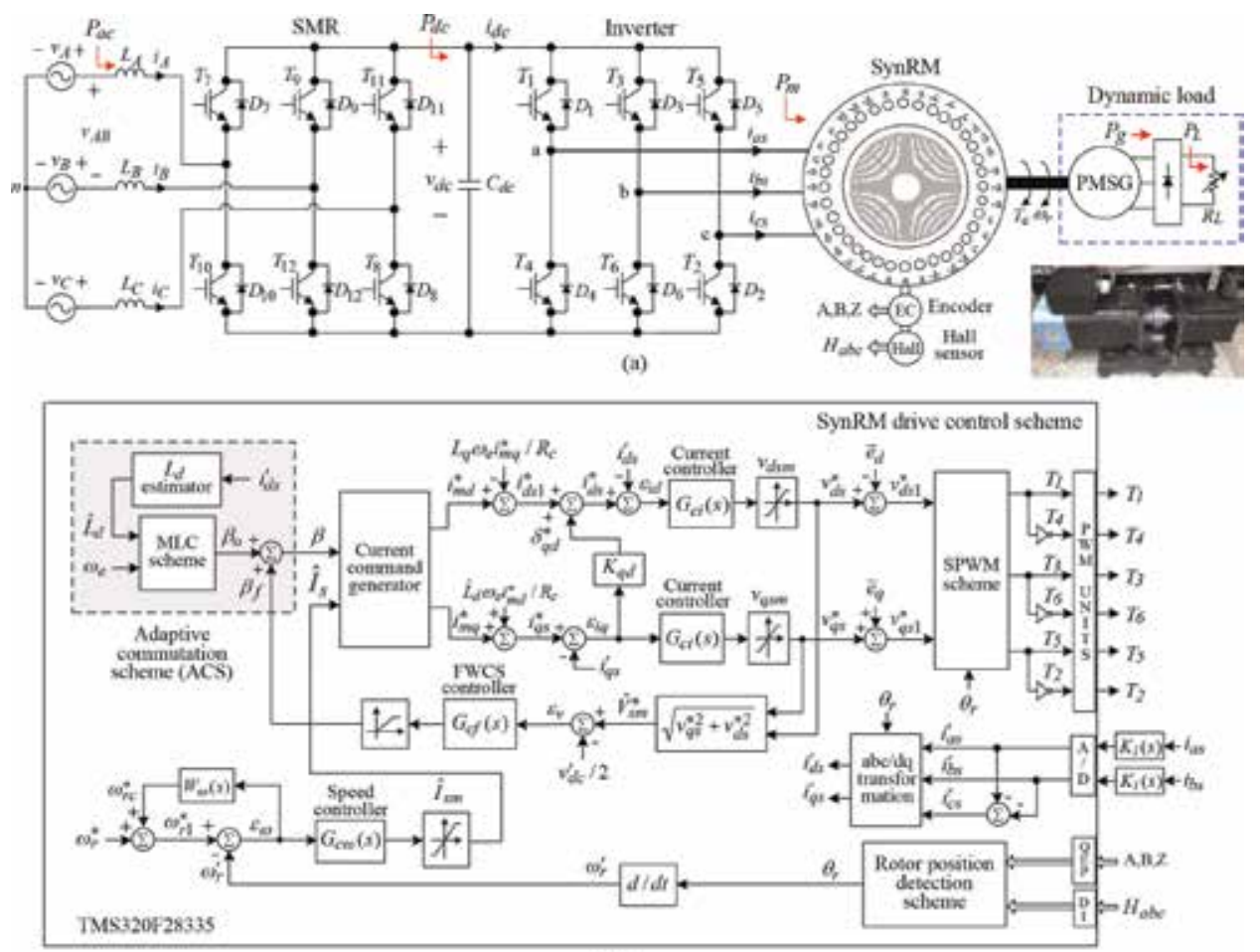

(b)

Figure 27.

The SMR-fed SynRM drive: (a) schematic and (b) motor drive control scheme.

In treating the driving control for a SynRM drive, some distinct features are worth noting: (i) since only reluctance torque is exited, the proper commutation angle setting is the most critical issue affecting its developed torque characteristics; (ii) the iron loss of SynRM is significant and varied with operating conditions; and (iii) the d-axis inductance is significantly changed with the armature current level due to magnetic saturation; hence, the iron loss and copper loss should be simultaneously considered to yield the improved overall efficiency. In the SynRM drive control scheme shown in Figure $\mathbf{2 7} \mathbf{b}$, the proposed improved approaches include (i) the commutation angle is set by an adaptive commutation scheme (ACS) using online estimated d-axis inductance to minimize motor total loss. Under higher speed, an extra commutation angle is yielded by the field-weakening commutation scheme (FWCS) to limit the voltage. And (ii) in the proposed ramp-comparison current-controlled PWM (RC-CCPWM) scheme, the basic feedback control is augmented with a back-EMF elimination feedforward controller and a transient crosscoupling field controller to enhance the current control performance.

\subsubsection{SynRM drive}

\subsubsection{System components}

a. The employed SynRM is rated as SynRM: 4-pole, $550 \mathrm{~V}, 2000 \mathrm{rpm}, 3.7 \mathrm{~kW}$. The inverter is formed using three Mitsubishi IGBT modules CM100DY-12H $\left(V_{C E}=600 \mathrm{~V}, I_{C}=100 \mathrm{~A}, I_{C M}=200 \mathrm{~A}\right)$. The SPWM switching is employed. A PMSG with load resistance $R_{L}$ is served as the dynamic load of the studied SynRM. 
b. Estimated motor parameters: $R_{s}=0.47 \Omega, R_{c}=61.37 \Omega, L_{q}=28.92 \mathrm{mH}$ (at $66.6 \mathrm{~Hz}$ ). The d-axis inductance $L_{d}$ is much affected by the magnetic saturation compared to the q-axis inductance $L_{q}$. The fitted $\hat{L}_{d}\left(i_{m d}\right)($ in $\mathrm{H})$ is expressed as:

$$
\hat{L}_{d}\left(i_{m d}\right)=\left\{\begin{array}{c}
0.0559(\mathrm{H}), i_{m d} \leq 3 \mathrm{~A} \\
\alpha_{1} \ln \left(i_{m d}\right)+\alpha_{2}(\mathrm{H}), i_{m d}>3 \mathrm{~A}
\end{array}\right.
$$

where $\alpha_{1}=-0.00864$ and $\alpha_{2}=-0.06458$.

\subsubsection{Commutation scheme}

a. The proposed adaptive commutation scheme (ACS): the fitted $\hat{L}_{d}\left(i_{m d}\right)$ and the estimated motor parameters are applied to Eq. (24) to determine the commutation angle $\left(\beta=\beta_{o}\right)$.

b. Field-weakening commutation scheme (FWCS)

Under higher speeds, the field weakening via commutation advanced shift must be applied to satisfy the electrical capabilities of a SynRM drive expressed as:

$$
\begin{gathered}
\hat{I}_{s}=\sqrt{i_{q s}^{2}+i_{d s}^{2}} \leq \hat{I}_{s m} \\
\hat{V}_{s}=\sqrt{v_{q s}^{2}+v_{d s}^{2}} \leq \hat{V}_{s m}=v_{d c} / 2
\end{gathered}
$$

where $\hat{I}_{s}\left(\hat{V}_{s}\right)=$ phase current (voltage) magnitude and the maximum voltage $\hat{V}_{s m}=v_{d c} / 2$ is set for a SPWM inverter.

For regulating the voltage limit automatically, the commutation shift angle $\beta_{f}$ is yielded by the proposed FWCS scheme shown in Figure $27 \mathbf{b}$ as:

$$
\beta_{f}=\left\{\begin{array}{cc}
0, & \sqrt{v_{q s}^{* 2}+v_{d s}^{* 2}}<\left(\frac{v_{d c}^{\prime}}{2}\right) \\
G_{c f}(s)\left(\sqrt{v_{q s}^{* 2}+v_{d s}^{* 2}}-\left(\frac{v_{d c}^{\prime}}{2}\right)\right), \sqrt{v_{q s}^{* 2}+v_{d s}^{* 2}} \geq\left(\frac{v_{d c}^{\prime}}{2}\right)
\end{array}\right.
$$

\subsubsection{Three-phase full-bridge SMR}

To simultaneously preserve bidirectional power flow and power factor corrected capabilities, the three-phase full-bridge SMR is established and employed as the AC front end of the SynRM drive. The three-phase AC input line voltage is $220 \mathrm{~V} /$ $60 \mathrm{~Hz}$. And the DC-link voltage is set as $V_{d c}=550 \mathrm{~V}$. Three IGBT modules CM100DY-12H are used to form the power circuit.

\subsection{Some experimental results}

\subsubsection{Performance of SMR front end}

Figure 28a and $\mathbf{b}$ shows the steady-state characteristics of the three-phase fullbridge SMR-fed SynRM drive at $\left(V_{d c}=550 \mathrm{~V}, \omega_{r}=2000 \mathrm{rpm}, \beta=\beta_{o 1}=45^{\circ}\right.$, $R_{L}=13.7 \Omega$ ). The results indicate that the SMR possesses good operating 


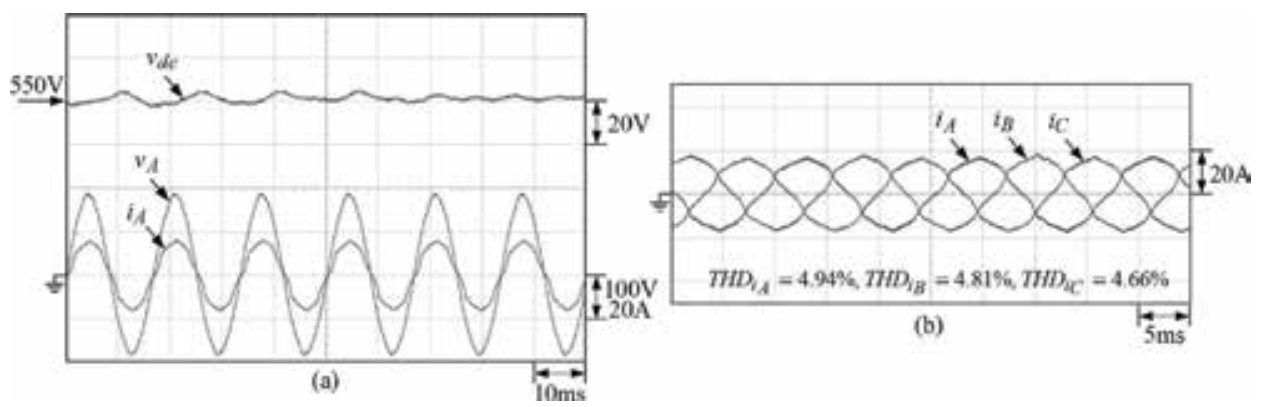

Figure 28.

Measured steady-state results of the developed SMR-fed SynRM drive under $\left(V_{d c}=550 \mathrm{~V}, \omega_{r}^{\prime}=2000 \mathrm{rpm}\right.$, $\left.\beta=45^{\circ}, R_{L}=13.7 \Omega\right):(a)\left(v_{d c}, v_{A}, i_{A}\right)$ and $(b)\left(i_{A}, i_{B}, i_{C}\right)$.

characteristics in powering the followed SynRM drive, good line drawn power quality, and well-regulated DC-link voltage which are seen.

\subsubsection{Effectiveness of the proposed ACS scheme}

Under $\left(V_{d c}=550 \mathrm{~V}, R_{L}=13.7 \Omega\right)$, let the commutation angles be $\beta=\beta_{o 1}=45^{\circ}$ and $\beta=\beta_{o}$ yielded from Eq. (24); the measured $\left(\omega_{r}^{\prime}, \hat{I}_{s}, P_{d c}\right)$ of the SynRM drive at two speeds are compared in Figure 29a and $\mathbf{b}$. One can find that the proposed commutation angle setting can lead to less power consumption and the reduction of current magnitude.

\subsubsection{Effectiveness of the proposed FWCS in high-speed driving performance}

Since the rated speed of the used SynRM is $2000 \mathrm{rpm}$, the proper field weakening is necessary under the speeds larger than $2000 \mathrm{rpm}$. Let the commutation angle be $\beta=\beta_{o 1}+\beta_{f}$ with $\beta_{o 1}=45^{\circ}$ being set and $\beta_{f}$ being yielded by the developed FWCS. Figure 30 plots the measured $\left(\omega_{r}^{*}, \omega^{\prime}{ }_{r}\right)$ and the yielded commutation angle $\beta_{f}$ during starting process at $\left(V_{d c}=550 \mathrm{~V}, R_{L}=303.9 \Omega\right)$ from 0 to $4000 \mathrm{rpm}$ with an acceleration rate of $(250 \mathrm{rpm} / \mathrm{s})$. One can find from the results that speed tracking error under high speeds can be eliminated by applying the FWCS via increasing the commutation angle component $\beta_{f}$.

\subsubsection{Reversible operation}

The measured $\left(\omega_{r}^{*}, \omega_{r}^{\prime}\right)$ and $\hat{I}_{s}$ of the SMR-fed SynRM drive at $\left(V_{d c}=550 \mathrm{~V}, R_{L}=13.7 \Omega\right)$ with the commutation angle $\beta=\beta_{o}$ due to the ramp
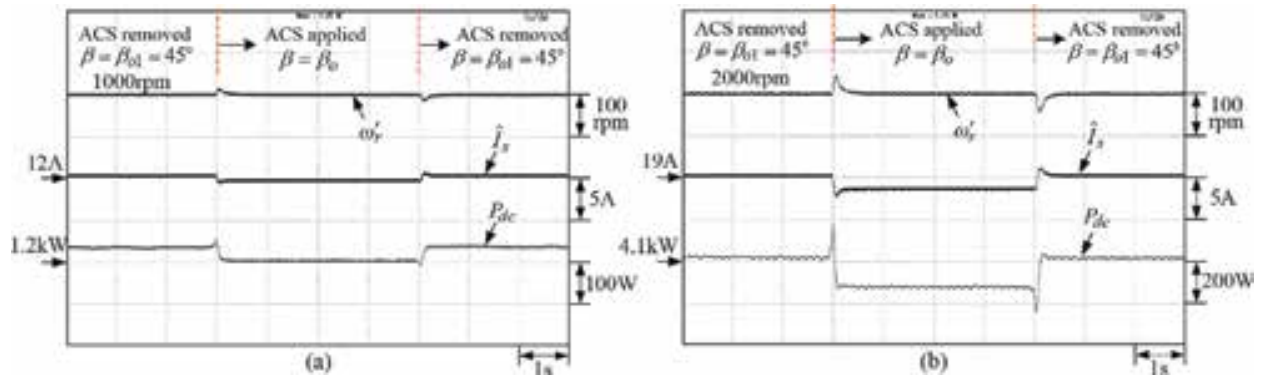

Figure 29.

Measured $\left(\omega_{r}^{\prime}, \hat{I}_{s}, P_{d c}\right)$ of the established SMR-fed SynRM drive with $\beta=\beta_{01}=45^{\circ}$ and $\beta=\beta_{o}$ by the proposed $A C S$ under $\left(V_{d c}=550 \mathrm{~V}, R_{L}=13.7 \Omega\right):(a) \omega_{r}^{\prime}=1000 \mathrm{rpm}$ and $(b) \omega_{r}^{\prime}=2000 \mathrm{rpm}$. 


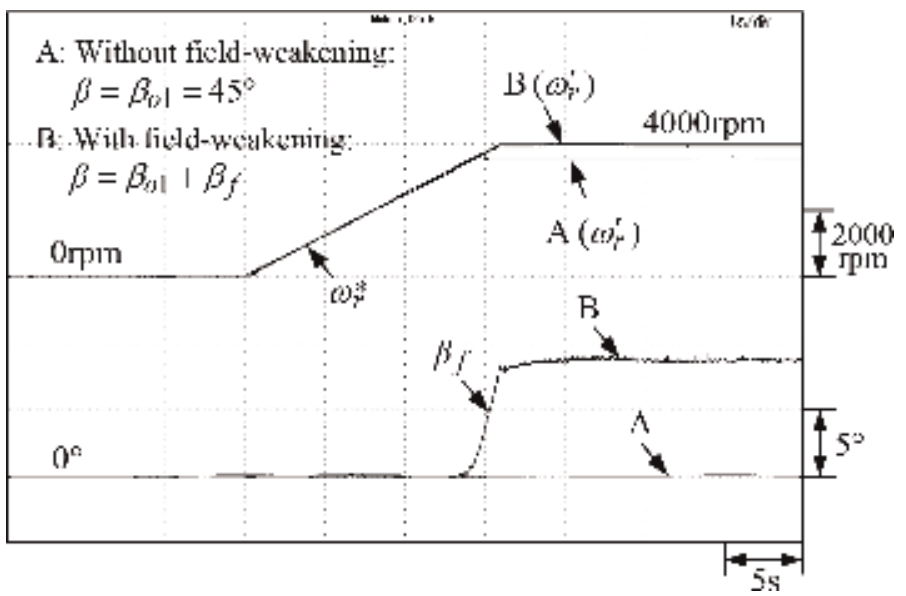

Figure 30.

Measured $\left(\omega_{r}^{*}, \omega_{r}^{\prime}\right)$ and the commutation angle $\beta_{f}$ yielded by the FWCS during starting process from o to $4000 \mathrm{rpm}$ at $\left(V_{d c}=550 \mathrm{~V}, R_{L}=303.9 \Omega\right)$.

speed command change $\omega_{r}^{*}=0 \rightarrow 2000 \mathrm{rpm} \rightarrow 0 \rightarrow-2000 \mathrm{rpm} \rightarrow 0$ with the rising and falling rates of $250 \mathrm{rpm} / \mathrm{s}$ being plotted in Figure 31. The results indicate the smooth speed tracking and reversible operation of the developed SynRM drive.

\subsubsection{Regenerative braking}

The SynRM drive is first stably operated at $\left(\omega_{r}^{\prime}=2000 \mathrm{rpm}, V_{d c}=550 \mathrm{~V}, R_{L}\right.$ $=\infty)$. Now the speed command is set from $\omega_{r}^{*}=2000 \mathrm{rpm}$ to $0 \mathrm{rpm}$ linearly in $1 \mathrm{~s}$. The measured DC-link voltage $v_{d c}\left(\omega_{r}^{*}, \omega_{r}^{\prime}\right), \hat{I}_{s}$ and phase-a winding current are of the established SMR-fed SynRM drive which are plotted in Figure 32a. One can be aware of the results that the current command $\hat{I}_{s}$ becomes negative under regenerative braking operation. Successful SynRG operation during regenerative braking is confirmed. And Figure $32 \mathrm{~b}$ shows the DC-link voltage $v_{d c}$, phase-A voltage $v_{A}$, and current $i_{A}$ of the mains. From Figure 32b, the regenerative braking of SynRM drive with energy recovered back to the mains can be observed.

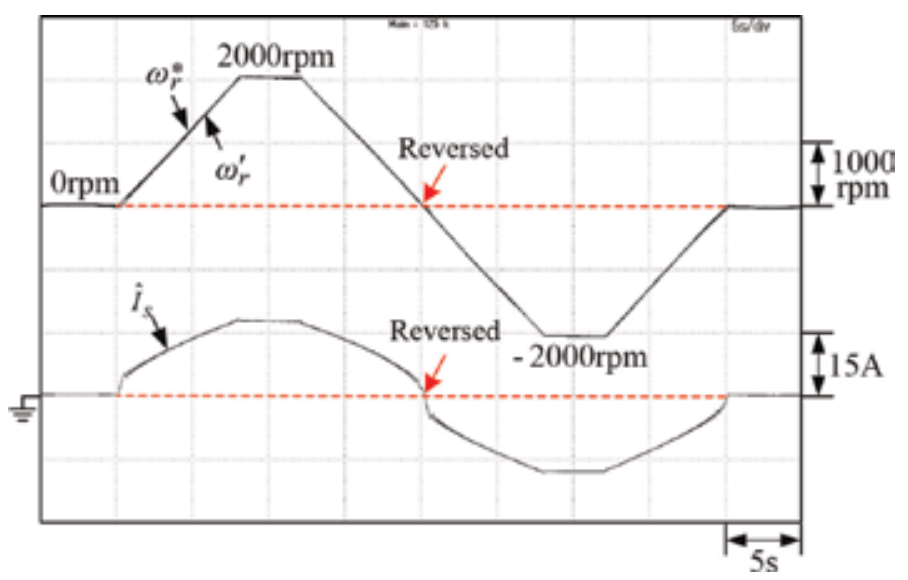

Figure 31.

Measured $\left(\omega_{r}^{*}, \omega_{r}^{\prime}\right)$ and $\hat{I}_{s}$ of the SynRM drive at $\left(V_{d c}=550 \mathrm{~V}, R_{L}=13.7 \Omega\right)$ with $\beta=\beta_{o}$ due to ramp speed command change $\omega_{r}^{*}=0 \rightarrow 2000 \mathrm{rpm} \rightarrow 0 \rightarrow-2000 \mathrm{rpm} \rightarrow 0$ with both the rising rate and falling rate being $250 \mathrm{rpm} / \mathrm{s}$. 

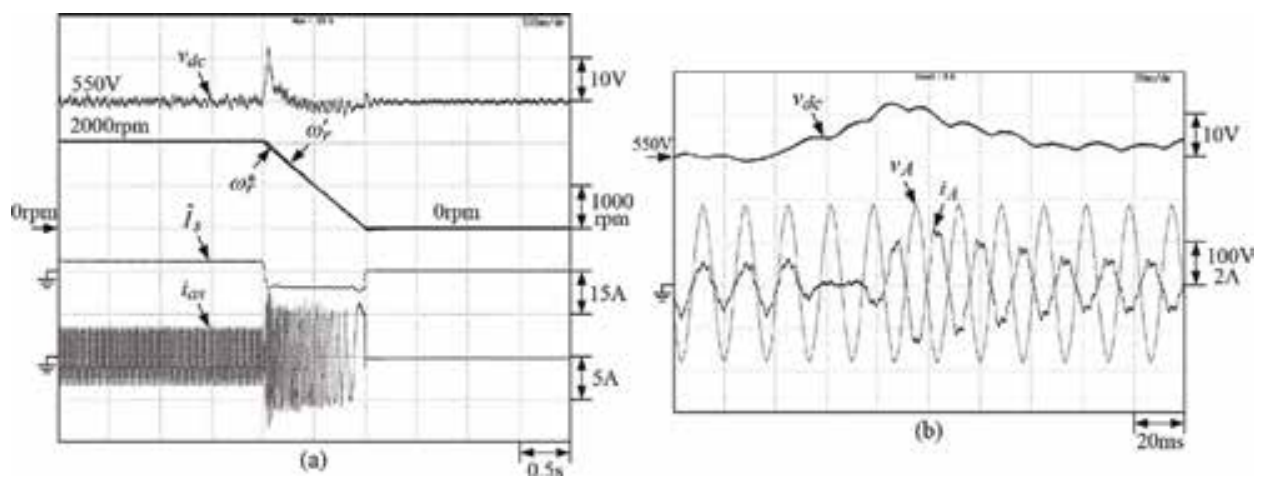

Figure 32.

Measured results of the established SMR-fed SynRM drive during braking by letting the speed command be changed from $\omega_{r}^{*}=2000 \mathrm{rpm}$ to o rpm linearly in $1 \mathrm{s:}$ (a) DC-link voltage $v_{d c}$, $\left(\omega_{r}^{*}, \omega_{r}^{\prime}\right)$, current command $\hat{I}_{s}$, and a-phase winding current, $i_{a s}$ and $(b)$ DC-link voltage $v_{d c}$, A-phase voltage, and current $\left(v_{A}, i_{A}\right)$ from the mains.

\subsubsection{Efficiency evaluation}

In order to verify the efficiency enhancement of the established SMR-fed SynRM drive using the proposed ACS, the measured steady-state characteristics at $\left(V_{d c}=550 \mathrm{~V}, R_{L}=13.7 \Omega, \omega_{r}^{\prime}=2000 \mathrm{rpm}\right)$ with the commutation angles $\beta=\beta_{o 1}=45^{\circ}$ and $\beta=\beta_{o}$ yielded by the proposed ACS are listed in Table 1 . The efficiencies are defined as:

$\eta_{S M R} \equiv P_{d c} / P_{a c}=$ SMR efficiency, $\eta_{m} \equiv$ SynRM efficiency, $\eta_{g} \equiv$ load PMSG efficiency, $\eta_{1} \equiv P_{L} / P_{d c}=\left(P_{m} / P_{d c}\right)\left(P_{g} / P_{m}\right)\left(P_{L} / P_{g}\right)$, and $\eta \equiv \eta_{S M R} \eta_{1}=P_{L} / P_{a c}=$ total efficiency.

The improvement in energy conversion efficiency via the proposed ACS is obvious from the results. The high efficiency possessed by the developed SynRM drive can also be aware.

\begin{tabular}{|c|c|c|c|c|c|c|}
\hline Speed & \multicolumn{3}{|c|}{$\beta=\beta_{o 1}=45^{\circ}$} & \multicolumn{3}{|c|}{$\beta=\beta_{o}$ by ACS } \\
\hline \multirow[t]{5}{*}{$2000 \mathrm{rpm}$} & \multicolumn{3}{|c|}{$P_{L}=3253.8 \mathrm{~W}$} & \multicolumn{3}{|c|}{$P_{L}=3251.7 \mathrm{~W}$} \\
\hline & \multicolumn{3}{|c|}{$P_{d c}=4064.3 \mathrm{~W}$} & \multicolumn{3}{|c|}{$P_{d c}=3980.1 \mathrm{~W}$} \\
\hline & \multicolumn{3}{|c|}{$P_{a c}=4380.0 \mathrm{~W}$} & \multicolumn{3}{|c|}{$P_{a c}=4285.0 \mathrm{~W}$} \\
\hline & \multirow{2}{*}{$\begin{array}{c}P F=0.998 \\
\eta_{S M R}=92.8 \%\end{array}$} & \multicolumn{2}{|c|}{$T H D_{i_{A}}=5.05 \%$} & $P F=0.997$ & \multicolumn{2}{|c|}{$T H D_{i_{A}}=4.98 \%$} \\
\hline & & $\eta_{1}=80.1 \%$ & $\eta=74.3 \%$ & $\eta_{S M R}=92.9 \%$ & $\eta_{1}=81.7 \%$ & $\eta=75.9 \%$ \\
\hline
\end{tabular}

Table 1.

Measured steady-state characteristics of the established SMR-fed SynRM drive at $\left(V_{d c}=550 \mathrm{~V}, R_{L}=13.7 \Omega, \omega_{r}^{\prime}=2000 \mathrm{rpm}\right)$.

\section{Conclusions}

As generally recognized, PMSM and SynRM are the two new motors having the potential to achieve the IE4-class and even the IE5-class efficiency. During the past years, the popularly used induction motors have been gradually replaced by these two motors in various applications, such as home appliances, EVs, wind generators, etc. For facilitating the development of high-performance PMSM and SynRM drives, this article has presented some basics and key affairs. Moreover, an example 
of EV PMSM drive and an example of SMR-fed SynRM drive have also been introduced for demonstration. Generally speaking, the most critical issues lie in (i) selecting suited type of motor for specific applications, (ii) proper commutation instant setting and shifting, (iii) applying DC-link voltage boosting approach to enhance high-speed driving characteristics, and (iv) properly conducting the regenerative braking operation control.

\section{Author details}

Chang-Ming Liaw*, Jia-Hsiang Zhuang, Shih-Wei Su, Kai-Wei Hu and G. Vijay Kumar

Department of Electrical Engineering, National Tsing Hua University, Hsinchu, Taiwan, ROC

*Address all correspondence to: cmliaw@ee.nthu.edu.tw

\section{IntechOpen}

(C) 2019 The Author(s). Licensee IntechOpen. This chapter is distributed under the terms of the Creative Commons Attribution License (http://creativecommons.org/licenses/ by/3.0), which permits unrestricted use, distribution, and reproduction in any medium, provided the original work is properly cited. (c) BY 


\section{References}

[1] Krause PC, Wasynczuk O, Sudhoff SD. Analysis of Electric Machinery and Drive System. 2nd ed. New York: WileyIEEE; 2002

[2] Murakami H, Honda Y, Kiriyama H, Morimoto S, Takeda Y. The performance comparison of SPMSM, IPMSM and SynRM in use as airconditioning compressor. In: Proceedings of IEEE IAS; 1999. pp. 840-845

[3] Murakami H, Honda Y, Morimoto S, Takeda Y. Permanent magnet assisted synchronous reluctance motor. IEEJ Transactions on Industry Applications. 2002;122(3):266-272

[4] Zeraoulia M, Benbouzid MEH, Diallo D. Electric motor drive selection issues for HEV propulsion systems: A comparative study. IEEE Transactions on Vehicular Technology. 2006;55(6): 1756-1764

[5] Morimoto S. Trend of permanent magnet synchronous machines. IEEJ Transactions on Electrical and Electronic Engineering. 2007;2(2): 101-108

[6] Pellegrino G, Vagati A, Boazzo B, Guglielmi P. Comparison of induction and PM synchronous motor drives for EV application including design examples. IEEE Transactions on Industry Applications. 2012;48(6): 2322-2332

[7] Bostanci E, Moallem M, Parsapour A, Fahimi B. Opportunities and challenges of switched reluctance motor drives for electric propulsion: A comparative study. IEEE Transactions on Transportation Electrification. 2017; 3(1):58-75

[8] Weisgerber S, Proca A Keyhani A. Estimation of permanent magnet motor parameters. Proceedings of IEEE IAS. 1997;1(1):29-34
[9] Kondo M. Parameter measurements for permanent magnet synchronous machines. IEEJ Transactions on Electrical and Electronic Engineering. 2007;2(2):109-117

[10] Kazmierkowski MP, Malesani L. Current control techniques for three phase voltage-source PWM converters: A survey. IEEE Transactions on Industrial Electronics. 1998;45(5): 691-703

[11] Chou MC, Liaw CM, Chien SB, Shien FH, Tsai JR, Chang HC. Robust current and torque controls for PMSM driven satellite reaction wheel. IEEE Transactions on Aerospace and Electronic Systems. 2011;47(1):58-74

[12] Siami M, Khaburi DA, Abbaszadeh A, Rodriguez J. Robustness improvement of predictive current control using prediction error correction for permanent-magnet synchronous machines. IEEE Transactions on Industrial Electronics. 2016;63(6): 3458-3466

[13] Sawma J, Khatounian F, Monmasson E, Idkhajine L, Ghosn R. Analysis of the impact of online identification on model predictive current control applied to permanent magnet synchronous motors. IET Electric Power Applications. 2017; 11(5):864-873

[14] Preindl M, Bolognani S. Model predictive direct speed control with finite control set of PMSM drive systems. IEEE Transactions on Power Electronics. 2013;28(2):1007-1015

[15] Jung JW, Leu VQ, Do TD, Kim EK, Choi HH. Adaptive PID speed controller design for permanent magnet synchronous motor drives. IEEE Transactions on Power Electronics. 2015;30(2):900-908

[16] Sant AV, Rajagopal KR. PM synchronous motor speed control using 
hybrid fuzzy-PI with novel switching functions. IEEE Transactions on Magnetics. 2009;45(10):4672-4675

[17] Lukichev DV, Demidova GL. Speed control in PMSM drive with non-stiff load and unknown parameters using PI -And fuzzy adaptive PID controllers. In: 2017 International Conference on Industrial Engineering, Applications and Manufacturing (ICIEAM); 2017. pp. 1-5

[18] Justo JJ, Mwasilu F, Kim EK, Kim J, Choi HH, Jung JW. Fuzzy model predictive direct torque control of IPMSMs for electric vehicle applications. IEEE/ASME Transactions on Mechatronics. 2017;2(4):1542-1553

[19] Mohamed YA-RI, Lee TK. Adaptive self-tuning MTPA vector controller for IPMSM drive system. IEEE Transactions on Energy Conversion. 2006;21(3): 636-644

[20] Niazi P, Toliyat HA, Goodarzi A. Robust maximum torque per ampere (MTPA) control of PM-assisted SynRM for traction applications. IEEE Transactions on Vehicular Technology. 2007;56(4):1538-1545

[21] Chen HC Liaw CM. Sensorless control via intelligent commutation tuning for brushless DC motor.

Proceedings of IEE Electric Power Applications. 1999;146(6):678-684

[22] Chen HC, Liaw CM. Current-mode control for sensorless BDCM drive with intelligent commutation tuning. IEEE Transactions on Power Electronics. 2002;17(5):747-756

[23] Liaw CC, Liaw CM, Chang HC, Huang MS. Robust current control and commutation tuning for an IPMSM drive. Proceedings of IEEE APEC. 2003; 2(2):1045-1051

[24] Sato E. Permanent magnet synchronous motor drives for hybrid electric vehicles. Transactions on Electrical and Electronic Engineering. 2007;2(2):162-168

[25] Bolognani S, Calligaro S, Petrella R. Adaptive flux-weakening controller for interior permanent magnet synchronous motor drives. IEEE Journal of Emerging and Selected Topics in Power Electronics. 2014;2(2):236-248

[26] Burress T. Benchmarking EV and HEV technologies. Technical Report ORNL; 2015

[27] Chou MC, Liaw CM. PMSM-driven satellite reaction wheel system with adjustable DC-link voltage. IEEE Transactions on Aerospace and Electronic Systems. 2014;50(2): 1359-1373

[28] Liaw CM, Hu KW, Lin YS, Yeh TH. An electric vehicle IPMSM drive with interleaved front-end DC/DC converter. IEEE Transactions on Vehicular Technology. 2016;65(6):4493-4504

[29] Garcia O, Cobos JA, Prieto R, Alou P, Uceda J. Single phase power factor correction: A survey. IEEE Transactions on Power Electronics. 2003;18(3):

749-755

[30] Singh B, Singh NB, Chandra A, Haddad KA, Pandey A, Kothari PD. A review of three-phase improved power quality AC/DC converters. IEEE Transactions on Industrial Electronics. 2004;51(3):641-660

[31] Friedli T, Kolar JW. The essence of three-phase PFC rectifier systems part I. IEEE Transactions on Power Electronics. 2013;28(1):176-198

[32] Yilmaz M, Krein PT. Review of battery charger topologies, charging power levels, and infrastructure for plug-in electric and hybrid vehicles. IEEE Transactions on Power Electronics. 2013;28(5):2151-2169 
[33] Matsui N. Sensorless PM brushless DC motor drives. IEEE Transactions on Industrial Electronics. 1996;43(2): 300-308

[34] Johnson JP, Ehsani M, Guzelgunler Y. Review of sensorless methods for brushless DC. Proceedings of IEEE IAS. 1999;1:143-150

[35] Sul SK, Kwon YC, Lee Y. Sensorless control of IPMSM for last 10 years and next 5 years. CES Transactions on Electrical Machines and Systems. 2017; 1(2):91-99

[36] Hamida MA, Leon JD, Glumineau A, Boisliveau R. An adaptive interconnected observer for sensorless control of PM synchronous motors with online parameter identification. IEEE Transactions on Industrial Electronics. 2013;60(2):739-748

[37] Rafaq MS, Mwasilu F, Kim J, Choi $\mathrm{HH}$, Jung JW. Online parameter identification for model-based sensorless control of interior permanent magnet synchronous machine. IEEE Transactions on Power Electronics. 2017;32(6):4631-4643

[38] Jang JH, Ha JI, Ohto M, Ide K, Sul SK. Analysis of permanent-magnet machine for sensorless control based on high-frequency signal injection. IEEE Transactions on Industry Applications. 2004;40(6):1595-1604

[39] Guerrero JM, Leetmaa M, Briz F, Zamarron A, Lorenz RD. Inverter nonlinearity effects in high-frequency signal-injection-based sensorless control methods. IEEE Transactions on Industry Applications. 2005;41(2):618-626

[40] Raca D, Garcia P, Reigosa DD, Briz F, Lorenz RD. Carrier-signal selection for sensorless control of PM synchronous machines at zero and very low speeds. IEEE Transactions on Industry Applications. 2010;46(1): 167-178
[41] Andreescu GD Schlezinger C.

Enhancement sensorless control system for PMSM drives using square-wave signal injection. In: Proceedings of IEEE SPEEDAM. 2010. pp. 1508-1511

[42] Kim D, Kwon YC, Sul SK, Kim JH, $\mathrm{Yu}$ RS. Suppression of injection voltage disturbance for high-frequency squarewave injection sensorless drive with regulation of induced high-frequency current ripple. IEEE Transactions on Industry Applications. 2016;53(1): 302-312

[43] Po-ngam S, Sangwongwanich S. Stability and dynamic performance improvement of adaptive full-order observers for sensorless PMSM drive. IEEE Transactions on Power Electronics. 2012;27(2):588-600

[44] Park Y, Sul SK. Sensorless control method for PMSM based on frequencyadaptive disturbance observer. IEEE Journal of Emerging and Selected Topics in Power Electronics. 2014;2(2):143-151

[45] Hejny RW, Lorenz RD. Evaluating the practical low-speed limits for backEMF tracking-based sensorless speed control using drive stiffness as a key metric. IEEE Transactions on Industry Applications. 2011;47(3):1337-1343

[46] Sarikhani A, Mohammed OA. Sensorless control of PM synchronous machines by physics-based EMF observer. IEEE Transactions on Energy Conversion. 2012;27(4):1009-1017

[47] Antonello R, Ortombina L, Tinazzi F, Zigliotto M. Enhanced low-speed operations for sensorless anisotropic PM synchronous motor drives by a modified back-EMF observer. IEEE Transactions on Industrial Electronics. 2018;65(4): 3069-3076

[48] Hideaki I, Masanobu I, Takeshi K Kozo I. Hybrid sensorless control of IPMSM for direct drive applications. In: 
Proceedings of IEEE IPEC; 2010. pp. 2761-2767

[49] Lara J, Chandra A, Xu J. Integration of HFSI and extended-EMF based techniques for PMSM sensorless control in HEV/EV applications. In: 2012 Annual Conference on IEEE Industrial Electronics Society (IECON); 2012. pp. $3688-3693$

[50] Lipo TA. Synchronous reluctance machine-A viable alternative for AC drive. Electric Machine \& Power System. 1991;19(6):659-671

[51] Matsuo T, Lipo TA. Rotor design optimization of synchronous reluctance machine. IEEE Transactions on Energy Conversion. 1994;9(2):359-365

[52] Bianchi N, Bolognani S, Carraro E, Castiello M, Fornasiero E. Electric vehicle traction based on synchronous reluctance motors. IEEE Transactions on Industry Applications. 2016;52(6): 4762-4769

[53] Qu Z, Hinkkanen M. Lossminimizing control of synchronous reluctance motors-A review. Proceedings of IEEE ICIT; 2013. pp. 350-355

[54] Lubin T, Razik H, Rezzoug A. Magnetic saturation effects on the control of a synchronous reluctance machine. IEEE Transactions on Energy Conversion. 2002;17(3):356-362

[55] Hofmann HF, Sanders SR, ELAntably A. Stator-flux-oriented vector control of synchronous reluctance machines with maximized efficiency. IEEE Transactions on Industrial Electronics. 2004;51(5):1066-1072

[56] Zarchi HA, Soltani J, Markadeh GRA, Fazeli M, Sichani AK. Variable structure direct torque control of encoderless synchronous reluctance motor drives with maximized efficiency. In: Proceedings of IEEE ISIE; 2010. pp. 1529-1535
[57] Kim S, Sul SK, Ide K, Morimoto S. Maximum efficiency operation of synchronous reluctance machine using signal injection. In: Proceedings of IEEE ECCE; 2010. pp. 2000-2004

[58] Inoue Y, Morimoto S, Sanada M. A novel control scheme for maximum power operation of synchronous reluctance motors including maximum torque per flux control. IEEE

Transactions on Industry Applications. 2011;47(1):115-121

[59] Yamamoto S, Hirahara H, Adawey JB, Ara T, Matsuse K. Maximum efficiency drives of synchronous reluctance motors by a novel loss minimization controller with inductance estimator. IEEE Transactions on Industry Applications. 2013;49(6):2543-2551

[60] Varatharajan A, Cruz S, Hadla H, Briz F. Predictive torque control of SynRM drives with online MTPA trajectory tracking and inductances estimation. In: Proceedings of IEEE IEMDC; 2017. pp. 1-7

[61] Bedetti N, Calligaro S, Petrella R. Self-adaptation of MTPA tracking controller for IPMSM and SynRM drives based on on-line estimation of loop gain. In: Proceedings of IEEE ECCE; 2017. pp. 1917-1924

[62] Mahmoud H, Bianchi N. Nonlinear analytical model of eccentric synchronous reluctance machines considering the iron saturation and slotting effect. IEEE Transactions on Industry Applications. 2017;53(3):2007-2015

[63] Wang KC. A battery/supercapacitor powered EV PMSM drive with grid connected and energy harvesting functions [thesis]. Hsinchu, ROC: Department of Electrical Engineering, National Tsing Hua University; 2017

[64] Su SW. Development of a synchronous reluctance motor drive and 
its position sensorless control study [thesis]. Hsinchu, ROC: Department of Electrical Engineering, National Tsing Hua University; 2017

[65] Zhuang JX, Development of position sensorless synchronousreluctance generator system and its performance enhancement controls [thesis]. Hsinchu, ROC: Department of Electrical Engineering, National Tsing Hua University; 2018 


\title{
Mechanical and Electrical Design Calculations of Hybrid Vehicles
}

\author{
Khalid G. Mohammed
}

\begin{abstract}
Electric power is widely used in electric traction for many reasons: it is easy to control the speed of an electric motor, the absence of exhaust gases, free of noise, it has high starting torque, and it needs less maintenance than its mechanical counterpart. In the current research, a modern hybrid car is designed and manufactured in three ways. The first method is using a 220 volt AC electric power to charge five series batteries; each battery has 12 VDC ( $35 \mathrm{~A} / \mathrm{h}$ ) to supply totally $60 \mathrm{VDC}$ input voltage for the electronic inverter which converts 60 VDC to 60 VAC (three-phase voltage) as a controllable voltage source to three-phase synchronous motor (SM) type (BLDC-YG1-ZZ-1200 W). The second method is to take advantage of the solar energy which is almost available in Iraq environment throughout the year to be stored in the batteries, especially during the shutdown of the machine and when it stops it under the sun. The solar panel is fixed on the vehicle's roof; it has a power of 100 watts. The third method is the mechanical energy by using the bicycle pedal to move the wheels of the car; it is useful in the event of a sudden interruption of electrical power or a technical failure in the vehicle. In addition, three kinds of electronic devices are used for control. The first control is electric battery charger. The second control is to convert solar radiation into electrical energy to be stored in the batteries. The third control is to regulate the accessories of another electric vehicle. The vehicle was tested in the province of Diyala, Baquba, Iraq, on a flat and tilted land (Al Mafraq Bridge, Baquba city). The steady-state speed reached more than $40 \mathrm{~km} / \mathrm{h}$ with a total load of more than $125 \mathrm{~kg}$. The design is subjected to real electrical and mechanical engineering tests alongside using decades of applied equations on locomotives and electric vehicles to validate the experimental tests.
\end{abstract}

Keywords: hybrid vehicle, electrical, solar and mechanical energies

\section{Introduction}

Electric cars as many people have seen have acquired a great significance because it is an alternative future way for transmission due their features. In spite of that, there are three difficulties that face the evaluation of the electric cars: long time of charging, cost of batteries, and limited driving ranges [1-3]. The great developments in power electronic inverters which are used as a main controller inside the electric cars have given a step frontward [4, 5]. In addition to that, the electric machine motors such as DC motors, induction motors, and three-phase synchronous motors have witnessed effective process in material and electromagnets aided to increase their efficiency [6-8]. 
The traction motors need strongly two important parameters to recognize their performance: high starting torque and acceleration. Furthermore, the traction motor should have the initial force to overcome the resistance force induced at the starting movement $[9,10]$.

The electric car was first invented in the USA and Scotland in 1834. After that, slow progress began to prove its importance until three eras of development to this day. These three boom eras were dominated by factors such as the presence of oil, prices, and crises, and the second factor is the pollution of the environment and the increase in temperature of the earth.

The first boom era began in 1850 and continued to 1900 in the USA where the DC motors were the basic component of the drag with the presence of batteries. The second boom era started from 1950 to 2000, where the 1973 oil crisis, pollution of the environment, and global warming contributed to this development. The third boom started since the beginning of 2000 to the present day, where the Japanese companies, Toyota and Nissan, and the German company, Mercedes or BMW, and Chinese companies as well as US companies entered in a fierce competition to control the global markets $[11,12]$. Table 1 shows the history of the electric car since 1834 until these days.

\begin{tabular}{|c|c|c|c|}
\hline Name & $\begin{array}{l}\text { Year and } \\
\text { country }\end{array}$ & Type of traction & Notes \\
\hline Thomas Davenport & 1834, USA & DC motor & \\
\hline Robert Anderson & $\begin{array}{l}\text { 1832-1839, } \\
\text { Scotland }\end{array}$ & $\begin{array}{l}\text { First prototype electric-powered } \\
\text { carriage }\end{array}$ & \\
\hline Andreas Flocken & $\begin{array}{c}\text { 1888, } \\
\text { Germany }\end{array}$ & The first four-wheeled electric car & \\
\hline $\begin{array}{l}\text { Pope Manufacturing } \\
\text { Co. }\end{array}$ & 1897, USA & The first commercial electric vehicle & \\
\hline \multirow[t]{2}{*}{ La Jamais Contente } & 1899, France & $\begin{array}{l}\text { The first electric vehicle to travel over } \\
\qquad 100 \mathrm{~km} \text { per hour }\end{array}$ & \\
\hline & 1900, USA & Electricity-powered cars & $\begin{array}{l}\text { Capturing } 28 \% \text { of } \\
\text { the market }\end{array}$ \\
\hline $\begin{array}{l}\text { The petrol-powered } \\
\text { Ford Model }\end{array}$ & 1908, USA & Electric car Model T & \\
\hline A Baker Electric & 1909, USA & Automobile & \\
\hline Charles Kettering & 1912, USA & $\begin{array}{l}\text { The electric starter was invented } \\
\text { instead of hand-crank }\end{array}$ & \\
\hline Global EV stock & 1912, USA & & Market, 30,000 \\
\hline Carmaker Tama & 1947, Japan & $\begin{array}{l}\text { Electric car with a } 40 \mathrm{~V} \text { lead } \\
\text { acid battery }\end{array}$ & \\
\hline General Motors & 1996, USA & EV1 electric car & \\
\hline Toyota & 1997, Japan & $\begin{array}{l}\text { The Prius, the world's first commercial } \\
\text { hybrid car }\end{array}$ & \\
\hline BEV Nissan & 2010, Japan & $\begin{array}{c}\text { The world's largest electric car sharing } \\
\text { service }\end{array}$ & \\
\hline Nissan LEAF & 2011, Japan & New car models & \\
\hline PHEV Chevrolet Volt & 2012, USA & New car models & \\
\hline
\end{tabular}

Table 1.

Electric car history [12]. 


\subsection{Electric traction motors}

\subsubsection{Traction motor types}

There are three types of electric motors which are nominated to use: DC motor, AC single- or three-phase induction motor (IM), and three-phase synchronous motor (SM). These motor features are chosen depending on the nature of voltage-supplied source, performance, and method of construction.

\subsubsection{DC motors}

DC motors are characterized as follows: exacted spin speeds, the same wide range of changing speed with the possibility of easy control, the possibility of reversing the rotational movement, and the possibility to start an appropriate torque.

\subsubsection{Induction motors}

There are two types of induction motors: the first type has a rotor shape-like squirrel cage arrangement. It is characterized by simple installation, low cost, and hardness. This type is suitable for applications requiring constant rotation speed. The second type is called the slip-ring motor which has a wound rotor with a threephase winding. The first three terminals of the windings are shorted, while the second three ending terminals of the windings are connected to the three slip rings. Slip-ring induction motor is suitable for applications that need great starting torque for a few seconds with a decrease in the value of the starting current. This type is characterized by the first type with its great ability to control the speed and torque and the starting current by using additional variable three-phase resistance connected through the three slip rings of the motor.

\subsubsection{Synchronous motors}

This type of motor is suitable for applications that need a constant rotation speed value and great power in addition to the possibility designed to operate at slow speeds by a high power factor and high operating efficiency.

\subsection{General characteristics of traction motors}

The general electrical mechanical properties required for the traction motors are:

1. A large starting torque and acceleration which are needed by electric vehicle to overcome the power large traction required at starting the movement. In addition to the traction force needed to overcome the resistance to train traffic, also, the movement toward the highest inclined land means the need to extra drag to overcome the effect of gravity.

2. Series motor properties: It is necessary for the motor used in the vehicle to have series motor properties between motor speed and torque, for the following reasons:

- Operating more than one motor per electric vehicle: Electric vehicle usually contains several similar traction motors which are installed with movement gears and wheels. As long as the wheels of movement have the 
same diameter, the motor speeds are equal, and the entire load is equally distributed between the motors. But because of unequal corrosion that happens, there is a dissimilarity in the wheels' diameters. This may cause an unequal distribution of the load between the motors.

- Self-protection feature: Because of the characteristics between the speed and torque, the speed of the motor decreases as the torque increases. This relationship is correct when neglecting magnetic saturation.

- Lower power consumption during load increase: During load increase, the required traction increases, as is the case with a sloping level, when the required power by the series motor is much lower than the power calculated by the parallel motor; the reasons are mentioned above.

\subsection{Advantages and disadvantages of electric traction}

The most important advantages of using the electric motors in traction are anticontamination that accompanies with the use of electric motors. The electric motor provides a great starting torque, which allows high acceleration value at the start of the vehicle. So, they allow carrying twice as many people on the same way because of the high flow of vehicle speed. The electric motor provides a soft change in the ultrafast speed.

The electric motor provides the possibility of using the electric brake, which enables the return power to the electric grid when using the brake regeneration while walking down the slopes. The use of electric brakes leads to savings in the use of the mechanical brakes which prolongs life and reduces corrosion in the roller wheels and iron bars. The time required for the maintenance and repair of an electric vehicle is lesser than the need by others. The maintenance and repair of the electric vehicle cost about half of those cost in other vehicles. They did not need electric vehicles to the time to become operational. Finally, getting rid of the exhaust fumes, which may contain toxic elements, is considered one of the most important advantages of electric traction, especially in hypocrisy and roads under the ground. The disadvantages of electric traction are as follows: The cost of construction is high, any malfunction in the electrical grid even for a brief period will lead to total paralysis in traffic and might extend long hours, and also an overlap occurs between the electric traction network and communication signals.

\subsection{Feeding electric traction system networks}

There are three different types of feed electric traction systems:

a. DC voltage system (DC power system)

b. Single-phase AC voltage system

c. Three-phase AC voltage system

(a) DC power system: DC voltage of 600 to $700 \mathrm{~V}$ is used globally for trams in the cities, while the ongoing effort of the $1500-3000 \mathrm{~V}$ is used outside the cities. The iron bars represent the neutral line. The specification states that the voltage drop should not be more than 7 volts between any two points on the neutral line (iron bars). 
As for the electric bus (trolleybus), the two electric lines should be two overhanged, the feeding line and neutral line. The voltage must not exceed $10 \%$ plus or minus. The DC network is fed into electric power stations 3-5 kilometers away from each other inside the cities and 40-50 kilometers outside the cities. Electric power stations are also supplied with power from the $\mathrm{AC}$ voltage networks of $110-122 \mathrm{kV}$, where it is converted into a DC voltage required.

(b) Single-phase AC system: This system uses a voltage of $11-15 \mathrm{kV}(2 / 316)$ or $25 \mathrm{~Hz}$. If power plants are used to feed traction stations, there will be no difficulty in generating the required voltage and frequency. In the case of high-voltage networks at 50 or $60 \mathrm{~Hz}$ frequency, the frequency limit should be reduced to the desired limit (a three-phase synchronous motor is used to operate a single-phase generator to generate the required voltage and frequency).

In this case, the traction network consists of a single overhanged line, and the rails represent the neutral. The traction line carries the transformer to reduce the voltage to 300 or 400 volts to feed the general series motor, and the motor speed can be controlled by changing the voltage of the transformer.

Low frequency (16.667) or $25 \mathrm{~Hz}$ is used to improve the efficiency and power factor of the motor. It also helps to reduce the electrical spark between the brushes and the commutator, in addition to reducing the induction impedance of the traction network transmission lines and thus reducing the lost voltage. This increases the distance between feeding stations to 50-80 kilometers. Low-frequency use also helps reduce interference with telephone and communication networks.

(c) Three-phase AC system: Three-phase induction motors are used as drive motors in this case with a voltage of $3.3 \mathrm{kV}$ and a frequency of $16.667 \mathrm{~Hz}$. The power stations receive power from high-voltage networks, and the voltage and frequency are reduced to the required limit. The traction network consists of two transmission lines; the line bar is the third line. The advantages mentioned, due to the use of low frequency, can also be mentioned in this system.

\subsection{A factor that must be considered when choosing an electric motor}

Choosing an electric motor depends on the circumstances that will work underneath and the type of load. There are several factors to consider when choosing an electric motor to suit industrial applications, and one of the most important are as follows:

1. Electrical properties:

a. The properties of the start of the movement, in terms of the value of each of the torque and the drawn current

b. The properties of the motor during operation, the relationship between the torque and speed, and the relationship between speed and power, current, losses, and efficiency

c. The extent of control the speed of rotation during operation

d. How to turn off the motor and break it

2. Mechanical considerations:

a. The type of outside cover of the motor, the type of cooling of the motor.

b. The type of rotary bearings used. 
c. What is the transmission way between the motor and the load.

d. The noise level, which is produced by the motor.

3. The motor size and the design power:

a. Loading requirements in terms of continuous or short term or intermittent

b. Ability of the motor to deal with excessive loads

4. The cost of the motor:

a. In terms of the primary and operating cost

In addition to the previous factors, it should be kept in mind the current user type, in terms of being a DC constant, AC single-phase, or three-phase current. From the above it is clear that there are many factors must be considered when choosing a motor to drive a given load, and despite the fact that the cost of the machine came in the last previous mechanical considerations, but the final decision in the selection of the machine, depending upon significantly. The desired and selected machine must meet all the technical requirements of the load and at the same time should not be so high till it succeeds economically. In fact, the choice of the motor requires careful study and analysis of its characteristics and load together.

In addition to the full knowledge of the entire system of stirring and control devices, it is required to have switching devices and change the frequency.

The latest factors and their impact on different types of motors will be discussed in detail separately in the next section, to illustrate the impact of each of them:

1. Electrical properties:

The properties of the different motors in terms of operating properties and the properties of the start of the movement and speed control have been studied in the decisions of the DC machines and AC small power motors.

2. Mechanical considerations:

A. Cover user type: The main objective of the outer frame of the machine is not only to provide protection for the people and the workers but also to provide protection for the machine itself, against moisture, dirt, dust, and unwanted objects and what might leak out of the motor fumes and flammable materials. There are different types of the motors according to their protection ways:

i. Open type

ii. Mesh cover-protected type

iii. Type covered against scattering of liquids and dust

iv. Water- and rain-protected type

v. Self-air cooling 
vi. Separated cooling

vii. Tubed cooling

B. Bearings used type:

There are two types of bearings used in electric motors, namely, ball bearings and roller bearings.

i. Ball bearings: used in motors up to 100 horsepower. They are preferred by other types due to its many benefits. The most important of these are little friction losses, less maintenance, and longer durability than the other wheels. Between stator and rotor. But its main disadvantages are its high cost and noise, especially at high speeds.

ii. Roller bearings: used in motors that work in quiet places such as hospitals, offices, and classrooms.

C. Types of transmission: Transfer the generated mechanical power to the machine to drive the mechanical load axis; there are many ways to lead the load, and the most important are:

i. Direct transmission: The load and the motor are directly connected by a mechanical coupling which is solid or flexible. Coupling is used when the load speed is equal to the speed of the motor.

ii. Driving by timing belts to transfer power of up to two hundred and fifty kilowatts, preferably when using this method to be less distance between the axis of rollers equal to four to five times the diameter of the largest pulley, and so that the maximum ratio between the diagonal rollers are 1: 6 , as there is in this case sliding between the borders of three to four percent. The disadvantage of this method is that it requires a large space, so the belts make tension sideways on the bearings, causing increased friction missing out and fatigue.

iii. Driving by using V-type belts: This type of V-type conveyor is used between two rollers with the same shape. This method is used to transport large valves that exceed the capacity of the belt, and it operates with a small slip that can be neglected.

iv. By driving chains: This method is more efficient and is used at high speeds, but the cost is the biggest. But they need less space than the previous two, where the required distance between the axis of rollers is of one and half to twice the diameter of a larger pulley. It is used in wet and dust places so that the chains should be protected by its own cover, as it should have perfectly parallel axes, to avoid lateral tensile axis rollers.

v. By steering with gearbox: This method is used when the motor used has a high speed to drive a load when its speed is slow; the 
motor is built and merged with gearbox by the required speed ratio.

D. The noise produced by the motor: noise produced inside the motor for the following reasons:

i. Alternating magnetic field inside the motor caused by vibrations in the iron body segments and the motor

ii. The movement of air within the instrument

iii. Friction in the bearings

Noise level should be reduced to the lowest level possible, especially in motors that are used in hospitals, offices, theaters, and classrooms. To reduce the transitional noise from the motor to the other places, it must be installed with rubber or helical springs to absorb vibrations.

3. Size of the motor and its power:

The factors that control the size and capacity of the motor is the maximum temperature reached by the motor during the service run under load conditions, in that it continuously or intermittently, or short-term and the maximum. It has been found that the motor that achieves the first condition of temperature also achieves the second condition of the torque required. It is worth mentioning here that the maximum temperature of the motor is designed on the basis of the type of the insulating material used. Motor insulations are classified to types depending on the maximum temperature allowed.

Loading requirements in terms of that continuous or short term or intermittent: usually electric motors are designed based on the amount of time in which they operate the machine required load as well as the amount of time in which the machine is stationary for work or runs at no load. On this basis, the rules that follow to choose electric motors indicate the possibility of rating motor in terms of time plan. Here are some operating types of motor:

A. Continuous operation: the machine working needs to do its job in this case to start the motor full rating and on continuous so as to reach the temperature in all parts of the motor to the maximum amount at which the motor has been designed on the basis of not beyond it with the operating continuation of any period after that.

B. Short-term operating: The machine working in this type of operation runs sporadic periods, each of which extends over a specific period of time, the measured temperature so as not to exceed in all parts of the motor the maximum temperature.

\section{Methodology: design of vehicle}

The required torque for moving vehicle gear box assembly is subjected to many rules. These rules are interrelated with tires, friction, wind resistance, weight, and tilt level [4]. 


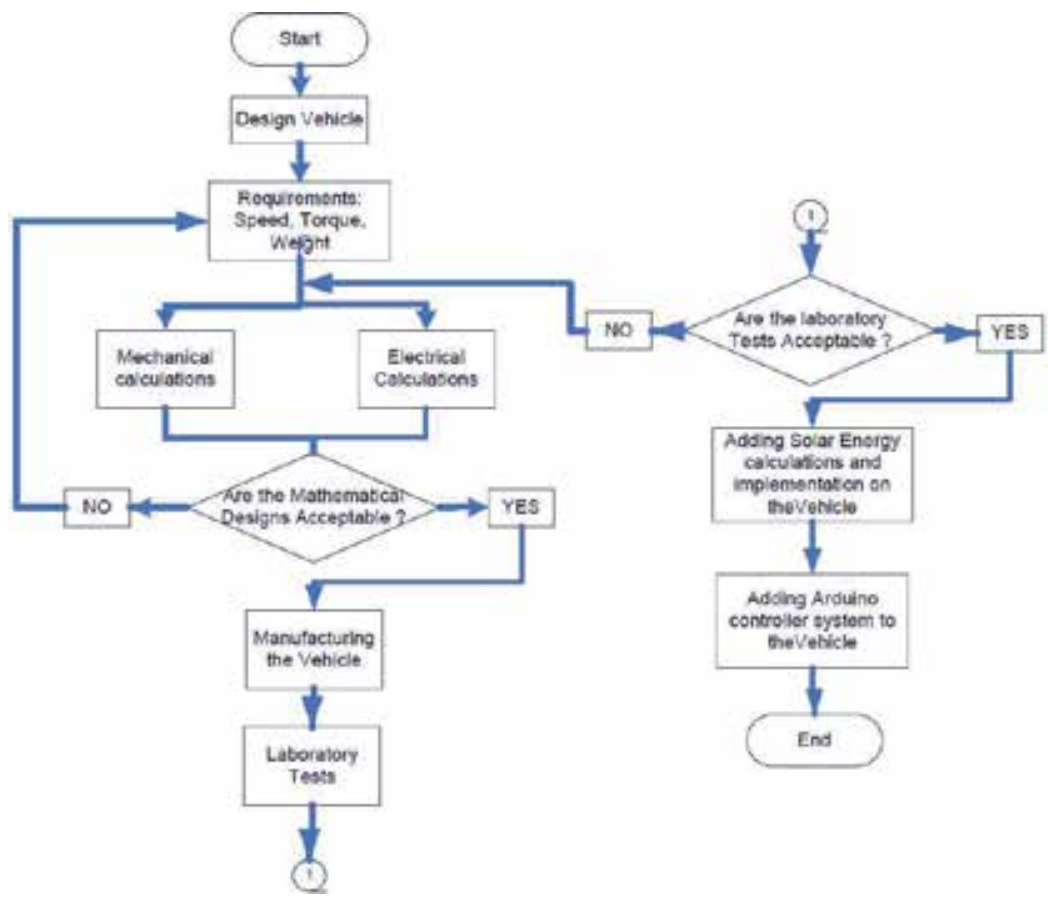

Figure 1.

Flowchart of the vehicle design.

A tire subjected to a load will be deformed and flattened until the contact area with the ground will get a force equal to the load on the same wheel. When the tire rolls, the resulting force at contact is not centered but placed at a distance: this is the lever arm required to calculate the required torque on the tire to overcome the resistance by rolling (it is not the tire radius). The friction coefficient between the tire rubber and ground is only required to find out the maximum torque before sliding. This may occur in case of a very high starting torque when the moment of vehicle inertia has an essential role. Tire deformation demands energy, and tires have an internal friction in which it is clear that after driving, tires heat up. The motor must supply the torque and energy to overcome all losses. If, for instance, two wheels are active, the rolling resistance and other losses are present on all four wheels even if the torque is applied only on two. Since rolling resistances are not linear, their sum should consider load distribution on the different axes and wheels. All before is valid for a flat horizontal ground. When the road is inclined with an angle, then the $\mathrm{e}=$ weight force can be analyzed to components. The downward component is $\mathrm{F}=\mathrm{G} \times$ sin (angle). The friction coefficient also adds an opposite torque to the tire torque. Figure 1 explains the design flowchart of the vehicle.

\subsection{Mechanical model}

The required traction force on the tire of the vehicle is $F_{t}$. The force $F_{t}$ induced on the edge of the vehicle tires is to move it itself. The force traction required for the vehicle on the ground level is shown by Eq. (1):

$$
F_{t}=F_{a}+F_{r}
$$

If the vehicle is moving at an inclined level, it can be expressed by Eq. (2): 


$$
F_{t}=F_{a}+F_{r}+F_{g}
$$

The force needed for the linear acceleration of the vehicle is $F_{a}$. The required force to overcome the resistance to vehicle traffic is $F_{r}$. The power required to overcome the effect of gravity is $F_{g}$. The positive signal is used when the vehicle goes up to the sloped level, while the negative signal is used if the vehicle drops to the skewed level.

\subsection{Calculating the needed force for acceleration}

The effect of force motion $F_{a}$ on a vehicle mass of $m \mathrm{~kg}$ and its weight $W$ and the value of the vehicle linear acceleration generated are calculated by Eq. (3):

$$
F_{a}=m w * a
$$

The vehicle contains mechanical rotary parts, such as pedals, front and rear axles, actuators, and gearbox. The equivalent mass $\left(m_{e}\right)$ vehicle rises by $10-20 \%$ from the static mass of the vehicle as clarified by Eq. (4):

$$
F_{a}=m e \cdot a=(W e / g) a
$$

where $m_{e}$ is measured in $\mathrm{Kg}, F_{a}$ is in $\mathrm{N}$, and $a$ is in $\left(\mathrm{m} / \mathrm{s}^{2}\right)$.

\subsection{Calculating the required force to overcome resistance of vehicle}

The force which is opposed the vehicle movement is called mechanical resistance such as friction forces which existed in the axles, gears, and tires at starting. It does not depend on the speed of the vehicle and the mass of the vehicle. Wind resistance increases with the vehicle speed. Assume that $F_{r}$ is the resistance force to the vehicle in ( $\mathrm{N} /$ ton) as shown by Eq. (5).

$$
F_{r}=m \times r
$$

\subsection{Calculating the force needed to overcome the effect of gravity}

If the vehicle goes uphill at an angle $\theta$, then the force $F_{g}$ is resolved to two components and will be affected by the height axis $\operatorname{Sin} \theta$ as expressed by Eq. (6):

$$
F_{g}=W \times \sin (\theta)=m \times g \times \sin (\theta)
$$

If $C$ is the slope percentage is as expressed by Eq. (7), $F_{g}$ is again identified by Eq. (8):

$$
\begin{gathered}
C \%=(Y / X) .100=100 \sin \theta \\
F_{g}=m \cdot g \cdot C
\end{gathered}
$$

\subsection{Total traction force on the tire}

$$
\begin{gathered}
F_{t}=F_{a}+F_{r}+F_{g} \\
F_{t}=m e \times a+m \times r+m \times C
\end{gathered}
$$

$\mathrm{r}$ is measured by $\mathrm{N} / \mathrm{Kg}$. The positive sign is used when the vehicle is upward to the sloped level, while the negative sign is devoted for the vehicle downward. 


\subsection{The driving power for moving the tires}

If the vehicle is roaming at a constant speed $V$ of $\mathrm{m} / \mathrm{s}$, the needed power $P_{o}$ for movement is calculated by Eq. (11):

$$
P_{o}=F_{t} \cdot V
$$

If the gear efficiency is $\eta$, the needed power by electric motor $P_{m}$ is calculated by Eq. (12):

$$
P_{m}=P_{o} / \eta=F_{t} \times V / \eta
$$

\subsection{Mechanical vehicle movement}

The movement of the motor spindle, which makes torque in the rotary tires, is transmitted using a gearbox to reduce the speed from $N_{m}$ to $N$ rpm, if:

The torque induced from the traction force on the tire $T$ The traction force which drives the gears $F_{1}$.

Traction force on the tire $F_{t}$ Motor speed $N_{m}$.

Wheel speed $N$.

Gear ratio $d_{2} / d_{1}=\sigma$.

Diameter gear motor/diameter gear wheel stirring $=d 1, d 2$.

Diameter of tire (tire) $D$.

Gearbox efficiency $\eta$.

The relation between the motor torque $T_{m}$ and traction torque required by the tire $T$, gear conversion ratio, and gearbox efficiency $\eta$ can be simplified through Eqs. (13) to (16):

$$
\begin{gathered}
T_{m}=F_{t} *\left(d_{1} / 2\right) \\
T=F_{t} \times\left(D_{2} / 2\right)=\eta F_{t}(d 2 / 2) \\
F_{t}=F_{1} \eta\left(d_{2} / D\right) \\
F_{t}=F_{1} \eta\left(d_{2} / D\right)=\eta \times 2\left(T_{m} / d_{1}\right) \times\left(d_{2} / D\right)=2 \eta \sigma\left(T_{m}\right)(D)
\end{gathered}
$$

Ft depends on many factors: weight, wind resistance, and gradient. Furthermore, the traction force is also increased with the growing friction factor $\mu$ so that the total traction force (10) can be adjusted to Eq. (17):

$$
F_{t}=\mu \times m_{e} \times a=m \times r+m \times C
$$

\section{Experimental tests and mathematical calculations}

\subsection{Electrical design}

\subsubsection{Motor and vehicle}

The three-phase synchronous motor used (3PSM) is a BLDC-YG1-ZZ- $1200 \mathrm{~W}$ type. The choice of this motor is based on the speed and torque necessary for the load. The 3PSM is provided by an AC three-phase line voltage of 380 volt and $50 \mathrm{~Hz}$. The motor maximum speed is $3000 \mathrm{rpm}$. The torque $T_{m}$ of the motor can be calculated by using Eq. (18). $P_{o}$ is the shaft output power. The angular velocity $\omega$ is explained by Eq. (19), where $N$ is the speed in rpm unit [5]: 


$$
\begin{gathered}
T_{m}=P_{o} / \omega \\
\omega=\frac{2 \pi N}{60}=314.16 r p s
\end{gathered}
$$

where according to the real specification of the 3PSM, the torque of the motor can be calculated using Eqs. (1) and (2):

$$
T_{m}=1200 \mathrm{~W} /(2 \times 3.14 \times 3000 / 60)=3.8 n \cdot m
$$

This anticipated torque is significantly adequate with $3000 \mathrm{rpm}$ for the present load and as an input of mechanical gearbox. The next qualifications of the mechanical gear box will depend firmly upon the two manufactured torques: torque of the 3PSM and traction torque required for the vehicle load. Figure 2 shows the three-phase synchronous motor which is used for vehicle and its nameplate.

\subsubsection{Gearbox and vehicle load}

The gearbox used in the vehicle is shown in Figure 3. It is subjected to many factors: input and output speeds and input and output torques required. The present vehicle has a steady-state linear speed which reaches slightly more than $40 \mathrm{~km} / \mathrm{hr}$. The angular velocity of vehicle tires is explained by Eq. (20), where $r$ is identified as the tire radius. The real tire radius is measured to give $0.125 \mathrm{~m}$. The angular speed of gearbox pulley is determined by Eq. (21):

$$
\begin{gathered}
V_{t}=2 \pi r \omega_{t} \\
\omega_{t} D=\omega_{2} d_{2}
\end{gathered}
$$

where according to the real linear speed of the vehicle' tires, the angular speed of the tire can be calculated using Eq. (3):

$$
\begin{aligned}
& 40000 /(60 \times 60)=2 \pi \times 0.135 \times \omega_{t} \\
& \omega_{t}=13.1 \text { rps }
\end{aligned}
$$

Then, the rotation speed the gearbox output pulley $\omega_{2}$ is calculated using Eq. (4):

$$
\begin{aligned}
& 13.1 \times 0.27=0.04 \omega_{2} \\
& \omega_{2}=88.425 r p s
\end{aligned}
$$

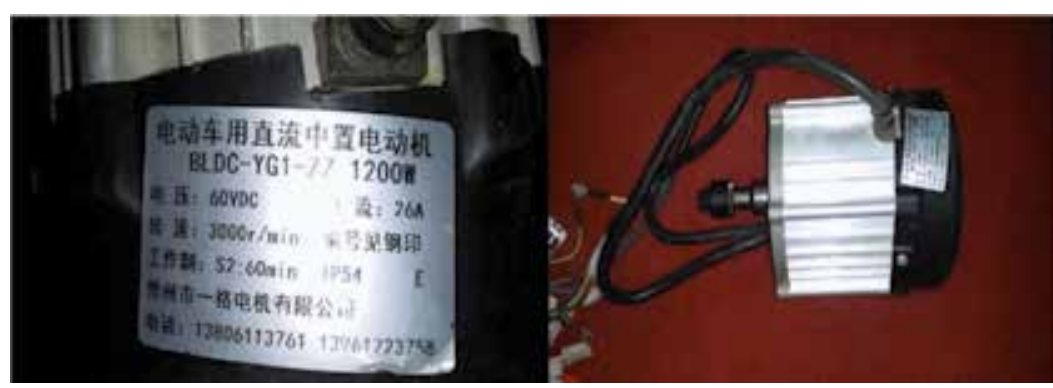

Figure 2.

Three-phase synchronous motor for vehicle traction and its nameplate. 


\subsubsection{DC/AC inverter}

The DC to three-phase inverter is designed as a frequency changer [6]. The synchronous speed of the 3PSM is various using the frequency changer and is determined by Eq. (22):

$$
N_{s}=120 f / p
$$

where from the experimental tests of the 3PSM conditions, the real number of stator slots is designed to give 18 slots. The stator has three phases, and six coils/ phases are determined; for the motor shaft speed of $3000 \mathrm{rpm}$, it means that there are three coils per one pole inside the motor stator. Then, by using Eq. (22) and the obtainable data, the range of input frequency $f$ can be calculated:

$$
3000=120 f / 6
$$

Then the frequency $f$ of $300 \mathrm{~Hz}$ represents the maximum frequency necessary required from the three-phase inverter to the 3PSM. This frequency leads the vehicle and reaches to a maximum linear speed of $40 \mathrm{~km} / \mathrm{h}$.

The real inverter designed is explained by Figure 4 .

\subsubsection{Batteries}

Five series batteries are connected; each battery is a 12 VDC and 14 AH lead-acid sealed type. The entire voltage is $60 \mathrm{VDC}$ which is supplied to the terminal inputs of the inverter. Figure 5 highlights the used battery.

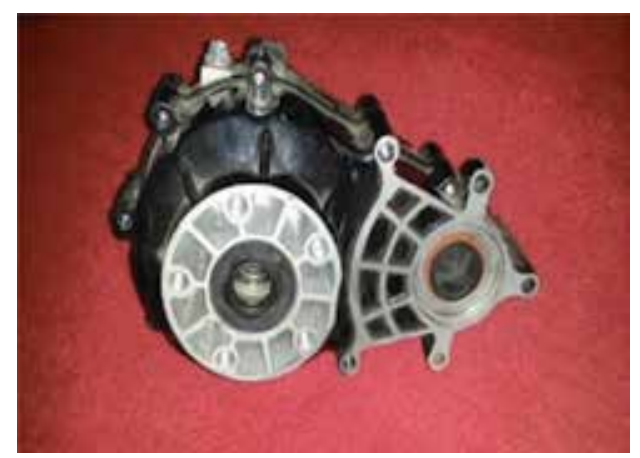

Figure 3.

Motor gearbox.

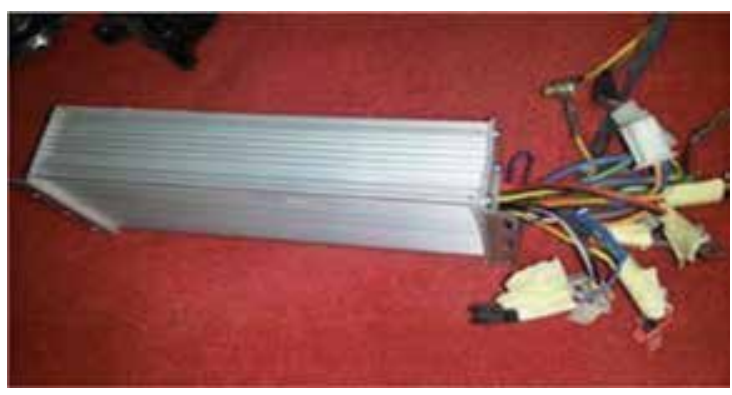

Figure 4 .

The DC to three-phase voltage inverter used. 


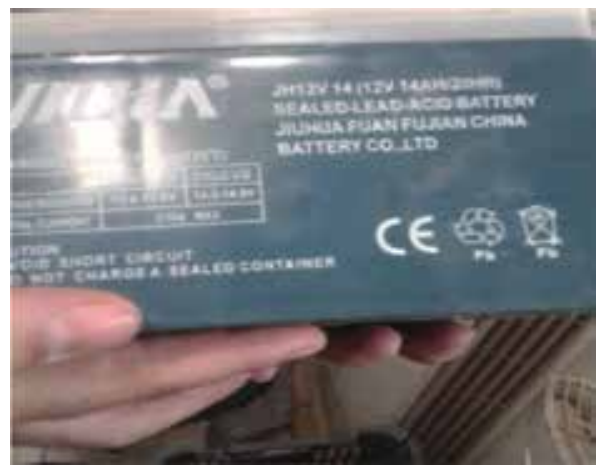

Figure 5 .

The used battery of 12 VDC and 14 amp.hr.
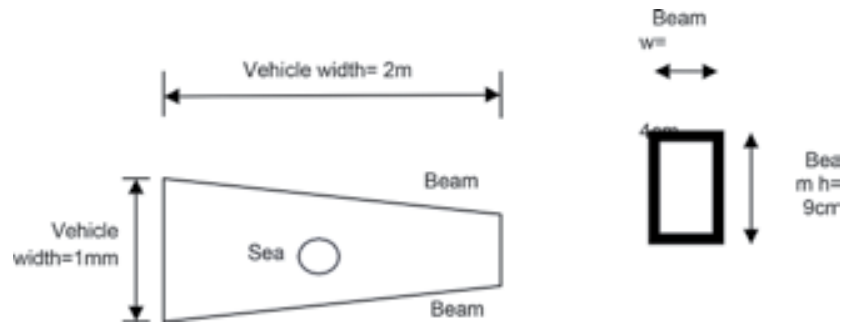

Figure 6.

Dimensions of the vehicle iron beams 1 and 2.

\subsection{Mechanical design}

\subsubsection{Binding stress calculations $\tau_{b}$}

The main supports and joints used for the vehicle frame are iron hollow rectangular metal beams with dimensions of 1.5 inch and thickness of $2 \mathrm{~mm}$. The length of the iron beams 1 and 2 is the same; each beam length is $2 \mathrm{~m}$. Figure 6 shows the lengths of the vehicle structure iron beams 1 and 2 .

The bending stress is determined at the center of beam 1 and beam 2 . The total weight of the driver, five batteries, and solar panel are checked to give a weight of $125 \mathrm{~kg}$. The gravitation force downward by total weight is calculated using Eq. (23):

$$
\begin{gathered}
F_{\omega}=m_{\omega} \times a_{g} \\
F_{\omega}=125 \times 9.8=1225 \mathrm{~N}
\end{gathered}
$$

The bending stress will be determined using Eq. (24):

$$
\tau_{b}=F_{b} / A_{b}
$$

The bending force is distributed into beams 1 and 2 at the middle of each, so the force $F_{w}$ must be divided by 4 . The length of beams is $2 \mathrm{~m}$ as shown in Figure 6 , and the rectangular dimension length of cross-sectional area of beams 1 and 2 is $9 \mathrm{~cm} \times 4 \mathrm{~cm}$, and its thickness is $1.5 \mathrm{~mm}$. The $\tau_{b}$ calculation is implemented using Eq. (24) [3]: 


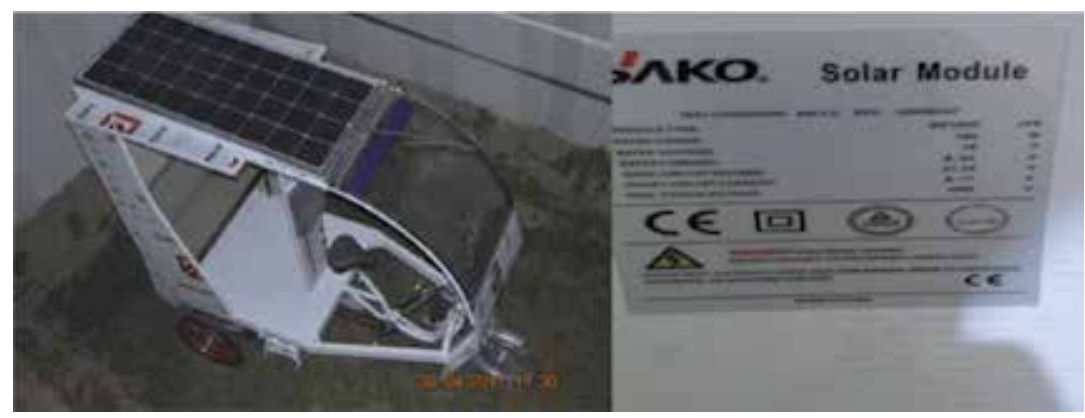

Figure 7.

Solar panel is supplying power to the batteries.

$$
\begin{aligned}
\tau_{b} & =\frac{\left(F_{b} / 4\right)}{(2(h+\omega) \times t)}=\frac{(1225 / 4)}{(2(0.09+0.04) \times 0.015)} \\
& =78.5 \frac{\mathrm{N}}{\mathrm{m}^{2}}<<\text { Bending stress of mild iron } 360 \frac{\mathrm{MN}}{\mathrm{m}^{2}}
\end{aligned}
$$

where $A_{b}$ is the cross-sectional area of hollow rectangular beams 1 and 2 at the tires' shafts.

\subsubsection{Axial shear stress calculations of each tire $\tau$ s}

The total axial force $F_{s}$ (similar to $F_{w}$ ) is composed by the driver, five batteries, and the iron frame weights which it is concentrated onto three tires (two rear tires and one in front). The entire force $F_{s}$ should be divided by three tires and two rod sides per tire. The three circular iron solid rods have each 1 centimeter diameter. The axial shear stress $\tau_{s}$ can be determined using Eq. (25):

$$
\begin{gathered}
\tau_{s}=F_{s} / A_{s} \\
\left.\tau_{s}=F_{s} /\left(3 \text { tires } \times 2 \text { sides } \times A_{r}\right)=(1225) /(3 * 2 * 3.14 *(0.01) 2 / 4) * 0.015\right) \\
=2.6 \mathrm{MN} / \mathrm{m}^{2}[1]<<\text { shear stress of mild iron } 36 \mathrm{MN} / \mathrm{m}^{2} .
\end{gathered}
$$

\subsubsection{Pedal and gears}

Two gear discs are used to transfer the mechanical power created by legs for turning the tires and then the vehicle. It is instituted experimentally; the torque established $T_{d}$ by the legs is sufficient to move vehicle which is closed to bicycle. To calculate the torque established $T_{d}$, can be applied by (13) on the pedal and tire system.

$$
T_{d}=125 \mathrm{~kg} \times 9.8 \mathrm{~N} / \mathrm{m}^{2} \times(0.27 / 2)=165.375 \mathrm{~N} \cdot \mathrm{m},
$$

The minimum mechanical torque required to move the vehicle which is less than the torque required for moving the bicycle with the driver is equal to $85 \mathrm{~kg} \times 9.8 \mathrm{~N} / \mathrm{m}^{2} \times(1 / 2)=415 \mathrm{~N} \cdot \mathrm{m}$.

\subsubsection{Solar design}

The solar panel (type SK150D, 150 watts, $18 \mathrm{~V}, 8.33 \mathrm{Amp}$ ) used is considered as additional power source and has the following specifications as shown in Figure 7 through its nameplate. The rated power produced is amplified by using power 
electronic chopper (low DCV/high DCV) to convert the low-DC volt input to the required volt which is $60 \mathrm{DCV}$. The solar panel is used for charging the five batteries under the sunlight.

\section{Results and discussion}

The investigational tests on the electric motor of the vehicle are shown for the three different speeds, the AC line-to-line voltages are measured, and instantly the current is drawn by the electric lines. The investigational tests are revealed in Table 2.

Based on these experimental tests, the power input and output of the 3PSM can be calculated using the following performance equations for the 3PSM [3]:

$$
\begin{aligned}
& P_{\text {in }}=1.73 V_{L} I_{L} \cos \theta \\
& P_{m o}=T_{s h} \omega=\eta_{m} P_{\text {in }}
\end{aligned}
$$

The results of test 1 are shown in Table 2. By using Eq. (26), the power factor can be supposed as $0.8[5,7]$ :

$$
P_{\text {in }}=1.73 \times 36 \times 5.13 \times 0.8=255.6 \mathrm{~W}
$$

Referring to test 2, $P_{\text {in }}=363.6 \mathrm{~W}$. Pin $=456.72 \mathrm{~W}$ for test 3 .

Then, efficiency of the 3PSM can be supposed as $0.9[5,7]$. By reusing Eqs. (20), (21), and (27), the torque induced is:

$$
0.9 \times 456.72=2 \pi \times(1500 / 60) \times T_{s h}
$$

$T_{s h}=2.6 \mathrm{~N} . \mathrm{m}$ is produced by the motor initially to increase it through the gearbox. Finally the required torque is achieved for moving the vehicle.

Test no. Vehicle speed ( $\mathrm{km} / \mathrm{hr}) \quad$ 3PSM line-to-line voltage (volt) 3PSM line current (ampere)

\begin{tabular}{cccc}
\hline 1 & 20 & 36 & 5.13 \\
\hline 2 & 30 & 47 & 5.59 \\
\hline 3 & 40 & 55 & 6 \\
\hline
\end{tabular}

Table 2.

Experimental tests.

\section{Conclusion}

The investigational tests and theoretical calculations of the vehicle show that there is a good confirming result which satisfied the required torque and speed. There is some enhancement that needs to be applied in the future to reduce the iron size of the two beams by using a smaller size. Furthermore to that, it is possible to use four tires instead of three tires. Also electronic control devices can be advanced by using Arduino controller applications. 


\section{Author details}

Khalid G. Mohammed

Scientific Affairs Department, University of Diyala, Iraq

*Address all correspondence to: khalid_alkaisee2013@yahoo.com;

khalid_alkaisee@engineering.uodiyala.edu.iq

\section{IntechOpen}

(C) 2020 The Author(s). Licensee IntechOpen. This chapter is distributed under the terms of the Creative Commons Attribution License (http://creativecommons.org/licenses/ by/3.0), which permits unrestricted use, distribution, and reproduction in any medium, provided the original work is properly cited. (c) BY 


\section{References}

[1] Mohammed KG. Experimental investigations on hybrid vehicle. International Journal of Engineering \& Technology. 2018;7(3.17):85-89

[2] Mapelli FL, Tarsitano D, Mauri M. Plug-in hybrid electric vehicle: Modeling, prototype realization, and inverter losses reduction analysis. IEEE Transactions on Industrial Electronics. 2010;57(2):598-607

[3] Schaltz E. Design of a Fuel Cell Hybrid Electric Vehicle Drive System. Department of Energy Technology, Aalborg University; 2010

[4] Ehsani M, Gao Y, Gay SE, Emadi A. Modern Electric, Hybrid Electric, and Fuel Cell Vehicles - Fundamentals, Theory, and Design. first ed. CRC Press LLC; 2005

[5] Emadi A. Handbook of Automotive Power Electronics and Motor Drives. first ed. Taylor \& Francis; 2005

[6] Grag GC. Utilization of Electric Power \& Electric Traction. Kanna Publishers Delhi; 1988

[7] Theraja BL. Textbook of Electrical Technology. Vol. 2. 2010

[8] Subrahmanyam V. Electric Drives: Concepts and Applications. Mc GrawHill; 1990

[9] Sawhney AK. A Course in Electrical Machine Design. Nai Sarak: Dhanpat Rai and Sons; 1984

[10] Hamdi ES. Design Small Electrical Machines. Chichester, UK: John Wiley and Sons; 1994

[11] Available from: https://ar.wikipedia. org/wiki/

[12] Available from: www.ehcar.net/lib rary/rapport/rapport074 


\title{
Torque Control of a DC Motor with a State Space Estimator and Kalman Filter Applied in Electrical Vehicles
}

\author{
Alex Archela, Dario Guilherme Toginho \\ and Leonimer Flávio de Melo
}

\begin{abstract}
This work presents a study over a torque-generated speed control of free wheel attached to a DC motor, for use on traction of mobile vehicles. Also, it presents the discrete state space model of a DC model and the Kalman filter's equations and applications. This work presents a hardware-in-the-loop (HIL) system for design of a torque controller noticed that this process produces a faster design, coding, and parameter optimization of any embedded systems. The hardware used for the implementation of the system is discussed as the hardware-in-the-loop environment which makes possible the fast tuning and design of the system. In the absence of a torque sensor, this work uses the Kalman filter's estimated states torque and speed as feedbacks of the system.
\end{abstract}

Keywords: hardware-in-the-loop, Kalman filter, discrete state estimation, DC motor, closed-loop control, electrical vehicles, torque control, embedded control systems

\section{Introduction}

More attention has been taken on electrical vehicles for a series of motives, like price of oil increasing and concern about global environmental problems [1]. The study on this area has been searching for methods of increasing the energy efficiency, safety, stability, and performance of those systems [2, 3].

Another aim of the researchers is to develop efficient control strategies for navigation, but without an accurate control of the traction motors, the trajectory desired cannot be followed [4]. Traction control of vehicles can be very tricky though, because the friction coefficient between the tire and surface is nonlinear and uncertain [5]. This effect also happens for applications in mobile robots with torque higher than the surface friction; hence, the velocity control cannot assure that the trajectory will be followed.

The architecture of four independent motors actuating on each wheel is presented by $[5,6]$. Usually, each wheel is controlled by a torque controller, direct or indirectly, as in [7], which proposes an optimum torque distribution strategy of 
four independent motors on an electric vehicle, considering its driving force and yaw moment ratios testing on slippery road conditions.

As pointed by [8], the torque of an electric motor can be generated quickly and accurately and also can be easily measured through an observer, making it possible to create a drive force observer. This work presents a study over the torque control of a DC motor, which the electromagnetic torque is estimated, with a discrete state space estimator and a Kalman filter [9].

\section{System design}

In this section, the mechanical system is described, which is the aim of the control study; it also presented the mathematical description of this model, so it can further be applied in this work.

\subsection{Mechanical system}

The choice of physical implementation for testing, setting, and tuning is a motor-wheel system, where a motor is attached to a suspended wheel with the use of a crown, pinion, and chain. This system provides an actuation of the motor on acceleration and braking of the wheel, making it possible to generate a full cycle of control. Figure 1 presents the current system.

Notice that the motor is attached directly to the wheel instead of an axis, providing the possibility of a full cycle of control of the wheel speed and torque by the motor.

\subsection{Dynamic model}

The approach of the independent-driven wheels for vehicle traction results in a DC motor attached to a single wheel. Therefore, knowingly Eqs. (1)-(3) describe the electric DC motor states:

$$
v(t)=R_{a} i_{a}(t)+L_{a} i_{a}{ }_{a}+e(t)
$$

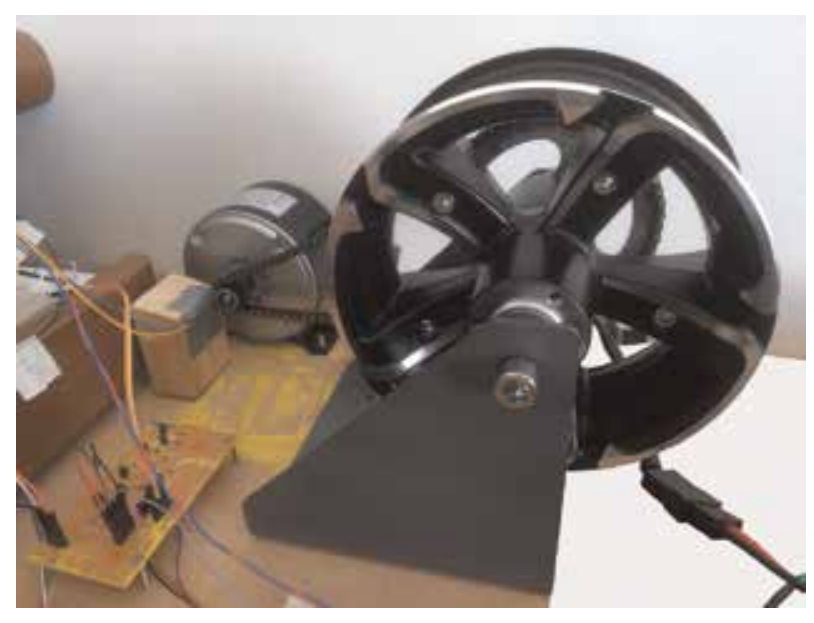

Figure 1.

Suspended wheel attached to the traction motor by a crown-chain-pinion. 
Torque Control of a DC Motor with a State Space Estimator and Kalman Filter Applied... DOI: http://dx.doi.org/10.5772/intechopen.87306

$$
\begin{aligned}
e(t) & =K_{e} \omega(t) \\
T_{m} & =K_{t} i_{a}(t)
\end{aligned}
$$

where $v(t)$ is the voltage supplied to the motor $(\mathrm{V}), R_{a}$ the armature resistance $(\Omega), i_{a}$ the value of armature current $(\mathrm{A}), L_{a}$ the armature inductance $(\mathrm{H}), e(t)$ the back electromotive force $(\mathrm{V}), K_{e}$ the back electromotive constant (V.s/rad), $\omega(t)$ the motor shaft speed ( $\mathrm{rad} / \mathrm{s}), T_{m}$ the motor electromagnetic torque (N.m) and $K_{t}$ is the motor torque constant (N.m.A). Substituting (2) and (3) in (1) yields (4):

$$
v(t)=R_{a} i_{a}(t)+L_{a} i^{\prime}{ }_{a}+e(t)
$$

Apart from the DC motor, the single wheel attached to the motor alters the dynamic equations for the system. Applying Newton's second law of motion on the rotational movement results in Eq. (5):

$$
J_{T} \frac{d \omega(t)}{d t}=T_{m}(t)-T_{L}(t)-B_{T} \omega(t)
$$

Given that $B_{T}$ is the coefficient of viscosity $\left(\mathrm{Kgm}^{2} / \mathrm{s}\right)$ of the system and $J_{T}$ is the moment of inertia $\left(\mathrm{Kgm}^{2}\right)$ generated by the sum of the motor shaft $\left(J_{m}\right)$, the wheel $\left(J_{r}\right)$ is given by Eq. (6):

$$
e(t)=K_{e} \omega(t)
$$

Considering $\omega(\mathrm{t})$ and $T_{m}(\mathrm{t})$ the system states and $T_{L}(\mathrm{t})$ is zero, it is possible to obtain the state space matrices for the DC motor, given Eqs. (4) and (5).

\subsection{State space of a DC motor}

Selecting the states previously elected and knowing that $\mathrm{v}(\mathrm{t})$ and $T_{L}(\mathrm{t})$ are inputs of the system, Eqs. (4) and (5) can be rewritten as (7) and (8):

$$
\begin{aligned}
& \dot{x}=A x+B u \\
& y=C x+D u
\end{aligned}
$$

resulting in (9) and (10)

$$
\begin{gathered}
{\left[\begin{array}{c}
\dot{\omega}(t) \\
\dot{T_{m}}(t)
\end{array}\right]=\left[\begin{array}{cc}
\frac{-B_{m}}{J_{m}} & \frac{1}{J_{m}} \\
\frac{-K_{e} K_{t}}{L_{a}} & \frac{-R_{a}}{L_{a}}
\end{array}\right]\left[\begin{array}{r}
\omega(t) \\
T_{m}(t)
\end{array}\right]+\left[\begin{array}{lr}
0 & -1 / J_{m} \\
K_{t} / L_{a} & 0
\end{array}\right]\left[\begin{array}{r}
v(t) \\
T_{L}(t)
\end{array}\right]} \\
R=E\left[\nu \nu^{T}\right] .
\end{gathered}
$$

Therefore, the output of the system given by Eq. (10) is only the current rotor speed, once there is no torque sensor integrated on the system. Also the matrix D is equal to zero, because there is no interference on the input of the system directly to the output.

Although the state space obtained describes the motor inputs and outputs for a continuous time application, these matrices cannot be applied on a digital discrete 
system. Hence, for an implementation of the digital control, it is needed to obtain the state space discrete model described by (9) and (10) with a sample period of $\tau$. For that, (7) becomes (11):

$$
P[k]=\Phi \tilde{P}[k-1] \Phi^{T}+Q
$$

where

$$
T_{\mathrm{m}}=K_{\mathrm{l}} i_{\mathrm{a}}(t)
$$

and

$$
J_{T} \frac{d \omega(t)}{d t}=T_{m}(t)-T_{L}(t)-B_{T} \omega(t)
$$

\section{System implementation}

In this section, the hardware used for the development of this work as the introduction of the hardware-in-the-loop applied system is described. Also, the motor and mechanical parameters for tuning the controllers are presented.

\subsection{System description}

The hardware-in-the-loop (HIL) process is composed of the real physical system, to be controlled, activated by an embedded microcontroller which communicates directly to the virtual environment of design in the computer, as illustrated in Figure 2. The embedded controller is made through the BeagleBone Black, programed through the software MATLAB/Simulink, allowing fast prototyping and tuning of the controller settings and observation of the results.

As illustrated in Figure 2, the controller design is made through MATLAB/ Simulink, where it is also possible to tune the controller parameters and observation of the results in real time, without compromising the experiment execution. The results can be observed through real-time graphs that can also be stored for later analysis.

The controller design is created through Simulink block diagram that automatically generates a code compatible to the microcontroller and downloads it to the embedded controller system. The embedded system, on the other hand, is dedicated to execution of the motor control, estimating states and sensors reading.

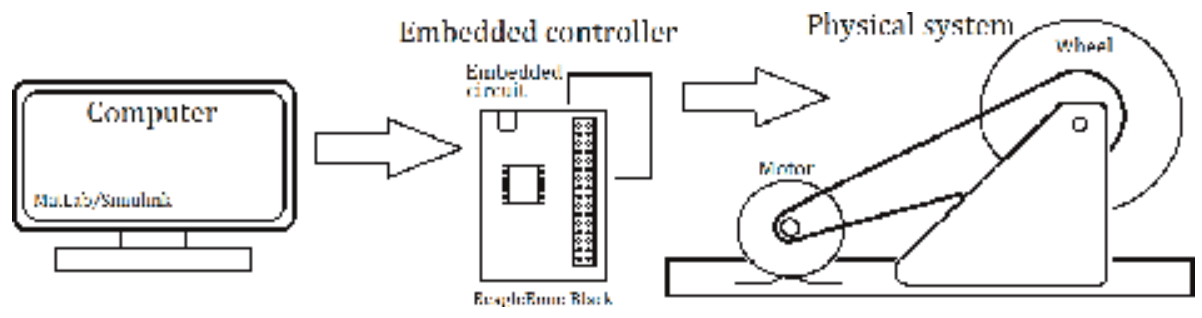

Figure 2.

Illustration of hardware-in-the-loop system. 
Torque Control of a DC Motor with a State Space Estimator and Kalman Filter Applied... DOI: http://dx.doi.org/10.5772/intechopen.87306

\begin{tabular}{lccccc}
\hline $\mathrm{Ra}[\mathbf{\Omega}]$ & $\mathrm{La}[\boldsymbol{\mu H}]$ & $\mathrm{Ke}[\mathrm{Vs} / \mathbf{r a d}]$ & $\mathrm{Kt}[\mathrm{Nmrad} / \mathbf{s}]$ & $\mathrm{Bm}\left[\mathrm{Kgm}^{2} / \mathbf{s}\right]$ & $\mathrm{Jm}\left[\mathrm{Kgm}^{2}\right]$ \\
\hline 4.735 & 344.6 & 0.0236 & 0.0424 & 0.15379 & 0.0005 \\
\hline
\end{tabular}

Table 1.

Parameters of the mechanical system.

\subsection{Hardware}

A DC motor model MY1016 is used for the traction of the wheel for the studied system, which has a nominal voltage of $24 \mathrm{~V}$, current of $13.7 \mathrm{~A}$, maximum velocity of $2650 \mathrm{RPM}$, and nominal power of $250 \mathrm{~W}$. For the system power supply, two batteries of $12 \mathrm{~V}$ and 7.0 Ah are used, powering the motor driver.

The motor velocity is obtained through a Hall effect sensor u1881 and six pairs of magnets attached to the motor shaft with alternated polarities, so when the motor spins, it produces six rising and falling edges per revolution.

For the crown-chain-pinion set, a 11 teeth pinion on the motor axis is used, while the wheel has a 55 teeth crown, generating a mechanical advantage of 5 , meaning the motor axis has a rotational speed five times faster than the wheel; meanwhile, its torque is five times higher.

With a diameter of $18.0 \mathrm{~cm}$ in the wheel, the maximum speed expected by the system is $17.98 \mathrm{~km} / \mathrm{h}$, resulting in a system speed high enough for the analysis used in this work. The embedded system is controlled by a BeagleBone Black ARM Cortex-A8 microcontroller, which has up to $1 \mathrm{GHz}$ of frequency. The parameters that describe the state space for the torque control are provided in Table 1.

\section{Hardware-in-the-loop}

This section describes hardware-in-the-loop process used in this work; it also presents the configuration and workflow of the current system.

\subsection{MATLAB/Simulink embedded coder}

The system in hardware-in-the-loop allows the fast prototyping of a control system, allowing the observation of speed, control signal graphs, and observation of states in real time, provided by the embedded board BeagleBone Black. It also provides a result much more closer to reality than the pure simulation [10].

Once the coding does not need to be tested in a complete real situation, the presence of HIL system turns out to be a low-cost solution for engineering problems [11]. Allowing the code to be directly generated and optimized by the embedded coder from the Simulink environment [12].

This processor is responsible for sensor reading and processing the actual speed, given the sensor signals provided, as estimation of the current torque value. Besides, the PWM signals controls the motor through its driver. Figure 3 illustrates the HIL system workflow.

As shown in Figure 3, the design of the project is previously created in Simulink virtual environment of design. Through the use of code generator provided by MATLAB, the environment generates a compatible code to the embedded controller and downloads the code.

Once the code is downloaded, the embedded system still maintains a communication in background with the physical computer but, in second plane, without compromising the current experiment. This communication provides the value of 


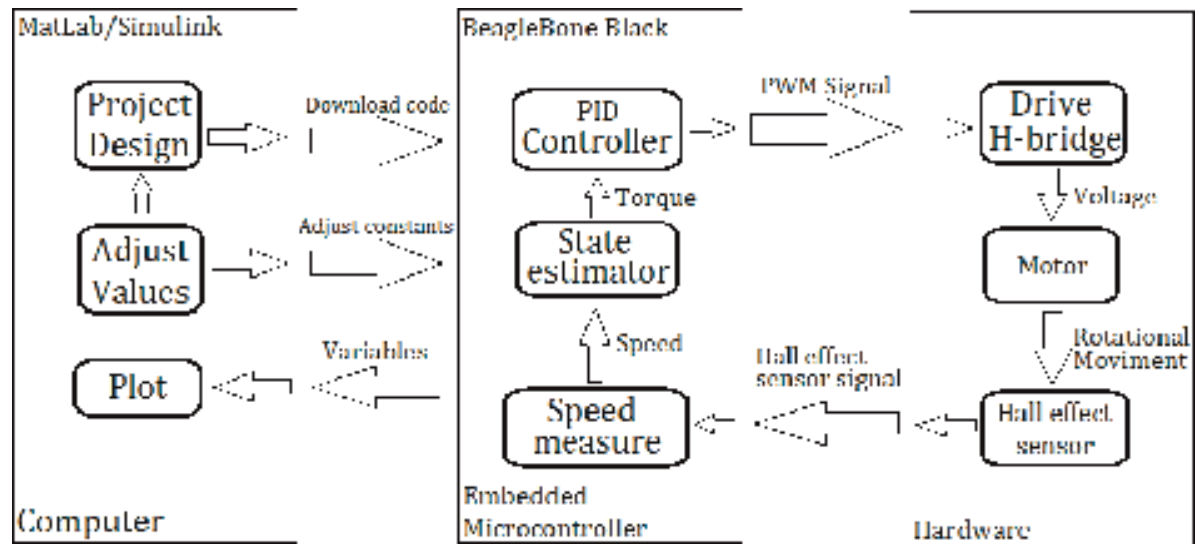

Figure 3.

Schematic of a hardware-in-the-loop system design.

specific variables, so the results can be observed in real time. Also, it is possible that new adjusts in variables inside the controller designed can be transferred to the embedded controlled in the current experiment, without the necessity of terminating the simulation and starting again.

The embedded board is responsible for all the execution of the digital controller; hence, the speed sensor is an input to the microcontroller, which processes the information and estimates the respective speed; this speed works as an input to the Kalman filter that works not just as an estimator for the torque but also filters the noise generated by speed sensor. By adding a Kalman filter, the vibration of the system is reduced, creating a much more efficient controller [13]. As a result, the current torque works as an input for the feedback of a PID controller, producing PWM signals for the motor drive.

For the physical system, the input is the PWM signals that provide not just the information of the amplitude in which the motor will be activated but also its direction, depending on the current PWM activated. This signal activates the interface of activation of the motor producing the correction on the motor torque and speed. As the motor axis rotational speed produces a rotational movement on the motor axis, the Hall effect speed sensor produces a signal that works as an output, providing a feedback for closed-loop control system [14].

\subsection{MATLAB/Simulink configuration}

For the implementation of the HIL system, it is needed to follow some configuration steps. The first step is to assure that MATLAB has support for the desired embedded board; in this present research, it was chosen to use the BeagleBone Black board. The support packages can be found in Add-Ons $\rightarrow$ Get Hardware Support Packages.

Those which are necessary to successfully communicate with MATLAB are ARM Cortex-A: Generate code optimized for ARM Cortex-A processor, providing the possibility for MATLAB to generate code compatible to an ARM processor Cortex-A.

BeagleBone Black development board interact with MATLAB which in turn generates an optimized code necessary for HIL implamentation.

After the download and installation of each package, it is needed to connect the board to MATLAB and open Simulink configurations in model configuration parameters to properly set the communication with the board, as shown in Figure 4. 
Torque Control of a DC Motor with a State Space Estimator and Kalman Filter Applied... DOI: http://dx.doi.org/10.5772/intechopen.87306

The configuration parameters are set automatically once the previous installations are successfully done; however, for high demanding control system in HIL which are time dependent, it is necessary to run the communication in the second plane. To configure the HIL in the background, it is necessary to check the option Run external mode in a background thread in the External mode menu.

Another parameter configuration menu panel that is very important for a successfully code generation is depicted in Figure 5.

Language $\mathrm{C}$ and the system target file, ert.tlc, are necessarily set for a successful download of the designed control system, so the code can be understood by the embedded board. This menu also allows the generation of the code for external applications without running in HIL.

For the application of the HIL, after the proper design of the controller has been set, it is necessary to change the mode of execution from normal to external, as

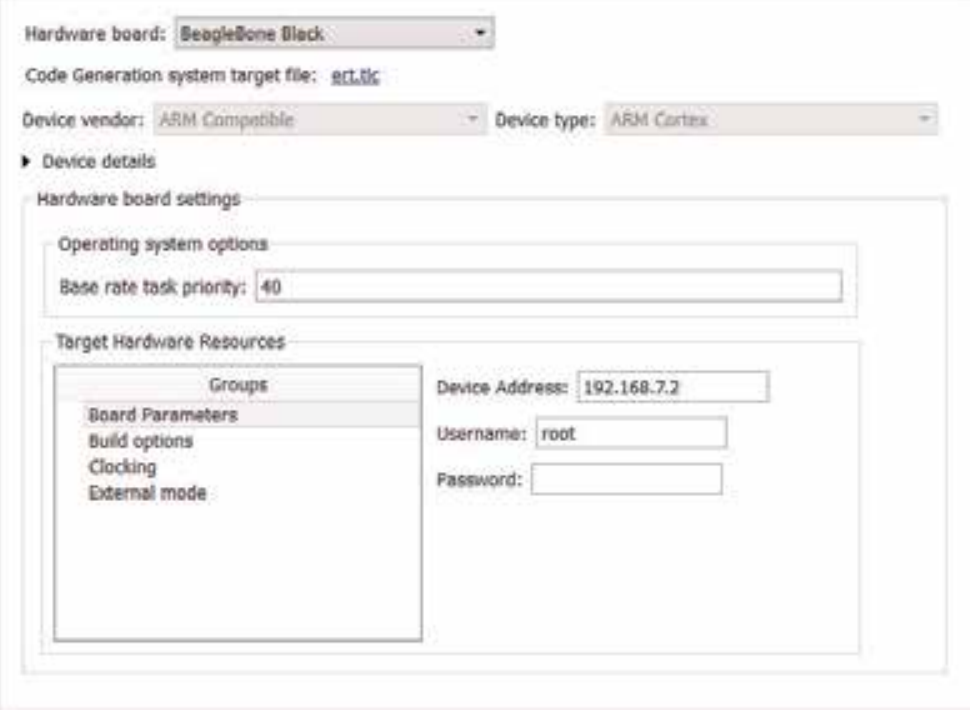

Figure 4 .

Interface of parameter configuration for the hardware communication.

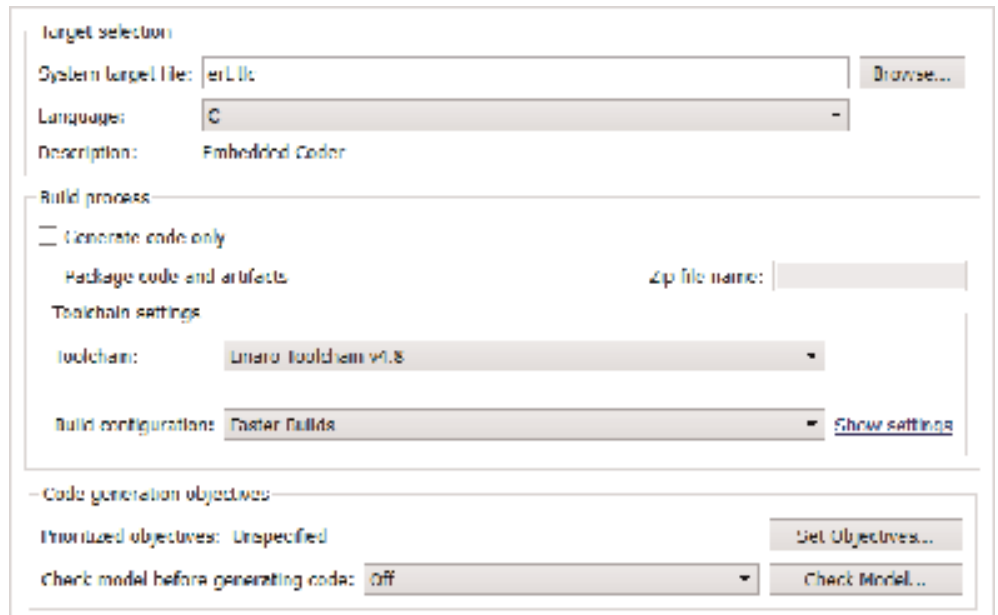

Figure 5.

Interface of parameter configuration for the code generator. 


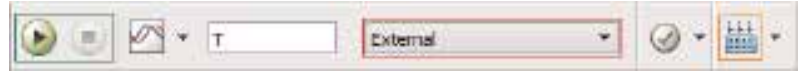

Figure 6.

Illustration for running the code in HIL.

shown in Figure 6, and run the simulation button, instead of deploying on the hardware. While the code is running, Simulink will automatically generate a code and print a report of the code generated and also automatically downloads the code into the embedded board and starts the experiment.

\section{Controller design}

The torque controller as its design as a Simulink block diagram and also the torque-generated speed controller are described in this section, where it also presented its design for application.

\subsection{Torque controller}

Motor's speed could present a high slipping ratio and some times lost of traction, in extreme situations, once the controller has no information about traction and real acceleration, which might be dangerous.

Figure 7 shows a design of a torque controller for the motor, in which the reference is an input for the system. The sensor signal is then read and processed and assigned as one of the Kalman filter inputs, aside from the control signal. The Kalman filter is responsible for filtering noises from the sensor signal and also estimates the current torque, since there is no torque sensor attached to the system.

As a torque control, the output of the Kalman filter which estimates the real current speed is ignored, and the current torque is assigned as the feedback for the controller. The resulting error signal is the input of the discrete PID controller, resulting on a control signal.

This control signal is a feedback to the Kalman filter and also it is normalized, and after passing through a block of separation and generation of the protected PWM signals, the system outputs both PWM signals as generated.

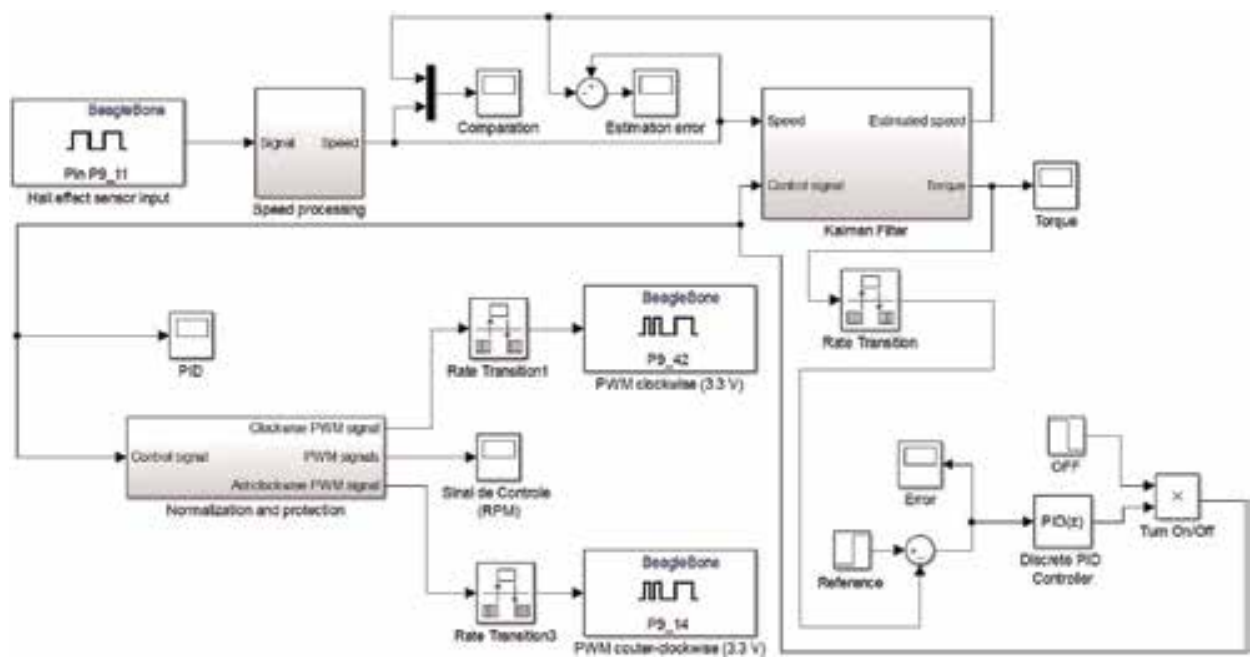

Figure 7.

Torque controller design. 


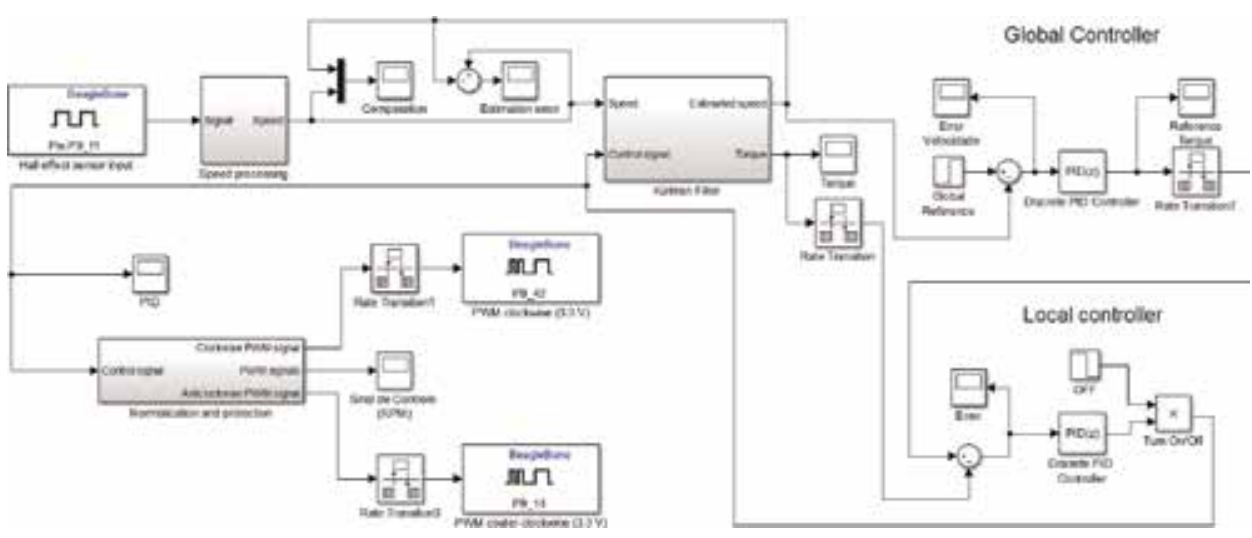

Figure 8.

Torque-generated speed controller design.

\subsection{Torque-generated speed controller}

A second option for the solution of the pure speed controller problems is the application of a torque-generated speed controller, where the controller sets the speed as desired, but it is tuned by a torque controller. This method keeps the torque monitored and can be easily limited and used for noticing traction of the wheel.

The significant difference from the torque controller to the design of the present controller is that the estimated speed is not ignored any longer, but its value is used as a feedback of a global controller, where the speed is controlled by another discrete PID controller.

The resulting signal of this global controller is a torque reference for the previous controller, called local controller. This system then provides a stable speed controller with a monitored torque that provides more safety for the system (Figure 8).

\section{Results}

The results of this work are presented and discussed in this section. Each result is presented with graphs.

\subsection{Torque controller}

The torque controller for a free wheel has low stability, as higher the speed lowers the current torque, but the tuning of a good torque controller allows the increase of stability for other types of controllers.

It is noticeable in Figure 9 that the torque controller manages to stabilize around 0.15 and $0.02 \mathrm{Nm}$, in different states of time, which happens because of the control correction.

The controller actuation can be seen in Figure 10, where the fast response of the controller to correct the stability region in a small period of time is noticeable.

Also it is possible to observe in Figure $\mathbf{1 1}$ the error signal, where it notices the correction of the controller with the time, and visualize the challenge of stability of a torque control in the absence of traction. 


\subsection{Torque-generated speed controller}

Once the pure torque control proves to be challenging, the proposition of developing a torque-generated speed controller seems to produce better results adding the best characteristics of each controller. Figure 12 shows the signal reference of the local controller generated by the global controller and notices the stability of the current system.

As the response to the reference signal, the local controller alters the torquegenerated speed controller which controls the final speed. Figure 13 shows the current torque of the system during the experiment, and it is possible to notice not only the stability of the controller but also an oscillation of the state resultant from the chain that attaches the motor and wheel.

The variation of the resultant torque is caused by the control signal; this signal activates the motor through a driver using two PWM signals that varies from 0 to 1 , and each one controls the direction of the motor activation. Figure 14 shows the PWM signals and its values.

It can be noticed that, periodically, the controller generates a reverse pulse of PWM, caused by the mechanical system chain oscillation, but the controller proves to be stable as the result can be seen in Figure 15.

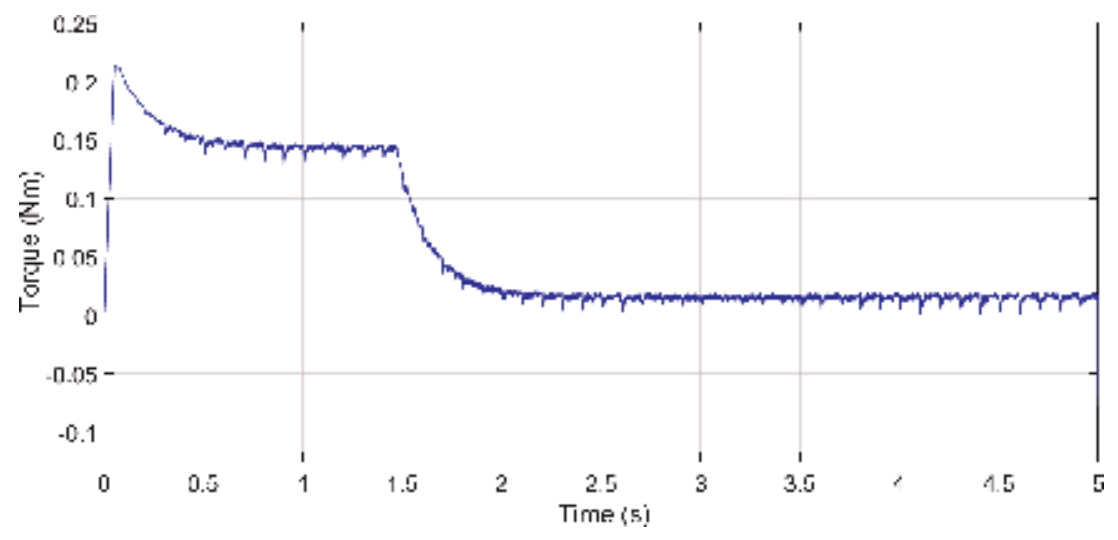

Figure 9.

Torque generated.

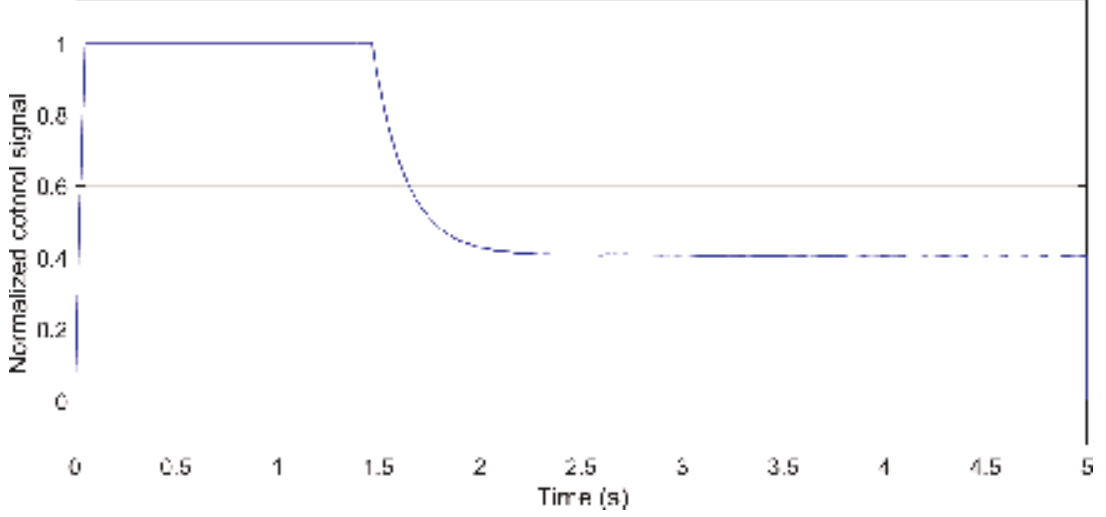

Figure 10.

Normalized control signal. 
Torque Control of a DC Motor with a State Space Estimator and Kalman Filter Applied... DOI: http://dx.doi.org/10.5772/intechopen.87306

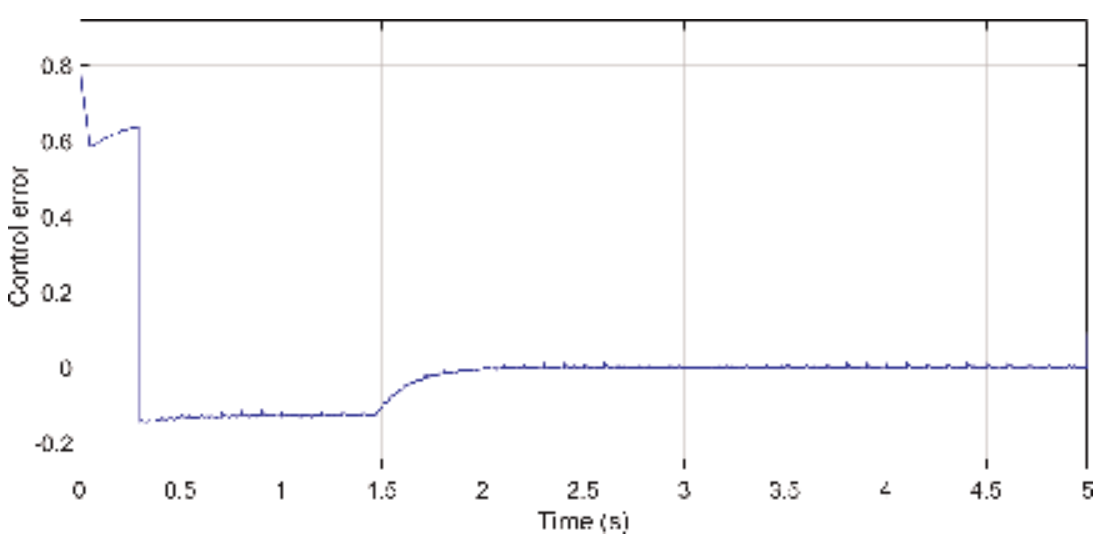

Figure 11.

Torque controller error.

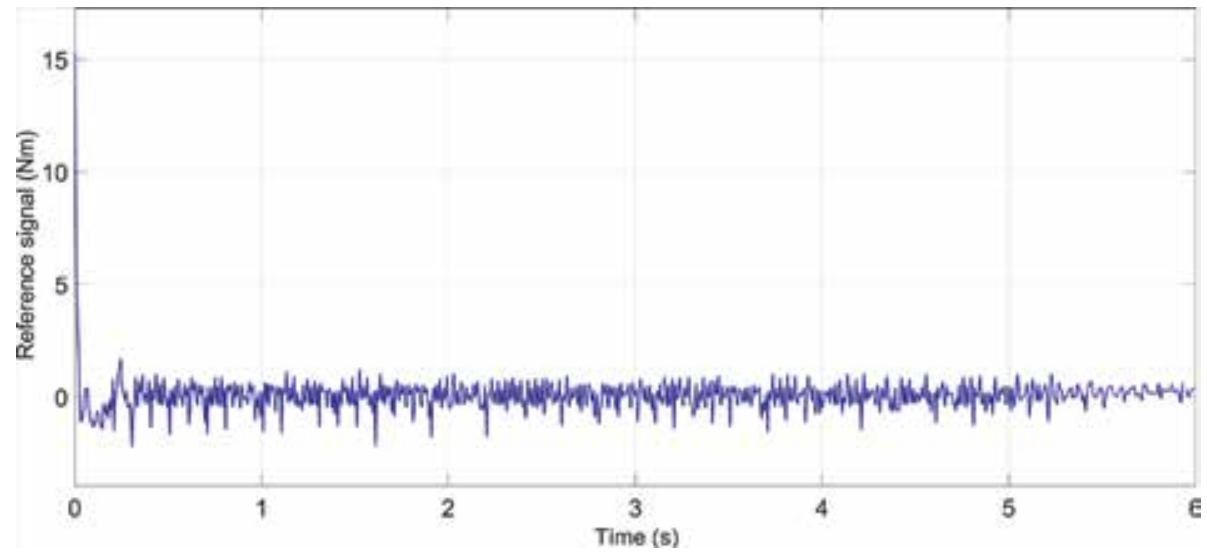

Figure 12.

Reference signal.

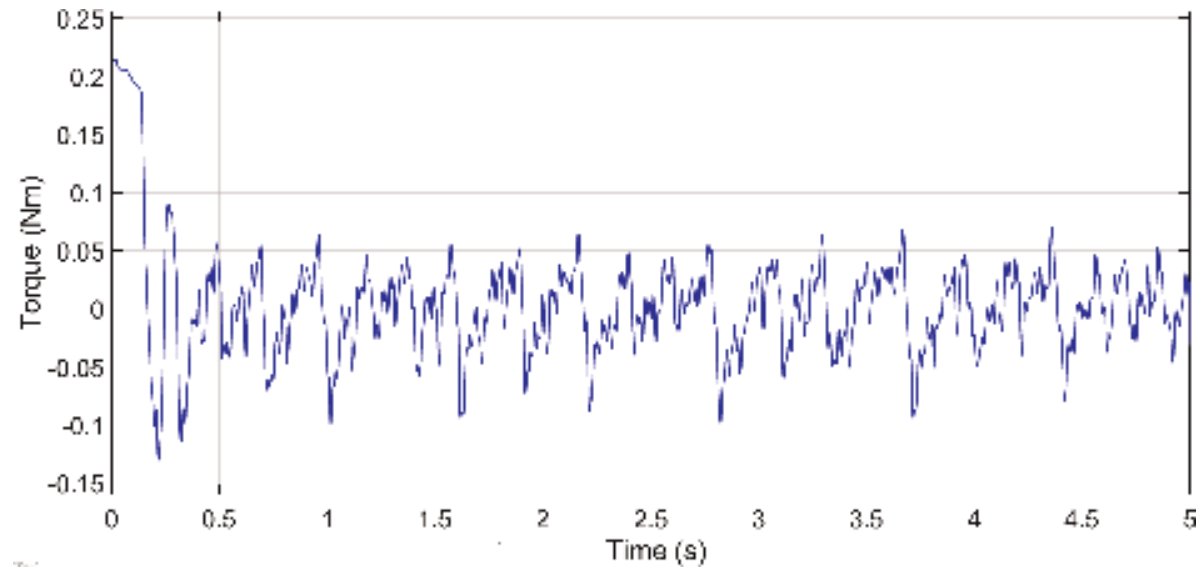

Figure 13.

Torque. 


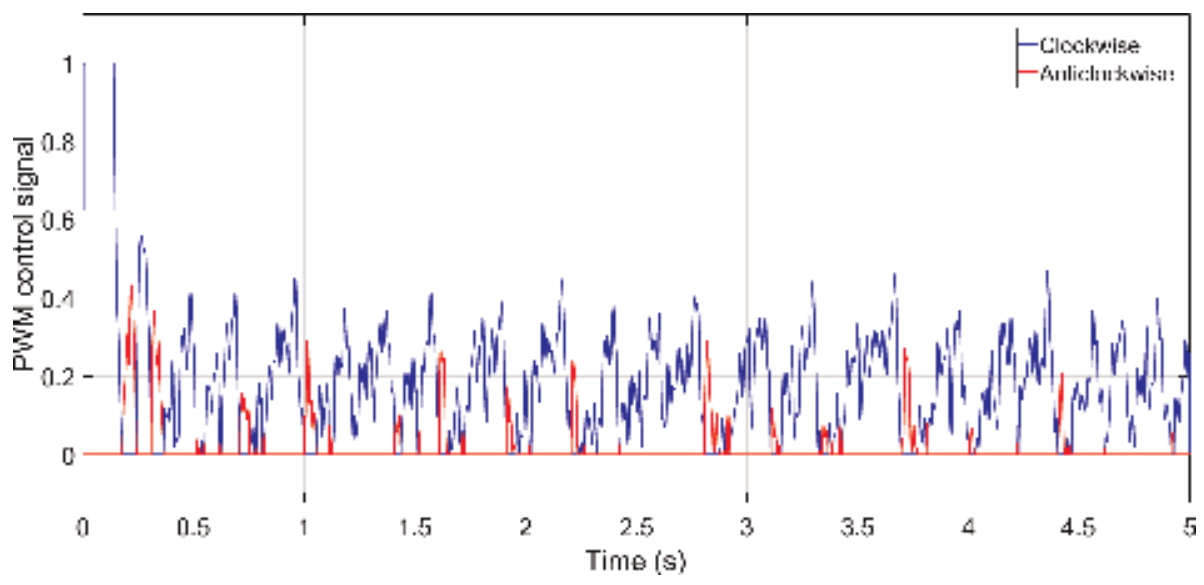

Figure 14.

Control PWM signal.

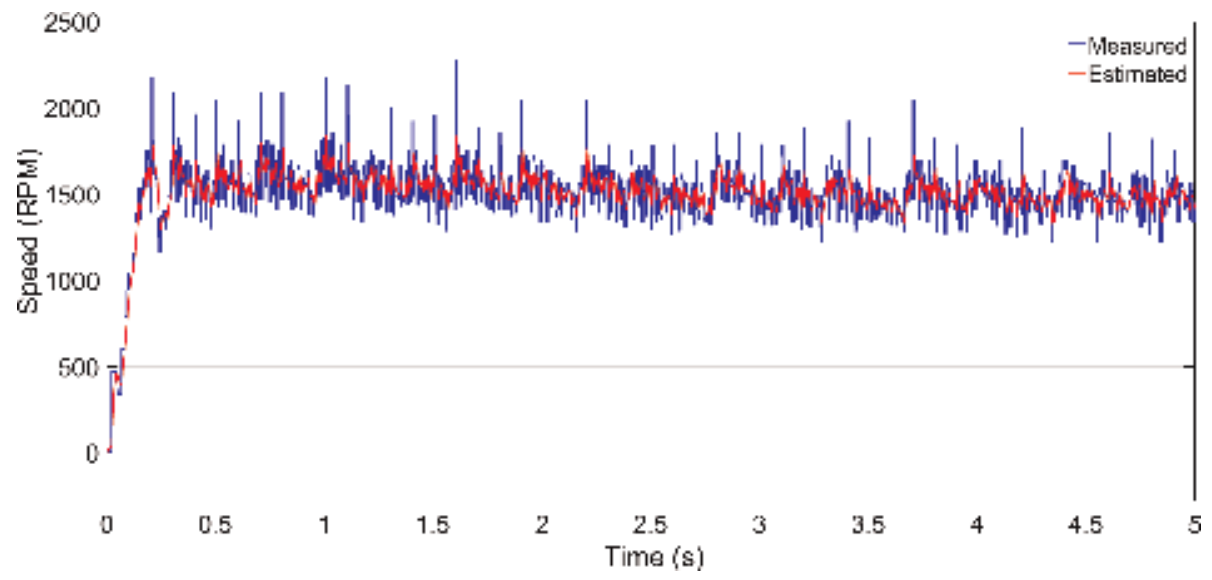

Figure 15.

Speed.

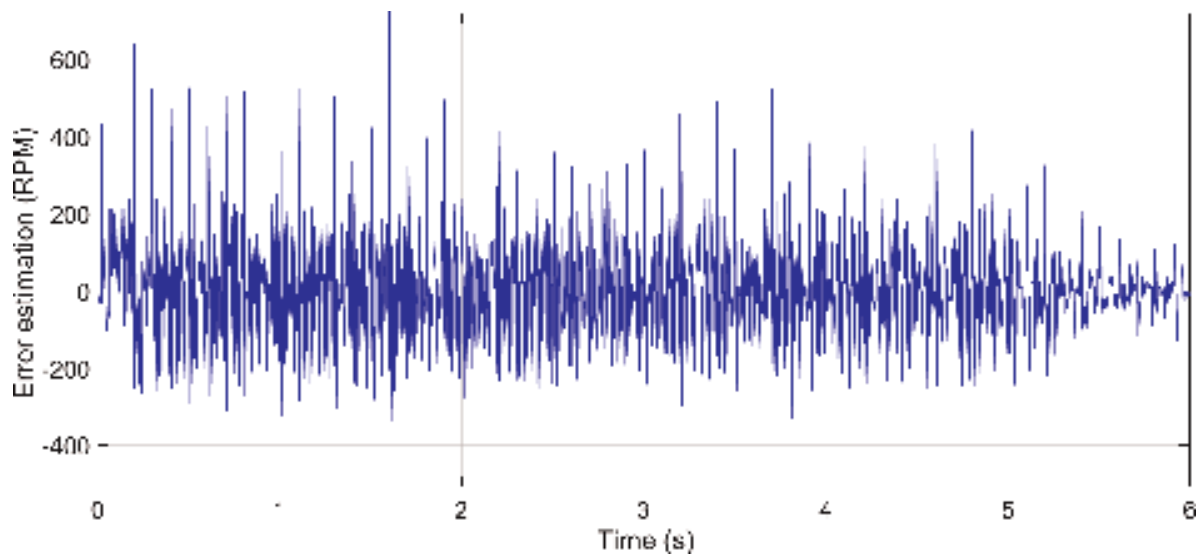

Figure 16.

Error Kalman. 
Figure 15 shows the resulting signal of the controller that stabilizes on the desired speed. The mechanical oscillation is still present, but the controller transient shows to be more smooth than a pure speed controller, allowing a better control of slippery traction situations.

Also Figure 16 proves the use of a Kalman filter instead of a simple state observer for the torque estimation. The figure shows the noise removed from the speed reading without generating an offset of this signal, resulting in a more stable controller.

\section{Conclusion}

This work presents a hardware-in-the-loop system as a solution for fast prototyping, tuning, observation of results, and coding. The process of design by the use of Simulink block diagrams and then automatically generating a code results in a much easier and faster way of designing a code for a complex controller, once the process of coding human error is completely eliminated. Also the possibility of adjusting variables in real time turns out to be a good tool for minor tuning of the controllers, when differences between the real system differs from the mathematical model. Also this work presents a torque controller for a traction motor to solve the slip problem created by high responsible speed controllers, and finally, this work concludes that a torque-generated speed controller shows to be more reliable than both the previous solutions, once the designer has more control over the traction of the vehicle without losing the control of the speed of the vehicle.

\section{Acknowledgements}

This study was financed in part by the Coordenação de Aperfeiçoamento de Pessoal de Nível Superior-Brazil (CAPES)_Finance Code 001.

\section{Author details}

Alex Archela, Dario Guilherme Toginho and Leonimer Flávio de Melo*

State University of Londrina (UEL), Londrina, PR, Brazil

*Address all correspondence to: leonimer@uel.br

\section{IntechOpen}

(C) 2019 The Author(s). Licensee IntechOpen. This chapter is distributed under the terms of the Creative Commons Attribution License (http://creativecommons.org/licenses/ by/3.0), which permits unrestricted use, distribution, and reproduction in any medium, provided the original work is properly cited. (cc) BY 


\section{References}

[1] Yin D, Hori Y. A new approach to traction control of EV based on maximum effective torque estimation. In: 34th Annual Conference of IEEE Industrial Electronics; 2008

[2] Yin D, Hori Y. Traction control for EV based on maximum transmissible torque estimation. International Journal of Intelligent Transportation Systems Research. 2010;8(1):1-9

[3] Magallan GA, De Angelo CH, Garcia GO. Maximization of the traction forces in a 2WD electric vehicle. IEEE Transactions on Vehicular Technology. 2011;60(2):369-380

[4] Heikkinen J, Serykh EV, Minav T. A control strategy for an autonomous differential drive mobile robot. In: 2017 IEEE II International Conference on Control in Technical Systems (CTS); 2017

[5] Kuntanapreeda S. Traction control of electric vehicles using sliding-mode controller with tractive force observer. International Journal of Vehicular Technology. 2014;2014:829097

[6] Mutoh N. Driving and braking torque distribution methods for frontand rear-wheel-independent drive-type electric vehicles on roads with low friction coefficient. IEEE Transactions on Industrial Electronics. 2012;59(10): 3919-3933

[7] Li B, Goodarzi A, Khajepour A, Chen S, Littkouhi B. An optimal torque distribution control strategy for fourindependent wheel drive electric vehicles. Vehicle System Dynamics. 2015;53(8):1172-1189

[8] Hori Y. Future vehicle driven by electricity and control-research on fourwheel-motored "UOT Electric March II”. IEEE Transactions on Industrial Electronics. 2004;51(5):954-962
[9] Kalman RE. A new approach to linear filtering and prediction problems. Journal of Basic Engineering. 1960; 82(1):35-45

[10] Guazzelli PRU, De Oliveira CMR, De Castro AG, Pereira WCA, De Aguiar ML. Electric vehicle hardwarein-the-loop simulation with differentiator optimised by genetic algorithm. In: 2016 12th IEEE International Conference on Industry Applications (INDUSCON); IEEE; 2016

[11] Araújo JFB, Pedrosa HM, Rodrigues MCBP, Barbosa PG. Realtime "hardware-in-the-loop" simulation of components of an electric vehicle powertrain: Modeling and implementation. In: 2016 12th IEEE International Conference on Industry Applications (INDUSCON); IEEE; 2016

[12] Garrido S, Moreno L. Mobile robot path planning using Voronoi diagram and fast marching. In: Robotics, Automation, and Control in Industrial and Service Settings (IGI-Global); 2015

[13] Archela A, Toginho DG, de Melo LF. Torque control of a DC motor with a state space estimator and Kalman filter for vehicle traction. In: 2018 13th IEEE International Conference on Industry Applications (INDUSCON); São Paulo, Brazil; 2018. pp. 763-769. DOI: 10.1109/ INDUSCON.2018.8627285

[14] Philips CL, Nagle HT. Digital Control System Analysis and Design. 4th ed. Upper Saddle River, NJ, USA: Prentice Hall Press; 2007. ISBN: 0130812226, 9780130812223 


\title{
2D-Layered Nanomaterials for Energy Harvesting and Sensing Applications
}

\author{
Po-Kang Yang and Chuan-Pei Lee
}

\begin{abstract}
Nanoscale electromechanical and energy harvesting devices based on few-layer and monolayer two-dimensional (2D) materials with non-symmetric configuration have received enormous attention in recent years. Specifically, piezoelectric and triboelectric devices based on 2D materials for energy harvesting, physical/ chemical sensing, healthcare, and optoelectronics applications have been a growing interest. In this chapter, the typical preparation methods of 2D-layered materials, such as exfoliation methods and chemical vapor phase deposition (CVD), will be discussed first. Then, various characterization techniques by atomic microscopic analysis for 2D materials will be provided briefly. Finally, future aspects of developing 2D piezoelectric and triboelectric devices and their potential applications will be introduced.
\end{abstract}

Keywords: electromechanical, energy harvesting, piezoelectricity, sensors, triboelectricity, two dimensional materials

\section{Introduction}

2D materials have been creating a renaissance in many scientific areas since the adventure of graphene in 2014 [1]. Recently, the family of 2D-layered materials has been profoundly growing up, including transition metal dichalcogenide (TMD) $[2,3]$, transition metal carbide (TMC) [4], and graphene-based materials [5, 6], as shown in Figure 1 [7]. They cover the whole range of material properties from insulators, semiconductors, metals to superconductors, offering a broad portfolio of material's solutions with extraordinary chemical and physical properties for wide applications in promising energy and sensing application technologies [8-11].

For example, piezoelectricity, which is a unique material characteristic, allows effective conversion of ambient mechanical energy into electricity or vice versa. Previously, various TMDs have been applied to fabricate piezoelectric devices owing to their non-centrosymmetric, large piezoelectric coefficients, and layered crystal structure [12]. Prof. Alyörük and his co-workers theoretically predict the piezoelectric constants in various kinds of TMDs [13]. Moreover, Professor Wu's group first demonstrated that a monolayer molybdenum disulfide $\left(\mathrm{MoS}_{2}\right)$ is capable of generating piezoelectric response and piezotronics applications [14]. Meanwhile, Professor Kim's group also reported that bilayer tungsten diselenide $\left(\mathrm{WSe}_{2}\right)$ is a suitable candidate for next generation piezoelectric nanogenerators (PENG) [15]. More importantly, Prof. Li's group have measured the multi-directional piezoelectricity of 


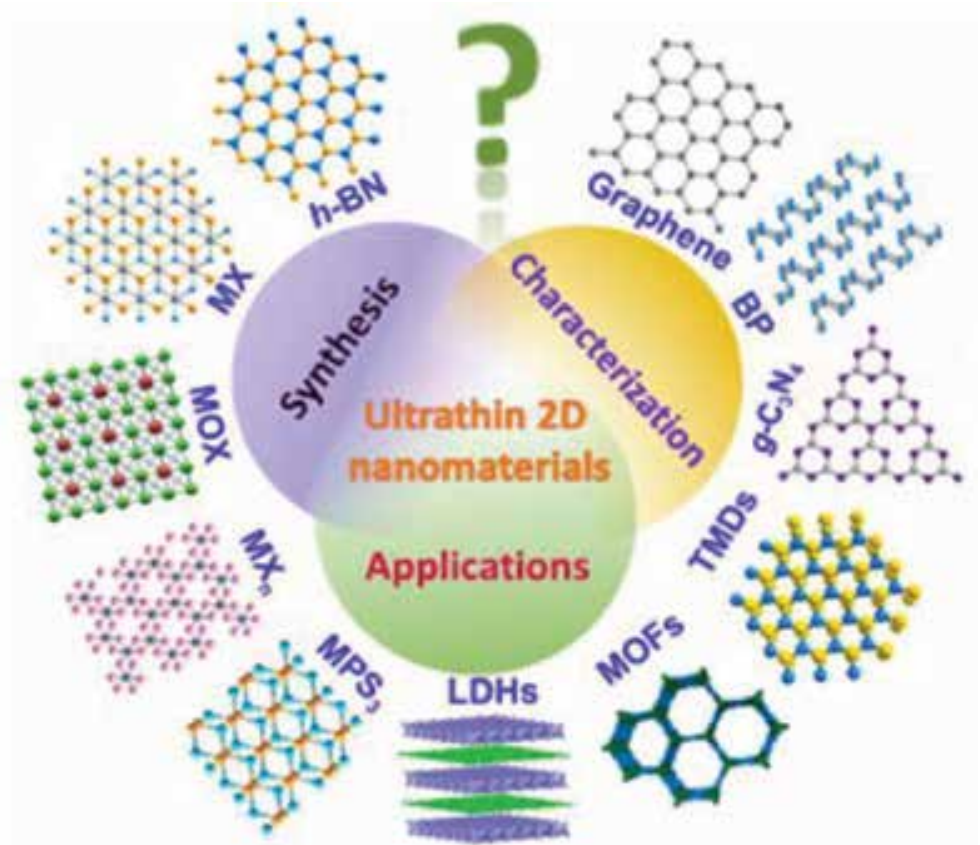

Figure 1.

The schematic assortments of synthetic methods, characterization techniques, and potential device applications of $2 D$ materials [7].
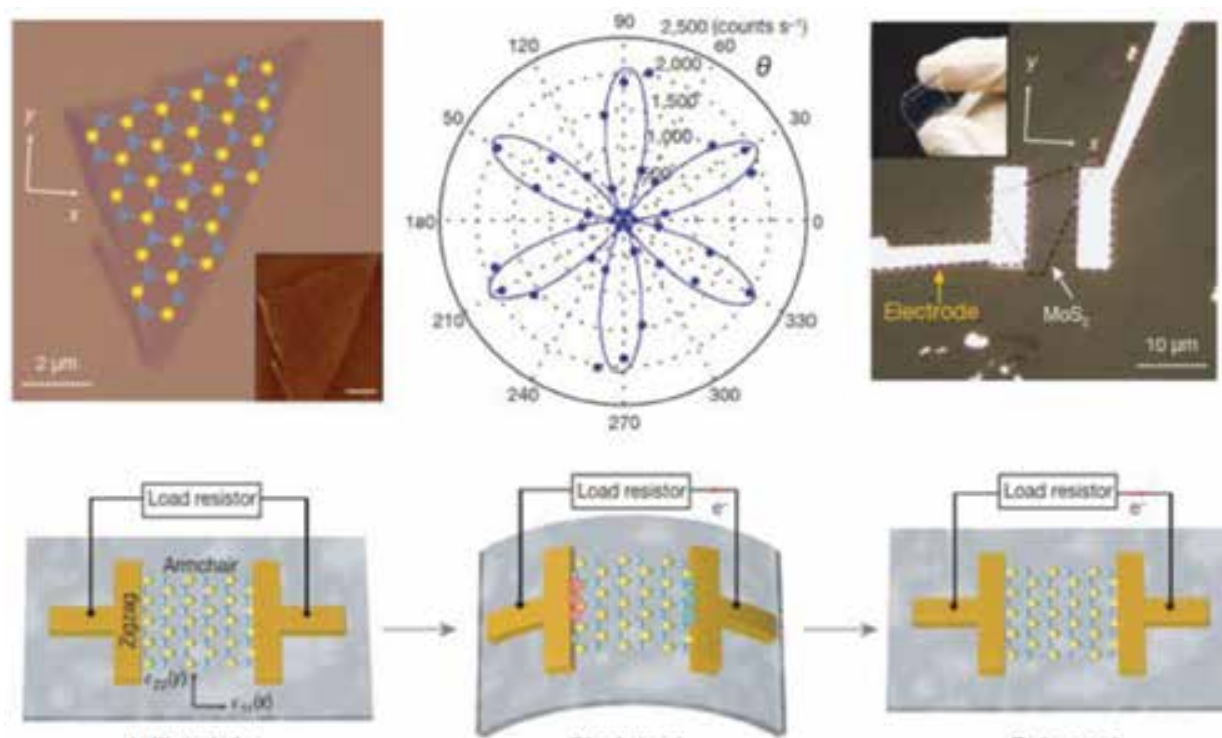

Initial state

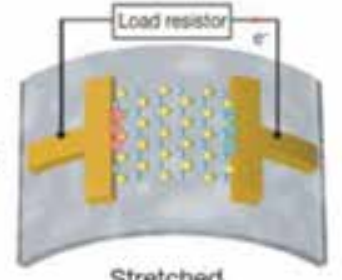

Stretched
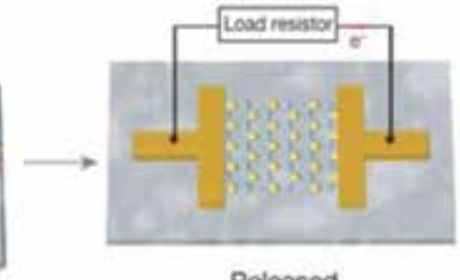

Released

Figure 2.

Schematic of a monolayer $\mathrm{MoS}_{2}$ piezoelectric device and operation scheme [14].

indium selenide $\left(\mathrm{In}_{2} \mathrm{Se}_{3}\right)$ and demonstrated its potential as a biomechanical energy harvesting device for PENG applications [16]. As shown in Figure 2, a 2D materialbased PENG is consisted of a monolayer $\mathrm{MoS}_{2}$ with the multi-layer metal electrode $\mathrm{Cr} / \mathrm{Pd} / \mathrm{Au}$.

Briefly, the basic sequence of the working principle of the as-fabricated PENG is shown in Figure 2. Three stages are involved in charge generation process by 
external mechanical stress, which are initial, stretched, and released states. First, the mechanical strain is applied to the few-layer $\mathrm{MoS}_{2}$ on the PET substrate for different bending radius. Then, the as-fabricated piezoelectric device is coupled to an external load resistor, forming a total electric circuit loop to investigate piezoelectric response. In addition, several attempts of utilizing 2D materials in piezoelectric devices have been found in previous sensor network, including strain sensors [17], pressure sensors [18], and gas sensors [19]. Nevertheless, some challenges may still remain for $2 \mathrm{D}$ material-based piezoelectric devices, including material selection, device reliability, and low electric output power.

Recently, the rise of triboelectric nanogenerators (TENGs) starts a new route to generating electricity from ambient mechanical energy. The TENGs are operated at the basis of well-known contact electrification effect, which were first invented and explained in 2012 (Figure 3; [20]). Within the contact electrification effect, two different materials become mutually charged after it comes into contact with each other. Two electrically charged material causes an electrostatic potential difference, driving the induced electrons to flow via outer circuit loop to provide electricity.

Toward the development of TENGs, 2D materials also become one of the key features. For instance, Prof. Wang's group successfully introduced a monolayer $\mathrm{MoS}_{2}$ as an electron acceptor layer to capture triboelectric charges that result in

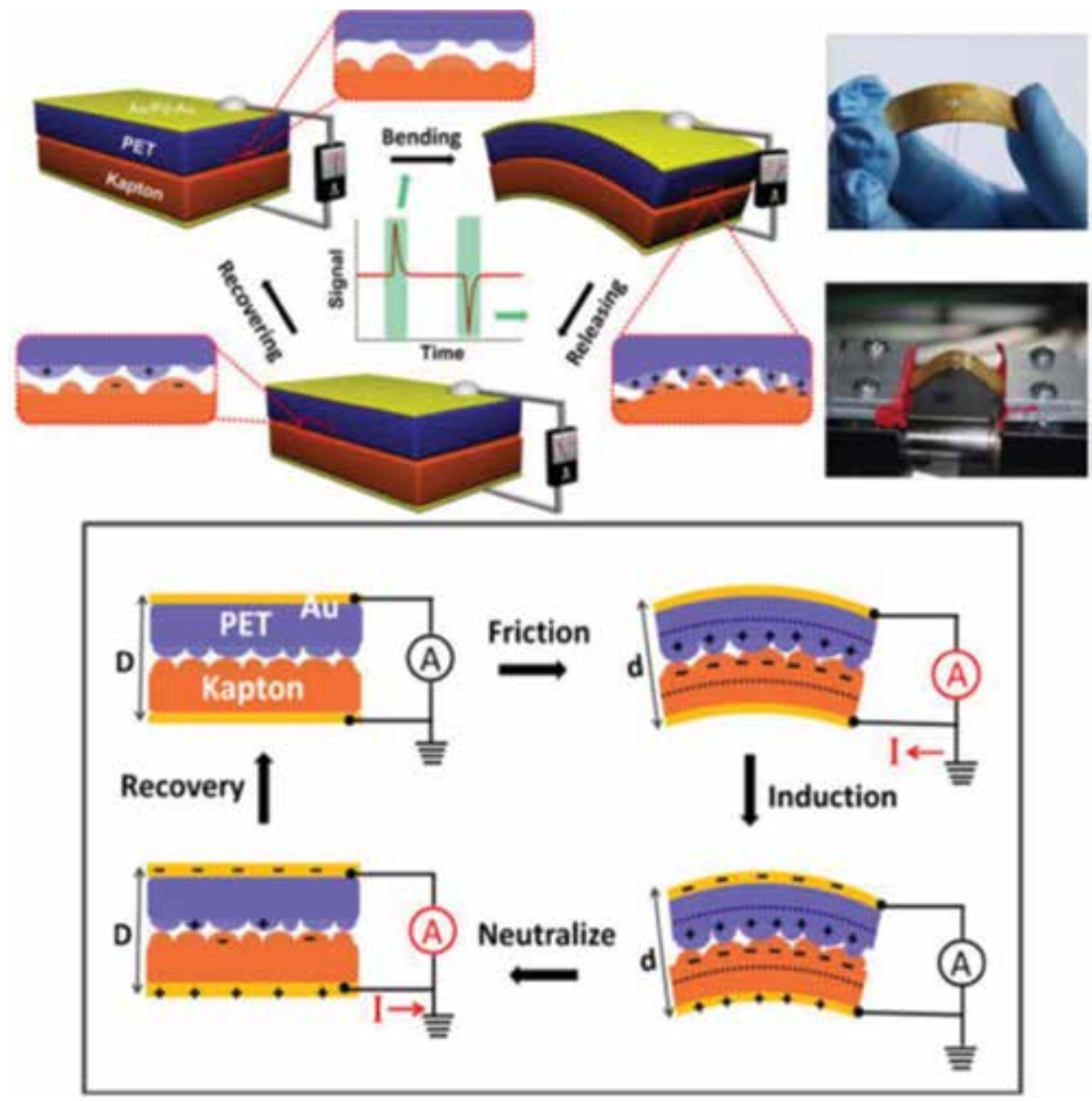

Figure 3.

First prototype triboelectric nanogenerator (TENG) [20]. 

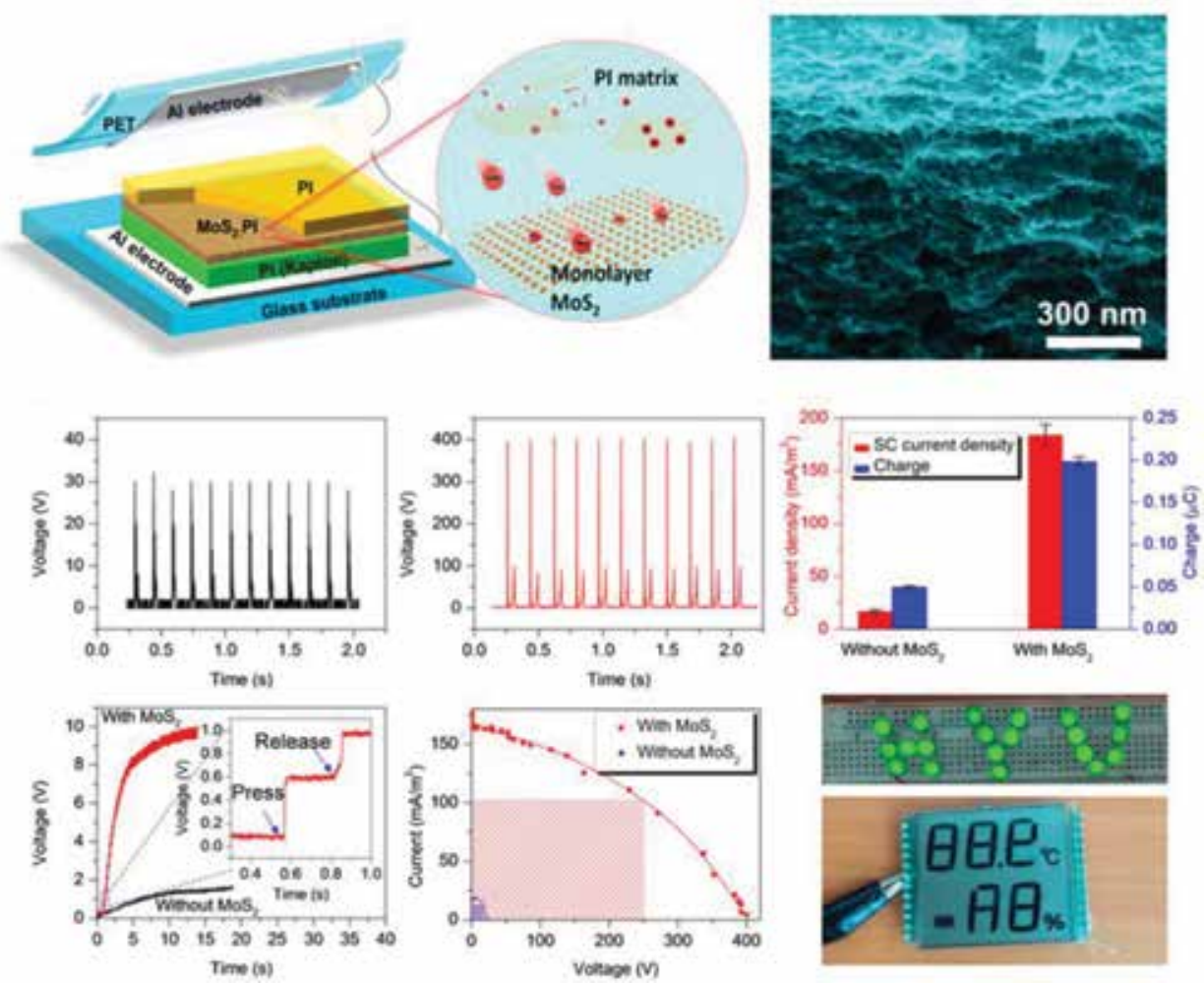

Figure 4 .

A contact-separation mode TENG with monolayer $\mathrm{MoS}_{2}$ [21].
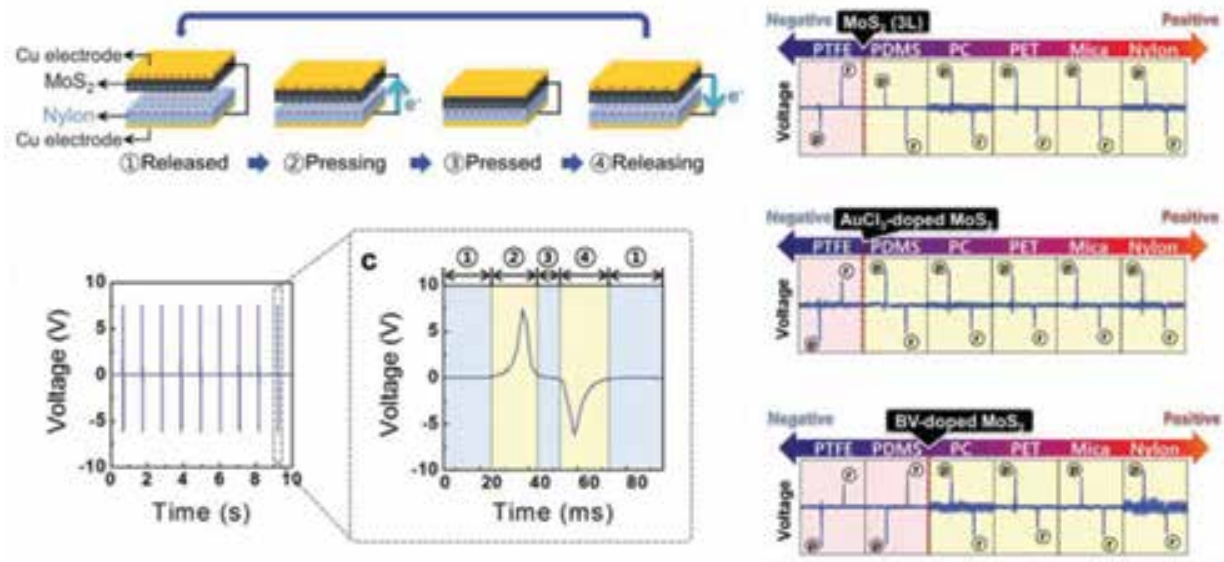

Figure 5.

Triboelectric properties of different $2 D$ materials and their output characteristics [22].

significant output enhancement (Figure 4; [21]). In addition, Prof. Kim's group studied the triboelectric series with various promising 2D materials paving the way for future design rule of 2D material-based TENGs (Figure 5; [22]).

In this chapter, the electromechanical property of 2D materials and their subsequent applications in energy harvesting and sensing fields will be addressed adequately. To begin with, a variety of fabrication methods to prepare 2D materials are briefly described. Sequentially, the exciting progresses of these materials made in both energy harvesting and sensing applications, especially for piezoelectricity, 
triboelectricity, and multi-functional sensing designs, are explored and discussed. Furthermore, future prospects and further developments in above-mentioned research fields based on 2D materials are also commented.

\section{Discussion}

\subsection{Preparation of 2D materials: exfoliation methods}

Traditional approaches to extract single- and few-layer-thick 2D materials from their bulk solids are based on exfoliation methods, which can be categorized into mechanical exfoliation and chemical exfoliation [23]. For mechanical exfoliation (ME) process, it has been widely adopted in preparing diverse $2 \mathrm{D}$ materials, such as graphene [24], phosphorene [25] (Figure 6), and borophenes [26]. The ME process contains several advantages, making them promising for small-scale devices and fundamental researches. For instance, layed Materials prepared from ME process are commonly crystalline and the preparation process is usually rapid. However, the disadvantages of ME method are also obvious, limiting its large-scale production and future applications, such as low material yield, area uniformity, and layer-to-layer asymmetry. As one would like to obtain a certain 2D material with only a few layer or even monolayer, the ME process is relatively time-consuming and inefficient.

\subsection{Preparation of 2D materials: chemical vapor deposition (CVD)}

In contrast to the exfoliation methods, chemical vapor deposition method (CVD) has been profoundly investigated to produce 2D-layered thin film on desirable

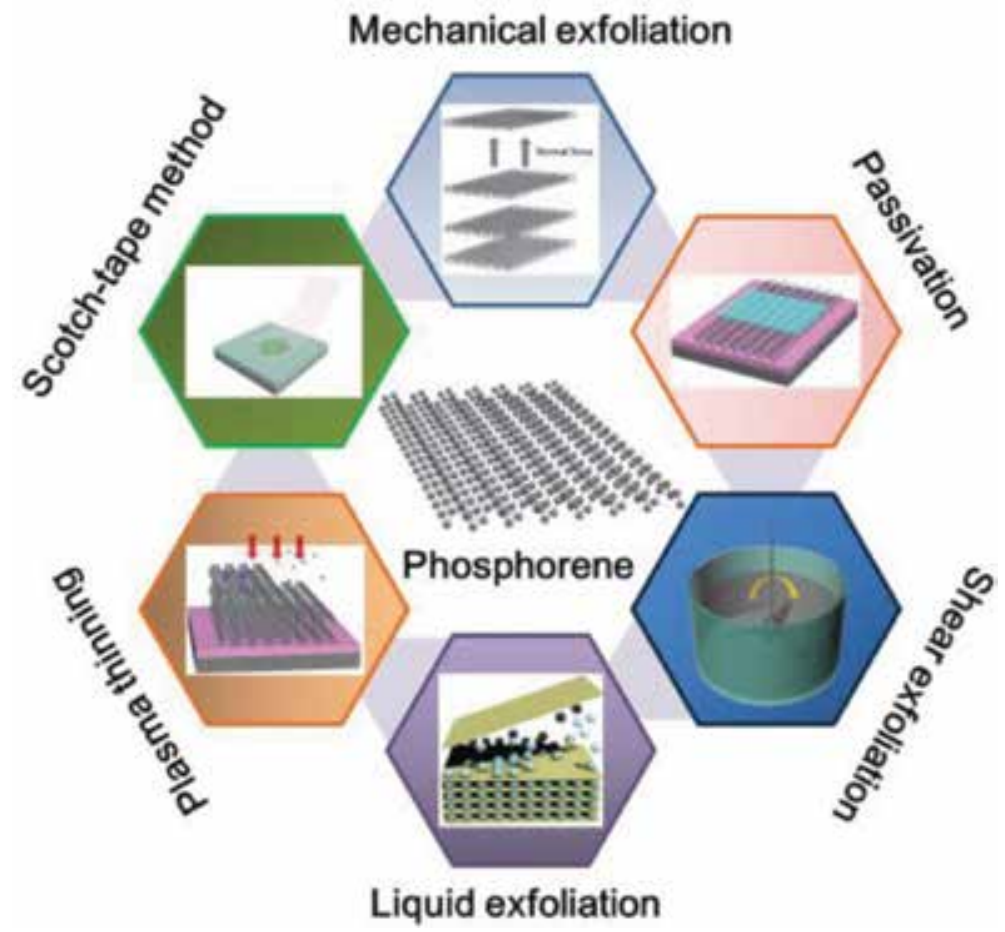

Figure 6.

Schematic representation for the evolution and overview on the Phosphorene fabrication process [25]. 
substrates via the chemical reaction of volatile precursors in recent years [27]. 2D materials grown by CVD methods have been demonstrated to obtain scalable size, controllable thickness, and high crystallinity. Take the tin disulfide $\left(\mathrm{SnS}_{2}\right)$ as an example, according to Yang et al. [28], $\mathrm{SnS}_{2}$ nanosheets could be synthesized on $\mathrm{SiO}_{2}$ substrates by CVD method using $\mathrm{Sn}$ and sulfur as the precursors. The as-synthesized $\mathrm{SnS}_{2}$ flakes could be ranged from 50 to $70 \mu \mathrm{m}$ in lateral dimensions. This synthesis method can produce ultrathin and highly crystalline $\mathrm{SnS}_{2}$ flakes. Meanwhile, it is noted that the $2 \mathrm{D}$ materials with heterostructure can also be produced by multiple CVD process. Revannath et al. presented a p- $\mathrm{MoS}_{2} / \mathrm{n}-\mathrm{MoS}_{2}$ vertical heterostructure by a multiple step CVD process, where the molybdenum oxide $\left(\mathrm{MoO}_{3}\right)$ and sulfur were served as precursors. The as-fabricated heterostructure can be further employed for future light-emitting diode (LED) applications [29]. As discussed above, one can found that 2D materials produced by CVD techniques possess several advantages, such as good quality, high yield, and uniform dimensions. Moreover, 2D materialbased heterostructures can also be obtained by multiple CVD process, which is crucial to both research and industrial applications [30] (Figure 7).

\subsection{Piezoelectricity in 2D materials}

Piezoelectricity was first discovered in 1880, which is due to the accumulated electric charge polarization of materials in response to applied mechanical stress. Previously, bulk materials have been reported to possess the piezoelectric effect, including crystals and polymers. Accordingly, in 2D materials, piezoelectricity is usually attributed to the non-symmetric structure to generate polarization charges in response to the externally applied mechanical stimuli [31]. Recently, owing to the

\section{D materials: from fundamental research to device applications}

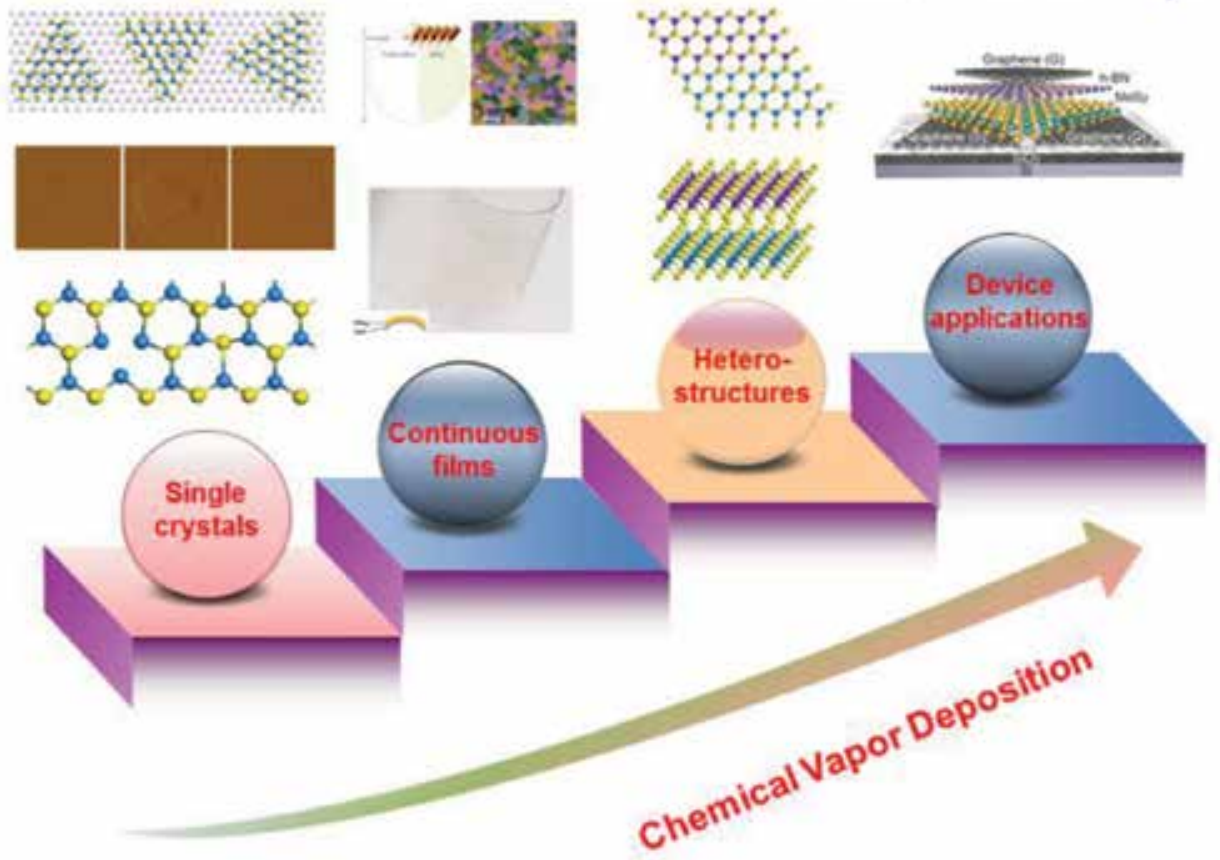

Figure 7.

Roadmap of $2 D$ materials by CVD techniques, from single crystals to device applications [30]. 
continuous growth of wearable, flexible, healthcare, and artificial intelligent robots industry, the market demands for nanoscale and multi-functional sensing devices have become critical, especially for human-machine interface interaction and remote healthcare monitoring. Under these circumstances, $2 \mathrm{D}$ piezoelectric materials with their ultrathin geometry, excellent electromechanical response, and other unique physical properties are suitable candidates and of great importance. Moreover, to directly observe piezoelectricity inside 2D materials, piezoresponse force microscopy (PFM) method is widely implemented, which is based on the converse piezoelectric effect [32, 33] (Figure 8).

\subsection{Piezoelectric devices based on 2D materials}

As mentioned above, after successful inspection of piezoelectricity in 2D materials, a series of correlated applications, including field-effect transistors (FET), sensors, catalytic reactions, optoelectronics, and energy storage, are emerged [34-38]. Herein, we will briefly review the device application of 2D piezoelectric materials, especially for energy harvesting device development. For example, in 2017, Muralidharan et al. present a mechano-electrochemical device configuration based on sodiated black phosphorus (BP) nanosheets, where this device is capable of harvesting low frequency mechanical energy at $0.01 \mathrm{~Hz}$ [39] (Figure 9).

In addition, Lee et al. have also developed a monolayer $\mathrm{WSe}_{2}$ piezoelectric nanogenerator (PENG), which can provide an output voltage of $45 \mathrm{mV}$ (Figure 9). The output electrical signal will only appear during the moment of stretching and releasing from external strain. This proves the concept of using this device to generate electricity from mechanical stimuli. Moreover, one can also see that the output voltage and current increase with increasing tensile strain. Furthermore, the as-fabricated PENG can sustain stable electrical output even after 3 hours, demonstrating its excellent device reliability.
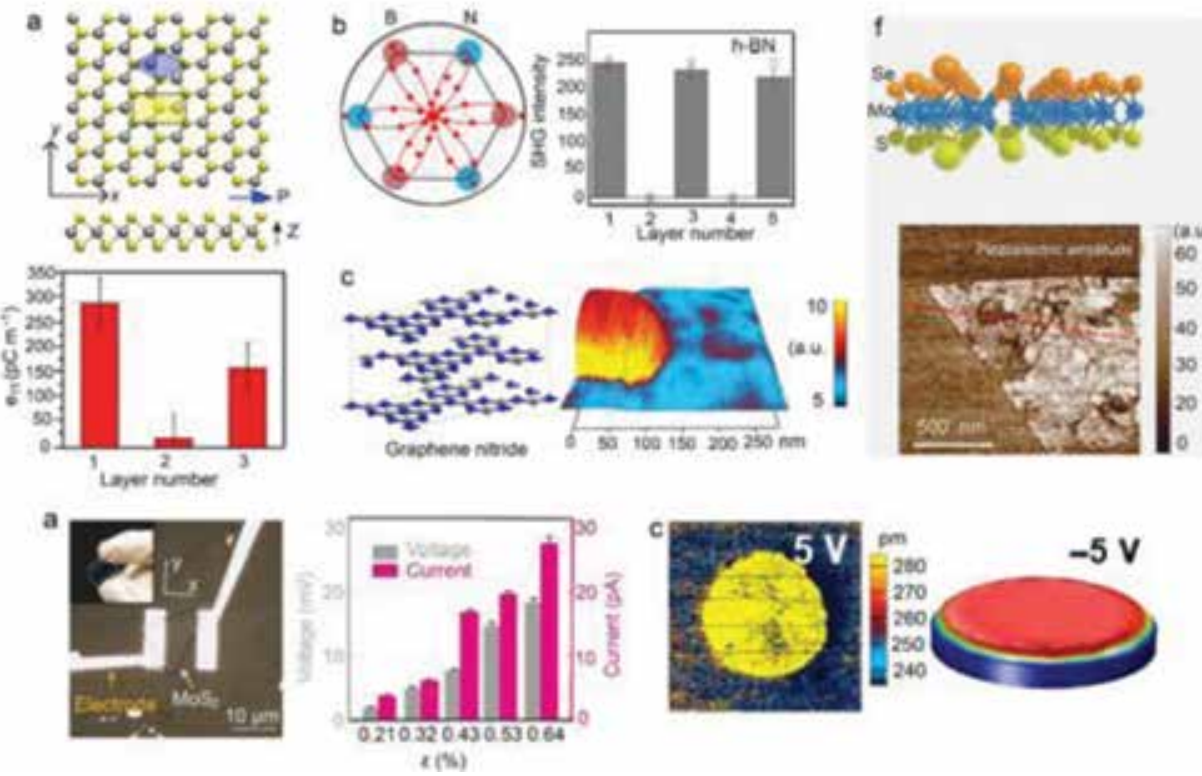

Figure 8.

Piezoelectricity observation in various $2 \mathrm{D}$ materials, including $\mathrm{MoS}_{2}$, boron nitride, graphene nitride, and monolayer MoSSe [32, 33]. 

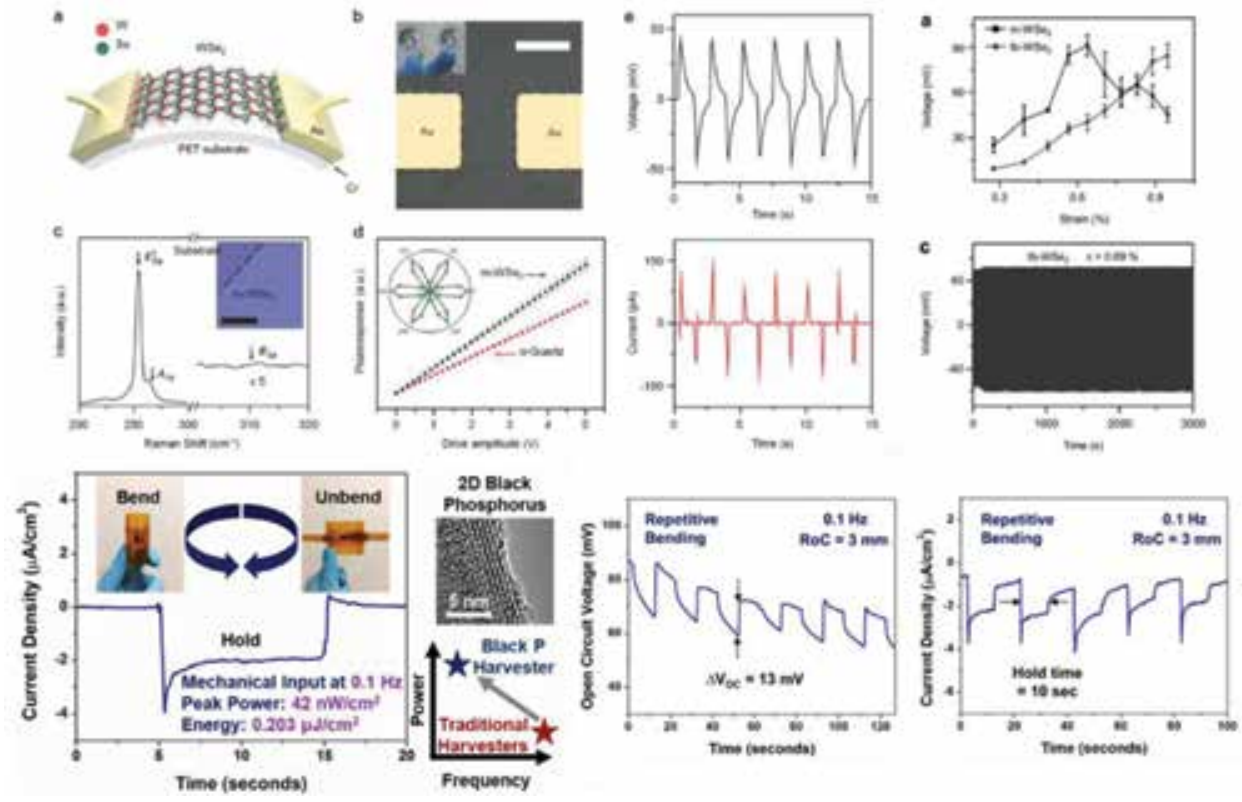

Figure 9.

Piezoelectric nanogenerator based on $2 D$ materials, where $W \mathrm{We}_{2}$ and black phosphorus are shown as examples [39].
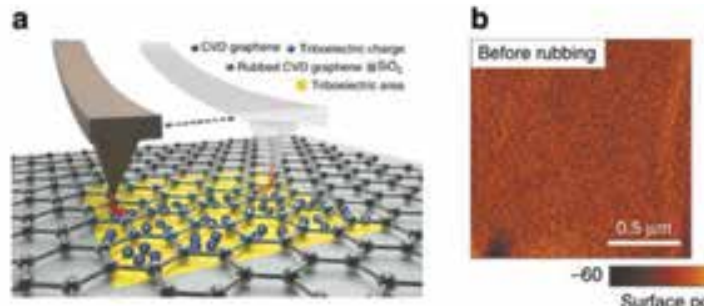

c

-60 Suctace potentiat (min)
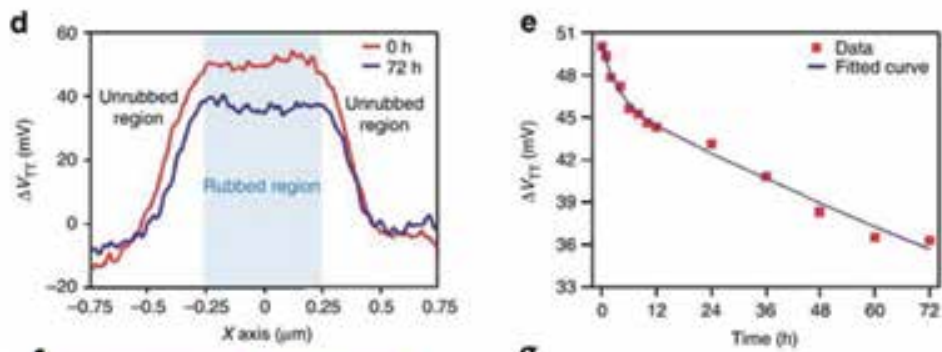

f
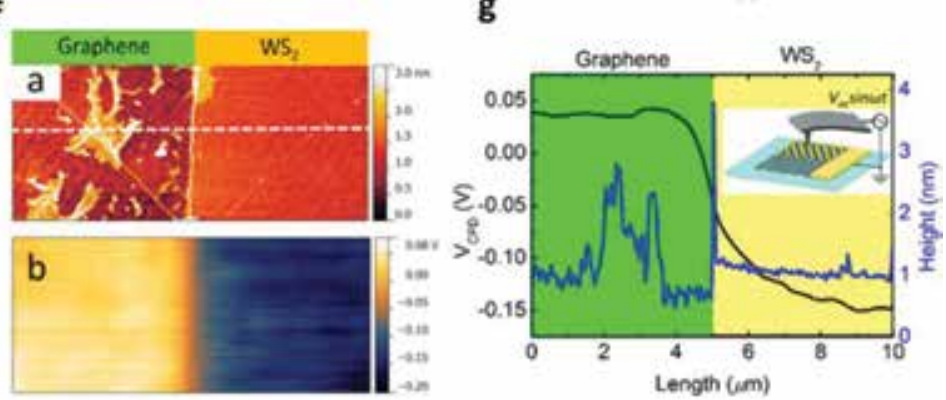

Figure 10.

Observing triboelectrification by friction of graphene with a Pt AFM tip (a-e). Schematic diagram for measuring the triboelectricity of the subpart labels of $a$ and $b$ in $(f)$ show the topographic image of a grapheneWS2 heterojunction and corresponded surface potential measured by KPFM, respectively $(f, g)$ [41-43]. 


\subsection{Triboelectricity in 2D materials}

Triboelectric charging is a well-known electrical charging phenomenon of materials, which has been studied for more than 2500 years [40]. The triboelectric charging phenomenon occurs at two different materials, which come into contact and separate with each other. Owing to the charge transfer during contact, charges of opposite signs accumulate on the surface of each material, thereby developing static electricity or so-called triboelectricity. In addition to conventional thin-film and bulk materials, recently, 2D materials, such as TMDs and graphene (GR), have also been found to exhibit considerable triboelectricity [41-43]. Generally, Kelvin probe force microscope (KPFM) and Scanning Kelvin Probe microscopy (SKPM) have been utilized into characterizing surface potential and surface work function. The KPFM method allows one to measure and compare the surface potentials of the dielectrics before and after friction, which is highly correlated to the triboelectricity (Figure 10).
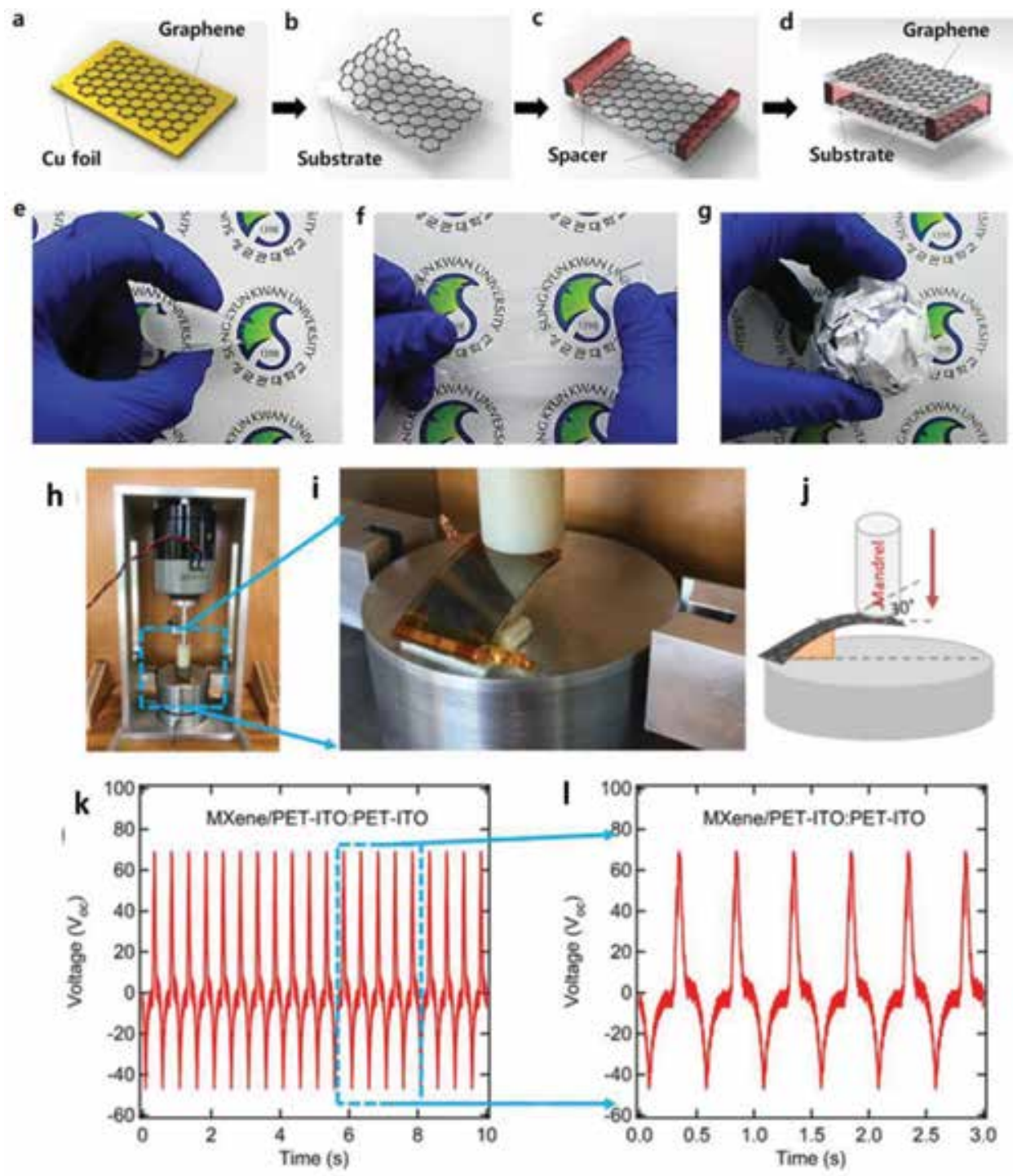

Figure 11.

Schematic diagrams of device fabrication and compatibility of graphene with an arbitrary substrate $(a-g)$. The flexible MXene TENG was operated with a force of $1 \mathrm{~N}$ applied at $2 \mathrm{~Hz}$ by the mandrel $(h-l)[44,45]$. 


\subsection{Triboelectric devices based on 2D materials}

To further understand and evaluate the triboelectricity inside 2D materials, several attempts have been made to fabricate triboelectric devices based on 2D materials. For instance, Kim et al. reported the first flexible, transparent TENG device using graphene [44]. The as-fabricated TENGs were able to power commercial LEDs by using the electrical power output generated from TENG without any other external energy source. Meanwhile, Dong et al. reported high-performance TENG device by new materials, such as fluorinated MXene, and successfully demonstrated to both rigid and flexible TENGs applications. Furthermore, these MXene-based TENGs can be further integrated into accessories, wrist bands, and textiles [45] (Figure 11).

\section{Conclusion}

For 2D materials, future challenges and aspects for fundamental research and industrial applications, especially for energy harvesting and sensing field, may be summarized as follows. Though various kinds of 2D materials have been explored to possess either piezoelectricity or triboelectricity, most of them are not well investigated owing to the difficult material preparation process via exfoliation or CVD method. To be more specific, several key factors still need to be further understood in both 2D piezoelectric and triboelectric materials. In 2D piezoelectric materials, first, it has been well known that the band structure could be affected by piezoelectric field created by strain; therefore, the influence of strain on band structure of 2D materials should be thoroughly investigated. Second, optimize synthetic methods of 2D piezoelectric materials are required, playing a key role for further improving output characteristics of piezoelectric devices. Meanwhile, as for 2D triboelectric materials, compared with traditional triboelectric materials, they have shown great advantages, such as ultrathin film feasibility, flexibility, and process compatibility with large array devices, advancing its applications as future wearable sensors and human-machine bridging interface. Nevertheless, further efforts still need to be made in the following aspects. First, an entirely qualitative and quantitative characterization of 2D triboelectricity should be implemented to understand the triboelectric charge transferring process. Moreover, 2D triboelectric materials in a large scale with low costs, high uniformity, and low-temperature synthesis process should be achieved, which will be beneficial for next-generation flexible device developments.

\section{Acknowledgements}

This work was supported by the Ministry of Science and Technology (MOST) of Taiwan. Professor Chuan-Pei Lee especially thanks the financial support of MOST, under grant numbers 107-2113-M-845-001-MY3).

\section{Conflict of interest}

The authors declare no competing financial interests. 


\section{Author details}

Po-Kang Yang ${ }^{1}$ and Chuan-Pei Lee ${ }^{2 *}$

1 Graduate Institute of Nanomedicine and Medical Engineering, College of Biomedical Engineering, Taipei Medical University, Taiwan

2 Department of Applied Physics and Chemistry, University of Taipei, Taiwan

*Address all correspondence to: cplee@utaipei.edu.tw

\section{IntechOpen}

(C) 2019 The Author(s). Licensee IntechOpen. This chapter is distributed under the terms of the Creative Commons Attribution License (http://creativecommons.org/licenses/ by/3.0), which permits unrestricted use, distribution, and reproduction in any medium, provided the original work is properly cited. (cc) BY 


\section{References}

[1] Paton KR, Varrla E, Backes C, Smith RJ, Khan U, O’Neill A, et al. Scalable production of large quantities of defect-free few-layer graphene by shear exfoliation in liquids. Nature Materials. 2014;13:624

[2] Butler SZ, Hollen SM, Cao L, Cui Y, Gupta JA, Gutiérrez HR, et al. Progress, challenges, and opportunities in two-dimensional materials beyond graphene. ACS Nano. 2013;7:2898

[3] Pham VP, Yeom GY. Recent advances in doping of molybdenum disulfide industrial applications and future prospects. Advanced Materials. 2016;28:9024

[4] Shahzad F, Alhabeb M, Hatter CB, Anasori B, Man Hong S, Koo CM, et al. Electromagnetic interference shielding with 2D transition metal carbides (MXenes). Science. 2016;353:1137

[5] Chen P-Y, Liu M, Valentin TM, Wang Z, Spitz Steinberg R, Sodhi $J$, et al. Hierarchical metal oxide topographies replicated from highly textured graphene oxide by intercalation templating. ACS Nano. 2016;10:10869

[6] Pham VP, Jang HS, Whang D, Choi JY. Direct growth of graphene on rigid and flexible substrates: Progress, applications, and challenges. Chemical Society Reviews. 2017;46:6276

[7] Tan C, Cao X, Wu X-J, He Q, Yang J, Zhang $X$, et al. Recent advances in ultrathin two-dimensional nanomaterials. Chemical Reviews. 2017;117:6225

[8] Lee C-P, Lin C-A, Wei T-C, Tsai M-L, Meng Y, Li C-T, et al. A paper-based electrode using a graphene dot/PEDOT: PSS composite for flexible solar cells. Nano Energy. 2015;18:109
[9] Yang PK, Chang W-Y, Teng P-Y, Jeng S-F, Lin S-J, Chiu P-W, et al. Fully transparent resistive memory employing graphene electrodes for eliminating undesired surface effects. Proceedings of the IEEE. 2013;101:1732

[10] Chen K-S, Balla I, Luu NS, Hersam MC. Emerging opportunities for two-dimensional materials in lithiumion batteries. ACS Energy Letters. 2017;2:2026

[11] Mao S, Chang J, Pu H, Lu G, He $\mathrm{Q}$, Zhang H, et al. Two-dimensional nanomaterial-based field-effect transistors for chemical and biological sensing. Chemical Society Reviews. 2017;46:6872

[12] Li H, Shi Y, Chiu M-H, Li L-J. Emerging energy applications of two-dimensional layered transition metal dichalcogenides. Nano Energy. 2015;18:293

[13] Alyörük MM, Aierken Y, Çakır D, Peeters FM, Sevik C. Promising piezoelectric performance of single layer transition-metal dichalcogenides and dioxides. Journal of Physical Chemistry C. 2015;119:23231

[14] Wu WZ, Wang L, Li YL, Zhang F, Lin L, Niu SM, et al. Piezoelectricity of single-atomic-layer $\mathrm{MoS}_{2}$ for energy conversion and piezotronics. Nature. 2014;514:470

[15] Lee JH, Park JY, Cho EB, Kim TY, Han SA, Kim TH, et al. Reliable piezoelectricity in bilayer $\mathrm{WSe}_{2}$ for piezoelectric nanogenerators. Advanced Materials. 2017;29:1606667

[16] Xue F, Zhang J, Hu W, Hsu W-T, Han A, Leung S-F, et al. Multidirection piezoelectricity in mono-and multilayered hexagonal $\alpha-\mathrm{In}_{2} \mathrm{Se}_{3}$. ACS Nano. 2018;12:4976 
[17] Qi J, Lan YW, Stieg AZ, Chen JH, Zhong YL, Li LJ, et al. Piezoelectric effect in chemical vapour depositiongrown atomic-monolayer triangular molybdenum disulfide piezotronics. Nature Communications. 2015;6:7430

[18] Park M, Park YJ, Chen X, Park Y-K, Kim M-S, Ahn J-H. $\mathrm{MoS}_{2}$-based tactile sensor for electronic skin applications. Advanced Materials. 2016;28:2556

[19] He QY, Zeng ZY, Yin ZY, Li H, Wu SX, Huang X, et al. Fabrication of flexible $\mathrm{MoS}_{2}$ thin-film transistor arrays for practical gas-sensing applications. Small. 2012;8:2994

[20] Fan FR, Tian ZQ, Wang ZL. Flexible triboelectric generator. Nano Energy. 2012;328:328

[21] Wu C, Kim TW, Park JH, An H, Shao J, Chen X, et al. Nhanced triboelectric nanogenerators based on $\mathrm{MoS}_{2}$ monolayer nanocomposites acting as electronacceptor layers. ACS Nano. 2017;11:8356

[22] Seol M, Kim S, Cho Y, Byun K-E, Kim H, Kim J, et al. Triboelectric series of 2D layered materials. Advanced Materials. 2018;30:1801210

[23] Fiori G, Bonaccorso F, Iannaccone G, Palacios T, Neumaier D, Seabaugh A, et al. Electronics based on twodimensional materials. Nature Nanotechnology. 2014;9:768

[24] Nicolosi V, Chhowalla M, Kanatzidis MG, Strano MS, Coleman JN. Liquid exfoliation of layered materials. Science. 2013;340:1420

[25] Dhanabalan SC, Ponraj JS, Guo Z, Li S, Bao Q, Zhang H. Emerging trends in phosphorene fabrication towards next generation devices. Advancement of Science. 2017;4:1600305

[26] Penev ES, Artyukhov VI, Ding F, Yakobson BI. Polymorphism of two-dimensional boron. Advanced Materials. 2012;24:4956

[27] Chang Y-H, Zhang W, Zhu Y, Han Y, $\mathrm{Pu}$ J, Chang J-K, et al. Tin disulfide; an emerging layered metal dichalcogenide semiconductor: Materials properties and device characteristics. ACS Nano. 2014;8:8582

[28] Yang Y-B, Dash JK, Littlejohn AJ, Xiang Y, Wang Y, Shi J, et al. Large single crystal $\mathrm{SnS}_{2}$ flakes synthesized from coevaporation of Sn and S. Crystal Growth \& Design. 2016;16:961

[29] Nikam RD, Sonawane PA, Sankar $\mathrm{R}$, Chen Y-T. Epitaxial growth of vertically stacked $\mathrm{p}-\mathrm{MoS}_{2} / \mathrm{n}-\mathrm{MoS}_{2}$ heterostructures by chemical vapor deposition for light emitting devices. Nano Energy. 2017;32:454

[30] Cai Z, Liu B, Zou X, Cheng H-M. Chemical vapor deposition growth and applications of two-dimensional materials and their heterostructures. Chemical Reviews. 2018;118:6091

[31] Hinchet R, Khan U, Falconi C, Kim $\mathrm{S}-\mathrm{W}$. Piezoelectric properties in twodimensional materials: Simulations and experiments. Materials Today. 2018;21:611

[32] Gomez A, Gich M, CarreteroGenevrier A, Puig T, Obradors X. Piezogenerated charge mapping revealed through direct piezoelectric force microscopy. Nature Communications. 2017;8:1113

[33] Cui C, Xue F, Hu W-J, Li L-J. Twodimensional materials with piezoelectric and ferroelectric functionalities. npj 2D Materials and Applications. 2018;2:18

[34] Xue Y, Zhang Q, Wang W, Cao H, Yang Q, Fu L. Opening twodimensional materials for energy conversion and storage: A concept. 
Advanced Energy Materials. 2017;7:1602684

[35] Rao CNR, Gopalakrishnan K, Maitra U. Comparative study of potential applications of graphene, $\mathrm{MoS}_{2}$, and other two-dimensional materials in energy devices, sensors, and related areas. ACS Applied Materials \& Interfaces. 2015;7:7809

[36] Varghese SS, Varghese SH, Swaminathan S, Singh KK, Mittal V. Two-dimensional materials for sensing: Graphene and beyond. Electronics. 2015;4:651

[37] Zeng M, Xiao Y, Liu J, Yang K, $\mathrm{Fu}$ L. Exploring two-dimensional materials toward the next-generation circuits: From monomer design to assembly control. Chemical Reviews. 2018;118:6236

[38] Deng DH, Noveselov KS, Fu Q, Zheng NF, Tian ZQ, Bao XH. Catalysis with two-dimensional materials and their heterostructures. Nature Nanotechnology. 2016;11:218

[39] Muralidharan N, Li M, Carter RE, Galioto N, Pint CL. Ultralow frequency electrochemical-mechanical strain energy harvester using 2D black phosphorus nanosheets. ACS Energy Letters. 2017;2:1797

[40] Lowell J, Rose-Innes AC. Contact electrification. Advances in Physics. 1980;29:947

[41] Kim KN, Jung YK, Chun J, Ye BU, Gu M, Seo E, et al. Surface dipole enhanced instantaneous charge pair generation in triboelectric nanogenerator. Nano Energy. 2016;26:360-370

[42] Kim S, Kim TY, Lee KH, Kim TH, Cimini FA, Kim SK, et al. Rewritable ghost floating gates by tunnelling triboelectrification for two-dimensional electronics. Nature Communications. 2017;8:15891
[43] Zheng C, Zhang Q, Weber B, Ilatikhameneh H, Chen F, Sahasrabudhe $\mathrm{H}$, et al. Direct observation of 2D electrostatics and ohmic contacts in template-grown graphene/ $\mathrm{WS}_{2}$ heterostructures. ACS Nano. 2017;11:2785

[44] Kim S, Gupta MK, Lee KY, Sohn A, Kim TY, Shin K-S, et al. Transparent flexible graphene triboelectric nanogenerators. Advanced Materials. 2014;26:3918

[45] Dong Y, Mallineni SSK, Maleski K, Behlow H, Mochalin VN, Rao AM, et al. Metallic MXenes: A new family of materials for flexible triboelectric nanogenerators. Nano Energy.

2018;44:103 



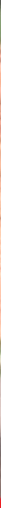

\section{Edited by Adel El-Shahat and Mircea Ruba}

In this book, highly qualified multidisciplinary scientists present their recent research that has been motivated by the significance of applied electromechanical devices and machines for electric mobility solutions. It addresses advanced applications and innovative case studies for electromechanical parameter identification, modeling, and testing of; permanent-magnet synchronous machine drives; investigation on internal short circuit identifications; induction machine simulation; CMOS active inductor applications; low-cost wide-speed operation generators; hybrid electric vehicle fuel consumption; control technologies for high-efficient applications; mechanical and electrical design calculations; torque control of a DC motor with a state-space estimation; and 2D-layered nanomaterials for energy harvesting. This book is essential reading for students, researchers, and professionals interested in applied electromechanical devices and machines for electric mobility solutions.

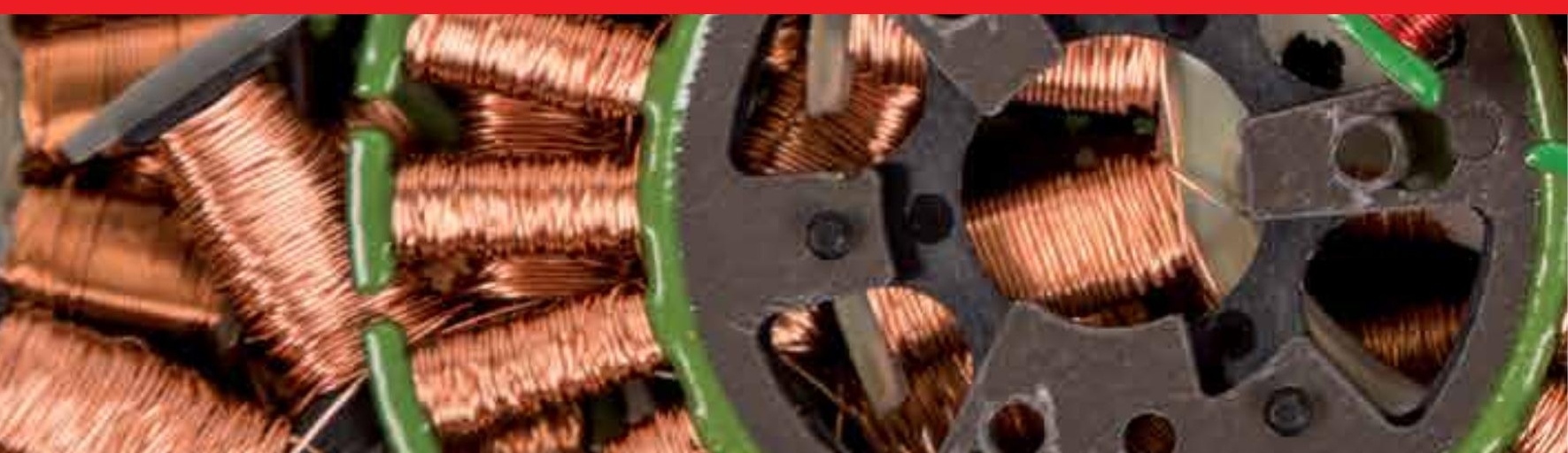

